\title{
The Local Pro-p Anabelian Geometry of Curves
}

\author{
by Shinichi Mochizuki
}

\section{INTRODUCTION}

\section{The Anabelian Geometry of Grothendieck:}

Let $X$ be a connected scheme. Then one can associate (after Grothendieck) to $X$ its algebraic fundamental group $\pi_{1}(X)$. This group $\pi_{1}(X)$ is a profinite group which is uniquely determined (up to inner automorphisms) by the property that the category of finite, discrete sets equipped with a continuous $\pi_{1}(X)$-action is equivalent to the category of finite étale coverings of $X$. Moreover, the assignment $X \mapsto \pi_{1}(X)$ is a functor from the category of connected schemes (and morphisms of schemes) to the category of profinite topological groups and continuous outer homomorphisms (i.e., continuous homomorphisms of topological groups, where we identify any two homomorphisms that can be obtained from one another by composition with an inner automorphism).

Now let $K$ be a field. Let $\Gamma_{K}$ be the absolute Galois group of $K$. Then $\pi_{1}(\operatorname{Spec}(K))$ may be identified with $\Gamma_{K}$. Let $X_{K}$ be a variety (i.e., a geometrically integral separated scheme of finite type) over $K$. Then the structure morphism $X_{K} \rightarrow \operatorname{Spec}(K)$ defines a natural augmentation $\pi_{1}\left(X_{K}\right) \rightarrow \Gamma_{K}$. The kernel of this morphism $\pi_{1}\left(X_{K}\right) \rightarrow \Gamma_{K}$ is a closed normal subgroup of $\pi_{1}\left(X_{K}\right)$ - called the geometric fundamental group of $X_{K}$ which may be identified with $\pi_{1}\left(X_{\bar{K}}\right)$ (where $X_{\bar{K}} \stackrel{\text { def }}{=} X_{K} \otimes_{K} \bar{K}$ ). If, moreover, one fixes a prime number $p$, then one can form the maximal pro-p quotient $\pi_{1}\left(X_{\bar{K}}\right)^{(p)}$ of $\pi_{1}\left(X_{\bar{K}}\right)$. Since the quotient $\pi_{1}\left(X_{\bar{K}}\right) \rightarrow \pi_{1}\left(X_{\bar{K}}\right)^{(p)}$ is characteristic, it follows that the kernel of this quotient is, in fact, a normal subgroup of $\pi_{1}\left(X_{K}\right)$. The quotient of $\pi_{1}\left(X_{K}\right)$ by this normal subgroup will be denoted $\Pi_{X_{K}}$. Thus, $\Pi_{X_{K}}$ inherits a natural augmentation $\Pi_{X_{K}} \rightarrow \Gamma_{K}$ from that of $\pi_{1}\left(X_{K}\right)$.

Now let us consider the assignment

$$
\pi_{1}(-)_{K}:\left\{X_{K} \rightarrow \operatorname{Spec}(K)\right\} \mapsto\left\{\pi_{1}\left(X_{K}\right) \rightarrow \Gamma_{K}\right\}
$$

This assignment defines a functor from the category $\mathcal{C}_{K}$ of $K$-varieties (whose morphisms are $K$-linear morphisms of varieties) to the category $\mathcal{G}_{K}$ whose objects are profinite topological groups equipped with an augmentation to $\Gamma_{K}$, and whose morphisms are continuous outer homomorphisms of topological groups that lie over $\Gamma_{K}$. It was the intuition of Grothendieck (see [Groth]) that: 
For certain types of $K$, if one replaces $\mathcal{C}_{K}$ and $\mathcal{G}_{K}$ by "certain appropriate" subcategories $\mathcal{C}_{K}^{\prime}$ and $\mathcal{G}_{K}^{\prime}$ (such that $\pi_{1}(-)_{K}$ still maps $\mathcal{C}_{K}^{\prime}$ into $\left.\mathcal{G}_{K}^{\prime}\right)$, then $\pi_{1}(-)_{K}$ should be fully faithful.

Here, the "certain appropriate" subcategories $\mathcal{C}_{K}^{\prime}$ for which this piece of intuition was to hold true were tentatively assigned the appellation anabelian, while the piece of intuition itself came to be referred to as Grothendieck's Conjecture of Anabelian Geometry (or, simply, the "Grothendieck Conjecture," for short). Roughly speaking, the sorts of varieties that were thought to be likely to be "anabelian" were varieties that are "sufficiently hyperbolic." (Note that, as one can see in the case of curves, hyperbolic varieties tend to have highly nonabelian fundamental groups, hence the term "anabelian." In higher dimensions, however, things are not so simple - see, e.g., [IN].)

A variant of the above "profinite" Grothendieck Conjecture is the following "pro$p$ " Grothendieck Conjecture: Namely, instead of considering $\pi_{1}(-)_{K}$, one considers the functor that assigns to the $K$-variety $X_{K}$ the augmented group $\Pi_{X_{K}} \rightarrow \Gamma_{K}$. The "pro- $p$ " Grothendieck Conjecture then asserts that, in certain situations, this functor should be fully faithful. The present paper is concerned with proving various versions of the profinite and pro- $p$ Grothendieck Conjectures under various conditions.

\section{Statement of the Main Results:}

Let $p$ be a prime number. Let $K$ be a sub-p-adic field (cf. Definition 15.4 (i)), i.e., a subfield of a finitely generated field extension of $\mathbf{Q}_{p}$. In this paper, we prove a pro-p version of the Grothendieck Conjecture (Theorem A) for dominant morphisms between "smooth pro-varieties" and "hyperbolic pro-curves" over $K$. (Here, by a "smooth pro-variety" (respectively, "hyperbolic pro-curve") over $K$, we mean a $K$-scheme which can be written as a projective limit of smooth varieties (respectively, hyperbolic curves) over $K$ in which the transition morphisms are birational - cf. Definitions 15.4 (ii) and 16.4.) We then give various versions of Theorem $A$ (namely, Theorems $A^{\prime}$ and $A^{\prime \prime}$ ) for truncated fundamental groups. From Theorem $A$, we also derive a profinite version of the Grothendieck Conjecture (Theorem $B$ ) for morphisms between function fields (of arbitrary dimension) over $K$. Next, we apply Theorem $A$ to prove the "injectivity part" (Theorem $C$ ) of the pro- $p$ "Section Conjecture." Finally, in an Appendix, we derive from Theorem $A$ an isomorphism version of the Grothendieck Conjecture for certain hyperbolic surfaces (Theorem $D$ ).

Notation: If $K$ is a field and $X_{K}$ is a $K$-scheme, we denote by $\Pi_{X_{K}}^{\text {prf }} \stackrel{\text { def }}{=} \pi_{1}\left(X_{K}\right)$ the fundamental group of $X_{K}$ (for some choice of base-point), and by $\Gamma_{K}$ the absolute Galois group of $K$. Then we have a natural morphism $\Pi_{X_{K}}^{\mathrm{prf}} \rightarrow \Gamma_{K}$ whose kernel is the geometric fundamental group $\Delta_{X}^{\text {prf }} \subseteq \Pi_{X_{K}}^{\text {prf }}$. Let $\Delta_{X}$ be the maximal pro- $p$ quotient of $\Delta_{X}^{\text {prf }}$. Then the kernel of $\Delta_{X}^{\mathrm{prf}} \rightarrow \Delta_{X}$ is a normal subgroup of $\Pi_{X_{K}}^{\mathrm{prr}}$, so by forming the quotient of $\Pi_{X_{K}}^{\mathrm{prf}}$ by this normal subgroup, we obtain a group $\Pi_{X_{K}}$, together with a morphism $\Pi_{X_{K}} \rightarrow \Gamma_{K}$ whose kernel is $\Delta_{X}$. Next, if $\Delta$ is a topological group, we let: $\Delta\{0\} \stackrel{\text { def }}{=} \Delta$; for $i \geq 1$, $\Delta\{i\} \stackrel{\text { def }}{=}[\Delta\{i-1\}, \Delta\{i-1\}]$ (here we mean "the closed subgroup generated by the purely 
group-theoretic commutator subgroup"). In the specific case of fundamental groups, let us write " $\Pi$ " (respectively, " $\Delta$ ") for $\Pi / \Delta\{i\}$ (respectively, $\Delta / \Delta\{i\}$ ).

Our first main theorem is the following:

Theorem A. Let $p$ be a prime number. Let $K$ be sub-p-adic (cf. Definition 15.4 (i)). Let $X_{K}$ be a smooth pro-variety over $K$. Let $Y_{K}$ be a hyperbolic pro-curve over $K$. Let $\operatorname{Hom}_{K}^{\text {dom }}\left(X_{K}, Y_{K}\right)$ be the set of dominant $K$-morphisms from $X_{K}$ to $Y_{K}$. Let $\operatorname{Hom}_{\Gamma_{K}}^{\text {open }}\left(\Pi_{X_{K}}, \Pi_{Y_{K}}\right)$ (respectively, $\left.\operatorname{Hom}_{\Gamma_{K}}^{\text {open }}\left(\Pi_{X_{K}}^{\mathrm{prf}}, \Pi_{Y_{K}}^{\mathrm{prf}}\right)\right)$, be the set of open, continuous group homomorphisms $\Pi_{X_{K}} \rightarrow \Pi_{Y_{K}}$ (respectively, $\Pi_{X_{K}}^{\mathrm{prf}} \rightarrow \Pi_{Y_{K}}^{\mathrm{prf}}$ ) over $\Gamma_{K}$, considered up to composition with an inner automorphism arising from $\Delta_{Y}$ (respectively, $\left.\Delta_{Y}^{\mathrm{prf}}\right)$. Then the natural maps

$$
\begin{aligned}
\operatorname{Hom}_{K}^{\text {dom }}\left(X_{K}, Y_{K}\right) & \rightarrow \operatorname{Hom}_{\Gamma_{K}}^{\text {open }}\left(\Pi_{X_{K}}^{\mathrm{prf}}, \Pi_{Y_{K}}^{\mathrm{prf}}\right) \\
& \rightarrow \operatorname{Hom}_{\Gamma_{K}}^{\text {open }}\left(\Pi_{X_{K}}, \Pi_{Y_{K}}\right)
\end{aligned}
$$

are bijective.

This Theorem is given as Theorem 16.5 (cf. also the Remark following Theorem 16.5) in the text. It is from Theorem $A$ that all of the other major results of this paper are derived.

We remark that:

(1) In fact, really, the main portion of Theorem $A$ is the bijectivity of the first and third Hom's. That is to say, the bijectivity of the first and second Hom's follows formally from the bijectivity of the first and third Hom's. See the Remark following Theorem 16.5 for more details.

(2) The notion of a "smooth pro-variety" (respectively, "hyperbolic procurve") has as special cases: (i) a smooth variety (respectively, hyperbolic curve) over $K$; (ii) the spectrum of a function field of arbitrary dimension (respectively, function field of dimension one) over $K$.

(3) There exists a substantial body of people who, when they speak of "the Grothendieck Conjecture" (respectively, "the pro- $p$ Grothendieck Conjecture"), refer to the following rather specific statement (which is a special case of the general philosophy discussed in the preceding subsection): If $K$ is a finitely generated extension of $\mathbf{Q}$, and $X_{K}$ and $Y_{K}$ are either hyperbolic curves over $K$ or the spectra of one-dimensional function fields over $K$, then the isomorphisms of $X_{K}$ with $Y_{K}$ are in natural bijective correspondence with the outer isomorphisms over $\Gamma_{K}$ of $\Pi_{X_{K}}^{\text {prf }}$ (respectively, $\Pi_{X_{K}}$ ) with $\Pi_{Y_{K}}^{\text {prf }}$ (respectively, $\Pi_{Y_{K}}$ ). This statement is manifestly a special case of the profinite version - see (1) above (respectively, pro- $p$ version, i.e., version as stated above) of Theorem $A$. 
We also have truncated versions of Theorem $A$ (Theorems 18.1 and 18.2 in the text):

Theorem A'. Let $K$ be sub-p-adic. Let $X_{K}$ be a smooth variety over $K$. Let $Y_{K}$ be a hyperbolic curve over $K$. Let $n \geq 5$. Then every continuous open homomorphism

$$
\theta: \Pi_{X_{K}}^{n} \rightarrow \Pi_{Y_{K}}^{n}
$$

over $\Gamma_{K}$ induces a dominant morphism $\mu: X_{K} \rightarrow Y_{K}$ whose induced morphism on fundamental groups coincides (up to composition with an inner automorphism arising from $\left.\Delta_{Y_{K}}\right)$ with the morphism $\Delta_{X_{K}}^{n-3} \rightarrow \Delta_{Y_{K}}^{n-3}$ defined by considering $\theta$ "modulo $\Delta\{n-3\}$."

Theorem A". $\quad$ Let $K$ be sub-p-adic. Let $X_{K}$ be a smooth pro-variety over $K$. Let $Y_{K}$ be a hyperbolic pro-curve over $K$. Let $n_{0}^{\prime}$ be the minimum transcendence degree over $\mathbf{Q}_{p}$ of all finitely generated field extensions of $\mathbf{Q}_{p}$ that contain $K$. Let $n_{0}^{\prime \prime}$ be the transcendence degree over $K$ of the function field of $X_{K}$. Let $n_{0} \stackrel{\text { def }}{=} n_{0}^{\prime}+2\left(n_{0}^{\prime \prime}-1\right)+1$.

Let $n \geq 3 n_{0}+5$. Then every continuous open homomorphism

$$
\theta: \Pi_{X_{K}}^{n} \rightarrow \Pi_{Y_{K}}^{n}
$$

over $\Gamma_{K}$ induces a dominant morphism $\mu: X_{K} \rightarrow Y_{K}$ whose induced morphism on fundamental groups coincides (up to composition with an inner automorphism arising from $\Delta_{Y_{K}}$ ) with the morphism $\Delta_{X_{K}}^{n-3-3 n_{0}} \rightarrow \Delta_{Y_{K}}^{n-3-3 n_{0}}$ defined by considering $\theta$ "modulo $\Delta\left\{n-3-3 n_{0}\right\} . "$

Our second main theorem (Theorem 17.1 in the text) is the following:

Theorem B. Let $p$ be a prime number. Let $K$ be sub-p-adic. Let $L$ and $M$ be function fields of arbitrary dimension over $K$. (In particular, we assume that $K$ is algebraically closed in $L$ and $M$.) Let $\operatorname{Hom}_{K}(\operatorname{Spec}(L), \operatorname{Spec}(M))$ be the set of $K$-morphisms from $M$ to L. Let $\operatorname{Hom}_{\Gamma_{K}}^{\text {open }}\left(\Gamma_{L}, \Gamma_{M}\right)$ be the set of open, continuous group homomorphisms $\Gamma_{L} \rightarrow \Gamma_{M}$ over $\Gamma_{K}$, considered up to composition with an inner automorphism arising from $\operatorname{Ker}\left(\Gamma_{M} \rightarrow \Gamma_{K}\right)$. Then the natural map

$$
\operatorname{Hom}_{K}(\operatorname{Spec}(L), \operatorname{Spec}(M)) \rightarrow \operatorname{Hom}_{\Gamma_{K}}^{\text {open }}\left(\Gamma_{L}, \Gamma_{M}\right)
$$

is bijective.

Note that in characteristic zero, this generalizes the results of [Pop1], [Pop2], where a similar result to Theorem $B$ is obtained, except that the morphisms $\Gamma_{L} \rightarrow \Gamma_{M}$ are required to be isomorphisms, and $K$ is required to be finitely generated over $\mathbf{Q}$. 
Our third main theorem (Theorem 19.1 in the text) is the following:

Theorem C. Let $p$ be a prime number. Let $K$ be sub-p-adic. Let $X_{K}$ be a hyperbolic curve over $K$. Let $X_{K}(K)$ be the set of $K$-valued points of $X_{K}$. Let $\operatorname{Sect}\left(\Gamma_{K}, \Pi_{X_{K}}\right)$ be the set of sections $\Gamma_{K} \rightarrow \Pi_{X_{K}}$ of $\Pi_{X_{K}} \rightarrow \Gamma_{K}$, considered up to composition with an inner automorphism arising from $\Delta_{X}$. Then the natural map

$$
X_{K}(K) \rightarrow \operatorname{Sect}\left(\Gamma_{K}, \Pi_{X_{K}}\right)
$$

is injective.

Finally, in the Appendix, we use Theorem $A$ to derive the following result:

Theorem D. Let $p$ be a prime number. Let $K$ be sub-p-adic. Let $X_{K}$ and $Y_{K}$ be hyperbolically fibred surfaces (see Definition a2.1 in the Appendix for a precise definition of this term) over $K$. Let $\operatorname{Isom}_{K}\left(X_{K}, Y_{K}\right)$ be the set of $K$-isomorphisms (in the category of $K$-schemes) between $X_{K}$ and $Y_{K}$. Let $\operatorname{Isom}_{\Gamma_{K}}\left(\Pi_{X_{K}}^{\text {prf }}, \Pi_{Y_{K}}^{\text {prf }}\right)$ be the set of continuous group isomorphisms $\Pi_{X_{K}}^{\mathrm{prf}} \rightarrow \Pi_{Y_{K}}^{\mathrm{prf}}$ over $\Gamma_{K}$, considered up to composition with an inner automorphism arising from $\Delta_{Y}^{\mathrm{prf}}$. Then the natural map

$$
\operatorname{Isom}_{K}\left(X_{K}, Y_{K}\right) \rightarrow \operatorname{Isom}_{\Gamma_{K}}\left(\Pi_{X_{K}}^{\mathrm{prf}}, \Pi_{Y_{K}}^{\mathrm{prf}}\right)
$$

is bijective.

\section{Recent Work on the Grothendieck Conjecture:}

In this subsection, we would like to take a brief look at recent work on the Grothendieck Conjecture. We will concentrate only on major and recent (since the early 1980's) developments that relate to the present paper, and we make no pretense of giving a complete history of work on the Grothendieck Conjecture. Before beginning, it is worth pointing out that until the appearance of the present paper (and its earlier version [Mzk2]), it was widely assumed that the base field " $K$ " that appears in the Grothendieck Conjecture should be assumed to be finitely generated over $\mathbf{Q}$. It is not clear precisely why this came to be assumed by most people working in the field, but one possible cause is that the Grothendieck Conjecture appears to have originated as an alternative approach to diophantine geometry ([Groth], [Pop3]). Thus, if one's ultimate aim is applications to diophantine geometry, it is quite natural only to look at global fields (i.e., finitely generated extensions of $\mathbf{Q}$ ), and not at $p$-adic local fields, for instance.

Another reason for the fixation on finitely generated extensions of $\mathbf{Q}$ appears to have been that many people conceived of the Grothendieck Conjecture as an anabelian version 
of the Tate Conjecture (Faltings' theorem - see [Falt3]) for abelian varieties over number fields. In fact, this point of view became so deeply engrained that it gave rise to a tendency for people to try to prove the Grothendieck Conjecture for hyperbolic curves over number fields by deducing it from the Tate Conjecture for abelian varieties over number fields (see, e.g., [Naka3]).

In fact, even important early work - such as [Naka1] on the Grothendieck Conjecture for genus zero hyperbolic curves over number fields, or [Pop1], [Pop2] which treat the birational case - which does not try to deduce the Grothendieck Conjecture from the Tate Conjecture still had a distinctly global flavor, and relied extensively on essentially global techniques. These global techniques ultimately proved to be rather irrelevant to the proof of the main results of the present paper. Nevertheless, what was important (from the point of view of the author) about the work of H. Nakamura was that it established a host of basic techniques for studying the outer Galois action on the (nonabelian) geometric fundamental group of a hyperbolic curve, which was unknown territory to most arithmetic geometers, who were only familiar with the Galois action on the abelian fundamental group of an abelian variety. It was this culture of basic techniques (due to H. Nakamura) which permitted the subsequent development of more powerful approaches to the Grothendieck Conjecture itself, as discussed below.

The next important development (in early 1995) was the work of A. Tamagawa ([Tama]) in which it was shown that the isomorphism class of any affine hyperbolic curve over an absolutely finitely generated field is functorially determined by the outer Galois action on its profinite geometric fundamental group. (In fact, by a relatively straightforward argument ([Mzk1]), it is possible to remove the "affineness" hypothesis in Tamagawa's result, at least in characteristic zero.) Unlike the results of the present paper, Tamagawa's result holds in positive characteristic as well as in characteristic zero. Moreover, Tamagawa's ideas on characterizing those sections of $\pi_{1}\left(X_{K}\right) \rightarrow \Gamma_{K}$ (where $X_{K}$ is a hyperbolic curve over a field $K$ ) that arise from geometric points of $X_{K}$ played a pivotal role in inspiring the author to prove the results of the present paper. Concretely speaking, the influence of these ideas of Tamagawa can be seen in the argument of Section 9 of the present paper.

Finally, we turn to discussing the results of the present paper in a historical context. As observed previously, the results of the present paper are (unlike Tamagawa's results) only valid in characteristic zero. On the other hand, the main advances of Theorem $A$ of the present paper relative to [Tama] are as follows:

(1) The hyperbolic curves involved are allowed to be proper. In fact, we even allow what we call hyperbolic pro-curves.

(2) Instead of dealing with profinite geometric fundamental groups, we deal with pro- $p$ geometric fundamental groups. Pro- $p$ results tend to be stronger than profinite results in the sense that profinite results usually follow immediately from pro- $p$ results (see the Remark following Theorem 16.5 for more details).

(3) Instead of just considering isomorphisms, we allow arbitrary dominant morphisms between the varieties involved. 
(4) The variety "on the left" (in the "Hom $(-,-)$ " of Theorem $A$ ) is allowed to be higher-dimensional, and need not even be hyperbolic.

(5) The base field can be any subfield of a finitely generated extension of $\mathbf{Q}_{p}$, whereas for Tamagawa (in characteristic zero) the base field must be a finitely generated extension of $\mathbf{Q}$.

From the point of view of the author, (5) is the most fundamental and important advance as it is associated with the fact that the techniques of the present paper are fundamentally different from those of both Nakamura and Tamagawa in that they are couched in the world of p-adic Hodge theory. That is to say, the proof of Theorem $A$ may be regarded as an application of the theory of [Falt1] and [BK]. It is precisely the use of these $p$-adic techniques that allowed the author to prove a result which was much stronger (in the above five senses) than the result of [Tama] (in characteristic zero) or [Mzk1]. Moreover, it is the opinion of the author that:

The reason that it took so long for Theorem $A$ to be discovered was the overwhelming prejudice of most people in the field that the Grothendieck Conjecture for hyperbolic curves is an essentially global result, akin to the Tate Conjecture for abelian varieties over number fields. In fact, however, it is much more natural to regard the Grothendieck Conjecture for hyperbolic curves as an essentially local, $p$-adic result that belongs to that branch of arithmetic geometry known as $p$-adic Hodge theory.

Moreover, it is the feeling of the author that, more than the technical details of the statement of Theorem A, it is this fact-i.e., that the Grothendieck Conjecture for hyperbolic curves is best understood not as a global, number-theoretic result, but rather as a result in p-adic Hodge theory - that is the central discovery of this paper.

\section{The Structure of the Proof:}

First, we remark that most of the paper (Sections 1 through 14) is devoted to proving the following technical result:

$(*)^{\text {tech }}$ Suppose that $K$ is a finite extension of $\mathbf{Q}_{p}$. Let $X_{K}$ and $Y_{K}$ be proper hyperbolic curves over $K$. Let $U_{K}$ be the spectrum of the function field of $X_{K}$. Then every continuous surjective homomorphism $\theta: \Pi_{U_{K}} \rightarrow \Pi_{Y_{K}}$ over $\Gamma_{K}$ arises from some geometric morphism $U_{K} \rightarrow$ $Y_{K}$.

Section 15 is devoted to the rather standard technicalities necessary to generalize $(*)^{\text {tech }}$ to the case where $K$ is any subfield of a finitely generated extension of $\mathbf{Q}_{p}$. Section 16 is devoted to carrying out a standard "cutting by hyperplane sections argument" which allows one to replace $U_{K}$ by a higher-dimensional smooth pro-variety (which thus completes 
the proof of Theorem $A$ ). Section 17 is devoted to an induction argument used to derive Theorem $B$ from Theorem $A$. Section 18 discusses how the techniques used to prove Theorem $A$ in fact also give rise to the truncated versions Theorem $A^{\prime}$ and Theorem $A^{\prime \prime}$. Finally, Section 19 reviews a standard argument (already present in the work of $\mathrm{H}$. Nakamura) which allows one to derive Theorem $C$ from Theorem $A$. Thus, the rest of this subsection will be devoted to outlining the proof of $(*)^{\text {tech }}$ in Sections 1 through 14 .

In order to simplify the discussion, let us consider first the following slightly modified version of $(*)^{\text {tech }}$ :

$(*)^{\text {prop }}$ Suppose that $K$ is a finite extension of $\mathbf{Q}_{p}$. Let $X_{K}$ and $Y_{K}$ be proper hyperbolic curves over $K$. Then every continuous surjective homomorphism $\theta: \Pi_{X_{K}} \rightarrow \Pi_{Y_{K}}$ over $\Gamma_{K}$ arises from some geometric morphism $X_{K} \rightarrow Y_{K}$.

(Here, "prop" stands for "proper" - i.e., since, unlike in $(*)^{\text {tech }}$, where $U_{K}$ appeared, in $(*)^{\text {prop }}$ only proper curves appear.) Thus, $(*)^{\text {tech }}$ implies $(*)^{\text {prop }}$. In other words, (up to some standard general nonsense) one may think of $(*)^{\text {tech }}$ as the concatenation of $(*)^{\text {prop }}$ with the following assertion:

$(*)^{\text {iner }}$ Any $\theta: \Pi_{U_{K}} \rightarrow \Pi_{Y_{K}}$ as in $(*)^{\text {tech }}$ necessarily factors through the quotient $\Pi_{U_{K}} \rightarrow \Pi_{X_{K}}$.

(Here, "iner" stands for "inertia" - since the kernel of $\Pi_{U_{K}} \rightarrow \Pi_{X_{K}}$ is generated by inertia groups, so $(*)^{\text {iner }}$ is the assertion that $\theta: \Pi_{U_{K}} \rightarrow \Pi_{Y_{K}}$ always sends inertia groups in $\Pi_{U_{K}}$ to the identity.) We will come back to the issue of $(*)^{\text {iner }}$ later, but for now, let us concentrate on outlining the proof of $(*)^{\text {prop }}$, since it is this which is the core of the paper.

Thus, let us assume that we have been given a continuous surjective homomorphism:

$$
\theta: \Pi_{X_{K}} \rightarrow \Pi_{Y_{K}}
$$

over $\Gamma_{K}$. We would like to somehow manufacture out of $\theta$ a morphism from $X_{K}$ to $Y_{K}$. A natural, naive way to start is the following: First, by replacing $X_{K}$ and $Y_{K}$ by appropriate étale coverings, we may assume that neither is hyperelliptic (see, e.g., Lemma 10.4 (4)). Let us write $D_{X}$ (respectively, $\left.D_{Y}\right)$ for $H^{0}\left(X_{K}, \omega_{X_{K} / K}\right)$ (respectively, $H^{0}\left(Y_{K}, \omega_{Y_{K} / K}\right)$ ), the space of global differentials on $X_{K}$ (respectively, $Y_{K}$ ). Then it is well-known that $X_{K}$ (respectively, $Y_{K}$ ) embeds naturally in the projective space $P_{X} \stackrel{\text { def }}{=} \mathbf{P}\left(D_{X}\right)$ (respectively, $\left.P_{Y} \stackrel{\text { def }}{=} \mathbf{P}\left(D_{Y}\right)\right)$. Moreover, since the $p$-adic étale cohomology of $X_{\bar{K}}$ or $Y_{\bar{K}}$ can be computed as the group cohomology of the respective pro- $p$ geometric fundamental group, it follows that the surjection $\theta$ induces a $\Gamma_{K}$-equivariant injection

$$
\theta_{H}: H^{1}\left(Y_{K}, \mathbf{Q}_{p}\right) \hookrightarrow H^{1}\left(X_{K}, \mathbf{Q}_{p}\right)
$$


Moreover, it is well-known (see, e.g., [Falt1], [Tate]) that the $\Gamma_{K}$-module $H^{1}\left(X_{K}, \mathbf{Q}_{p}\right)$ is Hodge-Tate, and, moreover, that if we tensor $H^{1}\left(X_{K}, \mathbf{Q}_{p}\right)$ over $\mathbf{Q}_{p}$ with $\mathbf{C}_{p}(1)$ (where the "(1)" is a "Tate twist"), and take $\Gamma_{K}$-invariants, we naturally recover the space $D_{X}$ of differentials. Thus, since $\theta_{H}$ is $\Gamma_{K}$-equivariant, if we tensor $\theta_{H}$ over $\mathbf{Q}_{p}$ with $\mathbf{C}_{p}(1)$ and take $\Gamma_{K}$-invariants, we obtain from $\theta$ (in a natural way) a $K$-linear injection

$$
\theta_{D}: D_{Y} \hookrightarrow D_{X}
$$

and hence a rational map from $P_{X}$ to $P_{Y}$.

Thus, in some sense, without doing anything terribly new (i.e., we have only just applied results known to Tate since the 1960's), we have already come relatively close to constructing a morphism from $X_{K}$ to $Y_{K}$. Indeed, what we have done is to construct a morphism $\theta_{D}$ from differentials on $Y_{K}$ to differentials on $X_{K}$, which we would like to hope arises as the pull-back map on differentials associated to a morphism from $X_{K}$ to $Y_{K}$. Moreover, since $X_{K}$ (respectively, $Y_{K}$ ) is canonically embedded in $P_{X}$ (respectively, $P_{Y}$ ), it follows from elementary algebraic geometry that the "only" thing we need to show is that $\theta_{D}$ preserves relations: That is, if $i$ is a positive integer, let $D_{X}^{i} \stackrel{\text { def }}{=} H^{0}\left(X_{K}, \omega_{X_{K} / K}^{\otimes i}\right)$. Similarly, we have $D_{Y}^{i}$. Note that multiplication of differential forms defines a natural morphism

$$
\bigotimes D_{Y} \rightarrow D_{Y}^{i}
$$

Let us denote the kernel of this morphism by $\mathcal{R}_{i}$. Thus, $\mathcal{R}_{i}$ is the set of relations (of degree i) defining $Y_{K}$ as a subvariety of $P_{Y}$. Moreover, by composing the $i^{\text {th }}$ tensor power of $\theta_{D}$ with the natural morphism from the $i^{t h}$ tensor power of $D_{X}$ to $D_{X}^{i}$, we obtain a morphism

$$
\kappa^{i}: \bigotimes_{Y}^{i} D_{Y} D_{X}^{i}
$$

Then we shall say that $\theta: \Pi_{X_{K}} \rightarrow \Pi_{Y_{K}}$ preserves relations if $\kappa^{i}\left(\mathcal{R}_{i}\right)=0$ for all positive integers $i$. As stated earlier, once we know that $\theta$ preserves relations, it follows from elementary algebraic geometry (see [Harts], Chapter II) that the rational map from $P_{X}$ to $P_{Y}$ defined by $\theta_{D}$ induces a (dominant) morphism $X_{K} \rightarrow Y_{K}$, as desired. (Once one has this morphism $X_{K} \rightarrow Y_{K}$, the fact that the map that it induces on fundamental groups coincides with $\theta$ is a matter of general nonsense - for details, we refer to the argument preceding Theorem 14.1.)

Thus, to review what we have done so far, we have reduced the main problem to showing that any $\theta: \Pi_{X_{K}} \rightarrow \Pi_{Y_{K}}$ as in (*) prop preserves relations. It should be emphasized at this point, that so far everything that we have done has been painless general nonsense - the substantive mathematical core of the argument is yet to come (after some more general nonsense in the next few paragraphs). The next step is to introduce a field $L$, 
as follows: Let us assume for simplicity that $X_{K}$ extends to a stable curve $\mathcal{X}$ over $\mathcal{O}_{K}$. Let $\mathfrak{p} \in \mathcal{X}$ be an irreducible component of the special fiber of $\mathcal{X}$. Then the completion of the local ring $\mathcal{O}_{\mathcal{X}, \mathfrak{p}}$ is a $p$-adically complete discrete valuation ring $\mathcal{O}_{L}$, whose quotient field we denote by $L$. The space $\Omega_{L}$ of $p$-adically continuous differentials of $L$ over $K$ is then a one-dimensional $L$-vector space. Thus, $L$ is, in some sense, one-dimensional over $K$. Alternatively, one may think of $\operatorname{Spec}(L)$ roughly as some sort of small $p$-adic open set in $X_{K}$. At any rate, from the construction of $L$, it follows that we have a natural $L$-valued point $\xi_{X}: \operatorname{Spec}(L) \rightarrow X_{K}$. Since $L$ is "one-dimensional," it is easy to check that the restriction map on differentials

$$
D_{X}^{i} \rightarrow \Omega_{L}^{\otimes i}
$$

(where " $\Omega_{L}^{\otimes i}$ " denotes the tensor product of $i$ copies of $\Omega_{L}$ over $L$ ) is injective. Thus, instead of checking that $\kappa^{i}\left(\mathcal{R}_{i}\right)=0$, it suffices to check that the composite

$$
\kappa_{L}^{i}: \bigotimes^{i} D_{Y} \rightarrow \Omega_{L}^{\otimes i}
$$

of $\kappa^{i}$ with the above restriction map vanishes on $\mathcal{R}_{i}$. In other words, we would like to compute $\kappa_{L}^{i}$.

To "compute" $\kappa_{L}^{i}$, we need to go back to looking at fundamental groups. First of all, let us observe that by functoriality of the fundamental group, the natural $L$-valued point $\xi_{X}: \operatorname{Spec}(L) \rightarrow X_{K}$ defines a morphism

$$
\alpha_{X}^{L}: \Gamma_{L} \rightarrow \Pi_{X_{K}}
$$

whose composite with the augmentation $\Pi_{X_{K}} \rightarrow \Gamma_{K}$ is the morphism $\Gamma_{L} \rightarrow \Gamma_{K}$ on Galois groups induced by the inclusion of fields $K \subseteq L$. Moreover, if we compose $\alpha_{X}^{L}$ with $\theta$, we obtain a morphism

$$
\alpha_{Y}^{L}: \Gamma_{L} \rightarrow \Pi_{Y_{K}}
$$

Now let us suppose that we know that $\alpha_{Y}^{L}$ is geometric, i.e., that it arises from some $L$ valued point $\xi_{Y}: \operatorname{Spec}(L) \rightarrow Y_{K}$ of $Y_{K}$. Then it follows immediately from the theory of [Falt1] that $\kappa_{L}^{i}$ may be computed as the restriction map on differentials associated to $\xi_{Y}$. But it is clear that this restriction map on differentials annihilates $\mathcal{R}_{i}$. Thus, to summarize: in order to show that $\theta$ preserves relations, it suffices to show that $\alpha_{Y}^{L}$ is geometric.

As remarked earlier, the above prefatory remarks are just "general nonsense." The mathematical core of the present paper lies in showing that given a geometric $\alpha_{X}^{L}$, together with a $\theta: \Pi_{X_{K}} \rightarrow \Pi_{Y_{K}}$ as in (*) ${ }^{\text {prop }}$, the resulting $\alpha_{Y}^{L}$ is again geometric. The proof that $\alpha_{Y}^{L}$ is geometric is long and intricate, and can be divided roughly into four parts: 
(1) First, we consider a $K$-valued point $x \in X_{K}(K)$ of $X_{K}$, and its associated map on fundamental groups $\alpha_{X}^{K}: \Gamma_{K} \rightarrow \Pi_{X_{K}}$. It is elementary to show that the process of passing from the point $x \in X_{K}(K)$ to the arithmetic first Chern class of its associated line bundle

$$
\eta_{X}^{K} \stackrel{\text { def }}{=} c_{1}\left(\mathcal{O}_{X_{K}}(x)\right) \in H_{\text {et }}^{2}\left(X_{K}, \mathbf{Z}_{p}(1)\right)=H^{2}\left(\Pi_{X_{K}}, \mathbf{Z}_{p}(1)\right)
$$

can be carried out just by working with $\alpha_{X}^{K}$ (see the first half of Section 7 for details on this "group-theoretic recipe" for concocting $\eta_{X}^{K}$ out of $\alpha_{X}^{K}$ ). On the other hand, by composing $\alpha_{X}^{K}$ with $\theta$, we get an $\alpha_{Y}^{K}: \Gamma_{K} \rightarrow \Pi_{Y_{K}}$. The first step then is to show that: If one carries out this recipe for $\alpha_{Y}^{K}$ (which may or may not arise geometrically) so as to obtain a class $\eta_{Y}^{K} \in H^{2}\left(\Pi_{Y_{K}}, \mathbf{Z}_{p}(1)\right)$, then $\eta_{Y}^{K}$ is the arithmetic first Chern class of a line bundle (necessarily of degree prime to $p$ ) on $Y_{K}$. This is the topic of Sections 1 through 5 .

(2) Next, we go back to $L$-valued points, and their associated $\alpha_{X}^{L}, \alpha_{Y}^{L}$. Then, just as in the case of $K$-valued points, one can form $\eta_{Y}^{L}$, and one would like to know that $\eta_{Y}^{L}$ is the arithmetic first Chern class of a line bundle (necessarily of degree prime to $p$ ) on $Y_{L} \stackrel{\text { def }}{=} Y_{K} \otimes_{K} L$. This is technically much more intricate than the $K$-valued case, and is done (roughly speaking) by thinking about the "difference" between the Chern class of a $K$-valued point and of an $L$-valued point, and showing that this difference has special properties that are preserved by $\theta$. This allows one to derive the assertion in the $L$-valued case from the corresponding assertion in the $K$-valued case, which was already handled in (1) above. This reduction is the topic of Sections 6 and 7.

(3) Recall that we would like to show that $\alpha_{Y}^{L}$ is geometric. We know from (2) above that at least there exists a line bundle on $Y_{L}$ of degree prime to $p$. Then an elementary algebraic geometry argument shows that this implies the existence of a rational point of $Y_{L}$ defined over a tamely ramified (this will be crucial in Step (4)!) extension $L^{\prime}$ of $L$. Although this portion of the proof is technically rather trivial, its discovery was a key step in the creation of the proof of Theorem $A$. This portion of the proof is discussed in Section 8.

(4) Finally, by applying (3) to all the curves in a certain tower of coverings of the original $Y_{L}$, we obtain a collection of rational points of the curves of this tower that are defined over tamely ramified extensions of $L$. Then by using Faltings' $p$-adic Hodge theory ([Falt1]), we show that these rational points necessarily converge $p$-adically to a single $L$-valued point of $Y_{L}=Y_{K} \otimes_{K} L$ whose associated $\Gamma_{L} \rightarrow \Pi_{Y_{K}}$ is necessarily equal to 
$\alpha_{Y}^{L}$. This completes the proof of the geometricity of $\alpha_{Y}^{L}$. This portion of the proof is given in Sections 9 and 10 (and applied in Section 13).

As discussed above, once one knows the geometricity of $\alpha_{Y}^{L}$, one can conclude the preservation of relations - this is discussed in Sections 11 through 13 . Finally, the proof of $(*)^{\text {iner }}$ (i.e., the difference between $(*)^{\text {tech }}$ and $\left.(*)^{\text {prop }}\right)$ is given in Section 14. Unfortunately, in order to show $(*)^{\text {iner }}$, it is necessary to go through all the steps discussed so far for a given $\theta: \Pi_{U_{K}} \rightarrow \Pi_{Y_{K}}$, and then to conclude $(*)^{\text {iner }}$ from the preservation of relations. This makes the proof much more technically intricate than it would be if one could prove $(*)^{\text {iner }}$ from some sort of a priori argument, and then prove preservation of relations only for $\theta: \Pi_{X_{K}} \rightarrow \Pi_{Y_{K}}$ as in $(*)^{\text {prop }}$.

We would like to close this outline of the proof of Theorem $A$ by discussing Step (1) above (i.e., the content of Sections 1 through 5) in greater detail. The reason for this is that Step (1) is what allowed us to generalize the "isomorphisms only" result of [Mzk2] to the homomorphism result of the present paper. First of all, let us recall the notion of the Malčev completion (cf., e.g., [Del], §9) of $\Delta_{X}$. In fact, we shall only need the "smallest nontrivial part of Malčev completion": concretely, a unipotent algebraic group over $\mathbf{Q}_{p}$, which we denote by $M_{X}$, whose representations are the same as continuous representations of $\Delta_{X} /\left[\Delta_{X},\left[\Delta_{X}, \Delta_{X}\right]\right]$ on some $\mathbf{Q}_{p}$-vector space $V$ which is equipped with a $\Delta_{X}$-invariant filtration on whose subquotients $\Delta_{X}$ acts trivially. In fact, since unipotent algebraic groups are equivalent to their Lie algebras, we shall consider instead the Lie algebra $\mathfrak{M}_{X}$ of $M_{X}$.

Next, observe that any section $\alpha_{X}^{K}: \Gamma_{K} \rightarrow \Pi_{X_{K}}$ defines (by conjugation) a true action (i.e., not just an action up to inner automorphisms) of $\Gamma_{K}$ on $\Delta_{X}$, hence on $\mathfrak{M}_{X}$. Relative to this action, their exists a unique "weight zero quotient" $\mathfrak{M}_{X} \otimes_{\mathbf{Q}_{p}} \mathbf{C}_{p} \rightarrow \mathfrak{Z}_{X}$. Here, we call the quotient "weight zero" because it is the maximal quotient $\mathfrak{M}_{X} \otimes_{\mathbf{Q}_{p}} \mathbf{C}_{p} \rightarrow Q$ for which the action of $\Gamma_{K}$ on $Q$ is such that $Q$ has a filtration by $\Gamma_{K}$-submodules whose subquotients are $\Gamma_{K}$-equivariantly isomorphic to $\mathbf{C}_{p}$. Moreover, this quotient $\mathfrak{M}_{X} \otimes_{\mathbf{Q}_{p}} \mathbf{C}_{p} \rightarrow \mathfrak{Z}_{X}$ is independent of the choice of section $\alpha_{X}^{K}$.

Step (1) is based on the following pair of observations:

(i) On the one hand, if $\alpha_{X}^{K}$ arises geometrically, then $\mathfrak{Z}_{X}$ is "HodgeTate." (By abuse of terminology, we use the term "Hodge-Tate" here to mean that $\mathfrak{Z}_{X}$ has a $\mathbf{C}_{p}$-basis of $\Gamma_{K}$-invariant elements.) This is the content of Proposition 3.5.

(ii) On the other hand, if $\mathfrak{Z}_{X}$ is "Hodge-Tate," then the class $\eta_{X}^{K}$ is the first Chern class of a line bundle. This is essentially the content of the calculation performed in Proposition 4.4 (see also Lemma 7.3).

Moreover, if one starts with a surjective $\theta: \Pi_{X_{K}} \rightarrow \Pi_{Y_{K}}$, then $\mathfrak{Z}_{X}$ maps naturally to, and in fact, surjects onto $\mathfrak{Z}_{Y}$. Thus, if one starts with an $\alpha_{X}^{K}$ that arises geometrically, then one knows from Observation (i) above that $\mathfrak{Z}_{X}$ is Hodge-Tate, but any quotient of a Hodge-Tate representation of $\Gamma_{K}$ - i.e., such as $\mathfrak{Z}_{Y}$ - is always Hodge-Tate, so Observation (ii) above thus allows one to complete Step (1). 
At this point, the reader may wonder how the author stumbled upon the two key observations of the preceding paragraph. In fact, the author first realized what was going on by considering the ordinary case (see Section 1 for a detailed discussion). In this case, the "weight zero quotient" exists at the level of groups, without passing to Malčev completions or tensoring with $\mathbf{C}_{p}$ : namely, (in the notation of Section 1) it is the quotient $\Delta_{X} \rightarrow \Delta_{X}^{\text {et }}$ (which thus gives rise to a quotient $\Pi_{X_{K}} \rightarrow \Pi_{X_{K}}^{\mathrm{et}}$ ). Moreover, as is shown in Section 1, it is elementary to show that $\Delta_{X} \rightarrow \Delta_{X}^{\text {et }}$ can be recovered group-theoretically, and that every section $\Gamma_{K} \rightarrow \Pi_{X_{K}}$ that arises geometrically (from a point of $X_{K}$ ) induces a fixed, group-theoretically constructible section $\theta_{X}: \Gamma_{K} \rightarrow \Pi_{X_{K}}^{\mathrm{et}}$ of $\Pi_{X_{K}}^{\mathrm{et}} \rightarrow \Gamma_{K}$. These observations are enough to complete Step (1) (in the ordinary case). Thus, one may regard the discussion in the preceding paragraphs (which is valid in the nonordinary case) as simply the result of generalizing the observations discussed in this paragraph in the ordinary case to the possibly nonordinary case by means of the technical machinery of the Malčev completion and $p$-adic Hodge theory.

Finally, let us make the following observation: As one can see from the key argument discussed above, in fact, really, one does not need all of $\Delta_{X}$. That is to say, in the above argument (concerning the issue of when $\mathfrak{Z}_{X}$ is Hodge-Tate), one actually only uses the quotient $\Delta_{X} /\left[\Delta_{X},\left[\Delta_{X}, \Delta_{X}\right]\right]$. It is this observation that is behind the truncated generalizations (Theorems $A^{\prime}$ and $A^{\prime \prime}$ ) of Theorem $A$.

\section{Acknowledgements:}

I would like to thank A. Tamagawa: (i) for inspiring me by means of his paper [Tama]; (ii) for numerous discussions during which I presented the proof of the main results of the present paper; (iii) for advice on surmounting two technical problems (see the Remark at the end of Section 12; Lemmas 15.6 and 15.8); (iv) for explaining to me the "general nonsense argument" preceding Theorem 14.1; (v) for explaining to me basic facts concerning the Malčev completion, which were of immense importance in developing the theory discussed in this paper. Also, I would like to thank T. Saito and G. Faltings for pointing out various minor errors in earlier versions of this manuscript.

\section{Section 0: Preliminaries and Notations}

Let $p$ be a prime number. Throughout this paper the symbol " $\wedge$ " over or to the upperright of an object will denote the p-adic completion of that object. Let $K$ be a $\mathbf{Q}_{p}$-algebra. Let $\Omega$ be an algebraically closed field. Then given a base-point $b \in \operatorname{Hom}_{\operatorname{Ring}}(K, \Omega)$, we can form the algebraic fundamental group

$$
\pi_{1}(\operatorname{Spec}(K), b)
$$

Typically, the choice of base-point $b$ will not be important for us, so we shall write $\Gamma_{K}$ for $\pi_{1}(\operatorname{Spec}(K), b)$. Suppose that $\operatorname{Spec}(\bar{K})$ is a "universal covering space" for $\operatorname{Spec}(K)$ such 
that $b$ arises from a ring homomorphism $\bar{K} \rightarrow \Omega$. Then we may think of $\Gamma_{K}$ as $\operatorname{Gal}(\bar{K} / K)$. We shall denote the étale cohomology of $\operatorname{Spec}(K)$ by $H^{i}(K,-)$. If $M$ is a profinite abelian group with a continuous $\Gamma_{K}$-action, then $M$ naturally defines an inverse system $\left\{\mathcal{F}_{\alpha}\right\}$ of locally constant sheaves on the étale site of $\operatorname{Spec}(K)$, and we shall write

$$
H^{i}(K, M)
$$

for the inverse limit (over $\alpha$ ) of the $H^{i}\left(K, \mathcal{F}_{\alpha}\right)$. Note that for each $r \in \mathbf{Z}, \mathbf{Z}_{p}(r)$ (where the " $(r)$ " is a Tate twist) has a natural structure of $\Gamma_{K}$-module.

Definition 0.1. We shall call $K$ a $p$-adic field if it is the quotient field of a $p$-adically complete, mixed characteristic discrete valuation $\operatorname{ring} \mathcal{O}_{K}$. We shall denote the residue field (respectively, maximal ideal) of $\mathcal{O}_{K}$ by $k$ (respectively, $\mathfrak{m}_{K}$ ). We shall call $K$ a $p$-adic local field if $k$ is a finite field.

If $K$ is a $p$-adic field, and $\bar{K}$ is an algebraic closure of $K$, then $K \hookrightarrow \Omega \stackrel{\text { def }}{=} \bar{K}$ determines a base-point " $b$," and we have $\Gamma_{K}=\operatorname{Gal}(\bar{K} / K)$. In this case, $H^{i}(K,-)$ is equal to the continuous group cohomology of the profinite group $\Gamma_{K}$. If $K$ is a $p$-adic local field, then $K$ is, in fact, a finite extension of $\mathbf{Q}_{p}$.

Now (without any assumptions on the $\mathbf{Q}_{p}$-algebra $K$ ), let us assume that we are given a hyperbolic curve $X_{K} \rightarrow \operatorname{Spec}(K)$ over $K$ of type $(g, r)$. (By "curve," we shall always mean a smooth, one-dimensional, geometrically connected scheme over the base. By "type $(g, r)$," we mean that $X_{K} \otimes_{K} \bar{K}$ is obtained by removing $r$ mutually nonintersecting $\bar{K}$ valued points from a proper curve over $\bar{K}$ of genus $g$. By "hyperbolic," we mean that $2 g-2+r \geq 1$.) When $X_{K}$ is proper, we shall denote its Jacobian (an abelian scheme over $K$ ) by $J_{X}$ (or $J_{X_{K}}$ when several bases are in use and it is necessary to specify the base in question).

Let us assume that $X_{K}$ is equipped with a base-point $x \in X_{K}(\Omega)$ (which is compatible with the base-point $b$ of $\operatorname{Spec}(K))$. Then we can form $\Pi_{X}^{\text {prf }} \stackrel{\text { def }}{=} \pi_{1}\left(X_{K}, x\right)$ and $\Delta_{X}^{\text {prf }} \stackrel{\text { def }}{=}$ $\pi_{1}\left(X_{\bar{K}}, x\right)$. (The use of " $\Delta$ " to denote the geometric fundamental group may be new to some readers. Here, we use " $\Delta$ " partly because " $\Pi$ " is already used for the arithmetic fundamental group and partly to conform to the notations of [Falt1], a reference on which the present paper depends heavily.) Occasionally, to avoid confusion, we shall also use the notation $\Delta_{X_{K}}^{\mathrm{prf}}$ for $\Delta_{X}^{\mathrm{prf}}$. Let $\Delta_{X}$ be the maximal pro- $p$ quotient of $\Delta_{X}^{\mathrm{prf}}$. Since the kernel of $\Delta_{X}^{\mathrm{prf}} \rightarrow \Delta_{X}$ is normal in $\Pi_{X_{K}}^{\mathrm{prf}}$, we may form the quotient of $\Pi_{X_{K}}^{\mathrm{prf}}$ by this kernel, and call the resulting quotient group $\Pi_{X_{K}}$. Thus, we have an exact sequence

$$
1 \rightarrow \Delta_{X} \rightarrow \Pi_{X_{K}} \rightarrow \Gamma_{K} \rightarrow 1
$$

Moreover, this exact sequence induces a representation 


$$
\rho_{X}: \Gamma_{K} \rightarrow \operatorname{Out}\left(\Delta_{X}\right) \stackrel{\text { def }}{=} \operatorname{Aut}\left(\Delta_{X}\right) / \operatorname{Inn}\left(\Delta_{X}\right)
$$

into the outer automorphism group of $\Delta_{X}$. (Here, "Aut $\left(\Delta_{X}\right)$ " (respectively, "Inn $\left(\Delta_{X}\right)$ ") denotes the group of continuous automorphisms (respectively, inner automorphisms) of $\Delta_{X}$.) Conversely, it is well-known (see, e.g., [Tama], §7, A.) that the above exact sequence can be recovered from $\rho_{X}$.

Next, we would like to introduce some terminology particular to pro- $p$ groups. Let $\Delta$ be a pro-p group (i.e., a topological group obtained by taking an inverse limit of finite groups of $p$-power order). Let $\Delta^{\prime} \subseteq \Delta$ be the unique normal subgroup of $\Delta$ with the following property: $\Delta \rightarrow \Delta / \Delta^{\prime}$ is the maximal (topologically) Hausdorff abelian quotient of $\Delta$ which is annihilated by $p$. For $i \geq 0$, let $\Delta^{<0>} \stackrel{\text { def }}{=} \Delta ; \Delta^{<i+1>} \stackrel{\text { def }}{=}\left(\Delta^{<i>}\right)^{\prime}$. Thus, we obtain a descending series of closed normal (even characteristic!) subgroups $\ldots \subseteq \Delta^{<i>} \subseteq \ldots \subseteq \Delta$. Note that since $\Delta$, being a pro- $p$ group, is "pro-solvable," it follows that the intersection of all the $\Delta^{<i>}$ is $\{1\}$. Moreover, if $\Delta$ is topologically finitely generated, it follows that the $\Delta / \Delta^{<i>}$ are all finite groups.

Definition 0.2. We shall refer to any one of the $\Delta^{<i>}$ as a $p$-derivate of $\Delta$.

Thus, in particular, if $\Delta=\Delta_{X}$, then it follows (by the structure of the fundamental group of an algebraic curve in characteristic zero) that $\Delta$ is topologically finitely generated, so the $\Delta / \Delta^{<i>}$ are all finite groups.

Next, let us consider the Kummer sequence on $X_{K}$, i.e., the exact sequence of étale sheaves on $X_{K}$ given by $0 \rightarrow \mathbf{Z} / p^{n} \mathbf{Z}(1) \rightarrow \mathbf{G}_{m} \rightarrow \mathbf{G}_{m} \rightarrow 0$ (for $n \geq 1$ ). (Here, the "(1)" is a "Tate twist," and the morphism from $\mathbf{G}_{m}$ to $\mathbf{G}_{m}$ is given by raising to the $\left(p^{n}\right)^{t h}$ power.) The connecting morphism induced on étale cohomology by the Kummer sequence then gives us a morphism $H^{1}\left(X_{K}, \mathbf{G}_{m}\right) \rightarrow H^{2}\left(X_{K},\left(\mathbf{Z} / p^{n} \mathbf{Z}\right)(1)\right)$. Now suppose that $\mathcal{L}$ is a line bundle on $X_{K}$. Applying the connecting morphism just considered to $\mathcal{L}$ (which defines an element of $H^{1}\left(X_{K}, \mathbf{G}_{m}\right)$ ), we obtain a compatible system of classes in $H^{2}\left(X_{K},\left(\mathbf{Z} / p^{n} \mathbf{Z}\right)(1)\right)($ for each $n \geq 1)$, hence a class $c_{1}(\mathcal{L}) \in H^{2}\left(X_{K}, \mathbf{Z}_{p}(1)\right)$.

Definition 0.3. We shall refer to $c_{1}(\mathcal{L})$ as the arithmetic first Chern class of $\mathcal{L}$.

Finally, we have the following elementary technical result, which states that the étale cohomology of a hyperbolic curve may be computed as the group cohomology of its fundamental group:

Lemma 0.4. Assume that $K$ is a field. For all integers $i, r$, the natural morphisms

$$
H^{i}\left(\Delta_{X}, \mathbf{Z}_{p}(r)\right) \rightarrow H^{i}\left(X_{\bar{K}}, \mathbf{Z}_{p}(r)\right) ; \quad H^{i}\left(\Delta_{X_{K} \times_{K} X_{K}}, \mathbf{Z}_{p}(r)\right) \rightarrow H^{i}\left(X_{\bar{K}} \times_{\bar{K}} X_{\bar{K}}, \mathbf{Z}_{p}(r)\right)
$$


and

$$
H^{i}\left(\Pi_{X_{K}}, \mathbf{Z}_{p}(r)\right) \rightarrow H^{i}\left(X_{K}, \mathbf{Z}_{p}(r)\right) ; \quad H^{i}\left(\Pi_{X_{K} \times_{K} X_{K}}, \mathbf{Z}_{p}(r)\right) \rightarrow H^{i}\left(X_{K} \times_{K} X_{K}, \mathbf{Z}_{p}(r)\right)
$$

are isomorphisms.

Proof. By the Leray-Serre spectral sequence, it suffices to prove that the morphisms of the first line are isomorphisms. Let $Z$ be $X_{\bar{K}}$ or $X_{\bar{K}} \times_{\bar{K}} X_{\bar{K}}$. Then it follows by general nonsense that it suffices to check that for any finite étale Galois covering $Y \rightarrow Z$ of $p$-power order, and any cohomology class $\eta \in H^{i}\left(Y, \mathbf{F}_{p}\right)$ (where $i>0$ ), there exists a finite étale covering $Y^{\prime} \rightarrow Y$ of $p$-power order such that $\left.\eta\right|_{Y^{\prime}}=0$. By the Künneth formula, it suffices to do the case $Z=X_{\bar{K}}$. Then what we must check is trivial for $i>2$ (since then $\eta=0$ to begin with), and clear for $i=1$ (by the relationship between étale coverings and $H^{1}$ ). If $i=2$, then it suffices to take $Y^{\prime} \rightarrow Y$ such that $Y^{\prime} \rightarrow Y$ has degree $p$ over every connected component of $Y$. This completes the proof.

\section{Section 1: The Ordinary Case}

Let $p$ be a prime number. Let $K$ be a $p$-adic field with algebraically closed residue field. Then, as discussed in Section 0, the absolute Galois group of $K$ will be denoted $\Gamma_{K}$. Let $X_{K} \rightarrow \operatorname{Spec}(K)$ be a hyperbolic curve over $K$ of type $(g, r)$. In this Section, let us also assume that $X_{K}$ admits a stable extension $X \rightarrow \operatorname{Spec}\left(\mathcal{O}_{K}\right)$ over $\mathcal{O}_{K}$. By this, we mean that there exists a (necessarily unique) r-pointed stable (in particular, proper) curve $\bar{X} \rightarrow \operatorname{Spec}\left(\mathcal{O}_{K}\right)$ of genus $g$ such that $X$ is the complement in $\bar{X}$ of the images of the $r$ marking sections of $\bar{X}$. Let us write $X_{k}$ (respectively, $\bar{X}_{k}$ ) for $X \otimes_{\mathcal{O}_{K}} k$ (respectively, $\left.\bar{X} \otimes \mathcal{O}_{K} k\right)$.

Remark. Recall that an $r$-pointed stable curve of genus $g$ (where $2 g-2+r \geq 1$ ) is a proper, flat morphism $f: C \rightarrow S$, together with $r$ mutually disjoint sections $\sigma_{1}, \ldots, \sigma_{r}: S \rightarrow C$, such that

(1) The geometric fibers of $f$ are connected, reduced, of arithmetic genus $g$, and have at most nodes as singularities.

(2) The sheaf $\omega_{C / S}\left(\sigma_{1}+\ldots+\sigma_{r}\right)$ (i.e., the sheaf of sections of the dualizing bundle of $C$ over $S$ with poles of order $\leq 1$ at the divisors defined by the images of the sections $\left.\sigma_{1}, \ldots, \sigma_{r}\right)$ is relatively ample over $S$.

We refer to $[\mathrm{DM}]$, Knud] for more details. 
Now we make the following

Definition 1.1. We shall call $X_{K}$ ordinary if the Jacobian of every connected component of the normalization of the curve $\bar{X}_{k}$ is an ordinary abelian variety.

In this Section, we would like to assume that $X_{K}$ is ordinary. Under this assumption, it is well-known that $\pi_{1}\left(\bar{X}_{k}\right)^{(p)}$ (where the " $(p)$ " denotes the maximal pro- $p$ quotient) is a free pro-p group of rank $g$. (Indeed, this follows from the fact that $H_{\mathrm{et}}^{2}\left(\bar{X}_{k}, \mathbf{F}_{p}\right)=0$ (which may be shown by using the long exact sequence in étale cohomology obtained by considering $\mathbf{F}_{p}$ as the kernel of " $1-$ Frobenius" acting on $\mathcal{O}_{\bar{X}_{k}}$ ), plus Proposition 2.3 of Chapter III, $\S 3$, of [Sha].) Moreover, since étale coverings of $\bar{X}_{k}$ lift uniquely to characteristic zero, it follows that we have a continuous surjection:

$$
\epsilon_{X}: \Pi_{X_{K}} \rightarrow \pi_{1}\left(\bar{X}_{k}\right)^{(p)}
$$

If we restrict $\epsilon_{X}$ to $\Delta_{X}$, we obtain a surjection $\Delta_{X} \rightarrow \pi_{1}\left(\bar{X}_{k}\right)^{(p)}$. In the following, we shall regard $\pi_{1}\left(\bar{X}_{k}\right)^{(p)}$ as a quotient of $\Delta_{X}$ via this surjection. Let us denote this quotient by $\Delta_{X}^{\text {et }}$. Note that the kernel of the surjection $\Delta_{X} \rightarrow \Delta_{X}^{\text {et }}$ is normal as a subgroup of $\Pi_{X_{K}}$. Thus, by taking the quotient of $\Pi_{X_{K}}$ by this kernel, we obtain a quotient $\Pi_{X_{K}} \rightarrow \Pi_{X_{K}}^{\mathrm{et}^{K}}$. In other words, we have an exact sequence

$$
1 \rightarrow \Delta_{X}^{\mathrm{et}} \rightarrow \Pi_{X_{K}}^{\mathrm{et}} \rightarrow \Gamma_{K} \rightarrow 1
$$

together with a surjection

$$
\zeta_{X}: \Pi_{X_{K}}^{\mathrm{et}} \rightarrow \Delta_{X}^{\mathrm{et}}
$$

which is the identity on $\Delta_{X}^{\text {et }}$.

Now observe that the kernel of $\zeta_{X}$ projects isomorphically to $\Gamma_{K}$. Thus, we obtain a section

$$
\theta_{X}: \Gamma_{K} \rightarrow \Pi_{X_{K}}^{\mathrm{et}}
$$

Next, let us observe that every element of $\operatorname{Im}\left(\theta_{X}\right)$ commutes with every element of $\Delta_{X}^{\text {et }}$. Indeed, this follows from the fact that such commutation relations hold after projection by $\Pi_{X_{K}}^{\text {et }} \rightarrow \Gamma_{K}$, plus the fact that $\operatorname{Im}\left(\theta_{X}\right)=\operatorname{Ker}\left(\zeta_{X}\right)$ is normal in $\Pi_{X_{K}}^{\text {et }}$ (and maps isomorphically to $\Gamma_{K}$ via the projection $\left.\Pi_{X_{K}}^{\mathrm{et}} \rightarrow \Gamma_{K}\right)$. On the other hand, since, as is well-known (see, e.g., [Tama], $\S 1$, Propositions 1.1, 1.11), pro- $p$ free groups (of rank $\geq 2$ ) have trivial centers, it thus follows that we obtain the following "group-theoretic" characterization of $\theta_{X}$ (when $\left.g \geq 2\right)$ : 
Lemma 1.2. Suppose that $g \geq 2$. Then the section $\theta_{X}: \Gamma_{K} \rightarrow \Pi_{X_{K}}^{\mathrm{et}}$ of $\Pi_{X_{K}}^{\mathrm{et}} \rightarrow \Gamma_{K}$ is the unique section whose image commutes with every element of $\Delta_{X}^{\mathrm{et}} \subseteq \Pi_{X_{K}}^{\mathrm{et}}$.

On the other hand, the quotient $\Delta_{X} \rightarrow \Delta_{X}^{\text {et }}$ (and hence also the quotient $\Pi_{X_{K}} \rightarrow$ $\Pi_{X_{K}}^{\mathrm{et}}$ ) can also be reconstructed group-theoretically by means of the following condition on an open normal subgroup $H \subseteq \Delta_{X}$ :

$(*)^{\text {et }}$ Let $N \subseteq \Delta_{X}$ be any subgroup such that $H \subseteq N$ and $N / H$ is cyclic. Then there exists a surjection $N^{\text {ab }} \stackrel{\text { def }}{=} N /[N, N] \rightarrow Q$, where $Q$ is a free $\mathbf{Z}_{p}$-module of rank one, with the following properties: (i) there exists an open subgroup $\Gamma^{\prime} \subseteq \Gamma_{K}$ that stabilizes $N$ and $N^{\text {ab }} \rightarrow Q$ and acts trivially on $Q$; (ii) the surjection $N \rightarrow N / H$ factors through $Q$.

Then we have the following "group-theoretic" characterization of the quotient $\Delta_{X} \rightarrow \Delta_{X}^{\text {et }}$ :

Lemma 1.3. The kernel of $\Delta_{X} \rightarrow \Delta_{X}^{\text {et }}$ is the intersection of all open normal subgroups $H \subseteq \Delta_{X}$ such that $H$ satisfies the condition $(*)^{\text {et }}$.

Proof. The proof is entirely the same as that of Sections 3 and 8 of [Mzk1]. The basic idea is that if the covering corresponding to $\Delta_{X} / H$ is not étale (over $\mathcal{O}_{K}$ ), then it has nontrivial inertia subgroups. Such inertia subgroups have nontrivial cyclic subgroups. If we then apply $(*)^{\text {et }}$ to the case where $N / H$ is one of these nontrivial cyclic subgroups, then we have a contradiction, since the quotient $N \rightarrow N / H$ factors through the quotient $N \rightarrow Q$; moreover, the quotient $N \rightarrow Q$ necessarily corresponds to a covering which is étale over $\mathcal{O}_{K}$ because of the assumption concerning the action of $\Gamma^{\prime} \subseteq \Gamma_{K}$ on $Q$.

Let us review what we have done so far. So far, we have:

(1) constructed quotients $\Pi_{X_{K}} \rightarrow \Pi_{X_{K}}^{\mathrm{et}}$ and $\Delta_{X} \rightarrow \Delta_{X}^{\mathrm{et}}$, as well as a section $\Gamma_{K} \rightarrow \Pi_{X_{K}}^{\text {et }}$ via various geometric considerations concerning $X_{K}$;

(2) shown (when $g \geq 2$ ) that the above quotients and section may be reconstructed entirely "group-theoretically."

Here, we pause to make the following

Remark Concerning the Term "Group-Theoretic." In [Mzk1] and [Mzk2], we imparted mathematical rigor to the term "group-theoretic" (cf. the remark on this issue in Section 14 of [Mzk2]) by specifying that it meant "preserved by isomorphism." In the present paper, however, we would like to consider homomorphisms which are not necessarily isomorphisms. Thus, in the present paper, when we wish to state that a certain property or object is preserved by such homomorphisms, we shall state this explicitly without using 
the term "group-theoretic." In the above discussion, however, we recommend the reader to simply accept this term at the level of "common sense," since we will not use the "grouptheoreticity" stated in any of the results of this Section in the proof of any of the main theorems of this paper.

Finally, before continuing, we make the following important observation: Let $\alpha_{x}$ : $\Gamma_{K} \rightarrow \Pi_{X_{K}}$ denote the section of $\Pi_{X_{K}} \rightarrow \Gamma_{K}$ determined (up to composition with an inner automorphism induced by an element of $\left.\Delta_{X}\right)$ by a point $x \in X_{K}(K)$. Denote by $\alpha_{x}^{\text {et }}: \Gamma_{K} \rightarrow \Pi_{X_{K}}^{\mathrm{et}}$ the composite of $\alpha_{x}$ with $\Pi_{X_{K}} \rightarrow \Pi_{X_{K}}^{\mathrm{et}}$.

Lemma 1.4. We have $\alpha_{x}^{\mathrm{et}}=\theta_{X}$.

Proof. It suffices to show that the composite of $\alpha_{x}^{\text {et }}$ with the surjection $\zeta_{X}: \Pi_{X_{K}}^{\text {et }} \rightarrow$ $\Delta_{X}^{\text {et }}$ is trivial. Interpreted geometrically, this simply means that "the pull-back of any étale covering $Y \rightarrow X$ (i.e., étale over $\left.\mathcal{O}_{K}\right)$ to $\operatorname{Spec}(K)$ via $x$ is the trivial étale covering of $\operatorname{Spec}(K)$." But this assertion follows immediately from the fact that $\mathcal{O}_{K}$ is strictly henselian.

\section{Section 2: Review of Galois Cohomology}

Let $K$ be a p-adic field whose residue field is perfect. Let $S \rightarrow \operatorname{Spec}\left(\mathcal{O}_{K}\right)$ be a geometrically connected smooth morphism, and let $D \subseteq S$ be a relative (over $\mathcal{O}_{K}$ ) divisor with normal crossings. Let us write $S^{\log }$ for the $\log$ scheme obtained by equipping $S$ with the log structure defined by $D$ (as in [Kato]). Let us also assume that $S$ is small (in the sense of [Falt1], [Falt2]): Recall that this simply means that $S$ is affine, say, equal to $\operatorname{Spec}(R)$, and, moreover, étale over some $\mathcal{O}_{K}\left[X_{1}, \ldots, X_{d}\right]$ in such a way that $D$ is schematically the inverse image of the zero locus of the function $X_{1} \cdot \ldots \cdot X_{d}$. The reason we wish to deal with $\operatorname{small}(S, D)$ is that in [Falt1], certain Galois cohomology groups associated to such $(S, D)$ are computed explicitly by means of the theory of almost étale extensions. The purpose of this Section is to review certain consequences of the theory of [Falt1] and [Falt2] that are of relevance to us in this paper.

Let $R_{K} \stackrel{\text { def }}{=} R \otimes_{\mathcal{O}_{K}} K$. Let $R_{K} \rightarrow \bar{R}_{K}$ be the maximal extension of $R_{K}$ which is étale outside of $D_{K}$. Let $\bar{R}$ be the normalization of $R$ in $\bar{R}_{K}$. Let $\widehat{\bar{R}}$ (respectively, $\widehat{R}$ ) be the p-adic completion of $\bar{R}$ (respectively, $R$ ); let $\widehat{\bar{R}}_{K} \stackrel{\text { def }}{=} \widehat{\bar{R}} \otimes_{\mathcal{O}_{K}} K, \widehat{R}_{K} \stackrel{\text { def }}{=} \widehat{R} \otimes_{\mathcal{O}_{K}} K$. Let $\widehat{R}_{\mathcal{O}_{\bar{K}}}$ be the $p$-adic completion of $R \otimes_{\mathcal{O}_{K}} \mathcal{O}_{\bar{K}} ; \widehat{R}_{\bar{K}} \stackrel{\text { def }}{=}\left(\widehat{R}_{\mathcal{O}_{\bar{K}}}\right) \otimes_{\mathcal{O}_{K}} K$. Let $\Gamma_{R} \stackrel{\text { def }}{=} \operatorname{Gal}\left(\bar{R}_{K} / R_{K}\right)$. Thus, we have a natural surjection $\Gamma_{R} \rightarrow \Gamma_{K}$ whose kernel we denote by $\Delta_{R}^{\text {prf }}$. This gives us a natural exact sequence:

$$
1 \rightarrow \Delta_{R}^{\mathrm{prf}} \rightarrow \Gamma_{R} \rightarrow \Gamma_{K} \rightarrow 1
$$


Now we would like to compute some Galois cohomology groups:

Lemma 2.1. For all $j \in \mathbf{Z}$, we have:

(1) $H^{0}\left(\Delta_{R}^{\mathrm{prf}}, \widehat{\bar{R}}_{K}(j)\right)=\widehat{R}_{\bar{K}}(j)$;

(2) $H^{1}\left(\Delta_{R}^{\operatorname{prf}}, \widehat{\bar{R}}_{K}(j)\right)=\Omega_{S^{\log } / \mathcal{O}_{K}} \otimes_{R} \widehat{R}_{\bar{K}}(j-1)$.

(Here the " $(j)$ " is a Tate twist.)

Proof. This follows from Theorem 4.4 of Section I of [Falt1]. Indeed, (1) follows from [Falt1], Section I, Theorem 4.4, (i) (applied to the case $i=0$, and tensored with $K(j)$ ), while (2) follows from [Falt1], Section I, Theorem 4.4, (iv) (tensored with $K(j)$ ).

Lemma 2.2. We have: (i) $H^{0}\left(\Gamma_{K}, \widehat{R}_{\bar{K}}\right)=\widehat{R}_{K}$; (ii) $H^{n}\left(\Gamma_{K}, \widehat{R}_{\bar{K}}(j)\right)=0$ (for $j \neq 0$; $n=0,1)$; $\left(\right.$ iii) $H^{1}\left(\Gamma_{K}, \widehat{R}_{\bar{K}}\right)=\widehat{R}_{K}$.

Proof. This result is due to [Tate] in the case $R=\mathcal{O}_{K}$. (Indeed, in this case, (i) and (iii) follow from [Tate], §3.3, Theorem 1; while (ii) - at least in the case $j=1$ (the proof for arbitrary $j \neq 0$ is entirely similar) - follows from [Tate], §3.3, Theorem 2.) The slightly more general result stated here (i.e., for $R$ not necessarily equal to $\mathcal{O}_{K}$ ) follows by the same argument as that employed by Tate in the case $R=\mathcal{O}_{K}$ (the point being that in general, $R$ is $\mathcal{O}_{K}$-flat). Alternatively, the cohomology groups in the Lemma can also be computed using almost étale extensions as in [Falt1].

Lemma 2.3. For $n=0,1$, we have: (i) $H^{n}\left(\Gamma_{R}, \widehat{\bar{R}}_{K}\right)=\widehat{R}_{K} ;(i i) H^{n}\left(\Gamma_{R}, \widehat{\bar{R}}_{K}(-1)\right)=0$.

Proof. This follows from the Leray-Serre spectral sequence (applied to the exact sequence of groups that appears directly before Lemma 2.1), plus the preceding two Lemmas.

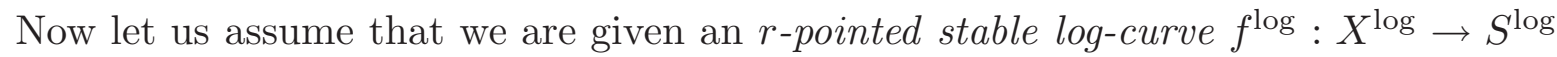
of genus $g$, where $2 g-2+r \geq 1$. (By this we mean that $X^{\log }$ is obtained by pulling back the universal log-curve $\mathcal{C}^{\log } \rightarrow \overline{\mathcal{M}}_{g, r}^{\log }$ via some $\log$ morphism $S^{\log } \rightarrow \overline{\mathcal{M}}_{g, r}^{\log }$. The $\log$ structure of the universal log curve is defined by the divisor with normal crossings which is the union of the marked points and the singular fibers. See, e.g., [Mzk3], Section 3, for more details; cf. also the Remark preceding Definition 1.1 of this paper for a review of the notion of a "pointed stable curve.") Let $U \stackrel{\text { def }}{=} S-D$. Then taking the relative first cohomology module of $\left.f\right|_{U_{K}}$ in the étale topology with coefficients in $\mathbf{Z}_{p}$ gives rise to a local system over $U_{K}$, hence a $\Gamma_{R}$-module $H^{\vee}$. As a $\mathbf{Z}_{p}$-module, $H^{\vee}$ is free of rank $=2 g$ 
(if $r=0$ ) and $=2 g+r-1$ (if $r>0$ ). Let $H$ be the $\Gamma_{R}$-module given by $\operatorname{Hom}_{\mathbf{Z}_{p}}\left(H^{\vee}, \mathbf{Z}_{p}\right)$. Now let

$$
\mathcal{H}_{0} \stackrel{\text { def }}{=}\left(\mathbf{R}^{1} f_{*} \mathcal{O}_{X}\right)^{\vee}
$$

(a vector bundle over $S$, or alternatively, a projective $R$-module, of rank $g$ ) and

$$
\mathcal{H}_{1} \stackrel{\text { def }}{=}\left(f_{*} \omega_{X} \log / S^{\log }\right)^{\vee}
$$

(a vector bundle over $S$, or alternatively, a projective $R$-module, whose rank is $=g$ if $r=0$ and $=g+r-1$ if $r>0)$.

Proposition 2.4. $\quad$ There is a natural exact sequence

$$
0 \rightarrow \mathcal{H}_{1} \otimes_{R} \widehat{\bar{R}}_{K}(1) \rightarrow H \otimes_{\mathbf{z}_{p}} \widehat{\bar{R}}_{K} \rightarrow \mathcal{H}_{0} \otimes_{R} \widehat{\bar{R}}_{K} \rightarrow 0
$$

which is compatible with the natural action of $\Gamma_{R}$ on all three terms.

Proof. This is an immediate consequence of the "Comparison Theorem" (Theorem 6.2 of [Falt2]). The validity of the proof of this Theorem given in [Falt2] has been disputed by various mathematicians. However, since Proposition 2.4 is a relatively weak consequence of the "Comparison Theorem," it already follows from the portion of [Falt2] that is not in dispute. (That is to say, we need only that $M$ is "Hodge-Tate," not that it is "crystalline.") Alternatively, although the sort of parametrized (i.e., over a base $S$ ) Hodge-Tate decomposition that we need here is not stated in [Falt1], it follows immediately from the theory of [Falt1] by exactly the same proof as that of the main result of [Falt1]. Yet another proof of this sort of result is given in [Hyodo] (the final Theorem - i.e., the "relative version" - in [Hyodo], §0.3), although here we need the (relatively straightforward) logarithmic generalization of [Hyodo]'s result.

\section{Section 3: The Weight Zero Quotient}

In this Section, we maintain the notation of the preceding Section. The purpose of this Section is to give a (rather weak) nonabelian analogue of Proposition 2.4. In fact, a much stronger "nonabelian comparison theorem" for the fundamental group of a curve can be proven, but since I do not know of any place where such a result has been written up, and, moreover, in this paper, only a relatively weak nonabelian comparison theorem is needed, I decided instead to give a rather short ad hoc treatment of this issue which will, nonetheless, be sufficient for the purposes of this paper. 
Let us denote the fundamental group of $X_{K}^{\log }$ by $\Pi_{X_{K}}^{\text {prf }}$. Thus, we have a natural surjection $\Pi_{X_{K}}^{\mathrm{prf}} \rightarrow \Gamma_{R}$. As usual, we denote the kernel of this surjection by $\Delta_{X}^{\mathrm{prf}} \subseteq \Pi_{X_{K}}^{\mathrm{prr}}$, and the maximal pro- $p$ quotient of $\Delta_{X}^{\text {prf }}$ by $\Delta_{X}$. Moreover, by forming the quotient of $\Pi_{X_{K}}^{\text {prf }}$ by the kernel of $\Delta_{X}^{\text {prf }} \rightarrow \Delta_{X}$, we obtain $\Pi_{X_{K}}$. Thus, just as in Section 1 , we have an exact sequence

$$
1 \rightarrow \Delta_{X} \rightarrow \Pi_{X_{K}} \rightarrow \Gamma_{R} \rightarrow 1
$$

Moreover, any section $\sigma: S \rightarrow X$ of $X \rightarrow S$ whose image avoids the marked points and nodes defines a section $\pi_{\sigma}: \Gamma_{R} \rightarrow \Pi_{X_{K}}$ of the above exact sequence. Such a section $\pi_{\sigma}$ then defines an action of $\Gamma_{R}$ on $\Delta_{X}$ by conjugation. Note that until one specifies the section $\alpha_{\sigma}: \Gamma_{R} \rightarrow \Pi_{X_{K}}$, one only has an outer action of $\Gamma_{R}$ on $\Delta_{X}$ (i.e., an action defined only up to inner automorphisms); that is to say, in general, there is no natural action (in the usual, non-outer sense) of $\Gamma_{R}$ on $\Delta_{X}$ until one specifies a section of $\Pi_{X_{K}} \rightarrow \Gamma_{R}$.

The next step is to introduce the Malčev completion of $\Delta_{X}$. We refer to [Del], [NT] for more details. In fact, for our purposes, it will be sufficient to consider a truncated form of the Malčev completion of $\Delta_{X}$. This truncated form may be defined as follows: Let $\mathcal{C}$ be the category of finite dimensional $\mathbf{Q}_{p}$-vector spaces $V$ equipped with a continuous $\Delta_{X}$-action that factors through $\Delta_{X} /\left[\Delta_{X},\left[\Delta_{X}, \Delta_{X}\right]\right]$ and which admits a $\Delta_{X}$-invariant filtration on whose subquotients $\Delta_{X}$ acts trivially. (The morphisms of this category are the $\mathbf{Q}_{p}\left[\Delta_{X}\right]$ linear morphisms $V \rightarrow V^{\prime}$.) Then $\mathcal{C}$ is a Tannakian category over $\mathbf{Q}_{p}$, hence gives rise to an algebraic group $M_{X}$ over $\mathbf{Q}_{p}$. Moreover, this algebraic group $M_{X}$ is unipotent, hence corresponds to a nilpotent Lie algebra $\mathfrak{M}_{X}$. For any $\mathbf{Q}_{p}$-algebra $A$, we shall write $\left(M_{X}\right)_{A}$ (respectively, $\left(\mathfrak{M}_{X}\right)_{A}$ ) for $M_{X} \otimes_{\mathbf{Q}_{p}} A$ (respectively, $\mathfrak{M}_{X} \otimes_{\mathbf{Q}_{p}} A$ ).

Let us write $\mathfrak{M}_{X}[1]$ for the commutator $\left[\mathfrak{M}_{X}, \mathfrak{M}_{X}\right]$ of this Lie algebra, and $\mathfrak{M}_{X}[0]$ for the quotient $\mathfrak{M}_{X} / \mathfrak{M}_{X}[1]$. Then $\mathfrak{M}_{X}[0]$ may be identified with $H_{\mathbf{Q}_{p}} \stackrel{\text { def }}{=} H \otimes \mathbf{z}_{p} \mathbf{Q}_{p}$ (where $H$ is as in the discussion following Lemma 2.3 in Section 2). Thus, we get an exact sequence

$$
0 \rightarrow \mathfrak{M}_{X}[1] \rightarrow \mathfrak{M}_{X} \rightarrow \mathfrak{M}_{X}[0]=H_{\mathbf{Q}_{p}} \rightarrow 0
$$

Moreover, the commutator $[-,-]$ defines a surjection

$$
c_{X}: \wedge^{2} H_{\mathbf{Q}_{p}} \rightarrow \mathfrak{M}_{X}[1]
$$

Now observe that although there is no natural action of $\Gamma_{R}$ on $\mathfrak{M}_{X}$ (unless one chooses a section of $\Pi_{X_{K}} \rightarrow \Gamma_{R}$ ), there is nonetheless a natural action of $\Gamma_{R}$ on $\mathfrak{M}_{X}[0]$ and $\mathfrak{M}_{X}[1]$ with respect to which $c_{X}$ is equivariant. Now we have the following classical result

Lemma 3.1. The kernel of $c_{X}$ is zero if $r>0$. If $r=0$, then the kernel of $c_{X}$ is onedimensional, and equal to the image of the dual to the intersection form $\wedge^{2} H_{\mathbf{Q}_{p}}^{\vee} \rightarrow \mathbf{Q}_{p}(-1)$ (defined by the cup product pairing on the cohomology of $\Delta_{X}$ ). 
Proof. If $r>0$, then $\Delta_{X}$ is a free pro- $p$ group. Thus, in this case the result follows by consideration of the fact that a representation on a vector space $V$ of the free group on $n$ generators is the same as giving $n$ endomorphisms of $V$. If $r=0$, then $\Delta_{X}$ is the quotient of a free pro- $p$ group by a single relation; it is this relation which generates the kernel of $c_{X}$. Moreover, it is easy to see from the well-known explicit form of this relation that its image in $\wedge^{2} H_{\mathbf{Q}_{p}}$ is precisely as specified in the statement of the Lemma (cf. the discussion in $\S 2$ of $[\mathrm{NT}])$.

Next, we would like to construct a certain special quotient $\mathfrak{Z}_{X}$ of $\left(\mathfrak{M}_{X}\right) \widehat{\bar{R}}_{K}$. By the well-known categorical equivalence between unipotent algebraic groups and nilpotent Lie algebras (cf. [Del], $\S 9$ ), this quotient will define a quotient $Z_{X}$ of $\left(M_{X}\right) \widehat{\bar{R}}_{K}$. The construction of $\mathfrak{Z}_{X}$ from $\left(\mathfrak{M}_{X}\right)_{\widehat{\bar{R}}_{K}}$ consists of two steps. The first step is as follows: Consider the surjection

$$
\wedge^{2} H \otimes_{\mathbf{z}_{p}} \widehat{\bar{R}}_{K} \rightarrow \wedge^{2} \mathcal{H}_{0} \otimes_{R} \widehat{\bar{R}}_{K}
$$

defined by projecting by means of the surjection in the short exact sequence of Proposition 2.4. It follows from Lemmas 2.3 (ii) (in the case $n=0$ ) and 3.1 that this surjection factors through $\left(\mathfrak{M}_{X}[1]\right)_{\widehat{\bar{R}}_{K}}$. Thus, we obtain a surjection

$$
\left(\mathfrak{M}_{X}[1]\right)_{\widehat{\bar{R}}_{K}} \rightarrow \wedge^{2} \mathcal{H}_{0} \otimes_{R} \widehat{\bar{R}}_{K}
$$

By pushing forward the exact sequence $0 \rightarrow \mathfrak{M}_{X}[1] \rightarrow \mathfrak{M}_{X} \rightarrow \mathfrak{M}_{X}[0] \rightarrow 0$ (tensored over $\mathbf{Q}_{p}$ with $\widehat{\bar{R}}_{K}$ ) via this surjection, we thus obtain a Lie algebra $\mathfrak{U}_{X}$ (over $\widehat{\bar{R}}_{K}$ ). Thus, we have a surjection of Lie algebras $\left(\mathfrak{M}_{X}\right)_{\widehat{\bar{R}}_{K}} \rightarrow \mathfrak{U}_{X}$, together with an exact sequence

$$
0 \rightarrow \mathfrak{U}_{X}[1] \rightarrow \mathfrak{U}_{X} \rightarrow \mathfrak{U}_{X}[0] \rightarrow 0
$$

where $\mathfrak{U}_{X}[1] \stackrel{\text { def }}{=}\left[\mathfrak{U}_{X}, \mathfrak{U}_{X}\right]=\wedge^{2} \mathcal{H}_{0} \otimes_{R} \widehat{\bar{R}}_{K}$, and $\mathfrak{U}_{X}[0]=\left(\mathfrak{M}_{X}[0]\right)_{\widehat{R}_{K}}$.

Now we come to the second step in the construction of $\mathfrak{Z}_{X}$. First let us denote by $\mathfrak{U}_{X}[0] \rightarrow \mathfrak{U}_{X}[0,0] \stackrel{\text { def }}{=} \mathcal{H}_{0} \otimes_{R} \widehat{\bar{R}}_{K}$ the surjection defined by the surjection in the short exact sequence of Proposition 2.4. Let $\mathfrak{U}_{X}[0,1] \subseteq \mathfrak{U}_{X}[0]$ be the kernel of this surjection. Let $\mathfrak{B}_{X} \subseteq \mathfrak{U}_{X}$ denote the inverse image of $\mathfrak{U}_{X}[0,1] \subseteq \mathfrak{U}_{X}[0]$ under the surjection $\mathfrak{U}_{X} \rightarrow \mathfrak{U}_{X}[0]$. Then it follows from the definition of $\mathfrak{U}_{X}$ that $\mathfrak{B}_{X}$ is an abelian Lie algebra which, in fact, lies in the center of the Lie algebra of $\mathfrak{U}_{X}$. (Indeed, this will follow as soon as we show the vanishing of $\left[\mathfrak{B}_{X}, \mathfrak{U}_{X}\right]$, which is equal to the image of $\mathfrak{U}_{X}[0,1] \wedge \mathfrak{U}_{X}[0] \subseteq \wedge^{2} H_{\mathbf{Q}_{p}}$ under the composite of $c_{X}: \wedge^{2} H_{\mathbf{Q}_{p}} \rightarrow \mathfrak{M}_{X}[1]$ with the projection $\mathfrak{M}_{X}[1] \rightarrow \mathfrak{U}_{X}[1]=\wedge^{2} \mathcal{H}_{0} \otimes_{R} \widehat{\bar{R}}_{K}$; but this image is zero, by the definition of $\mathfrak{U}_{X}[0,1]$.) This observation implies, in particular, that although, a priori, we have only an outer action of $\Gamma_{R}$ on $\mathfrak{U}_{X}$, hence on $\mathfrak{B}_{X}$, in fact, we get a natural (non-outer) action of $\Gamma_{R}$ on $\mathfrak{B}_{X}$. (That is, the point is that the various 
actions of $\Gamma_{R}$ on $\mathfrak{B}_{X}$ arising from different sections of $\Pi_{X_{K}} \rightarrow \Gamma_{R}$ differ only by an automorphism on $\mathfrak{B}_{X}$ induced by conjugation by some element of $\Delta_{X}$; but since $\mathfrak{B}_{X}$ lies in the center of $\mathfrak{U}_{X}$, it follows that such an automorphism of $\mathfrak{B}_{X}$ is always equal to the identity.)

Next, let us note that we have exact sequences

$$
0 \rightarrow \mathfrak{B}_{X} \rightarrow \mathfrak{U}_{X} \rightarrow \mathfrak{U}_{X}[0,0] \rightarrow 0
$$

and

$$
0 \rightarrow \mathfrak{U}_{X}[1] \rightarrow \mathfrak{B}_{X} \rightarrow \mathfrak{U}_{X}[0,1] \rightarrow 0
$$

where the latter exact sequence is an exact sequence of $\Gamma_{R}$-modules (a statement which has meaning as a result of the observation at the end of the preceding paragraph). Now observe that Lemma 2.3 (ii) (for $n=0,1$ - note that here we use that $\mathfrak{U}_{X}[1]=\wedge^{2} \mathcal{H}_{0} \otimes_{R} \widehat{\bar{R}}_{K}$ is "of weight zero," while $\mathfrak{U}_{X}[0,1]=\mathcal{H}_{1} \otimes_{R} \widehat{\bar{R}}_{K}(1)$ is "of weight one") implies that the latter exact sequence admits a unique $\Gamma_{R}$-equivariant splitting: $\mathfrak{B}_{X} \rightarrow \mathfrak{U}_{X}[1]$. Moreover, since $\mathfrak{B}_{X}$ is (as observed above) contained in the center of the Lie algebra $\mathfrak{U}_{X}$, it follows that the kernel of this splitting $\mathfrak{B}_{X} \rightarrow \mathfrak{U}_{X}[1]$ forms a Lie ideal in $\mathfrak{U}_{X}$. Thus, if we then push forward the former exact sequence via this surjection $\mathfrak{B}_{X} \rightarrow \mathfrak{U}_{X}[1]$, we obtain a Lie algebra $\mathfrak{Z}_{X}$. As usual, this Lie algebra fits into an exact sequence

$$
0 \rightarrow \mathfrak{Z}_{X}[1] \rightarrow \mathfrak{Z}_{X} \rightarrow \mathfrak{Z}_{X}[0] \rightarrow 0
$$

Moreover, one has natural identifications: $\mathfrak{Z}_{X}[1]=\wedge^{2} \mathfrak{Z}_{X}[0]$ (via the commutator map); and $\mathfrak{Z}_{X}[0]=\mathcal{H}_{0} \otimes_{R} \widehat{\bar{R}}_{K}$ (induced by the surjection of the short exact sequence of Proposition 2.4). In particular, $\mathfrak{Z}_{X}[0]$ is of rank $g$ over $\widehat{\bar{R}}_{K}$. Finally, as noted above, $\mathfrak{Z}_{X}$ defines a unipotent algebraic group $Z_{X}$.

Definition 3.2. $\quad$ We shall refer to $Z_{X}$ as the weight zero quotient of $\Delta_{X}$ (even though it is not literally a quotient). (Here, the " $Z$ " of $Z_{X}$ stands for the "zero" of "weight zero quotient.")

Now let us fix a (continuous) section

$$
\alpha: \Gamma_{R} \rightarrow \Pi_{X_{K}}
$$

Then $\alpha$ induces an action of $\Gamma_{R}$ on $\Delta_{X}$. Since $\mathfrak{Z}_{X}$ was formed naturally - and, for that matter, group-theoretically - from $\Delta_{X}$, we thus obtain an action of $\Gamma_{R}$ on $\mathfrak{Z}_{X}$ which, in 
general, will depend on the choice of $\alpha$. The next issue we would like to consider is to what extent the sequence of $\Gamma_{R}$-modules

$$
0 \rightarrow \mathfrak{Z}_{X}[1] \rightarrow \mathfrak{Z}_{X} \rightarrow \mathfrak{Z}_{X}[0] \rightarrow 0
$$

admits a $\Gamma_{R}$-equivariant section. At any rate, this exact sequence defines an extension class

$$
\eta_{\alpha} \in H^{1}\left(\Gamma_{R},\left(\mathfrak{Z}_{X}[0]\right)^{\vee} \otimes_{\widehat{\bar{R}}_{K}} \mathfrak{Z}_{X}[1]\right)
$$

By Lemma 2.3 (i) (for $n=1$ - here we use that both $\mathfrak{Z}_{X}[0]=\mathcal{H}_{0} \otimes_{R} \widehat{\bar{R}}_{K}$ and $\mathfrak{Z}_{X}[1]=$ $\wedge^{2} \mathfrak{Z}_{X}[0]$ are "of weight zero"), it follows that this Galois cohomology group may be identified naturally with

$$
\left(\mathcal{H}_{0}^{\vee} \otimes_{R} \wedge^{2} \mathcal{H}_{0}\right) \otimes_{R} \widehat{R}_{K}
$$

That is to say, one may think of $\eta_{\alpha}$ as a section of a certain vector bundle over $\widehat{R}_{K}$.

Proposition 3.3. Suppose that $\alpha$ arises as the $\alpha_{\sigma}$ associated to some section $\sigma: S \rightarrow X$ (whose image avoids the marked points and nodes - cf. the discussion at the beginning of this Section). Then $\eta_{\alpha}=0$.

Proof. Since everything is functorial, one reduces immediately to the universal case, as follows: In the present context, the essential data that we begin with is a $r$-pointed stable curve of genus $g$ (i.e., $f^{\log }: X^{\log } \rightarrow S^{\log }$ ), plus a section (i.e., $\sigma$ ). The moduli stack for this data is (a certain dense open substack of) the tautological curve $\mathcal{C} \rightarrow \overline{\mathcal{M}}_{g, r}$ over the moduli stack $\overline{\mathcal{M}}_{g, r}$ of $r$-pointed stable curves of genus $g$ over $\mathbf{Z}_{p}$. Thus, by "restriction," it suffices to prove the Proposition in the case where $S$ is étale over the algebraic stack $\mathcal{C}$. Also, since everything involved commutes with base-extension, it is easy to see that we may assume that $k$ is algebraically closed. Then over some dense open $T \subseteq(S-D) \otimes \mathcal{O}_{K} k$, the Jacobian of $X \otimes \mathcal{O}_{K} k$ will be ordinary. Thus, every point of $\beta \in S\left(\mathcal{O}_{K}\right)$ that maps $\operatorname{Spec}(k) \subseteq \operatorname{Spec}\left(\mathcal{O}_{K}\right)$ into $T$ defines an ordinary (in the sense of Definition 1.1) hyperbolic curve $Y_{\beta} \rightarrow \operatorname{Spec}(K)$ over $K$ (by restricting (the complement of the marking sections in) $X \rightarrow S$ to $\beta)$.

Next, observe that that if we restrict the section $\sigma$ to the point $\beta$, we get a section $\sigma_{\beta}: \operatorname{Spec}(K) \rightarrow Y_{\beta}$ which induces an action of $\Gamma_{K}$ on $\Delta_{Y_{\beta}}^{\text {et }}$ (i.e., the quotient $\Delta_{Y_{\beta}} \rightarrow \Delta_{Y_{\beta}}^{\text {et }}$ considered in Section 1). By Lemmas 1.2 and 1.4, this action is, in fact, the trivial action. Now I claim that in the case of such an ordinary curve:

The quotient $\Delta_{Y_{\beta}} \rightarrow \Delta_{Y_{\beta}}^{\text {et }}$ (considered in Section 1) induces a natural (in particular, $\Gamma_{K}$-equivariant) isomorphism of the weight zero quotient 
$Z_{Y_{\beta}}$ of $\Delta_{Y_{\beta}}\left(=\Delta_{X}\right)$ with the Malčev completion of $\Delta_{Y_{\beta}}^{\text {et }}$ (truncated at the second step and tensored over $\mathbf{Z}_{p}$ with $\widehat{\bar{K}}$ ).

Indeed, it follows immediately from the construction of $Z_{Y_{\beta}}$ that the surjection $\Delta_{Y_{\beta}} \rightarrow \Delta_{Y_{\beta}}^{\text {et }}$ induces a morphism from $Z_{Y_{\beta}}$ to the "weight zero quotient" (i.e., constructed in a fashion analogous to the construction of $Z_{Y_{\beta}}$ ) of $\Delta_{Y_{\beta}}^{\mathrm{et}}$. On the other hand, since the action of $\Gamma_{K}$ on $\Delta_{Y_{\beta}}^{\mathrm{et}}$ is trivial, it follows that the "weight zero quotient" of $\Delta_{Y_{\beta}}^{\mathrm{et}}$ is just the Malčev completion of $\Delta_{Y_{\beta}}^{\text {et }}$ (truncated at the second step and tensored over $\mathbf{Z}_{p}$ with $\widehat{\bar{K}}$ ). Moreover, it is clear that the map induced on abelianizations by this morphism from $Z_{Y_{\beta}}$ to this weight zero quotient of $\Delta_{Y_{\beta}}^{\text {et }}$ is an isomorphism. (Indeed, surjectivity follows from the fact that we are dealing (by definition) with various quotients of a single object; injectivity then follows from Proposition 2.4 and Lemma 2.3 (ii) (for $n=0$ ), and the fact that both abelianizations are of rank $g$ over $\widehat{\bar{K}}$.) Thus, (since $\Delta_{Y_{\beta}}^{\text {et }}$ is a free pro- $p$ group of rank $g$ - cf. the discussion following Definition 1.1) we conclude that we get an isomorphism as stated in the "claim." This completes the verification of the claim.

Thus, any minimal choice of generators of $\Delta_{Y_{\beta}}^{\text {et }}$ (which will necessarily be fixed by $\Gamma_{K}$ ) defines a splitting of the sequence of $\Gamma_{K}$-modules

$$
0 \rightarrow \mathfrak{Z}_{X}[1] \rightarrow \mathfrak{Z}_{X} \rightarrow \mathfrak{Z}_{X}[0] \rightarrow 0
$$

Here the $\Gamma_{K}$-action is given by composing the $\Gamma_{R}$-action considered above with the morphism - well-defined up to composition with an inner automorphism (which does not bother us since this inner automorphism corresponds to a coboundary in the computation of the cohomology class $\eta_{\alpha}$ that we are interested in) $-\Gamma_{K} \rightarrow \Gamma_{R}$ defined by $\beta$. But from the definition of the extension class $\eta_{\alpha}$, this means that the restriction

$$
\left.\eta_{\alpha}\right|_{\beta} \in\left(\mathcal{H}_{0}^{\vee} \otimes_{R} \wedge^{2} \mathcal{H}_{0}\right) \otimes_{R, \beta} K
$$

of $\eta_{\alpha}$ to the point $\beta$ is zero. On the other hand, if $\eta_{\alpha}$ is zero when restricted to any such $\beta$ (i.e., any $\beta \in S\left(\mathcal{O}_{K}\right)$ that $\operatorname{maps} \operatorname{Spec}(k) \subseteq \operatorname{Spec}\left(\mathcal{O}_{K}\right)$ into $\left.T\right)$, it is clear that $\eta_{\alpha}$ itself must be zero. This completes the proof of the Proposition.

Note that so far, in this Section and the last, we have been dealing with families of curves, parametrized over a base $S$. Before continuing on to the next Section, it is worthwhile to go back to the case of "a single curve" over $K$ in order to make explicit the consequences for such single curves of the theory developed thus far.

Thus, let $X_{K} \rightarrow \operatorname{Spec}(K)$ be a hyperbolic curve. As in Section 1, we have an exact sequence

$$
1 \rightarrow \Delta_{X} \rightarrow \Pi_{X_{K}} \rightarrow \Gamma_{K} \rightarrow 1
$$


Then let us first note that the theory reviewed in Section 2 can be applied in the present nonparametrized situation as well to produce (following precisely the same recipe as in the parametrized situation considered as above) a Lie algebra $\mathfrak{Z}_{X}$, together with a group $Z_{X}$ (both over $\widehat{\bar{K}}$ ).

Definition 3.4. We shall refer to $Z_{X}$ as the weight zero quotient of $\Delta_{X}$.

Let $x \in X_{K}(K)$. Then $x$ defines a section $\alpha_{x}: \Gamma_{K} \rightarrow \Pi_{X_{K}}$ (well-defined up to composition with an inner automorphism arising from $\Delta_{X}$ ). Moreover, $\alpha_{x}$ defines an action of $\Gamma_{K}$ on $\mathfrak{Z}_{X}$.

Proposition 3.5. Relative to the action of $\Gamma_{K}$ on $\mathfrak{Z}_{X}$ defined by $\alpha_{x}$, the exact sequence of $\Gamma_{K}$-modules

$$
0 \rightarrow \mathfrak{Z}_{X}[1] \rightarrow \mathfrak{Z}_{X} \rightarrow \mathfrak{Z}_{X}[0] \rightarrow 0
$$

splits.

Proof. Note that this Proposition is not, strictly speaking, a special case of Proposition 3.3 (i.e., where we take $S=\mathcal{O}_{K}$ ), since Proposition 3.3 only addresses the case where the divisor of bad reduction is flat over $\mathcal{O}_{K}$. However, by means of specialization, one can reduce the present Proposition to the "universal case" considered during the proof of Proposition 3.3. Moreover, in this case, Proposition 3.3 already tells us that the relevant exact sequence is split. This completes the proof.

Remark. Let us write $\left(\Delta_{X}\right)_{\mathbf{Q}_{p}}$ for the full (i.e., not truncated as above) Malčev completion (cf. [Del], [NT]) of $\Delta_{X}$. Then in some sense, Proposition 3.5 above is a truncated version of a theorem that states "relative to the action of $\Gamma_{K}$ on $\left(\Delta_{X}\right){ }_{\mathbf{Q}_{p}}$ defined by $\alpha_{x},\left(\Delta_{X}\right)_{\mathbf{Q}_{p}}$ is Hodge-Tate." Here, since $\left(\Delta_{X}\right)_{\mathbf{Q}_{p}}$ is an inverse limit of unipotent algebraic groups, one can interpret "Hodge-Tate" to mean that the Lie algebras of each of these unipotent algebraic groups are Hodge-Tate representations. In fact, it is not difficult to prove that $\left(\Delta_{X}\right)_{\mathbf{Q}_{p}}$ is Hodge-Tate (even for arbitrary higher-dimensional smooth $X_{K} \rightarrow \operatorname{Spec}(K)$ ) as follows: One reduces the higher-dimensional case to the curve case by cutting with hyperplane sections. Then for curves, by considering the universal case, one can reduce to the case of curves smooth over $\mathcal{O}_{K}$. Finally, for curves smooth over $\mathcal{O}_{K}$, one can apply the techniques of [Falt1] (by considering cohomology spaces with coefficients valued in unipotent algebraic groups over $\left.\mathbf{Q}_{p}\right)$. This shows that $\left(\Delta_{X}\right)_{\mathbf{Q}_{p}}$ is Hodge-Tate. Moreover, one can also construct a nontruncated "weight zero quotient $\left(\Delta_{X}\right)_{\mathbf{Q}_{p}} \rightarrow Z_{X}^{\infty}$ " (that is to say, if $X_{K}$ is a curve of genus $g$, then $Z_{X}^{\infty}$ will be ( $\Gamma_{K}$-equivariantly) isomorphic to the Malčev completion over $\widehat{\bar{K}}$ of the free group on $g$ generators). In fact, it is even possible to show that $\left(\Delta_{X}\right)_{\mathbf{Q}_{p}}$ is $d e$ Rham, but we shall not pursue such issues here since they are not relevant to the proof of 
the main results of this paper. Nevertheless, we remark that this sort of theorem has been verified by A. Shiho ([Shiho]), in a manuscript in preparation.

\section{Section 4: J-Geometric Sections}

We maintain the notations of the latter portion of Section 3. Moreover, we assume that our curve $X_{K} \rightarrow \operatorname{Spec}(K)$ is proper (hence of genus $\geq 2$ ) and that $X_{K}(K) \neq \emptyset$. In this Section, we would like to consider a continuous homomorphism

$$
\alpha: \Gamma_{K} \rightarrow \Pi_{X_{K}}
$$

which defines a section of the surjection $\Pi_{X_{K}} \rightarrow \Gamma_{K}$. The section $\alpha$ defines an action of $\Gamma_{K}$ on $\Delta_{X}$ by conjugation, and hence also an action of $\Gamma_{K}$ on $\mathfrak{Z}_{X}$. In particular, we would like to consider the significance of the following condition on $\alpha$ :

$(*)^{\text {spl }}$ The exact sequence of $\Gamma_{K}$-modules (relative to the action defined by $\alpha)$

$$
0 \rightarrow \mathfrak{Z}_{X}[1] \rightarrow \mathfrak{Z}_{X} \rightarrow \mathfrak{Z}_{X}[0] \rightarrow 0
$$

splits.

The main result of this Section is to show that the group-theoretic condition $(*)^{\mathrm{spl}}$ is equivalent to the statement that $\alpha$ is "J-geometric" (a term which means that $\alpha$ acts in some respects as if it came from a geometric point $x \in X_{K}(K)$ - see Definition 4.3 for a precise definition).

To do this, first we need to recall certain facts concerning Jacobians and their fundamental groups. For $d \in \mathbf{Z}$, let $J_{X}^{(d)} \rightarrow \operatorname{Spec}(K)$ be the Picard scheme of line bundles on $X_{K}$ of degree $d$. Thus, $J_{X}^{(d)}$ is a torsor over $J_{X}=J_{X}^{(0)}$, the Jacobian of $X_{K}$. Note that $J_{X}^{(d)}$ is defined even if $X_{K}$ does not admit any $K$-rational points, as the scheme representing the étale sheafification of the usual Picard functor of degree $d$ line bundles. Note that a basepoint $x \in X_{K}(\Omega)$ (where $\Omega$ is the algebraically closed field of Section 0 ) may be regarded as a degree one divisor on $X_{\Omega}=X_{K} \otimes_{K} \Omega$, hence (by multiplying this divisor by $d$ ) we obtain a point $x_{d} \in J_{X}^{(d)}(\Omega)$. This allows us to define the arithmetic fundamental group $\pi_{1}\left(J_{X}^{(d)}, x_{d}\right)$, as well as its geometric counterpart $\pi_{1}\left(J_{X_{\bar{K}}}^{(d)}, x_{d}\right)$. If we form the quotient of $\pi_{1}\left(J_{X}^{(d)}, x_{d}\right)$ by the kernel of $\pi_{1}\left(J_{X_{\bar{K}}}^{(d)}, x_{d}\right) \rightarrow \Delta_{J_{X}^{(d)}}$ (i.e., the projection to the maximal pro- $p$ quotient of the geometric fundamental group), then we obtain a topological group $\Pi_{J_{X}^{(d)}}$. Moreover, we have a natural exact sequence 


$$
1 \rightarrow \Delta_{J_{X}^{(d)}} \rightarrow \Pi_{J_{X}^{(d)}} \rightarrow \Gamma_{K} \rightarrow 1
$$

Finally, the natural embedding $X_{K} \hookrightarrow J_{X}^{(1)}$ induces a natural surjection $\Pi_{X_{K}} \rightarrow \Pi_{J_{X}^{(1)}}$, whose kernel is the commutator subgroup of $\Delta_{X}$.

Now let us observe that we can reconstruct $\Pi_{J_{X}^{(d)}}$ from $\Pi_{X_{K}} \rightarrow \Gamma_{K}$, as follows. First of all, we can reconstruct $\Pi_{J_{X}^{(1)}}$ as the quotient of $\Pi_{X_{K}}$ by the commutator subgroup of $\Delta_{X}$. Next, let us observe that, since (for all $d \in \mathbf{Z}$ ) $J_{X}^{(d)}$ is a $J_{X}$-torsor, considering the action of $J_{X}$ on $J_{X}^{(d)}$ allows one to identify $\Delta_{J_{X}^{(d)}}$ with $\Delta_{J_{X}}$. Thus, we have an exact sequence

$$
1 \rightarrow \Delta_{J_{X}} \rightarrow \Pi_{J_{X}^{(1)}} \rightarrow \Gamma_{K} \rightarrow 1
$$

If we consider (for nonzero $d$ ), the morphism $J_{X}^{(1)} \rightarrow J_{X}^{(d)}$ given by multiplication by $d$, we see that the result of pushing forward this sequence by means of the morphism $\Delta_{J_{X}} \stackrel{d}{\rightarrow} \Delta_{J_{X}}$ (i.e., multiplication by $d$ ) gives rise to an exact sequence which can be naturally identified with the exact sequence

$$
1 \rightarrow \Delta_{J_{X}^{(d)}}=\Delta_{J_{X}} \rightarrow \Pi_{J_{X}^{(d)}} \rightarrow \Gamma_{K} \rightarrow 1
$$

This completes our review of Jacobians and their fundamental groups.

Now recall from the definition of $\mathfrak{Z}_{X}[0]$ in Section 3 that $\mathfrak{Z}_{X}[0]$ may be identified with the weight zero portion of $\Delta_{J_{X}} \otimes_{\mathbf{z}_{p}} \widehat{\bar{K}}$. In particular, one has a natural $\Gamma_{K}$-equivariant morphism $\Delta_{J_{X}} \rightarrow \mathfrak{Z}_{X}[0]$. Thus, by pushing forward the exact sequence of the preceding paragraph (for $d=1$ ) by means of this morphism, we obtain a morphism of exact sequences of topological groups as follows:

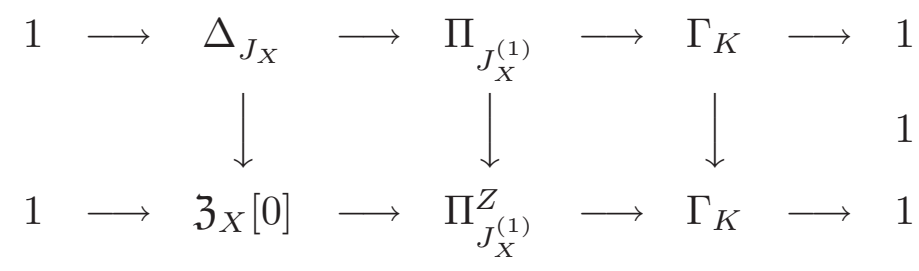

Next, we would like to consider sections of $\Pi_{J_{X}^{(1)}} \rightarrow \Gamma_{K}$ and $\Pi_{J_{X}^{(1)}}^{Z} \rightarrow \Gamma_{K}$. Recall that the difference between any two sections of $\Pi_{J_{X}^{(1)}} \rightarrow \Gamma_{K}$ (respectively, $\Pi_{J_{X}^{(1)}}^{Z} \rightarrow \Gamma_{K}$ ) is given by an element of $H^{1}\left(K, \Delta_{J_{X}}\right)$ (respectively, $\left.H^{1}\left(K, \mathfrak{Z}_{X}[0]\right)\right)$. Moreover, if $\theta_{\mathcal{L}}, \theta_{\mathcal{M}}: \Gamma \rightarrow \Pi_{J_{X}^{(1)}}$ are sections that arise from geometric points $\mathcal{L}, \mathcal{M} \in J_{X}^{(1)}(K)$, then the difference

$$
\delta \stackrel{\text { def }}{=} \theta_{\mathcal{L}}-\theta_{\mathcal{M}} \in H^{1}\left(K, \Delta_{J_{X}}\right)
$$


maps to zero in $H^{1}\left(K, \mathfrak{Z}_{X}[0]\right)$ ([BK], Example 3.11 - cf. the proof of Lemma 4.1 for some more details). Thus, it follows that the section $\theta_{\mathcal{L}}[0]: \Gamma_{K} \rightarrow \Pi_{J_{X}^{(1)}}^{Z}$ obtained from $\theta_{\mathcal{L}}$ by composing $\theta_{\mathcal{L}}$ with $\Pi_{J_{X}^{(1)}} \rightarrow \Pi_{J_{X}^{(1)}}^{Z}$ is independent (up to composition with an inner automorphism of $\Pi_{J_{X}^{(1)}}^{Z}$ arising from $\left.\mathfrak{Z}_{X}[0] \subseteq \Pi_{J_{X}^{(1)}}^{Z}\right)$ of the choice of $\mathcal{L}$. In particular, as long as $J_{X}^{(1)}(K)$ is nonempty (which is the case here, since $X_{K}(K)$ has been assumed to be nonempty), we thus obtain a canonical section (well-defined up to composition with an inner automorphism of $\Pi_{J_{X}^{(1)}}^{Z}$ arising from $\left.\mathfrak{Z}_{X}[0] \subseteq \Pi_{J_{X}^{(1)}}^{Z}\right)$

$$
\theta_{\text {geom }}: \Gamma_{K} \rightarrow \Pi_{J_{X}^{(1)}}^{Z}
$$

of $\Pi_{J_{X}^{(1)}}^{Z} \rightarrow \Gamma_{K}$

Now let $\theta: \Gamma_{K} \rightarrow \Pi_{J_{X}^{(1)}}$ be an arbitrary section, and let $\theta[0]: \Gamma_{K} \rightarrow \Pi_{J_{X}^{(1)}}^{Z}$ denote the section induced by $\theta$. Then taking the difference between $\theta[0]$ and $\theta_{\text {geom }}$ defines a cohomology class

$$
\delta_{\theta} \in H^{1}\left(K, \mathfrak{Z}_{X}[0]\right)=\mathcal{H}_{0}
$$

(where the last equality follows from Lemma 2.3, applied in the case $R_{K}=K$ ). Now we have the following result:

Lemma 4.1. Suppose that the residue field $k$ is finite. Then there exists a geometric point $\mathcal{L} \in J_{X}^{(1)}(K)$ such that $\theta=\theta_{\mathcal{L}}$ if and only if $\delta_{\theta}=0$.

Proof. This follows immediately from the theory of $[\mathrm{BK}], \S 3$, especially Example 3.11, plus the following observation: If $H$ is (as in Section 3) the $\Gamma_{K}$-module that arises as the abelianization of $\Delta_{X}$, then

$$
\operatorname{Ker}\left\{H^{1}(K, H) \rightarrow H^{1}\left(K, H \otimes B_{D R}^{+}\right)\right\}
$$

(i.e., by Lemma 3.8 .1 of $[\mathrm{BK}]$, " $H_{g}^{1}$ " in the notation of $[\mathrm{BK}]$ ) is equal to

$$
\operatorname{Ker}\left\{H^{1}(K, H) \rightarrow H^{1}(K, H \otimes \widehat{\bar{K}})=H^{1}\left(K, \mathfrak{Z}_{X}[0]\right)\right\}
$$

(where the last equality follows from Proposition 2.4 and Lemma 2.2 (ii) (for $n=1, j=1$ )). Indeed, this equality of kernels follows by using the natural filtration on $B_{D R}^{+}$(whose subquotients are equal to $\widehat{\bar{K}}(i), i \geq 0)$, together with the fact that $H^{1}(K, H \otimes \widehat{\bar{K}}(i))=0$ for $i>0$ (by Proposition 2.4, Lemma 2.2 (ii) (for $n=1, j>0$ )). 
Now, we return to considering the section $\alpha: \Gamma_{K} \rightarrow \Pi_{X_{K}}$. Let $\beta: \Gamma_{K} \rightarrow \Pi_{X_{K}}$ be any section that arises from some geometric point $x \in X_{K}(K)$. We would like to compare $\alpha$ and $\beta$, and, in particular, show that, under the assumption $(*)^{\mathrm{spl}}, \alpha$ and $\beta$ are, in some sense (to be specified precisely below), relatively close. Let $\zeta: \Gamma_{K} \rightarrow \Delta_{X}$ be the continuous function (not necessarily a group homomorphism) such that

$$
\alpha(\gamma)=\zeta(\gamma) \cdot \beta(\gamma) \in \Pi_{X_{K}}
$$

for all $\gamma \in \Gamma_{K}$.

Now let $\phi \in Z_{X}(\widehat{\bar{K}})$. If $\gamma \in \Gamma_{K}$, then let us write $\gamma_{\alpha}(\phi) \in Z_{X}(\widehat{\bar{K}})$ (respectively, $\gamma_{\beta}(\phi) \in Z_{X}(\widehat{\bar{K}})$ ) for the result of letting $\gamma$ act on $\phi$ by means of the action defined by $\alpha$ (respectively, $\beta$ ). Note that it follows from the construction of Section 3 that we have a natural morphism $\Delta_{X} \rightarrow Z_{X}(\widehat{\bar{K}})$. For $\epsilon \in \Delta_{X}$, let us denote by $\epsilon_{Z} \in Z_{X}(\widehat{\bar{K}})$ the image of $\epsilon$ in $Z_{X}(\widehat{\bar{K}})$. Then we have the following:

Lemma 4.2. We have: $\gamma_{\alpha}(\phi)=\zeta(\gamma)_{Z} \cdot \gamma_{\beta}(\phi) \cdot \zeta(\gamma)_{Z}^{-1}$

Proof. Indeed, this follows immediately from the fact that the respective actions of $\Gamma_{K}$ on $Z_{X}(\widehat{\bar{K}})$ are induced by conjugation by $\alpha(\gamma)$ and $\beta(\gamma)$ inside $\Pi_{X_{K}}$.

Let $\alpha^{\prime}: \Gamma_{K} \rightarrow \Pi_{X_{K}}$ be any section of $\Pi_{X_{K}} \rightarrow \Gamma_{K}$, and let $\alpha_{Z}^{\prime}: \Gamma_{K} \rightarrow \Pi_{J_{X}^{(1)}}^{Z}$ be the section obtained by composing $\alpha^{\prime}$ with $\Pi_{X_{K}} \rightarrow \Pi_{J_{X}^{(1)}}^{Z}$. Then we make the following

Definition 4.3. We shall call $\alpha^{\prime} J$-geometric if $\alpha_{Z}^{\prime}$ coincides with $\theta_{\text {geom }}$ (up to composition with an inner automorphism of $\Pi_{J_{X}^{(1)}}^{Z}$ arising from $\mathfrak{Z}_{X}[0] \subseteq \Pi_{J_{X}^{(1)}}^{Z}$ ).

The following result is the main technical observation that made it possible to substantially strengthen the result of [Mzk2].

Proposition 4.4. Let $\alpha: \Gamma_{K} \rightarrow \Pi_{X_{K}}$ be a continuous group homomorphism that defines a section of $\Pi_{X_{K}} \rightarrow \Gamma_{K}$. Then $\alpha$ satisfies $(*)^{\mathrm{spl}}$ if and only if $\alpha$ is J-geometric.

Proof. Let $\phi \in Z_{X}(\widehat{\bar{K}})$ be $\beta$-invariant, i.e., invariant under the action of $\Gamma_{K}$ on $Z_{X}(\widehat{\bar{K}})$ defined by $\beta$. We would like to calculate the action of $\Gamma_{K}$ on $\phi$ that is induced by $\alpha$. Thus, for $\gamma \in \Gamma_{K}$, we have, by Lemma 4.2:

$$
\begin{aligned}
\gamma_{\alpha}(\phi) & =\zeta(\gamma)_{Z} \cdot \gamma_{\beta}(\phi) \cdot \zeta(\gamma)_{Z}^{-1} \\
& =\zeta(\gamma)_{Z} \cdot \phi \cdot \zeta(\gamma)_{Z}^{-1}
\end{aligned}
$$


Thus, $\gamma_{\alpha}(\phi) \cdot \phi^{-1}=\left[\zeta(\gamma)_{Z}, \phi\right]$ (where the brackets denote the "commutator"). Note that

$$
\left[\zeta(\gamma)_{Z}, \phi\right] \in Z_{X}[1](\widehat{\bar{K}})=\mathfrak{Z}_{X}[1]=\wedge^{2} \mathfrak{Z}_{X}[0]=\left(\wedge^{2} \mathcal{H}_{0}\right) \otimes_{K} \widehat{\bar{K}}
$$

Now it remains to reinterpret the calculation just performed in terms of cohomology classes.

Recall the class

$$
\eta_{\alpha} \in \mathcal{H}_{0}^{\vee} \otimes_{K} \wedge^{2} \mathcal{H}_{0}=\operatorname{Hom}_{K}\left(\mathcal{H}_{0}, \wedge^{2} \mathcal{H}_{0}\right)
$$

discussed in Section 3. Let $\phi[0]$ be the image of $\phi \in Z_{X}(\widehat{\bar{K}})$ in $Z_{X}[0](\widehat{\bar{K}})=\mathfrak{Z}_{X}[0]$. Since $\phi$ is $\beta$-invariant, it follows that $\phi[0]$ is a $\Gamma_{K}$-invariant element of $\mathfrak{Z}_{X}[0]$, hence belongs to $\mathcal{H}_{0} \subseteq \mathcal{H}_{0} \otimes_{K} \widehat{\bar{K}}=\mathfrak{Z}_{X}[0]$. Let $\delta_{\alpha} \stackrel{\text { def }}{=} \alpha_{Z}-\theta_{\text {geom }} \in H^{1}\left(K, \mathfrak{Z}_{X}[0]\right)=\mathcal{H}_{0}$. Then it is immediate from the definitions that the calculation of the preceding paragraph, interpreted in terms of cohomology classes, becomes:

$$
\eta_{\alpha}(\phi[0])=\delta_{\alpha} \wedge(\phi[0])
$$

(Note that since $\theta_{\text {geom }}$ may be computed - cf. the discussion preceding Lemma 4.1 - using the geometric section $\beta$, it follows that $\delta_{\alpha}$ is precisely the cohomology class defined by the image of the cocycle $\zeta(-)_{Z}: \Gamma_{K} \rightarrow Z_{X}(\widehat{\bar{K}})$ in $Z_{X}[0](\widehat{\bar{K}})=\mathfrak{Z}_{X}[0]$.) Next observe that this equation holds for all $\beta$-invariant $\phi$, and that every element of $\mathcal{H}_{0} \subseteq \mathcal{H}_{0} \otimes_{K} \widehat{\bar{K}}=\mathfrak{Z}_{X}[0]=$ $Z_{X}[0](\widehat{\bar{K}})$ lifts to a $\beta$-invariant $\phi \in Z_{X}(\widehat{\bar{K}})$. (Indeed, this follows from Proposition 3.5 since $\beta$ arises from a point of $X_{K}(K)$.) Thus, it follows that $\eta_{\alpha}: \mathcal{H}_{0} \rightarrow \mathcal{H}_{0} \wedge \mathcal{H}_{0}$ is simply the map " $\delta_{\alpha} \wedge$." In particular, $\eta_{\alpha}=0$ if and only if $\delta_{\alpha}=0$. This completes the proof of the Proposition.

Remark. So far here we have been dealing with the truncated weight zero quotient $\Delta_{X} \rightarrow$ $Z_{X}$, but it would be interesting also to see what happens in the case of the full nontruncated weight zero quotient $\Delta_{X} \rightarrow Z_{X}^{\infty}$ (as in the Remark at the end of Section 3). For instance, if the action of $\alpha$ on $Z_{X}^{\infty}$ is such that $Z_{X}^{\infty}$ has "enough invariants" (i.e., there exists a proalgebraic group $G$ over $K$ such that $G \otimes_{K} \widehat{\bar{K}}$ is $\Gamma_{K}$-equivariantly isomorphic to $Z_{X}^{\infty}$ ), does it follow that $\alpha$ itself automatically comes from a geometric point $x \in X_{K}(K)$ ? Although such questions are beyond the scope of this paper, it is the opinion of the author that such questions deserve further study. Note that this sort of issue is closely related to the so-called Section Conjecture - cf. the Remark following Theorem 19.1 for more details on this conjecture. 


\section{Section 5: The J-Geometricity of K-Valued Points}

Let $K$ be a $p$-adic field with perfect residue field. Let $X_{K}$ and $Y_{K}$ be proper hyperbolic curves over $K$. Let $U_{K}$ be the $K$-scheme obtained by localizing $X_{K}$ at its generic point. Thus, the underlying topological space of $U_{K}$ consists of one point, and the ring of functions on $U_{K}$ is the function field $K_{X}$ of $X_{K}$. Note in particular that we can consider $\Pi_{U_{K}}, \Pi_{Y_{K}}$. In particular, $\Pi_{U_{K}}$ is a certain quotient of the absolute Galois group of $K_{X}$. Let us assume that we are given a continuous open homomorphism

$$
\theta: \Pi_{U_{K}} \rightarrow \Pi_{Y_{K}}
$$

over $\Gamma_{K}$. In this Section, we would like to begin the proof of the main theorem of this paper by showing that any such $\theta$ necessarily "maps geometric sections to $J$-geometric sections." (Naturally, we will explain below precisely what is meant by the expression in quotes.)

First observe that if $x \in X_{K}(K)$ is any $K$-valued point, then we can form the completion $\left(K_{X}\right)_{x}$ of the field $K_{X}$ with respect to the valuation defined by $x$. Moreover, we have a natural morphism $\Pi_{\left(K_{X}\right)_{x}} \rightarrow \Pi_{U_{K}}$ (well-defined up to composition with conjugation by an element of $\Delta_{U}$ ) whose image is "the" (more rigorously: any of the various conjugate) decomposition group associated to $x$. Moreover, as is well-known (see, e.g., [Ser2]), $\left(K_{X}\right)_{x}$ is (noncanonically) isomorphic to $K((t))$ (where $t$ is an indeterminate), so $\Delta_{\left(K_{X}\right)_{x}}$ may be identified with $\mathbf{Z}_{p}(1)$. In particular, by forming, relative to some isomorphism $\left(K_{X}\right)_{x} \cong K((t))$, the field extension of $\left(K_{X}\right)_{x}$ corresponding to adjoining a compatible system of $p$-power roots of $t$ to $K((t))$, one sees immediately that $\Pi_{\left(K_{X}\right)_{x}} \rightarrow \Gamma_{K}$ admits many sections.

Definition 5.1. We shall refer to as geometric any section $\Gamma_{K} \rightarrow \Pi_{U_{K}}$ of $\Pi_{U_{K}} \rightarrow \Gamma_{K}$ obtained by composing a section of $\Pi_{\left(K_{X}\right)_{x}} \rightarrow \Gamma_{K}$ with (any one of the conjugate natural homomorphisms) $\Pi_{\left(K_{X}\right)_{x}} \rightarrow \Pi_{U_{K}}$.

Note, in particular, that $\Pi_{\left(K_{X}\right)_{x}} \rightarrow \Pi_{X_{K}}$ factors through $\Gamma_{K}$, so in particular, the composite $\alpha_{X}: \Gamma_{K} \rightarrow \Pi_{X_{K}}$ with $\Pi_{U_{K}} \rightarrow \Pi_{X_{K}}$ of any geometric section $\alpha_{U}: \Gamma_{K} \rightarrow \Pi_{U_{K}}$ is induced by some point $x: \operatorname{Spec}(K) \rightarrow X_{K}$.

Let $\alpha_{U}: \Gamma_{K} \rightarrow \Pi_{U_{K}}$ be a geometric section. By composing $\alpha_{U}$ with $\theta$, we obtain a section $\alpha_{Y}: \Gamma_{K} \rightarrow \Pi_{Y_{K}}$. In this Section, we would like to prove that $\alpha_{Y}$ is necessarily $J$-geometric in the sense of Definition 4.3. To do this, let us first observe that we have a diagram of continuous morphisms

$$
\begin{aligned}
& \Delta_{U} \longrightarrow Z_{X}(\widehat{\bar{K}}) \\
& \left.\downarrow \theta\right|_{\Delta_{U}} \\
& \Delta_{Y} \longrightarrow Z_{Y}(\widehat{\bar{K}})
\end{aligned}
$$


which are compatible with the $\Gamma_{K}$-actions defined by $\alpha_{U}$ and $\alpha_{Y}$ on the all the groups involved. (Here, the upper horizontal morphism is obtained by composing $\Delta_{U} \rightarrow \Delta_{X}$ with $\Delta_{X} \rightarrow Z_{X}(\widehat{\bar{K}})$.) Then we have the following

Lemma 5.2. There exists a (natural) surjective $\Gamma_{K}$-homomorphism $Z_{X}(\widehat{\bar{K}}) \rightarrow Z_{Y}(\widehat{\bar{K}})$ that makes the above diagram commute.

Proof. Observe that the kernel of $\Delta_{U} \rightarrow \Delta_{X}$ is generated by inertia groups (i.e., images of the various $\left.\Delta_{\left(K_{X}\right)_{x}} \rightarrow \Delta_{U}\right)$, and the action of $\Gamma_{K}$ on an inertia group is via the cyclotomic character, i.e., (in the language of Hodge-Tate Galois representations) of weight one. It thus follows (from Lemma 2.2 (ii), for $n=0, j=-1$ ) that the morphism $\Delta_{U} \rightarrow Z_{Y}(\widehat{\bar{K}})$ (obtained from the diagram above) factors through $\Delta_{X}$. Hence, we obtain a morphism $\Delta_{X} \rightarrow Z_{Y}(\widehat{\bar{K}})$. But now, it follows immediately (by the universal property of the truncated Malčev completion, plus "weight arguments") from the construction of $Z_{X}(\widehat{\bar{K}})$ from $\Delta_{X}$ that this morphism $\Delta_{X} \rightarrow Z_{Y}(\widehat{\bar{K}})$ factors naturally through $Z_{X}(\widehat{\bar{K}})$. This shows the existence of a morphism as claimed in the statement of the Lemma. The fact that this morphism is surjective follows from the fact that $\theta$ is open (which implies that the induced morphism $\Delta_{U}^{\mathrm{ab}} \rightarrow \Delta_{Y}^{\mathrm{ab}}$ on abelianizations is open, hence surjective after tensoring with $\left.\mathbf{Q}_{p}\right)$.

Lemma 5.3. The action of $\Gamma_{K}$ on $Z_{Y}(\widehat{\bar{K}})$ defined by $\alpha_{Y}$ satisfies the condition $(*)^{\mathrm{spl}}$ discussed in Section 4.

Proof. Indeed, that the action of $\Gamma_{K}$ on $Z_{X}(\widehat{\bar{K}})$ defined by $\alpha_{X}$ satisfies $(*)^{\text {spl }}$ follows from Proposition 3.5. Thus, Lemma 5.3 follows from the surjectivity of the morphism of Lemma 5.2.

Proposition 5.4. Let $\theta: \Pi_{U_{K}} \rightarrow \Pi_{Y_{K}}$ be a continuous open homomorphism over $\Gamma_{K}$, $\alpha_{U}: \Gamma_{K} \rightarrow \Pi_{U_{K}}$ a geometric (Definition 5.1) section, and $\alpha_{Y}: \Gamma_{K} \rightarrow \Pi_{Y_{K}}$ the composite of $\alpha_{U}$ with $\theta$. Then $\alpha_{Y}$ is J-geometric (Definition 4.3).

Proof. This follows by combining Lemma 5.3 with Proposition 4.4.

\section{Section 6: F-Geometricity and FI-Geometricity}

Let $K$ be a finite extension of $\mathbf{Q}_{p}$. Let $S \stackrel{\text { def }}{=} \operatorname{Spec}(R)$, where $R$ is an $\mathcal{O}_{K}$-algebra noncanonically isomorphic to $\mathcal{O}_{K}[[t]]$ (and $t$ is an indeterminate). Let $\eta_{S}$ be the generic 
point of $S$ (regarded as a scheme). Let $\Gamma_{S} \stackrel{\text { def }}{=} \pi_{1}\left(S_{K}\right)$ (where $S_{K} \stackrel{\text { def }}{=} S \otimes_{\mathcal{O}_{K}} K$, and the " $\pi_{1}$ " is with respect to some base-point which we omit to simplify notation); let $\Gamma_{\eta_{S}}$ be the absolute Galois group of $K\left(\eta_{S}\right)$ (the function field of $S$ ). Thus, $\Gamma_{S}$ can be naturally regarded as a quotient of $\Gamma_{\eta_{S}}$. If $H \subseteq \Gamma_{\eta_{S}}$ is an open subgroup corresponding to some finite étale covering $\eta_{S^{\prime}} \rightarrow \eta_{S}$, then we shall write $\Gamma_{\eta_{S^{\prime}}}$ for $H ; S^{\prime}=\operatorname{Spec}\left(R^{\prime}\right)$ for the normalization of $S$ in $\eta_{S^{\prime}}$; and $\Gamma_{S^{\prime}}$ for the quotient of $\Gamma_{\eta_{S^{\prime}}}$ corresponding to étale coverings of $S_{K}^{\prime} \stackrel{\text { def }}{=} S^{\prime} \otimes \mathcal{O}_{K} K$. Thus, $S^{\prime}$ is finite and flat (since $S$ is regular of dimension 2 , and $R^{\prime}$ is normal, hence an $R$-module of depth 2) over $S$.

Remark. For the reader familiar with [Mzk2], we remark that this Section and the next are, in some sense, a generalization of Section 5 of [Mzk2], and play a comparable role in the present paper to that of Section 5 in [Mzk2]. For the reader not familiar with [Mzk2], we remark that, nevertheless, we do not assume any knowledge of [Mzk2] in the following discussion.

We begin by considering a continuous $\mathbf{Z}_{p}\left[\Gamma_{\eta_{S}}\right]$-module $V$, where $V$, as a $\mathbf{Z}_{p}$-module, is a finite and free.

Definition 6.1. We shall refer to $V$ as potentially geometric if there exists some open subgroup $\Gamma_{\eta_{S^{\prime}}} \subseteq \Gamma_{\eta_{S}}$ such that the $\Gamma_{\eta_{S^{\prime}}}$-module obtained by restricting the $\Gamma_{\eta_{S}}$-action on $V$ to $\Gamma_{\eta_{S^{\prime}}}$ arises as the Tate module of some $p$-divisible group $G \rightarrow \operatorname{Spec}\left(\mathcal{O}_{K^{\prime}}\right)$, where $K^{\prime} \subseteq R_{K}^{\prime}$ is a finite extension of $K$.

Note, in particular, that the $\Gamma_{\eta_{S^{\prime}}}$-action on $V$ then factors through the quotient $\Gamma_{\eta_{S^{\prime}}} \rightarrow \Gamma_{S^{\prime}}$. Moreover, if $V$ is potentially geometric, then we may make the following construction: Let us write $\mathcal{G}$ for the (not necessarily connected) formal group (over $\mathcal{O}_{K^{\prime}}$ ) associated to the $p$-divisible group $G \rightarrow \operatorname{Spec}\left(\mathcal{O}_{K^{\prime}}\right)$ of Definition 6.1. Thus, we have a natural isomorphism (of finite flat group schemes over $\mathcal{O}_{K^{\prime}}$ ) between the kernels

$$
\mathcal{G}\left[p^{n}\right] \cong G\left[p^{n}\right]
$$

of multiplication by $p^{n}$ (for all $n \geq 0$ ) on $\mathcal{G}$ and $G$. In particular, if we consider the exact sequence (generalizing the Kummer sequence, which corresponds to the case where $\mathcal{G}$ is the formal group associated to the multiplicative group $\mathbf{G}_{m}$ )

$$
0 \longrightarrow \mathcal{G}\left[p^{n}\right] \longrightarrow \mathcal{G} \stackrel{p^{n}}{\longrightarrow} \mathcal{G} \longrightarrow 0
$$

as an exact sequence of sheaves on the finite flat site of $S^{\prime}$, then we get a natural map

$$
\mathcal{G}\left(S^{\prime}\right) \rightarrow H_{\text {flat }}^{1}\left(S^{\prime}, \mathcal{G}\left[p^{n}\right]\right)
$$


(where the cohomology group is relative to the finite flat topology on $S^{\prime}$ ) for $n \geq 0$. Since étale morphisms are quasi-finite and flat, we also have a natural morphism

$$
H_{\text {flat }}^{1}\left(S^{\prime}, \mathcal{G}\left[p^{n}\right]\right) \rightarrow H_{\text {et }}^{1}\left(S^{\prime}, \mathcal{G}\left[p^{n}\right]_{K^{\prime}}\right)
$$

Thus, if we compose the above two morphisms, take the inverse limit with respect to $n$, and use the fact that $H_{\mathrm{et}}^{1}\left(S^{\prime},-\right) \cong H^{1}\left(\Gamma_{S^{\prime}},-\right)$, we get a natural morphism

$$
\kappa_{\mathcal{G}}: \mathcal{G}\left(S^{\prime}\right) \rightarrow H^{1}\left(\Gamma_{S^{\prime}}, V\right)
$$

which one may regard as a generalization of the Kummer map (from units of a field to a certain Galois cohomology group of the field).

Remark. Note that when the formal group $\mathcal{G}$ arises from an abelian variety over $\mathcal{O}_{K^{\prime}}$, then the cohomology class that one obtains by applying $\kappa_{\mathcal{G}}$ to a point of $\mathcal{G}$ coincides with the cohomology class that one obtains (cf. the discussion of Section 4) by looking at the morphism induced on arithmetic fundamental groups by the corresponding point of the abelian variety. Indeed, this is a matter of general nonsense - cf., e.g., [Naka2], Claim (2.2); [NTs], Lemma (4.14).

Definition 6.2. $\quad$ Suppose that $V$ is potentially geometric, and that $\Gamma_{\eta_{S^{\prime}}} \subseteq \Gamma_{\eta_{S}}$ is as in Definition 6.1. Then we define

$$
H_{f}^{1}\left(S_{K}^{\prime}, V\right) \subseteq H^{1}\left(S_{K}^{\prime}, V\right) \stackrel{\text { def }}{=} H^{1}\left(\Gamma_{S^{\prime}}, V\right)
$$

to be the subset of elements $\zeta \in H^{1}\left(S_{K}^{\prime}, V\right)$ such that some nonzero multiple of $\zeta$ lies in the image of $\mathcal{G}\left(S^{\prime}\right)$ under the morphism $\kappa_{\mathcal{G}}$.

Now let $X_{\eta_{S}} \rightarrow \eta_{S}$ be a proper hyperbolic curve over $\eta_{S}$ such that for some open $\Gamma_{\eta_{S^{\prime}}} \subseteq \Gamma_{\eta_{S}}$, and some proper, hyperbolic curve $Z \rightarrow \operatorname{Spec}\left(K^{\prime}\right)$ (where $K^{\prime} \subseteq R_{K}^{\prime}$ is a finite extension of $K$, and we assume that $Z\left(K^{\prime}\right) \neq \emptyset$ ), we have an isomorphism of $\eta_{S^{\prime}}$-curves

$$
X_{\eta_{S}} \times_{\eta_{S}} \eta_{S^{\prime}} \cong Z \times_{K^{\prime}} \eta_{S^{\prime}}
$$

Then we make the following technical

Definition 6.3. We shall call $X_{\eta_{S}}$ irreducibly splittable if for some open $\Gamma_{\eta_{S^{\prime}}} \subseteq \Gamma_{\eta_{S}}$, and some proper, hyperbolic curve $Z \rightarrow \operatorname{Spec}\left(K^{\prime}\right)$ (where $K^{\prime} \subseteq R_{K}^{\prime}$ is a finite extension of $K$, and we assume that $\left.Z\left(K^{\prime}\right) \neq \emptyset\right)$, we have an isomorphism of $\eta_{S^{\prime}}$-curves

$$
X_{\eta_{S}} \times_{\eta_{S}} \eta_{S^{\prime}} \cong Z \times_{K^{\prime}} \eta_{S^{\prime}}
$$


and, moreover, the (induced) map $S^{\prime} \rightarrow \operatorname{Spec}\left(\mathcal{O}_{K^{\prime}}\right)$ satisfies: (i) $S^{\prime} \rightarrow \operatorname{Spec}\left(\mathcal{O}_{K^{\prime}}\right)$ admits a section; (ii) the morphism $S^{\prime} \rightarrow \operatorname{Spec}\left(\mathcal{O}_{K^{\prime}}\right)$ has geometrically irreducible fibers.

As usual, we have an exact sequence

$$
1 \rightarrow \Delta_{X_{\eta_{S}}}=H_{X} \rightarrow \Pi_{X_{\eta_{S}}} \rightarrow \Gamma_{\eta_{S}} \rightarrow 1
$$

where we write $H_{X}$ for the abelianization of $\Delta_{X_{\eta_{S}}}$. Thus, $H_{X}$ has a natural structure of continuous $\Gamma_{\eta_{S}}$-module. Moreover, as a $\mathbf{Z}_{p}$-module, it is free of rank $2 g_{X}$ (where $g_{X}$ is the genus of $\left.X_{\eta_{S}}\right)$. Let us write

$$
H_{X} \rightarrow H_{X}^{I}
$$

for the quotient of $H_{X}$ by all elements of $H_{X}$ on which some open subgroup of $\Gamma_{\eta_{S}}$ acts via the cyclotomic character. (The " $I$ " comes from the fact that $H_{X}^{I}$ is obtained by forming the quotient of $H_{X}$ by all of its "inertia-like" subgroups.)

Next, let

$$
H_{X}^{F} \subseteq H_{X}
$$

be the largest $\Gamma_{\eta_{S}}$-submodule of $H_{X}$ which has no nonzero torsion-free quotients $H_{X}^{F} \rightarrow Q$ such that some open subgroup of $\Gamma_{\eta_{S}}$ acts trivially on $Q$. (Here, the "F" stands for "finite." This is because $H_{X}^{F}$ corresponds to the portion of $H_{X}$ that (potentially) extends to a $p$-divisible group - i.e., a direct limit of finite flat group schemes - over $\mathcal{O}_{K^{\prime}}$.) Then it is well-known (see, e.g., [FC], Chapter III) that $H_{X}^{F}$ and $H_{X}^{I}$ are Cartier-dual to one another, and, moreover, that $H_{X}^{F}$ is potentially geometric (Definition 6.1). (Indeed, in [FC], Chapter III, one finds a discussion of how one may obtain (in a natural fashion) abelian varieties over $K^{\prime}$ as "quotients" (by some group of periods) of semi-abelian varieties that (potentially) extend over $\mathcal{O}_{K^{\prime}}$ in such a way that the dimensions of their toral parts are the same over the generic and special points of $\mathcal{O}_{K^{\prime}}$. If we apply this theory to the Jacobian $J_{Z}$ of the curve $Z$, then $H_{X}^{F}$ is the Tate module of this semi-abelian variety (that potentially extends over $\mathcal{O}_{K^{\prime}}$ ).) Since $H_{X}^{I}$ is the Cartier dual of $H_{X}^{F}$, it thus follows that $H_{X}^{I}$ is also potentially geometric.

Let

$$
H_{X}^{F I} \subseteq H_{X}^{I}
$$

be the image of $H_{X}^{F}$ in $H_{X}^{I}$. Thus, we have a surjection $H_{X}^{F} \rightarrow H_{X}^{F I}$, and $H_{X}^{F I}$ is also potentially geometric. Finally, let

$$
H_{X}^{M} \stackrel{\text { def }}{=} \operatorname{Ker}\left(H_{X} \rightarrow H_{X}^{I}\right) \subseteq H_{X}^{F}
$$


(Here, the "M" stands for "multiplicative." This is because $H_{X}^{M}$ arises from the portion of $H_{X}^{F}$ that corresponds to twisted copies of (the Tate module of) the multiplicative group $\left.\mathbf{G}_{m}.\right) H_{X}^{M}$ is also potentially geometric.

Next, we would like to consider a section $\alpha: \Gamma_{\eta_{S}} \rightarrow \Pi_{X_{\eta_{S}}}$ of $\Pi_{X_{\eta_{S}}} \rightarrow \Gamma_{\eta_{S}}$. Now recall the isomorphism

$$
X_{\eta_{S}} \times_{\eta_{S}} \eta_{S^{\prime}} \cong Z \times \times_{K^{\prime}} \eta_{S^{\prime}}
$$

By composing $\left.\alpha\right|_{\Gamma_{\eta_{S^{\prime}}}}: \Gamma_{\eta_{S^{\prime}}} \rightarrow \Pi_{X_{\eta_{S}}}$ with the projection $\Pi_{X_{\eta_{S}}} \rightarrow \Pi_{Z}$ induced by this isomorphism, we thus obtain a morphism $\Gamma_{\eta_{S^{\prime}}} \rightarrow \Pi_{Z}$, whose composite with $\Pi_{Z} \rightarrow \Gamma_{K^{\prime}}$ is the natural morphism $\Gamma_{\eta_{S^{\prime}}} \rightarrow \Gamma_{K^{\prime}}$. Now let us make the following assumption on $\alpha$ :

$(*)^{S^{\prime}}$ This morphism $\Gamma_{\eta_{S^{\prime}}} \rightarrow \Pi_{Z}$ factors through $\Gamma_{S^{\prime}}$.

Let us denote the resulting morphism by $\beta: \Gamma_{S^{\prime}} \rightarrow \Pi_{Z}$.

Now let $\gamma: \Gamma_{S^{\prime}} \rightarrow \Pi_{Z}$ be any morphism obtained by composing the natural morphism $\Gamma_{S^{\prime}} \rightarrow \Gamma_{K^{\prime}}$ with some section $\Gamma_{K^{\prime}} \rightarrow \Pi_{Z}$ arising from a geometric point $\in Z\left(K^{\prime}\right)$. Let $\beta_{J}, \gamma_{J}: \Gamma_{S^{\prime}} \rightarrow \Pi_{J_{Z}^{(1)}}$ be the morphisms obtained by composing $\beta$ and $\gamma$, respectively, with $\Pi_{Z} \rightarrow \Pi_{J_{Z}^{(1)}}$. Then the difference $\beta_{J}-\gamma_{J}$ defines an element

$$
\delta_{Z} \in H^{1}\left(S_{K}^{\prime}, H_{Z}\right)
$$

hence an element

$$
\delta_{X} \in H^{1}\left(S_{K}^{\prime}, H_{X}\right)
$$

whose image in $H^{1}\left(S_{K}^{\prime}, H_{X}^{I}\right)$ we denote by $\delta_{X}^{I}$.

Definition 6.4. Suppose that $\alpha: \Gamma_{\eta_{S}} \rightarrow \Pi_{X_{\eta_{S}}}$ is a section that satisfies the assumption $(*)^{S^{\prime}}$ above. Then we shall call $\alpha$ F-geometric (respectively, FI-geometric) if some nonzero multiple of $\delta_{X}$ (respectively, $\delta_{X}^{I}$ ) lies in the image of $H_{f}^{1}\left(S_{K}^{\prime}, H_{X}^{F}\right.$ ) (respectively, $\left.H_{f}^{1}\left(S_{K}^{\prime}, H_{X}^{F I}\right)\right)$ in $H^{1}\left(S_{K}^{\prime}, H_{X}\right)$ (respectively, $H^{1}\left(S_{K}^{\prime}, H_{X}^{I}\right)$ ).

Note that if $\alpha$ is $F$-geometric, it is also automatically $F I$-geometric. Also, let us observe that the definition of " $F$-geometric" or " $F I$-geometric" is independent of the choice of $\gamma$ (cf. Lemma 4.1 and the discussion preceding it). Indeed, to see this, it suffices to verify that some nonzero multiple of the class $\in H^{1}\left(S_{K}^{\prime}, H_{X}\right)$ arising from the difference of two $\gamma^{\prime}$ s lies in $H_{f}^{1}\left(S_{K}^{\prime}, H_{X}^{F}\right)$. But the difference of two $\gamma^{\prime}$ 's (both of which arise from a geometric point $\in Z\left(K^{\prime}\right)$ ) defines a point $\in J_{Z}\left(K^{\prime}\right)$ (where $J_{Z}$ is the Jacobian of $Z$ ). Moreover, since the residue field of $K^{\prime}$ is finite, it follows that some nonzero multiple of this point in $J_{Z}\left(K^{\prime}\right)$ 
extends to an $\mathcal{O}_{K^{\prime}}$-valued point of the Néron model $\mathcal{J}_{Z}$ of $J_{Z}$ over $\mathcal{O}_{K^{\prime}}$ which maps the special point of $\operatorname{Spec}\left(\mathcal{O}_{K^{\prime}}\right)$ to the identity of $\mathcal{J}_{Z}$. On the other hand, since $\mathcal{G}^{F}$ - i.e., the "G" (cf. the discussion following Definition 6.1) for the potentially geometric module $H_{X}^{F}$ - is simply the formal group defined by $\mathcal{J}_{Z}$, it thus follows that such a point $\in \mathcal{J}_{Z}\left(\mathcal{O}_{K^{\prime}}\right)$ defines a point in $\mathcal{G}^{F}\left(\mathcal{O}_{K^{\prime}}\right) \subseteq \mathcal{G}^{F}\left(S^{\prime}\right)$. Thus, by Definition 6.2 , we see that the difference between the two $\gamma^{\text {'s }}$ lies in $H_{f}^{1}\left(S_{K}^{\prime}, H_{X}^{F}\right)$, as desired.

Proposition 6.5. Suppose that $X_{S}$ is irreducibly splittable (cf. Definition 6.3). Then any FI-geometric $\alpha$ is also F-geometric.

Proof. First, let us state that throughout the proof, " $S$ "' will be a fixed $S^{\prime}$ satisfying the conditions of Definition 6.3. Next, let us observe that the cokernel of the natural morphism

$$
H_{f}^{1}\left(S_{K}^{\prime}, H_{X}^{F}\right) \rightarrow H_{f}^{1}\left(S_{K}^{\prime}, H_{X}^{F I}\right)
$$

is torsion. Indeed, this follows from Definition 6.2 and the fact that if $\mathcal{G}^{F}$ and $\mathcal{G}^{F I}$ are the respective formal groups as in Definition 6.2, then the natural morphism $\mathcal{G}^{F} \rightarrow \mathcal{G}^{F I}$ is formally smooth (hence surjective on $S^{\prime}$-valued points).

Thus, if we start with a class $\zeta \in H^{1}\left(S_{K}^{\prime}, H_{X}\right)$ whose image $\zeta^{I} \in H^{1}\left(S_{K}^{\prime}, H_{X}^{I}\right)$ lies in the image of $H_{f}^{1}\left(S_{K}^{\prime}, H_{X}^{F I}\right)$, then (after replacing $\zeta$ by a nonzero multiple of $\zeta$ ), we may assume that there exists a $\zeta^{\prime} \in H^{1}\left(S_{K}^{\prime}, H_{X}\right)$ such that: (a.) $\zeta^{\prime}$ lies in the image of $H_{f}^{1}\left(S_{K}^{\prime}, H_{X}^{F}\right)$; (b.) $\zeta^{\prime}-\zeta$ maps to 0 in $H^{1}\left(S_{K}^{\prime}, H_{X}^{I}\right)$. Moreover, by the definition of $H_{X}^{M} \stackrel{\text { def }}{=} \operatorname{Ker}\left(H_{X} \rightarrow H_{X}^{I}\right)$, it follows that (b.) may be rewritten in the form: " $\zeta$ ' $\zeta$ lies in the image of $H^{1}\left(S_{K}^{\prime}, H_{X}^{M}\right)$ in $H^{1}\left(S_{K}^{\prime}, H_{X}\right)$."

With these observations in hand, it follows that it suffices to prove that

$(*)^{\text {im }}$ The image of $H^{1}\left(S_{K}^{\prime}, H_{X}^{M}\right)$ in $H^{1}\left(S_{K}^{\prime}, H_{X}\right)$ is contained up to torsion (i.e., up to multiplication by a nonzero integer) in the image of $H_{f}^{1}\left(S_{K}^{\prime}, H_{X}^{F}\right)$ in $H^{1}\left(S_{K}^{\prime}, H_{X}\right)$.

Note first that by replacing $K^{\prime}$ by a finite extension of $K^{\prime}$ (and thus also enlarging $S^{\prime}$ note that this does not affect the validity of the conditions (i) and (ii) of Definition 6.3), we may assume that the action of $\Gamma_{S^{\prime}}$ on $H_{X}^{M}(-1)$ (where the " $(-1)$ " is a Tate twist) is trivial. (Thus, in particular, as a $\Gamma_{S^{\prime}}$-module, $H_{X}^{M}$ is isomorphic to a direct sum of a finite number of copies of $\mathbf{Z}_{p}(1)$.) On the other hand, by Lemma 6.6 below (and the Kummer exact sequence), it follows that $H^{1}\left(S_{K}^{\prime}, \mathbf{Z}_{p}(1)\right)=\left\{\left(R_{K}^{\prime}\right)^{\times}\right\}^{\wedge}$ (where the " $\wedge$ " denotes $p$-adic completion) is generated up to torsion by $H_{f}^{1}\left(S_{K}^{\prime}, \mathbf{Z}_{p}(1)\right)=\left\{\left(R^{\prime}\right)^{\times}\right\}^{\wedge}$ and (the image of) $H^{1}\left(K^{\prime}, \mathbf{Z}_{p}(1)\right)=\left\{\left(K^{\prime}\right)^{\times}\right\}^{\wedge}$. Thus, it follows that $H^{1}\left(S_{K}^{\prime}, H_{X}^{M}\right)$ is generated up to torsion by $H_{f}^{1}\left(S_{K}^{\prime}, H_{X}^{M}\right)$ and (the image of) $H^{1}\left(K^{\prime}, H_{Z}^{M}\right)$.

Next, observe that the image of $H^{1}\left(K^{\prime}, H_{Z}^{M}\right)$ in $H^{1}\left(K^{\prime}, H_{Z}\right)$ is contained up to torsion in the image of $H_{f}^{1}\left(K^{\prime}, H_{Z}^{F}\right)$ in $H^{1}\left(K^{\prime}, H_{Z}\right)$. Indeed, this follows from the theory of [BK], 
$\S 3$ (cf. the proof of Lemma 4.1). Namely, since $H_{Z}^{M}$ is "of weight one," the image of $H_{Z}^{M} \subseteq H_{Z}$ in the weight zero component of $H_{Z} \otimes \widehat{\widehat{K}}$ is zero, so any cohomology classes in $H^{1}\left(K^{\prime}, H_{Z}^{M}\right)$ go to zero in $H^{1}\left(K^{\prime},-\right)$ of the weight zero component of $H_{Z} \otimes \mathbf{z}_{p} \widehat{\bar{K}}$. But this means (as we saw in Lemma 4.1), that such cohomology classes arise geometrically, as desired. (Note that, by the above argument involving Néron models (in the discussion immediately preceding Proposition 6.5), it follows that (relative to pulling back cohomology classes over $K^{\prime}$ to cohomology classes over $\left.S_{K}^{\prime}\right)$, the notation " $H_{f}^{1}\left(S_{K}^{\prime},-\right)$ " of Definition 6.2 is consistent with the notation " $H_{f}^{1}\left(K^{\prime},-\right)$ " of $\left.[\mathrm{BK}], \S 3.\right)$

Putting everything together, we thus see that $(*)^{\text {im }}$ has been verified. This completes the proof of the Proposition.

Lemma 6.6. $\quad$ For $S^{\prime}$ satisfying condition (ii) of Definition 6.3, we have that $\left(R_{K}^{\prime}\right)^{\times}$is generated up to torsion by $\left(R^{\prime}\right)^{\times}$and $\left(K^{\prime}\right)^{\times}$.

Proof. Indeed, condition (ii) of Definition 6.3 implies that if $\pi$ is a uniformizing element of $\mathcal{O}_{K^{\prime}} \subseteq R^{\prime}$, then the ideal $\pi \cdot R^{\prime}$ is contained in a unique prime ideal of height one of $R^{\prime}$. Let us denote this prime ideal by $\wp$. Let $x \in R^{\prime}$ be an element which becomes a unit in $R_{K}^{\prime}$. Then $x$ is invertible at every height one prime of $R^{\prime}$ except (possibly) $\wp$. Moreover, by replacing $x$ by some $x^{n}$ (where $n$ is independent of $x$ ), we may assume that $x$ has the same valuation as $\pi^{m}$ (for some nonnegative integer $m$ ) in the discrete valuation ring $R_{\wp}^{\prime}$. It thus follows that $\pi^{-m} \cdot x$ is a unit at every height one prime of $R^{\prime}$. Since $R^{\prime}$ is normal, this implies that $\pi^{-m} \cdot x \in\left(R^{\prime}\right)^{\times}$. This completes the proof of the Lemma.

\section{Section 7: From F-Geometricity to Line Bundles}

In this Section, we use some elementary algebraic geometry (Lemmas 7.1 and 7.2) to translate the rather abstract and technical condition of " $F$-geometricity" into a more tractable existence criterion (Proposition 7.4) for line bundles. Let $S$ be the spectrum of a field of characteristic zero. Let $X \rightarrow S$ be a proper hyperbolic curve of genus $g$ over $S$. Let $N$ be a fixed positive integer. We would like to consider the natural morphism $X \rightarrow J_{X}^{(N)}$ (given by mapping a point $x$ of $X$ to the line bundle $\mathcal{O}_{X}(N \cdot x)$ ). Taking the product of this morphism with $X$ (on the right), we obtain a morphism $\xi: X \times_{S} X \rightarrow J_{X}^{(N)} \times_{S} X$.

Lemma 7.1. There exists a line bundle on $J_{X}^{(N)} \times_{S} X$ whose pull-back via $\xi$ is a nonzero tensor power of the line bundle $\mathcal{D} \stackrel{\text { def }}{=} \mathcal{O}_{X \times_{S} X}(\Delta)$ (where $\Delta \subseteq X \times_{S} X$ ) on $X \times_{S} X$.

Proof. First, let us take the product of $\xi$ with one more copy of $X$ on the right, to obtain a morphism $\xi^{\prime}: X \times_{S} X \times_{S} X \rightarrow J_{X}^{(N)} \times_{S} X \times_{S} X$. For $i, j=1,2,3$ such that 
$i \neq j$, let $\Delta_{i j} \subseteq X \times_{S} X \times_{S} X$ denote the diagonal given by setting equal the $i^{\text {th }}$ and $j^{\text {th }}$ components of the triple product. Let $\mathcal{D}_{i j}$ denote the line bundle $\mathcal{O}_{X \times{ }_{S} X \times{ }_{S} X}\left(\Delta_{i j}\right)$. In the triple product of $X$, we consider the second $X$ to be the "true curve $X$ "; the first $X$ to be a "parameter space for the family of line bundles $\mathcal{D}_{12}$ " (regarded as line bundles on the "true curve" given by the second factor); and the third $X$ to be a "base extension $X \rightarrow S$." Over this extended base, the "true curve" acquires a "section" $\Delta_{23}$. Thus, it follows from the general theory of the Picard functor of a family of curves for which a section exists that there exists a line bundle $\mathcal{L}^{\prime}$ on $J_{X}^{(N)} \times_{S} X \times_{S} X$ whose pull-back via $\xi^{\prime}$ is equal to $\mathcal{E}^{\prime} \stackrel{\text { def }}{=}\left(\mathcal{D}_{12} \otimes \mathcal{D}_{13}^{-1}\right)^{\otimes N}$.

Now let us consider the "determinant of the higher direct image sheaves" (cf. [MB], $\S 1$, for an exposition of this notion) of $\mathcal{L}^{\prime}$ and $\mathcal{E}^{\prime}$ for the morphisms $X \times{ }_{S} X \times{ }_{S} X \rightarrow X \times{ }_{S} X$ and $J_{X}^{(N)} \times_{S} X \times_{S} X \rightarrow J_{X}^{(N)} \times_{S} X$ given by forgetting the third factor. We denote the respective "determinants of the higher direct image sheaves" of $\mathcal{L}^{\prime}$ and $\mathcal{E}^{\prime}$ by $\mathcal{L}$ and $\mathcal{E}$. Thus, (by the functoriality of forming the "determinant of the higher direct image sheaves") $\mathcal{L}$ is a line bundle on $J_{X}^{(N)} \times{ }_{S} X$ such that $\xi^{*}(\mathcal{L})=\mathcal{E}$. Moreover, I claim that we can write

$$
\mathcal{E}=\mathcal{D}^{\otimes N} \otimes \mathcal{F}
$$

where $\mathcal{F}$ is a line bundle on $X \times_{S} X$ obtained by pulling back some $\omega_{X / S}^{\otimes m}$ (for $m \in \mathbf{Z}$ ) from the first factor of $X \times_{S} X$, and tensoring with the pull-back to $X \times_{S} X$ of some line bundle $\mathcal{M}$ on $S$. Indeed, the " $\mathcal{D}^{\otimes N}$-term" arises from the fact that $\mathcal{D}_{12}$ is the pullback of $\mathcal{D}$ via the projection $X \times_{S} X \times_{S} X \rightarrow X \times_{S} X$ under consideration. Then the difference between the "determinants of the higher direct image sheaves" of the line bundles $\mathcal{D}_{13}^{-N}=\mathcal{O}_{X \times_{S} X \times_{S} X}\left(-N \cdot \Delta_{13}\right)$ and $\mathcal{O}_{X \times_{S} \times_{S} X}$ may be computed using the natural inclusion

$$
\mathcal{D}_{13}^{-N}=\mathcal{O}_{X \times{ }_{S} X \times{ }_{S} X}\left(-N \cdot \Delta_{13}\right) \subseteq \mathcal{O}_{X \times{ }_{S} \times{ }_{S} X}
$$

By using the fact that the "determinant of the higher direct image sheaves" is multiplicative on exact sequences, we thus obtain that this difference is equal to some power of the pullback to $X \times_{S} X$ (via the projection to the first factor) of $\left.\mathcal{O}_{X \times_{S} X}(-\Delta)\right|_{\Delta=X}=\omega_{X / S}$. On the other hand, the "determinant of the higher direct image sheaves" of the trivial line bundle on $X \times_{S} X \times_{S} X$ is clearly the pull-back (to $X \times_{S} X$ ) of a line bundle $\mathcal{M}$ on $S$. This gives us a line bundle $\mathcal{F}$ of the form discussed above, hence completes the proof of the claim.

By replacing $\mathcal{L}$ with $\mathcal{L} \otimes_{\mathcal{O}_{S}} \mathcal{M}^{-1}$, we may assume that $\mathcal{M}$ is trivial. Moreover, by Lemma 7.2 below, there exists a line bundle $\mathcal{P}$ on $J_{X}^{(N)}$ whose pull-back to $X$ (via the natural morphism $X \rightarrow J_{X}^{(N)}$ ) is some nonzero tensor power of $\omega_{X / S}$. Thus, replacing $\mathcal{L}$ by a tensor product of appropriate powers of $\mathcal{L}$ and $\left(\left.\mathcal{P}\right|_{J_{X}^{(N)} \times_{S} X}\right)$ completes the proof of the Lemma. 
Lemma 7.2. There exists a line bundle $\mathcal{P}$ on $J_{X}^{(N)}$ whose pull-back to $X$ is a nonzero tensor power of $\omega_{X / S}$.

Proof. Let us first consider the case $N=g-1$. In this case, we take $\mathcal{P}$ to be the line bundle on $J_{X}^{(g-1)}$ defined by the natural theta divisor on $J_{X}^{(g-1)}$ (i.e., the image of the natural morphism from the $(g-1)$-fold product of $X$ to $\left.J_{X}^{(g-1)}\right)$. Then the fact that the pull-back of $\mathcal{P}$ to $X$ is equal to $\omega_{X / S}^{\otimes \frac{1}{2} g(g-1)}$ is an immediate consequence of [MB], Corollary 2.5: in the notation of loc. cit., we are interested here in the case where one takes $n=0$; $S=X$; and $a: S \rightarrow X$ to be the identity; then specializing the formula of loc. cit. to the zero section of the Jacobian (note that the restriction of " $\mathcal{U}_{n}^{(a)}$ " in loc. cit. to the zero section of the Jacobian is trivial) proves the assertion concerning the pull-back of $\mathcal{P}$ to $X$.

Next, let us consider the case where $N$ is divisible by $g-1$. Then the morphism $X \rightarrow J_{X}^{(N)}$ factors through $J_{X}^{(g-1)} \rightarrow J_{X}^{(N)}$. Moreover, it follows from the basic theory of line bundles on abelian varieties (see, e.g., [AV], §23, the Corollary to Theorem 2 on p. 231) that a nonzero tensor power of the " $\mathcal{P}$ " considered in the previous paragraph descends from $J_{X}^{(g-1)}$ to $J_{X}^{(N)}$. This completes the proof in the case where $N$ is divisible by $g-1$.

Finally, we consider the case of arbitrary positive $N$. In this case, we have a natural map $J_{X}^{(N)} \rightarrow J_{X}^{(N(g-1))}$ (multiplication by $g-1$ ). But, by the preceding paragraph, we already have a suitable " $\mathcal{P}$ " on $J_{X}^{(N(g-1))}$. Thus, by pulling this line bundle back to $J_{X}^{(N)}$, we obtain a suitable "P्P" on $J_{X}^{(N)}$. This completes the proof of the Lemma.

Next, we would like to consider a section $\alpha: \Gamma_{S} \stackrel{\text { def }}{=} \pi_{1}(S) \rightarrow \Pi_{X}$ of $\Pi_{X} \rightarrow \Gamma_{S}$. By composing $\alpha$ with $\pi_{1}(-)$ applied to $X \rightarrow J_{X}^{(N)}$, we obtain a section $\alpha_{J}^{N}: \Gamma_{S} \rightarrow \Pi_{J_{X}^{(N)}}$.

Lemma 7.3. Suppose that $\alpha_{J}^{N}$ arises from a geometric section $\in J_{X}^{(N)}(S)$. Then there exists a line bundle on $X$ of degree prime to $p$.

Proof. By taking the fibered product (over $\Gamma_{S}$ ) of $\alpha$ with the identity on $\Pi_{X}$, we obtain a morphism $\alpha_{X}: \Pi_{X} \rightarrow \Pi_{X \times_{S} X}$. Since $S$ is the spectrum of the field, the group cohomology of $\Pi_{X \times{ }_{S} X}$ computes the $p$-adic étale cohomology of $X \times_{S} X$ (cf. Lemma 0.4). Thus, we can form the arithmetic first Chern class of the line bundle $\mathcal{D}$ of Lemma 7.1 (cf. Definition 0.3): $c_{1}(\mathcal{D}) \in H^{2}\left(\Pi_{X \times_{S} X}, \mathbf{Z}_{p}(1)\right)$. Let $\zeta \stackrel{\text { def }}{=} \alpha_{X}^{*}\left(c_{1}(\mathcal{D})\right) \in H^{2}\left(\Pi_{X}, \mathbf{Z}_{p}(1)\right)$. On the other hand, by composing $\alpha_{X}$ with $\pi_{1}(-)$ of the morphism $\xi$, we obtain a morphism $\alpha_{\xi}^{N}: \Pi_{X} \rightarrow \Pi_{J_{X}^{(N)} \times_{S} X}$. Moreover, by assumption, $\alpha_{\xi}^{N}$ arises from a geometric morphism $X \rightarrow J_{X}^{(N)} \times_{S} X$. Thus, if $\mathcal{L}$ is a line bundle on $J_{X}^{(N)} \times_{S} X$, we obtain that $\left(\alpha_{\xi}^{N}\right)^{*}\left(c_{1}(\mathcal{L})\right) \in$ $H^{2}\left(\Pi_{X}, \mathbf{Z}_{p}(1)\right)$ can be written as $c_{1}(\mathcal{M})$, for some line bundle $\mathcal{M}$ on $X$. Now recall that by Lemma 7.1, there exists a line bundle $\mathcal{L}$ on $J_{X}^{(N)} \times{ }_{S} X$ such that $\xi^{*} \mathcal{L}$ is a nonzero tensor power of $\mathcal{D}$. Thus, putting everything together, it follows that some nonzero multiple of 
the abstract cohomology class $\zeta \in H^{2}\left(\Pi_{X}, \mathbf{Z}_{p}(1)\right)$ can be written in the form $c_{1}(\mathcal{M})$ for some line bundle $\mathcal{M}$ on $X$, i.e., $m \cdot \zeta=c_{1}(\mathcal{M})$ (for some nonzero $m \in \mathbf{Z}$ ). Next, let us recall that since $\zeta$ was constructed from $\mathcal{D}$, the image of $\zeta$ under the "degree map" $H^{2}\left(\Pi_{X}, \mathbf{Z}_{p}(1)\right) \rightarrow H^{2}\left(\Delta_{X}, \mathbf{Z}_{p}(1)\right) \cong \mathbf{Z}_{p}$ is equal to 1 , so $\operatorname{deg}(\mathcal{M})=m$. Thus, we can argue as follows (cf. [Mzk2], Lemma 6.1): Write $m=a \cdot p^{b}$, where $a$ is an integer prime to $p$, and $b$ is a nonnegative integer. Thus, $c_{1}(\mathcal{M})$ vanishes in $H^{2}\left(\Pi_{X},\left(\mathbf{Z} / p^{b} \mathbf{Z}\right)(1)\right)$. But, by the Kummer exact sequence, this implies that there exists a line bundle $\mathcal{P}$ on $X$ such that $\mathcal{P}^{\otimes p^{b}} \cong \mathcal{M}$. In particular, $\operatorname{deg}(\mathcal{P})=p^{-b} \cdot \operatorname{deg}(\mathcal{M})=a$, so the existence of $\mathcal{P}$ completes the proof of the Lemma.

Now let us consider the following situation: Let $K$ be a finite extension of $\mathbf{Q}_{p}$. Let $X_{K} \rightarrow \operatorname{Spec}(K)$ and $Y_{K} \rightarrow \operatorname{Spec}(K)$ be proper hyperbolic curves over $K$. Let $U_{K}$ be the generic point of $X_{K}$. Moreover, let us assume that we have been given a continuous open homomorphism over $\Gamma_{K}$

$$
\theta: \Pi_{U_{K}} \rightarrow \Pi_{Y_{K}}
$$

Now let $S, \eta_{S}$ be as at the beginning of Section 6 . Write $X_{\eta_{S}}$ for $X_{K} \times_{K} \eta_{S}$ (and similarly, for $\left.U_{\eta_{S}}, Y_{\eta_{S}}\right)$. Then by base-change (note that $\Pi_{U_{\eta_{S}}}=\Pi_{U_{K}} \times_{\Gamma_{K}} \Gamma_{\eta_{S}} ; \Pi_{Y_{\eta_{S}}}=\Pi_{Y_{K}} \times_{\Gamma_{K}}$ $\left.\Gamma_{\eta_{S}}\right), \theta$ induces a continuous open homomorphism

$$
\theta_{\eta_{S}}: \Pi_{U_{\eta_{S}}} \rightarrow \Pi_{Y_{\eta_{S}}}
$$

Let $Y_{\eta_{S}}^{\prime} \rightarrow Y_{\eta_{S}}$ be a finite étale covering induced by some open subgroup of $\Pi_{Y_{\eta_{S}}}$ that surjects onto $\Gamma_{\eta_{S}}$. Note that $\theta_{\eta_{S}}$ allows us to define the pull-back of $Y_{\eta_{S}}^{\prime} \rightarrow Y_{\eta_{S}}$ to $U_{\eta_{S}}$. Let $U_{\eta_{S}}^{\prime} \rightarrow U_{\eta_{S}}$ be a connected component of this pull-back which is geometrically connected over $\eta_{S}$. Note that $U_{\eta_{S}}^{\prime} \rightarrow U_{\eta_{S}}$ extends to a finite (possibly ramified) covering $X_{\eta_{S}}^{\prime} \rightarrow X_{\eta_{S}}$. Thus, $X_{\eta_{S}}^{\prime}$ is a proper hyperbolic curve over $\eta_{S}$. Let us suppose that $Y_{\eta_{S}}^{\prime}$ is irreducibly splittable (Definition 6.3). Then it follows immediately that $X_{\eta_{S}}^{\prime}$ is also irreducibly splittable. (Indeed, the definition of "irreducibly splittable" only involves the base (i.e., $S, S^{\prime}$, etc.), plus finite étale coverings of the curve, i.e., it may be phrased entirely in terms of (the base plus) fundamental groups. Thus, the fact that $X_{\eta_{S}}^{\prime}$ and $Y_{\eta_{S}}^{\prime}$ are related by $\theta_{\eta_{S}}$ is enough to guarantee that " $Y_{\eta_{S}}^{\prime}$ irreducibly splittable $\Longrightarrow X_{\eta_{S}}^{\prime}$ irreducibly splittable.")

Next, we would like to consider a section

$$
\alpha_{U}: \Gamma_{\eta_{S}} \rightarrow \Pi_{U_{\eta_{S}}^{\prime}}
$$

of $\Pi_{U_{\eta_{S}}^{\prime}} \rightarrow \Gamma_{\eta_{S}}$. Composing $\alpha_{U}$ with $\pi_{1}(-)$ of the natural morphism $U_{\eta_{S}}^{\prime} \rightarrow X_{\eta_{S}}^{\prime}$ gives rise to a section $\alpha_{X}: \Gamma_{\eta_{S}} \rightarrow \Pi_{X_{\eta_{S}}^{\prime}}$. Composing $\alpha_{U}$ with the morphism $\theta_{\eta_{S}}^{\prime}: \Pi_{U_{\eta_{S}}^{\prime}} \rightarrow \Pi_{Y_{\eta_{S}}^{\prime}}$ induced by $\theta_{\eta_{S}}$ gives a section $\alpha_{Y}: \Gamma_{\eta_{S}} \rightarrow \Pi_{Y_{\eta_{S}}^{\prime}}$. 
The following key result is the culmination of our efforts in Sections 1 through 7:

Proposition 7.4. $\quad$ Under the circumstances just described above, let us assume that $\alpha_{X}$ is FI-geometric (Definition 6.4). Then it follows that $Y_{\eta_{S}}^{\prime}$ admits a line bundle of degree prime to $p$.

Proof. First observe that the morphism $\Delta_{U_{\eta_{S}}^{\prime}} \rightarrow \Delta_{Y_{\eta_{S}}^{\prime}} \rightarrow H_{Y_{\eta_{S}}^{\prime}}^{I}$ (where $H_{Y_{\eta_{S}}^{\prime}}^{I}$ is as in the discussion preceding Definition 6.4) factors through $H_{X_{\eta_{S}}^{\prime}}^{I}$. (Indeed, this follows by observing, as in the proof of Lemma 5.2, that the inertia groups of $\Delta_{U_{\eta_{S}}^{\prime}}$ map to zero in $H_{Y_{\eta_{S}}^{\prime}}^{I}$.) Thus, we obtain a $\Gamma_{\eta_{S}}$-equivariant morphism $H_{X_{\eta_{S}}^{\prime}}^{I} \rightarrow H_{Y_{\eta_{S}}^{\prime}}^{I}$. In particular, it follows by using Tate's theorem (i.e., Theorem 4 of [Tate] - that morphisms between Tate modules of $p$-divisible groups induce morphisms between the $p$-divisible groups, hence morphisms between the respective formal groups) that $H_{f}^{1}\left(S_{K}^{\prime}, H_{X_{\eta_{S}}^{\prime}}^{F I}\right)$ maps to $H_{f}^{1}\left(S_{K}^{\prime}, H_{Y_{\eta_{S}}^{\prime}}^{F I}\right)$, for any finite étale covering $\eta_{S^{\prime}} \rightarrow \eta_{S}$ of $\eta_{S}$ as in Definition 6.2.

Next, I claim that $\alpha_{Y}$ is FI-geometric. Indeed, this follows from Proposition 5.4, plus the observation of the preceding paragraph. In words: This claim amounts to the claim (cf. Definition 6.4) that the "J-portion" of $\alpha_{Y}$ (i.e., the result of composing $\alpha_{Y}$ with $\Pi_{Y_{\eta_{S}}^{\prime}} \rightarrow \Pi_{J_{Y^{\prime}}^{(1)}}$, where $J_{Y^{\prime}}^{(1)}$ is the Picard scheme of line bundles of degree 1 on $Y_{\eta_{S}}^{\prime}$ ) differs from the "J-portion" of a constant (i.e., arising from a point defined over a finite extension of $K$ of the curve " $Z$ " of the discussion of Section 6 ) geometric section of $\Pi_{Y_{\eta_{S}}^{\prime}}$ by a class in $H_{f}^{1}\left(S_{K}^{\prime}, H_{Y_{\eta_{S}}^{\prime}}^{F I}\right)$. On the other hand, since we are operating under the assumption that $\alpha_{X}$ is FI-geometric, we know that the $J$-portion of $\alpha_{X}$ differs from the $J$-portion of a constant geometric section of $\Pi_{X_{\eta_{S}}^{\prime}}$ by a class in $H_{f}^{1}\left(S_{K}^{\prime}, H_{X_{\eta_{S}}^{\prime}}^{F^{\prime}}\right)$. Moreover, by Proposition 5.4, constant geometric sections of $\Pi_{U_{\eta_{S}}^{\prime}}$ map to constant $J$-geometric (which is as good as "geometric" for us, since we are only interested in " $J$-portions" here) sections of $\Pi_{Y_{\eta_{S}}^{\prime}}$. Thus, since (by the observation of the preceding paragraph) $H_{f}^{1}\left(S_{K}^{\prime}, H_{X_{\eta_{S}}^{\prime}}^{F I}\right)$ maps to $H_{f}^{1}\left(S_{K}^{\prime}, H_{Y_{\eta_{S}}^{\prime}}^{F I}\right)$, we conclude that $\alpha_{Y}$ is $F I$-geometric, as desired. This completes the proof of the claim. Now since $Y_{\eta_{S}}^{\prime}$ is also assumed to be irreducibly splittable, it follows from Proposition 6.5 that $\alpha_{Y}$ is, in fact, F-geometric.

Now let us consider the morphism $\alpha_{J}^{N}: \Gamma_{\eta_{S}} \rightarrow \Pi_{J_{Y^{\prime}}^{(N)}}$ (where $J_{Y^{\prime}}^{(N)}$ is the Picard scheme of line bundles of degree $N$ on $Y_{\eta_{S}}^{\prime}$ ), for some $N>0$, induced by composing $\alpha_{Y}$ with $\Pi_{Y_{\eta_{S}}^{\prime}} \rightarrow \Pi_{J_{Y^{\prime}}^{(N)}}$. Note that the $F$-geometricity of $\alpha_{Y}$ is, by definition, a property concerning $\alpha_{J}^{1}$. By the definition of $F$-geometricity (Definition 6.4), it follows immediately that if $N$ is large enough, then $\alpha_{J}^{N}$ arises from a geometric section $J_{Y^{\prime}}^{(N)}\left(S_{K}\right)$. Indeed, the large $N$ is to take care of the phrase "nonzero multiple of" in Definitions 6.2 and 6.4, plus the fact that a priori the geometric point whose existence is guaranteed by Definition 6.2 is only defined over $\eta_{S^{\prime}}$, so we may need to apply the norm map (for $\eta_{S^{\prime}} \rightarrow \eta_{S}$ ) to get a 
geometric point over $\eta_{S}$, which may cause $N$ to increase again. Now, applying Lemma 7.3 completes the proof of the Proposition.

Remark. Note that Sections 6 and 7 are made much more technically difficult by the fact that we start with a morphism $\Pi_{U_{K}} \rightarrow \Pi_{Y_{K}}$ rather than a morphism $\Pi_{X_{K}} \rightarrow \Pi_{Y_{K}}$. Indeed, the whole business of distinguishing "F-geometric" from "FI-geometric" arises because one does not have a proper theory of " $p$-divisible groups of infinite rank," which is the sort of object that one must deal with if one tries to work directly with $H_{U}$ (the abelianization of $\Delta_{U}$ ) without passing to $H_{U}^{I}$. Similar technical problems (arising from the fact that $H_{U}$ is of infinite rank) also are the reason behind the lengthiness of Sections 11 and 12 .

\section{Section 8: From Line Bundles to Tame Points}

In this Section, which is something of an appendix to that portion of the paper constituted by Sections 1 through 7, we again apply some elementary algebraic geometry, this time to pass from line bundles to rational points of the curve defined over tamely ramified extensions of the given field. Thus, let $M$ be a $p$-adic field whose residue field $k_{M}$ is isomorphic to $k((t))$, where $k$ is a finite field, and $t$ is an indeterminate. Let $X_{M}$ be a proper hyperbolic curve over $M$. Then we have the following result:

Proposition 8.1. $\quad$ Suppose that $X_{M}$ admits a line bundle $\mathcal{L}$ of degree prime to $p$. Then there exists a tamely ramified extension $M^{\prime}$ of $M$ such that $X_{M}\left(M^{\prime}\right)$ is nonempty.

Proof. By replacing $\mathcal{L}$ by an appropriate (prime to $p$ ) tensor power of the original $\mathcal{L}$, we may assume that $\mathcal{L}$ is very ample. Thus, by Bertini's Theorem, it follows that there exists a divisor $D \subseteq X_{M}$ such that $\mathcal{L} \cong \mathcal{O}_{X_{M}}(D)$, and $D$ is étale over $M$. Let us write

$$
D=\bigcup_{i=1}^{r} D_{i}
$$

where each $D_{i}=\operatorname{Spec}\left(M_{i}\right)$. Thus, $M_{i}$ is a finite field extension of $M$. Since $\operatorname{deg}(D)$ is prime to $p$, it follows that at least one of the $M_{i}$ (say, $M_{1}$ ) is such that $\left[M_{1}: M\right]$ is prime to $p$. Let $M_{c}$ be the Galois closure of $M_{1}$ over $M$. Then it follows from the elementary theory of $p$-adic fields (in particular, the fact that the wild inertia subgroup of $\Gamma_{M}$ is a normal pro- $p$ subgroup of $\Gamma_{M}$, hence contained in any Sylow-pro- $p$ subgroup of $\Gamma_{M}-$ see, e.g., [Ser2], Chapter IV, §2) that $M_{c}$ is tamely ramified over $M$. Thus, in particular, $M_{1}$ is tamely ramified over $M$, and $X_{M}\left(M_{1}\right)$ is nonempty. This completes the proof of the Proposition. 
Next, before continuing, we would like to discuss an auxiliary Lemma that will be useful when we apply Proposition 8.1. Let us suppose that there exists a $p$-adic field $L \subseteq M$ satisfying the following properties:

(1) We have an inclusion $\mathcal{O}_{L} \subseteq \mathcal{O}_{M}$, relative to which $\mathfrak{m}_{L} \cdot \mathcal{O}_{M}=\mathfrak{m}_{M}$.

(2) If $k_{L}$ is the residue field of $L$, then $k \subseteq k_{L}$, and $k_{L}$ is a function field in one variable over $k$.

(3) The inclusion $k_{L} \subseteq k_{M}$ is obtained by completing the function field $k_{L}$ at one of its $k$-valued points.

Let us suppose, moreover, that there exists a curve $X_{L} \rightarrow \operatorname{Spec}(L)$ such that $X_{M}=$ $X_{L} \otimes_{L} M$. Then we have the following result:

Lemma 8.2. Suppose that there exists a tamely ramified extension $M^{\prime}$ of $M$ of ramification index e such that $X_{M}\left(M^{\prime}\right)$ is nonempty. Then there exists a tamely ramified extension $L^{\prime}$ of $L$ of ramification index e such that $X_{L}\left(L^{\prime}\right)$ is nonempty.

Proof. Since tamely ramified extensions of $M$ descend to tamely ramified extensions of $L$, it is easy to see that without loss of generality, we may assume that $e=1$, and $M^{\prime}=M$. Thus, we must show that $X_{L}$ admits a rational point over some unramified extension of $L$. Note that since $\mathcal{O}_{L}$ is a discrete valuation ring, it is well-known that $X_{L}$ admits a regular model $\mathcal{X}_{L} \rightarrow \operatorname{Spec}\left(\mathcal{O}_{L}\right)$. Let $\mathcal{X}_{M} \stackrel{\text { def }}{=} \mathcal{X}_{L} \otimes_{\mathcal{O}_{L}} \mathcal{O}_{M}$. Let us consider the morphism $\phi: \operatorname{Spec}\left(\mathcal{O}_{M}\right) \rightarrow \operatorname{Spec}\left(\mathcal{O}_{L}\right)$. Since finitely generated algebras over a finite field are excellent, it follows that $k_{M}$ is separable over $k_{L}$ (i.e., the morphism $\operatorname{Spec}\left(k_{M}\right) \rightarrow$ $\operatorname{Spec}\left(k_{L}\right)$ is geometrically regular). In particular, it follows that $\phi$ is geometrically regular. Since the natural morphism $\mathcal{X}_{M} \rightarrow \mathcal{X}_{L}$ is obtained from $\phi$ by base-change, it thus follows that it, too, is geometrically regular. Thus, the regularity of $\mathcal{X}_{L}$ implies that of $\mathcal{X}_{M}$. Now the fact that $X_{M}(M)$ is nonempty (by assumption) implies that there exist points in the special fiber of $\mathcal{X}_{M}$ at which $\mathcal{X}_{M}$ is smooth over $\mathcal{O}_{M}$. But this implies (by descent) that there exist points in the special fiber of $\mathcal{X}_{L}$ at which $\mathcal{X}_{L}$ is smooth over $\mathcal{O}_{L}$, which, in turn, implies that for some unramified extension $L^{\prime}$ of $L, X_{L}\left(L^{\prime}\right)$ is nonempty, as desired.

\section{Section 9: Convergence via p-adic Hodge Theory}

Whereas Sections 1 through 8 formed a unit devoted essentially to showing Proposition 7.4, Sections 9 and 10 form a new unit, devoted to showing, by means of $p$-adic Hodge theory, that certain types of sequences of points converge $p$-adically to a uniquely determined point defined over a relatively small field. Thus, in particular, we shall start with fresh hypotheses, as follows: 
Let $K$ be a $p$-adic local field, with residue field $k$. Let $L$ be a $p$-adic field containing $K$ whose residue field $k_{L}$ is a function field in one variable over $k$. Let us also assume that $k$ is algebraically closed in $k_{L}$, and that $\mathfrak{m}_{K} \cdot \mathcal{O}_{L}=\mathfrak{m}_{L}$. Let us denote by

$$
\Gamma_{L / K} \stackrel{\text { def }}{=} \operatorname{Ker}\left(\Gamma_{L} \rightarrow \Gamma_{K}\right)=\Gamma_{L \cdot \bar{K}}
$$

the "geometric" fundamental group of $L$. Let $\mathcal{H}_{L}^{\Omega}$ be the quotient of $H^{1}\left(\Gamma_{L / K}, \widehat{\mathcal{O}}_{\bar{L}}(1)\right)$ by its torsion submodule. Let $X_{K} \rightarrow \operatorname{Spec}(K)$ be a proper hyperbolic curve. Let $X_{L} \stackrel{\text { def }}{=} X_{K} \otimes_{K} L$. Thus, as usual, we have a group extension (obtained by pulling back $1 \rightarrow \Delta_{X} \rightarrow \Pi_{X_{K}} \rightarrow$ $\Gamma_{K} \rightarrow 1$ via $\left.\Gamma_{L} \rightarrow \Gamma_{K}\right)$

$$
1 \rightarrow \Delta_{X} \rightarrow \Pi_{X_{L}} \rightarrow \Gamma_{L} \rightarrow 1
$$

Let us assume that we are given a continuous, group homomorphism

$$
\alpha: \Gamma_{L} \rightarrow \Pi_{X_{K}}
$$

whose composite with the projection to $\Gamma_{K}$ is the natural morphism $\Gamma_{L} \rightarrow \Gamma_{K}$. As usual, $\alpha$ defines a section $\alpha_{s}: \Gamma_{L} \rightarrow \Pi_{X_{L}}$ of $\Pi_{X_{L}} \rightarrow \Gamma_{L}$. Let us also assume that $\alpha$ is nondegenerate in the sense that it satisfies the following group-theoretic condition:

$(*)^{\text {non }}$ The natural morphism $H^{1}\left(\Delta_{X}, \mathbf{Z}_{p}(1)\right) \rightarrow \mathcal{H}_{L}^{\Omega}$ induced by $\alpha$ is nonzero.

Let $L^{\mathrm{tm}}$ be a maximal tamely ramified extension of $L$. Let $M \stackrel{\text { def }}{=}\left(L^{\mathrm{tm}}\right)^{\wedge}$ be its $p$-adic completion. Since $L^{\mathrm{tm}} \cdot \bar{K}$ is unramified over $L \cdot \bar{K}$, and Galois cohomology "ignores unramified extensions," we have that

$$
H^{1}\left(\Gamma_{L / K}, \widehat{\mathcal{O}}_{\bar{L}}(1)\right)=H^{1}\left(\Gamma_{L \cdot \bar{K}}, \widehat{\mathcal{O}}_{\bar{L}}(1)\right)=H^{1}\left(\Gamma_{L^{\mathrm{tm}} \cdot \bar{K}}, \widehat{\mathcal{O}}_{\bar{L}}(1)\right)
$$

In particular, since $\mathcal{H}_{L}^{\Omega}$ is a quotient of this cohomology module, it follows from assumption $(*)^{\text {non }}$ that $\left.\alpha\right|_{\Gamma_{L^{\mathrm{tm}} \cdot \bar{K}}}: \Gamma_{L^{\mathrm{tm}} \cdot \bar{K}} \rightarrow \Delta_{X}$ is nontrivial.

For $n \geq 0$, let $I_{L}^{n} \stackrel{\text { def }}{=} \operatorname{Im}\left(\alpha_{s}\right) \cdot\left(\Delta_{X}\right)<n>\subseteq \Pi_{X_{L}}$ (cf. the discussion preceding Definition 0.2 for an explanation of the notation " $<n>$ "). Let $\psi_{n}: X_{L}^{n} \rightarrow X_{L}$ be the corresponding finite étale covering. Let $X_{L}^{\infty} \rightarrow X_{L}$ be the inverse limit of the $X_{L}^{n}$. In this Section, we would like to prove the following assertion:

$(*)^{\text {con }}$ Suppose that we have a sequence of points $\left\{\widetilde{x}_{n}\right\}$, where $\widetilde{x}_{n} \in$ $X_{L}^{n}\left(L^{\mathrm{tm}}\right)$. Let $x_{n} \stackrel{\text { def }}{=} \psi_{n}\left(\widetilde{x}_{n}\right) \in X_{L}\left(L^{\mathrm{tm}}\right)$. Then there exists a subsequence of $\left\{x_{n}\right\}$ which converges $p$-adically in $X_{L}(M)$. 
(Note that by choosing any proper model over $\mathcal{O}_{L}$ of $X_{L}$, we obtain a p-adic topology on $X_{L}\left(L^{\mathrm{tm}}\right)$ which is easily seen to be independent of the model chosen.)

By replacing $K$ be a finite extension of $K$, we may make the following simplifying assumptions:

(1) $X_{K}$ has stable reduction. We denote by $\mathcal{X} \rightarrow \operatorname{Spec}\left(\mathcal{O}_{K}\right)$ the unique stable extension of $X_{K}$ over $\mathcal{O}_{K}$. We denote by $\mathcal{J} \rightarrow \operatorname{Spec}\left(\mathcal{O}_{K}\right)$ the unique semi-abelian scheme over $\mathcal{O}_{K}$ whose generic fiber is the Jacobian of $X_{K}$.

(2) $X_{K}(K)$ is nonempty.

Note that replacing $K$ by a finite extension of $K$ does not affect the validity of the convergence assertion $(*)^{\text {con }}$.

Let us fix an $n \geq 1$. Let us consider the morphism $x_{n}: \operatorname{Spec}\left(L^{\mathrm{tm}}\right) \rightarrow X_{K}$. This morphism induces a morphism

$$
\beta_{n}: \Gamma_{L^{\mathrm{tm}}} \rightarrow \Pi_{X_{K}} \rightarrow \Pi_{X_{K}} /\left(\Delta_{X}\right)^{<n>}
$$

which is well-defined up to conjugation by an element of $\Delta_{X}$. Then it follows immediately from the definitions (and the fact that $x_{n}$ arose from an $L^{\mathrm{tm}}$-rational point of $X_{L}^{n}$ ) that:

This morphism $\beta_{n}$ is equal to the composite of $\left.\alpha\right|_{\Gamma_{L} \mathrm{tm}}: \Gamma_{L^{\mathrm{tm}}} \rightarrow \Pi_{X_{K}}$ with the natural projection $\Pi_{X_{K}} \rightarrow \Pi_{X_{K}} /\left(\Delta_{X}\right)^{<n>}$.

Thus, in particular, $\beta_{n}$ is independent of the choice of $\widetilde{x}_{n}$. Moreover, by $(*)^{\text {non }}$, it follows that by taking $n$ to be sufficiently large, we may assume that the restriction of $\beta_{n}$ to $\Gamma_{L^{\mathrm{tm}} \cdot \bar{K}}\left(\subseteq \Gamma_{L^{\mathrm{tm}}}\right)$ is nontrivial. This implies, in particular, that $x_{n}$ does not factor through any finite extension of $K$.

Note that by properness, $x_{n}$ extends to a morphism $\xi_{n}: \operatorname{Spec}\left(\mathcal{O}_{L^{\mathrm{tm}}}\right) \rightarrow \mathcal{X}$. Let $K^{\mathrm{tm}}$ be the algebraic closure of $K$ in $L^{\mathrm{tm}}$. Thus, $K^{\mathrm{tm}}$ is a maximal tamely ramified extension

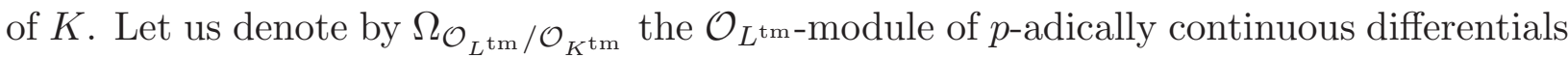
of $\mathcal{O}_{L^{\mathrm{tm}}}$ over $\mathcal{O}_{K^{\mathrm{tm}}}$. Thus, $\Omega_{\mathcal{O}_{L^{\mathrm{tm}}} / \mathcal{O}_{K}^{\mathrm{tm}}}$ is a free $\mathcal{O}_{L^{\mathrm{tm}}}-$ module of rank one.

Lemma 9.1. By differentiating $\xi_{n}$, we obtain a natural morphism $d \xi_{n}: \xi_{n}^{*} \omega_{\mathcal{X} / \mathcal{O}_{K}} \rightarrow$ $\Omega_{\mathcal{O}_{L} \mathrm{tm}} / \mathcal{O}_{K^{\mathrm{tm}}}$.

Proof. Suppose that $x_{n}$ is defined over some finite tamely ramified extension $L^{\prime}$ of $L$. Suppose, moreover, that $L^{\prime}$ contains a finite tamely ramified extension $K^{\prime}$ of $K$ such that $\mathfrak{m}_{K^{\prime}} \cdot \mathcal{O}_{L^{\prime}}=\mathfrak{m}_{L^{\prime}}$. Then, if we equip $\operatorname{Spec}\left(\mathcal{O}_{K}\right), \operatorname{Spec}\left(\mathcal{O}_{K^{\prime}}\right)$ and $\operatorname{Spec}\left(\mathcal{O}_{L^{\prime}}\right)$ with the $\log$ structures defined by the special points, and $\mathcal{X}$ with the log structure whose monoid is the sheaf of functions invertible on the generic fiber, we obtain a log morphism 
$\operatorname{Spec}\left(\mathcal{O}_{L^{\prime}}\right)^{\log } \rightarrow \mathcal{X}^{\log }$ compatible with $\xi_{n}$ and $\operatorname{Spec}\left(\mathcal{O}_{K^{\prime}}\right)^{\log } \rightarrow \operatorname{Spec}\left(\mathcal{O}_{K}\right)^{\log }$. Moreover,

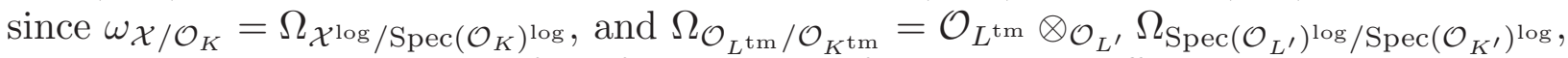
we thus obtain $d \xi_{n}$ by means of the functoriality of logarithmic differentials. The naturality of $d \xi_{n}$ is easily checked by looking at the generic fibers.

Let $\Gamma_{X}^{\Omega} \stackrel{\text { def }}{=} H^{0}\left(\mathcal{X}, \omega_{\mathcal{X} / \mathcal{O}_{K}}\right)$. Then we propose to prove in the present situation that

$(*)^{\text {ind }}$ There exists a number $n_{0}$ depending only on $K$ such that for all $n \geq n_{0}$, the natural morphism $\Gamma_{X}^{\Omega} \rightarrow \Omega_{\mathcal{O}_{L^{\mathrm{tm}}}} / \mathcal{O}_{K^{\mathrm{tm}}} \otimes \mathbf{Z} / p^{n-n_{0}} \mathbf{Z}$ induced by $d \xi_{n}$ is independent of $x_{n}$ (i.e., depends only on $\alpha$ ).

To keep the notation simple, let us note that by replacing $K$ by a tamely ramified extension of $K$, and then replacing $L$ by an unramified extension of $L$, we may assume that $x_{n}$ is, in fact, defined over $L$. (Note that replacing $L$ and $K$ by extensions in this fashion will not affect the validity of $(*)^{\text {ind }}$.) Thus, we shall regard $x_{n}$ and $\xi_{n}$ as morphisms $\operatorname{Spec}(L) \rightarrow X_{K}$ and $\operatorname{Spec}\left(\mathcal{O}_{L}\right) \rightarrow \mathcal{X}$, respectively.

Let $P \stackrel{\text { def }}{=} \mathbf{P}\left(\Gamma_{X}^{\Omega}\right)$. (That is, $P$ is the projective space over $\mathcal{O}_{K}$ defined by $\Gamma_{X}^{\Omega}$.) Then there is (by the definition of $\Gamma_{X}^{\Omega}$ ) a natural finite morphism $\lambda_{K}: X_{K} \rightarrow P_{K}$. Let $\mathcal{N} \rightarrow \operatorname{Spec}\left(\mathcal{O}_{K}\right)$ be the Néron model of $J_{X}$. Thus, $\mathcal{J} \subseteq \mathcal{N}$ is an open subscheme of $\mathcal{N}$. Since $X_{K}(K)$ is nonempty, we can use a $K$-rational point of $X_{K}$ to identify $J_{X}$ with $J_{X}^{(1)}$. Thus, we obtain a morphism $X_{K} \hookrightarrow \mathcal{N}_{K}=J_{X}$. By the defining property of the Néron model, and the fact that $\mathcal{O}_{L}$ is geometrically regular over $\mathcal{O}_{K}$, it follows that composing this morphism with $x_{n}$ gives rise to a morphism $\zeta_{L}: \operatorname{Spec}\left(\mathcal{O}_{L}\right) \rightarrow \mathcal{N}$. Moreover, (after possibly replacing $K$ by a finite unramified extension of $K$ ), we may assume that there exists some $\zeta_{K}: \operatorname{Spec}\left(\mathcal{O}_{K}\right) \rightarrow \mathcal{N}$ such that $\zeta_{K}$ and $\zeta_{L}$ map the special points of $\operatorname{Spec}\left(\mathcal{O}_{K}\right)$ and $\operatorname{Spec}\left(\mathcal{O}_{L}\right)$, respectively, to the same geometric connected component of the special fiber of $\mathcal{N}$. Thus, by translating by $\zeta_{K}$, we may assume that our identification of $J_{X}$ with $J_{X}^{(1)}$ is such that $\zeta_{L}$ maps into $\mathcal{J} \subseteq \mathcal{N}$. We denote the resulting morphism by $j_{n}: \operatorname{Spec}\left(\mathcal{O}_{L}\right) \rightarrow \mathcal{J}$.

Now let us consider the $\Gamma_{K}$-module $H^{1}\left(X_{\bar{K}}, \mathbf{Z}_{p}(1)\right)=H^{1}\left(\mathcal{J}_{\bar{K}}, \mathbf{Z}_{p}(1)\right)$. Here, the identification of $H^{1}\left(X_{\bar{K}}, \mathbf{Z}_{p}(1)\right)$ with $H^{1}\left(\mathcal{J}_{\bar{K}}, \mathbf{Z}_{p}(1)\right)$ is the identification induced by $X_{K} \hookrightarrow$ $J_{X}^{(1)} \cong J_{X}$. This identification is the same as the standard identification since translation by $K$-valued points of $\mathcal{J}$ induces the identity on $H^{1}\left(\mathcal{J}_{\bar{K}}, \mathbf{Z}_{p}(1)\right)$. Let $H^{\mathrm{cb}} \subseteq H^{1}\left(\mathcal{J}_{\bar{K}}, \mathbf{Z}_{p}(1)\right)$ be the maximal $\mathbf{Z}_{p}$-submodule such that $\Gamma_{K}$ acts on $H^{\mathrm{cb}}(-1)$ through a finite, unramified quotient. (Here, "cb" stands for "combinatorial." Note that this inclusion $H^{\mathrm{cb}} \subseteq$ $H^{1}\left(\mathcal{J}_{\bar{K}}, \mathbf{Z}_{p}(1)\right)$ is Cartier-dual to the quotient $H_{X} \rightarrow H_{X} / H_{X}^{F}$ (in the notation of the discussion following Definition 6.3 in Section 6).) By the theory of [FC], Chapter III, we know that $\mathcal{C}_{X} \stackrel{\text { def }}{=} H^{1}\left(\mathcal{J}_{\bar{K}}, \mathbf{Z}_{p}(1)\right) / H^{\text {cb }}$ may be $\left(\Gamma_{K}\right.$-equivariantly $)$ identified with $H^{1}\left(\mathcal{G}_{\bar{K}}, \mathbf{Z}_{p}(1)\right)$ for some semi-abelian scheme $\mathcal{G} \rightarrow \operatorname{Spec}\left(\mathcal{O}_{K}\right)$ which can be represented as an extension

$$
0 \rightarrow \mathcal{T} \rightarrow \mathcal{G} \rightarrow \mathcal{A} \rightarrow 0
$$


of an abelian scheme $\mathcal{A} \rightarrow \operatorname{Spec}\left(\mathcal{O}_{K}\right)$ by a torus $\mathcal{T} \rightarrow \operatorname{Spec}\left(\mathcal{O}_{K}\right)$. Moreover, the $p$-adic completion $\widehat{\mathcal{G}}$ of $\mathcal{G}$ is equal to the $p$-adic completion $\widehat{\mathcal{J}}$ of $\mathcal{J}$. Indeed, relative to the identification $\widehat{\mathcal{G}}=\widehat{\mathcal{J}}$, the identification $\mathcal{C}_{X}=H^{1}\left(\mathcal{G}_{K^{\prime}}, \mathbf{Z}_{p}(1)\right)$ is obtained as follows: Given a finite étale covering $\mathcal{Q}_{\bar{K}} \rightarrow \mathcal{J}_{\bar{K}}$, we let $\mathcal{Q}$ be the normalization of $\mathcal{J}$ in $\mathcal{Q}_{\bar{K}}$, so $\mathcal{Q}$ is finite over $\mathcal{J}$. Thus, by $p$-adically completing, we obtain a formal morphism $\widehat{\mathcal{Q}} \rightarrow \widehat{\mathcal{J}}=\widehat{\mathcal{G}}$ (whose relative differentials are annihilated by a power of $p$ ) which can be algebrized to some finite $\mathcal{R} \rightarrow \mathcal{G}$ (whose relative differentials are annihilated by a power of $p$ ). Thus, $\mathcal{R}_{\bar{K}} \rightarrow \mathcal{G}_{\bar{K}}$ is finite étale. In other words, the correspondence between coverings given by $\mathcal{Q}_{\bar{K}} \mapsto \mathcal{R}_{\bar{K}}$ induces a morphism $H^{1}\left(\mathcal{J}_{\bar{K}}, \mathbf{Z}_{p}(1)\right) \rightarrow H^{1}\left(\mathcal{G}_{\bar{K}}, \mathbf{Z}_{p}(1)\right)$ whose kernel is $H^{\mathrm{cb}}$, and thus allows us to identify $H^{1}\left(\mathcal{G}_{\bar{K}}, \mathbf{Z}_{p}(1)\right)$ with $\mathcal{C}_{X}$.

Now by $p$-adically completing $j_{n}: \operatorname{Spec}\left(\mathcal{O}_{L}\right) \rightarrow \mathcal{J}$, applying $\widehat{\mathcal{G}}=\widehat{\mathcal{J}}$, and then algebrizing, we thus obtain a morphism $g_{n}: \operatorname{Spec}\left(\mathcal{O}_{L}\right) \rightarrow \mathcal{G}$. Moreover, $g_{n}$ induces a morphism on cohomology groups

$$
H^{1}\left(g_{n}\right): H^{1}\left(\mathcal{G}_{\bar{K}}, \mathbf{Z}_{p}(1)\right) \rightarrow H^{1}\left(\Gamma_{L / K}, \mathbf{Z}_{p}(1)\right)
$$

Thus, we have a commutative diagram of $\Gamma_{K}$-modules:

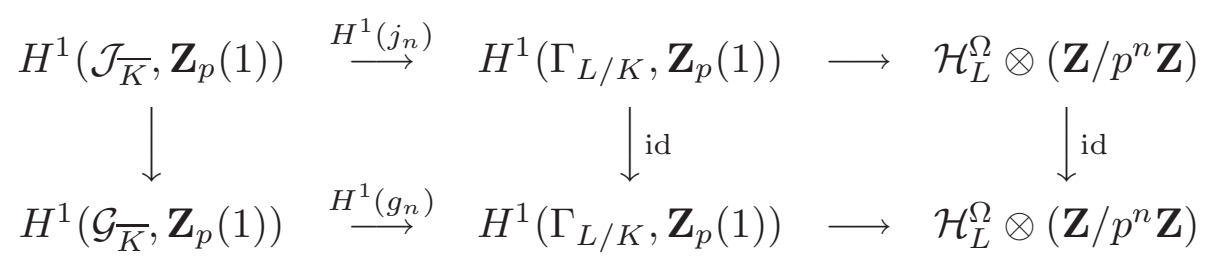

where the vertical morphism on the left is the natural projection discussed in the preceding paragraph, and the horizontal morphisms on the right are the natural ones. Recall that $\mathcal{H}_{L}^{\Omega}$ is, by definition, the quotient of $H^{1}\left(\Gamma_{L / K}, \widehat{\mathcal{O}}_{\bar{L}}(1)\right)$ by its torsion submodule. Now, by sorting through the definitions (cf. the discussion preceding Lemma 9.1), it is clear that the composite morphism on the upper row is completely determined by $\alpha$ (i.e., is independent of the particular choice of $x_{n}$ ). Thus, it follows that the composite morphism on the bottom row gives rise to a morphism

$$
\epsilon_{n}: H^{1}\left(\mathcal{G}_{\bar{K}}, \widehat{\mathcal{O}}_{\bar{K}}(1)\right) \rightarrow \mathcal{H}_{L}^{\Omega} \otimes \mathbf{Z} / p^{n} \mathbf{Z}
$$

which is completely determined by $\alpha$. Moreover, since $\mathcal{H}_{L}^{\Omega}$ is $p$-adically separated (see Consequence $(2)$ of [Falt1] below), it follows (from $(*)^{\text {non }}$ ) that $\epsilon_{n}$ is nonzero for $n$ sufficiently large.

Now, note that $\mathcal{G}$ is a smooth $\mathcal{O}_{K}$-scheme with an obvious compactification $\overline{\mathcal{G}}$ such that $\overline{\mathcal{G}}-\mathcal{G}$ is a divisor with normal crossings. (Indeed, $\mathcal{G}$ is a product of $\mathbf{G}_{m}$-torsors over the $\mathcal{O}_{K}$-proper scheme $\mathcal{A}$, so we simply compactify each of these $\mathbf{G}_{m}$-torsors to a $\mathbf{P}^{1}$-bundle.) In particular, it follows that we can apply the theory of [Falt1], in the case of good reduction. 
Remark. The argument of [Falt1] in the bad reduction case is known to have gaps, which is one reason why we took pains to avoid applying [Falt1] to $\mathcal{J}_{K}$. Another reason is that in the bad reduction case, one only has " $\mathbf{Q}_{p}$-results," not integral results (say, over $\mathbf{Z} / p^{n} \mathbf{Z}$ ), as we need here.

Let $n_{0}^{\prime \prime}$ be an integer such that the $p$-adic valuation of the different of $K$ over $\mathbf{Q}_{p}$ is $\leq$ the $p$-adic valuation of $p^{n_{0}^{\prime \prime}}$. Let $n_{0}^{\prime} \stackrel{\text { def }}{=} n_{0}^{\prime \prime}+\operatorname{ord}_{p}(g !)$. Let $n_{0} \stackrel{\text { def }}{=} n_{0}^{\prime}+2$. The consequences of the theory of [Falt1] that we use are as follows:

(1) The $\Gamma_{K}$-module $H^{1}\left(\mathcal{G}_{\bar{K}}, \bar{K}^{\wedge}\right)$ is Hodge-Tate. Let us denote by $\mathcal{H}_{\mathcal{G}}^{\Omega}$ the quotient of $H^{1}\left(\mathcal{G}_{\bar{K}}, \widehat{\mathcal{O}}_{\bar{K}}(1)\right)$ by the submodule that is contained in the portion of $H^{1}\left(\mathcal{G}_{\bar{K}}, \bar{K}^{\wedge}(1)\right)$ of weight 1. Thus, $\mathcal{H}_{\mathcal{G}}^{\Omega} \otimes_{\mathbf{z}_{p}} \mathbf{Q}_{p}$ is of weight 0 . Let $\Omega_{\mathcal{G}}^{\text {inv }}$ be the $\mathcal{O}_{K}$-module of invariant differentials (over $\mathcal{O}_{K}$ ) on the group scheme $\mathcal{G}$. Then there is a natural $\Gamma_{K}$-equivariant morphism (Theorems 2.4 and 3.1 of II. of [Falt1])

$$
\Omega_{\mathcal{G}}^{\mathrm{inv}} \otimes \mathcal{O}_{K}\left\{\left(p^{n_{0}} \cdot \mathcal{O}_{\bar{K}}\right) /\left(p^{n_{0}+n} \cdot \mathcal{O}_{\bar{K}}\right)\right\} \rightarrow \mathcal{H}_{\mathcal{G}}^{\Omega} \otimes \mathbf{Z} / p^{n} \mathbf{Z}
$$

with an inverse "up to a factor of $p^{n_{0}}$." Here the point of the $n_{0}$ is to take care of the " $g ! \cdot \rho$ " of [Falt1]: the $2=1+1$ added to $n_{0}^{\prime}$ is to take care of the $\frac{1}{p-1} \leq 1$ that always arises, plus the additional $\frac{e-1}{e}<1$ that arise from the tame ramification which we allow (see [Fo], Theorem 1' for more details on the computation of [Falt1]'s " $\rho$ ").

(2) $\mathcal{H}_{L}^{\Omega}$ is $p$-adically separated. Moreover, there is a natural $\Gamma_{K}$-equivariant morphism (Theorem 4.2 of I. of [Falt1])

$$
\Omega_{\mathcal{O}_{L} / \mathcal{O}_{K}} \otimes \mathcal{O}_{K}\left\{\left(p^{n_{0}} \cdot \mathcal{O}_{\bar{K}}\right) /\left(p^{n_{0}+n} \cdot \mathcal{O}_{\bar{K}}\right)\right\} \rightarrow \mathcal{H}_{L}^{\Omega} \otimes \mathbf{Z} / p^{n} \mathbf{Z}
$$

with an inverse "up to a factor of $p^{n_{0}}$." In particular, by considering weights, it follows that $\epsilon_{n}$ factors through the quotient $\mathcal{H}_{\mathcal{G}}^{\Omega}$ of $H^{1}\left(\mathcal{G}_{\bar{K}}, \widehat{\mathcal{O}}_{\bar{K}}(1)\right)$.

(3) The morphisms (and inverses up to a factor) of (1) and (2) are compatible with each other, $\epsilon_{n}$, and the natural morphism $\Omega_{\mathcal{G}}^{\text {inv }} \rightarrow \Omega_{\mathcal{O}_{L} / \mathcal{O}_{K}}$ induced by differentiating $g_{n}$. That is to say, we have a commutative diagram:

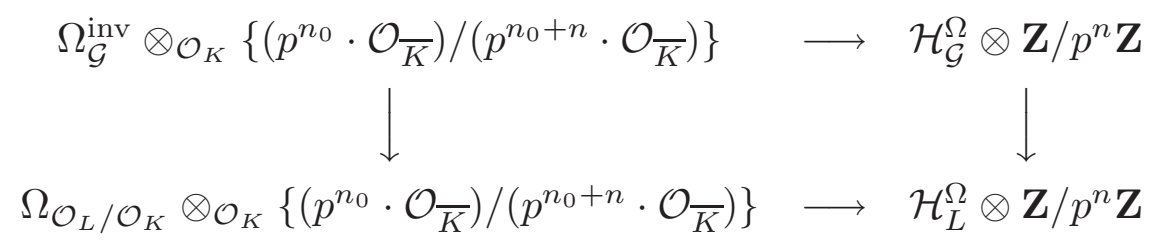


(where the horizontal morphisms are the morphisms of (1) and (2), respectively, the vertical morphism on the left is induced by differentiating $g_{n}$, and the vertical morphism on the right is induced by $\epsilon_{n}$ ).

These assertions allow us to immediately conclude $(*)^{\text {ind }}$, as follows: Since $\epsilon_{n}$ is determined by $\alpha$, it thus follows that the morphism (induced by differentiating $g_{n}$ ) $\Omega_{\mathcal{G}}^{\text {inv }} \rightarrow \Omega_{\mathcal{O}_{L} / \mathcal{O}_{K}} \otimes$ $\left(\mathbf{Z} / p^{n-n_{0}} \mathbf{Z}\right)$ is determined by $\alpha$. On the other hand, $\Omega_{\mathcal{G}}^{\text {inv }}$ can naturally be identified with $\Omega_{\mathcal{J}}^{\text {inv }}$. Moreover, $\Omega_{\mathcal{J}}^{\text {inv }}$ can naturally be identified with $\Gamma_{X}^{\Omega}$. This completes the proof of $(*)^{\text {ind }}$.

Lemma 9.2. $\quad$ For $n$ sufficiently large, the morphism $\mathcal{H}_{\mathcal{G}}^{\Omega} \rightarrow \mathcal{H}_{L}^{\Omega} \otimes\left(\mathbf{Z} / p^{n} \mathbf{Z}\right)$ induced by $\epsilon_{n}$ is nonzero.

Proof. This follows formally from $(*)^{\text {non }}$.

Now we are ready to tackle the convergence assertion $(*)^{\text {con }}$. Thus, we go back to working over $L^{\mathrm{tm}}$ and $K^{\mathrm{tm}}$. In particular, by composing $x_{n}$ with $\lambda_{K}$ and applying the valuative criterion for properness, we obtain a morphism $\lambda_{n}: \operatorname{Spec}\left(\mathcal{O}_{L^{\mathrm{tm}}}\right) \rightarrow P$. Moreover, this $\mathcal{O}_{L^{\mathrm{tm}}}$-valued point $\lambda_{n}$ of the projective space $P$ is precisely the point defined by the morphism $\Omega_{\mathcal{G}}^{\text {inv }} \rightarrow \Omega_{\mathcal{O}_{L}} / \mathcal{O}_{K} \otimes_{\mathcal{O}_{L}} \mathcal{O}_{L^{\mathrm{tm}}}=\Omega_{\mathcal{O}_{L^{\mathrm{tm}}} / \mathcal{O}_{K}^{\mathrm{tm}}}$ (obtained by differentiating $g_{n}$ ). (Note that here we use the fact remarked above that $\Omega_{\mathcal{O}_{L} \mathrm{tm}} / \mathcal{O}_{K^{\mathrm{tm}}}$ is a free $\mathcal{O}_{L^{\mathrm{tm}}}$-module of rank one.) Thus, $(*)^{\text {ind }}$ and Lemma 9.2 imply that the points $\lambda_{n}$ of $P\left(\mathcal{O}_{L^{\mathrm{tm}}}\right)$ converge $p$-adically to a point $\lambda_{\infty} \in P\left(\widehat{\mathcal{O}}_{L^{\mathrm{tm}}}\right)=P(M)$. On the other hand, by "Krasner's Lemma" (see, e.g., [Kobl], p. 70), since $\lambda_{K}: X_{K} \rightarrow P_{K}$ is finite, it follows that some subsequence $\left\{x_{n_{i}}\right\}$ of $\left\{x_{n}\right\}$ converges to a point $x_{\infty} \in X_{K}(M)$. This proves the assertion $(*)^{\text {con }}$, as desired.

In fact, we can say more. For $m \geq 1$, let $\widetilde{x}_{n}^{m}$ be the image of $\widetilde{x}_{n+m}$ in $X_{L}^{m}\left(L^{\mathrm{tm}}\right)$ under the natural morphism $X_{L}^{n+m} \rightarrow X_{L}^{m}$. Thus, we obtain a sequence $\left\{\widetilde{x}_{n}^{m}\right\}$ (in the index $n$ ) of points of $X_{L}^{m}\left(L^{\mathrm{tm}}\right)$. Since $X_{L}^{m} \rightarrow X_{L}$ is finite, then by appying "Krasner's Lemma" again, see that a subsequence of the sequence $\left\{\widetilde{x}_{n}^{m}\right\}$ converges $p$-adically in $X_{L}^{m}(M)$. Moreover, by the process of "Cantor diagonalization" (of elementary analysis), we thus see that we have proven the following key result (which is the main result of this Section):

Lemma 9.3. We assume notation as in the first paragraph of this Section. Suppose that we have a sequence of points $\left\{\widetilde{x}_{n}\right\}$, where $\widetilde{x}_{n} \in X_{L}^{n}\left(L^{\mathrm{tm}}\right)$. Then there exists a subsequence $\left\{\widetilde{x}_{n_{i}}\right\}$ of this sequence with the following property: For each $m \geq 0$, the sequence obtained by projecting those $\widetilde{x}_{n_{i}}$ with $n_{i} \geq m$ to $X_{L}^{m}(M)$ converges to some $\widetilde{x}_{\infty}^{m} \in X_{L}^{m}(M)$. In particular, $X_{L}^{\infty}(M)$ is nonempty.

Proof. The last assertion is proven by noting that the $\widetilde{x}_{\infty}^{m} \in X_{L}^{m}(M)$ form a compatible system, hence define a point of $\widetilde{x}_{\infty}^{\infty} \in X_{L}^{\infty}(M)$. 
Finally, before proceeding, we make the following technically trivial, but important observation: Since the arithmetic fundamental group of $X_{L}^{\infty}$ is just $\Gamma_{L}$ (i.e., $\operatorname{Im}\left(\alpha_{s}\right)$ ), it follows that the morphism

$$
\Gamma_{M} \rightarrow \Pi_{X_{K}}
$$

induced on fundamental groups by the image in $X_{K}(M)$ of any point (e.g., $\left.\widetilde{x}_{\infty}^{\infty}\right)$ of $X_{L}^{\infty}(M)$ is none other than the restriction of $\alpha: \Gamma_{L} \rightarrow \Pi_{X_{K}}$ to $\Gamma_{M}$.

\section{Section 10: Uniqueness and Rationality of the Limit Point}

We continue with the notation of the preceding Section. In this Section, we would like to show that the point $\widetilde{x}_{\infty}^{\infty} \in X_{L}^{\infty}(M)$ constructed in Lemma 9.3 is the unique element of $X_{L}^{\infty}(M)$, and that it in fact descends to a point of $X_{L}^{\infty}(L)$. We remark that much of the material of this Section is not absolutely logically necessary for the proof of the main results of this paper, but is included partly for aesthetic reasons, and partly because its inclusion (in the opinion of the author) makes the proof of the main results of the paper more transparent.

First, let us make the trivial observation that any point in $X_{L}^{\infty}(M)$ arises as a $\widetilde{x}_{\infty}^{\infty}$ (as in Lemma 9.3) for some sequence of $\widetilde{x}_{n} \in X_{L}^{n}\left(L^{\mathrm{tm}}\right)$. (Indeed, this follows by using the fact that $M=\left(L^{\mathrm{tm}}\right)^{\wedge}$ to approximate the images of the given point in the various $X_{L}^{n}(M)^{\prime}$ 's.) Thus, without loss of generality, we can apply the results of the discussion of Section 9 to our analysis of an arbitrary point of $X_{L}^{\infty}(M)$.

Now recall the limit point $\lambda_{\infty} \in P(M)$ of the discussion following Lemma 9.2. This limit point is clearly independent of the choice of the particular sequence $\left\{\widetilde{x}_{n}\right\}$ under consideration - i.e., it depends only on $\alpha$. More precisely, $\lambda_{\infty} \in P(M) \subseteq P\left(\bar{L}^{\wedge}\right)$ is the point defined by the surjection

$$
\begin{aligned}
\Gamma_{X}^{\Omega} \otimes_{\mathcal{O}_{K}} \bar{L}^{\wedge} & =\Omega_{\mathcal{G}}^{\mathrm{inv}} \otimes_{\mathcal{O}_{K}} \bar{L}^{\wedge} \\
& =\mathcal{H}_{\mathcal{G}}^{\Omega} \otimes_{\widehat{\mathcal{O}}_{\bar{K}}} \bar{L}^{\wedge} \rightarrow \mathcal{H}_{L}^{\Omega} \otimes_{\mathbf{z}_{p}} \mathbf{Q}_{p}=\Omega_{\mathcal{O}_{L} / \mathcal{O}_{K}} \otimes_{\mathcal{O}_{L}} \bar{L}^{\wedge}
\end{aligned}
$$

induced by $\alpha$ (cf. the discussion preceding Lemma 9.2). Note that $\Gamma_{L}$ acts naturally on all of these modules, and that this surjection is $\Gamma_{L}$-equivariant. Indeed, this follows by transport of structure from the fact that $\alpha$ is defined on $\Gamma_{L}$, not just on $\Gamma_{L^{\mathrm{tm}} \cdot \bar{K}}$. Thus, we conclude the following

Lemma 10.1. The image $\lambda_{\infty} \in P(M)$ of $\widetilde{x}_{\infty}^{\infty} \in X_{L}^{\infty}(M)$ under the natural morphism $X_{L}^{\infty} \rightarrow X_{K} \rightarrow P_{K}$ is independent of $\widetilde{x}_{\infty}^{\infty}$ and, moreover, $\lambda_{\infty}$ is defined over $L$. 
The next step is to observe that this argument can be modified so as to show that the image of $\widetilde{x}_{\infty}^{\infty}$ in the projective space defined by the differentials on $X_{L}^{n}$ (for $n \geq 1$ ) is independent of $\widetilde{x}_{\infty}^{\infty}$ and defined over $L$. To see this, we need to introduce some new objects. First, let us fix $n$. Now observe that (after possibly enlarging $K$ ) there exists a finite étale covering $Z_{K}^{n} \rightarrow \operatorname{Spec}(K)$ of $X_{K}$ with stable reduction $\mathcal{Z}^{n} \rightarrow \operatorname{Spec}\left(\mathcal{O}_{K}\right)$, together with a finite Galois extension $\widetilde{L}$ of $L$ such that

$$
X_{\widetilde{L}}^{n}=X_{L}^{n} \otimes_{L} \widetilde{L} \cong Z_{\widetilde{L}}^{n}=Z_{K}^{n} \otimes_{K} \widetilde{L}
$$

Now let $m \geq n$, and consider $\widetilde{x}_{m} \in X_{L}^{m}\left(L^{\mathrm{tm}}\right) \subseteq X_{L}^{m}\left(\widetilde{L}^{\mathrm{tm}}\right)$, where $\widetilde{L}^{\mathrm{tm}}$ is the composite of $L^{\mathrm{tm}}$ and $\widetilde{L}$ (as extensions of $L$ ). (Note that $\widetilde{L}^{\mathrm{tm}}$ is then a maximal tamely ramified extension of $\widetilde{L}$.) Thus, by projecting $\widetilde{x}_{m}$ to a point of $Z_{K}^{n}$, we obtain a morphism

$$
z_{m}: \operatorname{Spec}\left(\mathcal{O}_{\widetilde{L}^{\mathrm{tm}}}\right) \rightarrow \mathcal{Z}^{n}
$$

Let us assume that we have chosen $K$ large enough so that $Z_{K}^{n}(K)$ is nonempty, so that we can identify $J_{Z_{K}^{n}}$ (i.e., the Jacobian of $Z_{K}^{n}$ ) with $J_{Z_{K}^{n}}^{(1)}$. Let $\mathcal{N}_{Z_{K}^{n}} \rightarrow \operatorname{Spec}\left(\mathcal{O}_{K}\right)$ be the Néron model of $Z_{K}^{n}$ over $\mathcal{O}_{K}$. More generally, in the following discussion we shall denote by $\mathcal{N}_{(-)}$the Néron model of any proper hyperbolic curve "(-)" over a discretely valued field. In fact, we shall even use this notation for proper hyperbolic curves over inductive limits of discretely valued fields in which case this notation is to be taken to mean the corresponding inductive limit of the Néron models. Also, we shall denote by

\section{$\operatorname{Comp}\left(\mathcal{N}_{(-)}\right)$}

the (inductive limit of) finite abelian (étale) group scheme(s) of connected components of the special fiber of the Néron model $\mathcal{N}_{(-)}$.

The main technical difficulty that we must overcome in order to apply the argument of Section 9 (for $X_{K}$ ) to the curves $Z_{K}^{n}$ is the following: The points that we are interested in are the points $z_{m}$ of $Z_{K}^{n}\left(\widetilde{L}^{\mathrm{tm}}\right)=\mathcal{Z}^{n}\left(\mathcal{O}_{\widetilde{L}^{\mathrm{tm}}}\right)$. These points define points of $J_{Z_{K}^{n}}^{(1)} \cong J_{Z_{K}^{n}}$, hence points

$$
z_{m}^{\mathcal{N}}: \operatorname{Spec}\left(\mathcal{O}_{\widetilde{L}^{\mathrm{tm}}}\right) \rightarrow \mathcal{N}_{Z_{L^{\mathrm{tm}}}^{n}}
$$

Then, in order to apply the argument of Section 9, we must show that:

(After replacing $K$ by a finite extension of $K$ which is independent of $m), z_{m}^{\mathcal{N}}$ maps the special point of $\operatorname{Spec}\left(\mathcal{O}_{\widetilde{L}^{\mathrm{tm}}}\right)$ to the same component of $\operatorname{Comp}\left(\mathcal{N}_{Z_{L^{\mathrm{tm}}}^{n}}\right)$ as some $K^{\mathrm{tm}}$-valued point of $\mathcal{N}_{Z_{L^{\mathrm{tm}}}^{n}}$. 
(see Lemma 10.2 below for an alternate formulation.) To show this, we reason as follows: Let us write $\widetilde{L}^{\text {unr }}$ for the maximal unramified extension of $\widetilde{L}$ in $\widetilde{L}^{\mathrm{tm}}$. Thus, $\widetilde{L}^{\mathrm{tm}}$ is a totally tamely ramified extension of $\widetilde{L}^{\text {unr }}$. In particular, $\widetilde{L}^{\mathrm{tm}}$ is a union of finite Galois extensions of $\widetilde{L}^{\text {unr }}$ which are of degree (over $\widetilde{L}^{\text {unr }}$ ) prime to $p$. In particular, it follows that the cokernel of the inclusion

$$
\operatorname{Comp}\left(\mathcal{N}_{Z_{L^{\mathrm{unr}}}^{n}}\right) \hookrightarrow \operatorname{Comp}\left(\mathcal{N}_{Z_{L^{\mathrm{tm}}}^{n}}\right)
$$

is annihilated by integers prime to $p$. (Indeed, this follows by applying the "trace map" (for subextensions of $\widetilde{L}^{\mathrm{tm}}$ over $\widetilde{L}^{\text {unr }}$ ) to the second "Comp," and observing that (since $\operatorname{Gal}\left(\widetilde{L}^{\mathrm{tm}} / \widetilde{L}^{\mathrm{unr}}\right)$ clearly acts trivially on $\left.\operatorname{Comp}\left(\mathcal{N}_{Z_{L^{\mathrm{tm}}}^{n}}\right)\right)$ this trace map is just multiplication by some integer prime to $p$.) On the other hand, since $\widetilde{L}^{\text {unr }}$ is an unramified extension of $\widetilde{L}$, it follows (essentially from the definition of the Néron model) that $\mathcal{N}_{Z_{L}^{n}}=\mathcal{N}_{Z_{L}^{n}}$. In particular, since $\widetilde{L}$ is a discretely valued field, it follows that $\operatorname{Comp}\left(\mathcal{N}_{Z_{\widetilde{L}}^{n}}\right)$ is finite. In other words, we conclude that: the p-torsion of $\operatorname{Comp}\left(\mathcal{N}_{Z_{L^{t m}}^{n}}\right)$ is annihilated by some (finite) power of $p$. We are now ready to prove the following Lemma:

Lemma 10.2. After possibly enlarging $K$ by a larger field that is independent of $m$, we may arrange that the morphism $z_{m}^{J}: \operatorname{Spec}\left(\widetilde{L}^{\mathrm{tm}}\right) \rightarrow J_{Z_{K}^{n}}^{(1)} \cong J_{Z_{K}^{n}}$ induced by $z_{m}$ extends to a morphism $\operatorname{Spec}\left(\mathcal{O}_{\widetilde{L}^{\mathrm{tm}}}\right) \rightarrow \mathcal{N}_{Z_{K^{\mathrm{tm}}}^{n}}$, for all $m \geq n$.

Proof. Indeed, without enlarging $K$, it follows from our observation above concerning the $p$-torsion of $\operatorname{Comp}\left(\mathcal{N}_{Z_{L^{\mathrm{tm}}}^{n}}\right)$ that $a \cdot z_{m}^{J}$ extends to $\left(z^{\mathcal{N}}\right)_{m}^{\prime}: \operatorname{Spec}\left(\mathcal{O}_{\widetilde{L}^{\mathrm{tm}}}\right) \rightarrow \mathcal{N}_{Z_{K}^{n}}$ for some positive integer $a$ whose order at the prime $p$ is independent of $m$. Now let us assume (by enlarging $K$-independently of $m$ ) that all of the $p$-torsion points of $J_{Z_{K}^{n}}$ annihilated by $a$ are rational over $K$. Since all prime-to- $p$ torsion points of $J_{Z_{K}^{n}}$ are rational over $K^{\mathrm{tm}}$, it thus follows that the morphism "multiplication by a" on $\mathcal{N}_{Z_{K}^{n}}^{\mathrm{tm}}$ is finite over a neighborhood of the image of $\left(z^{\mathcal{N}}\right)_{m}^{\prime} \otimes_{\mathcal{O}_{K}} \mathcal{O}_{K^{\mathrm{tm}}}$ in $\mathcal{N}_{\mathcal{Z}_{K}^{n}} \otimes_{\mathcal{O}_{K}} \mathcal{O}_{K^{\mathrm{tm}}} \subseteq \mathcal{N}_{\mathcal{Z}_{K}^{\mathrm{tm}}}^{\prime}$. Since $\left(z^{\mathcal{N}}\right)_{m}^{\prime}$ was constructed by multiplying $z_{m}^{J}$ by $a$, it thus follows that $z_{m}^{J}$ extends to a morphism $\operatorname{Spec}\left(\mathcal{O}_{\widetilde{L}^{\mathrm{tm}}}\right) \rightarrow \mathcal{N}_{Z_{K^{\mathrm{tm}}}^{n}}$, as desired.

Now that we have Lemma 10.2, we can use the extended morphism of Lemma 10.2 to construct the analogue of the morphism " $g_{n}$ " of Section 9 (over some tamely ramified extension of $K$ ). Then the rest of the argument of Section 9 goes through without difficulty. More precisely:

Let $\Gamma_{Z^{n}}^{\Omega} \stackrel{\text { def }}{=} H^{0}\left(\mathcal{Z}^{n}, \omega_{\mathcal{Z}^{n}} / \mathcal{O}_{K}\right)$. Let $P^{n} \stackrel{\text { def }}{=} \mathbf{P}\left(\Gamma_{Z^{n}}^{\Omega}\right)$. (That is, $P^{n}$ is the projective space over $\mathcal{O}_{K}$ defined by $\Gamma_{Z^{n}}^{\Omega}$.) Then there is (by the definition of $\Gamma_{Z^{n}}^{\Omega}$ ) a natural finite morphism $\lambda_{K}^{n}: Z_{K}^{n} \rightarrow P_{K}^{n}$. Let $\lambda_{m}^{n}: \operatorname{Spec}\left(\mathcal{O}_{\widetilde{L}^{\mathrm{tm}}}\right) \rightarrow P^{n}$ be the morphism obtained by composing $z_{m}$ with $\lambda_{K}^{n}$. Then, just as in Section 9, one sees via $p$-adic Hodge theory that as $m \rightarrow \infty$ (and 
$n$ remains fixed $)$, the $\lambda_{m}^{n}$ converge to a point in $\lambda_{\infty}^{n} \in P^{n}\left(\widehat{\mathcal{O}}_{\widetilde{L}^{\mathrm{tm}}}\right)=P^{n}\left(\left(\widetilde{L}^{\mathrm{tm}}\right)^{\wedge}\right)$. Moreover, $\lambda_{\infty}^{n}$ is independent of the original sequence $\left\{\widetilde{x}_{m}\right\}$. Finally, just as we saw for Lemma 10.1, by transport of structure and the fact that $\alpha$ (and, here, $X_{L}^{n}$ ) are defined over $L$, it follows that if we regard $\lambda_{\infty}^{n}$ as a point of $P^{X^{n}}\left(\left(\widetilde{L}^{\mathrm{tm}}\right)^{\wedge}\right)$, where $P^{X^{n}}$ is the projective space over $L$ associated to

$$
H^{0}\left(X_{L}^{n}, \omega_{X_{L}^{n} / L}\right)
$$

(for the original $L$ ), then $\lambda_{\infty}^{n} \in P^{X^{n}}(L)$. Thus, we see that we obtain the following analogue of Lemma 10.1:

Lemma 10.3. The image $\lambda_{\infty}^{n} \in P^{X^{n}}(M)$ of $\widetilde{x}_{\infty}^{\infty} \in X_{L}^{\infty}(M)$ under the natural morphism $X_{L}^{\infty} \rightarrow X_{L}^{n} \rightarrow P^{X^{n}}$ is independent of $\widetilde{x}_{\infty}^{\infty}$ and, moreover, $\lambda_{\infty}^{n}$ is defined over $L$.

Now let us recall some basic facts on hyperelliptic curves:

Lemma 10.4. Let $Q$ be a proper hyperbolic curve over an algebraically closed field $\Omega$ of characteristic zero. Then:

(1) If $Q$ is hyperelliptic (i.e., admits a " $g_{2}^{1}$," or linear system of dimension 1 and degree 2), then the $g_{2}^{1}$ is unique.

(2) If $Q$ is non-hyperelliptic, then the canonical morphism from $Q$ into the projective space associated to $H^{0}\left(Q, \omega_{Q / \Omega}\right)$ is an embedding.

(3) Suppose that $W \rightarrow Q$ is a finite étale covering, where $W$ is connected. Then if $Q$ is non-hyperelliptic, so is $W$.

(4) Suppose that $W \rightarrow Q$ (where $W$ is connected) is a cyclic étale covering of degree $m>2$. Then $W$ is non-hyperelliptic.

Thus, in particular, none of the $X_{L}^{n}$ is hyperelliptic, for $n \geq 2$.

Proof. For proofs of assertions (1) and (2), we refer to [Harts], Chapter IV, §5, Propositions 5.2 and 5.3. As for (3), the push-forward of a $g_{2}^{1}$ on $W$ via $W \rightarrow Q$ is a $g_{2}^{1}$ on $Q$, so (3) follows immediately. Now let us consider assertion (4). Let $\sigma$ be a generator of the Galois group of $W$ over $Q$. If $W$ admits a $g_{2}^{1}$, it is unique, hence stabilized by $\sigma$. But this means that there exists some rational function $f_{W}$ on $W$ in this $g_{2}^{1}$ which satisfies $\sigma^{-1}\left(f_{W}\right)=T\left(f_{W}\right)$, where $T$ is some linear fractional transformation with coefficients in $\Omega$. Note that since $\sigma$ has finite order, so does $T$. Thus, if we diagonalize $T$ (by choosing a different $\left.f_{W}\right)$, we may assume that $\sigma^{-1}\left(f_{W}\right)=\lambda \cdot f_{W}$, for some nonzero $\lambda \in \Omega$. (Note that $T$ cannot be parabolic (i.e., a transformation of the form $f_{W} \mapsto f_{W}+\lambda$, for some nonzero $\lambda \in \Omega$ ) since $T$ is of finite order and $\Omega$ is of characteristic zero.) Thus, $f_{W}^{m}$ (which 
is a constant multiple of the norm (relative to $W \rightarrow Q$ ) of $f_{W}$ ) defines a rational function $f_{Q}$ on $Q$. Now $f_{Q}$ is contained in some $g_{2}^{1}$ on $Q$, so its zeroes have order equal to 1 or 2 . On the other hand, $f_{Q}$ has an $m^{\text {th }}$ root in the function field of $W$, so it follows from the assumption that $m>2$ that the covering $W \rightarrow Q$ must be ramified at the zeroes of $f_{Q}$, which is absurd. This contradiction completes the proof.

We summarize our efforts in Sections 9 and 10 as follows:

Corollary 10.5. Let $M$ be the p-adic completion of a maximal tamely ramified extension $L^{\mathrm{tm}}$ of $L$. Suppose that we are given a nondegenerate $\alpha: \Gamma_{L} \rightarrow \Pi_{X_{K}}$, which thus gives rise to $X_{L}^{n}, X_{L}^{\infty}$. Suppose that for each $n \geq 0, X_{L}^{n}\left(L^{\mathrm{tm}}\right)$ is nonempty. Then it follows that the set $X_{L}^{\infty}(M)$ consists of precisely one point, which is, in fact, contained in $X_{L}^{\infty}(L) \subseteq$ $X_{L}^{\infty}(M)$.

In particular, there exists a unique L-valued point of $\operatorname{Spec}(L) \rightarrow X_{K}$ whose induced morphism on fundamental groups (for an appropriate choice of base-points) is the morphism $\alpha: \Gamma_{L} \rightarrow \Pi_{X_{K}}$.

Proof. Indeed, if $\widetilde{x}_{\infty}^{\infty} \in X_{L}^{\infty}(M)$, then by Lemma 10.4, if $n \geq 2$, the image of $\widetilde{x}_{\infty}^{\infty}$ in $X_{L}^{n}(M)$ is determined by the image $\lambda_{\infty}^{n}$ of $\widetilde{x}_{\infty}^{\infty}$ in $P^{X^{n}}(M)$. But, by Lemma $10.3, \lambda_{\infty}^{n}$ is independent of $\widetilde{x}_{\infty}^{\infty}$ and defined over $L$. Thus, it follows that the image of $\widetilde{x}_{\infty}^{\infty}$ in $X_{L}^{n}(M)$ (for $n \geq 2$ ) is independent of $\widetilde{x}_{\infty}^{\infty}$ and defined over $L$. But, by the definition of $X_{L}^{\infty}$, this means that $\widetilde{x}_{\infty}^{\infty}$ itself is "independent of $\widetilde{x}_{\infty}^{\infty}$ " and defined over $L$. (In other words, $X_{L}^{\infty}(M)$ consists of precisely one point, which is, in fact, defined over L.) The last sentence is a formal consequence of the rest of the Corollary. This completes the proof.

\section{Section 11: Hodge-Tate Representations of Infinite Rank}

Let $K$ be a finite extension of $\mathbf{Q}_{p}$. Let $X_{K} \rightarrow \operatorname{Spec}(K)$ be a proper hyperbolic curve over $K$. Let $U_{K}$ be its generic point. Let $H_{U}$ be the abelianization of $\Delta_{U}$. Thus, $H_{U}$ is a $\mathbf{Z}_{p}$-flat topological $\Gamma_{K}$-module of infinite rank. Note that we have a natural surjection $H_{U} \rightarrow H_{X} \rightarrow H_{X}^{I}$ (cf. the discussion following Definition 6.3 for the definition of $H_{X}^{I}$ ). Let $H_{U}^{M} \subseteq H_{U}$ be the kernel of this surjection. Note that the inertia groups (defined by closed points of $X_{K}$ ) of $\Delta_{U}$ all map into $H_{U}^{M}$. Let $H_{U}^{P} \subseteq H_{U}^{M}$ denote the closure of the image of all these inertia groups. (Here the "P" stands for "points.") Thus, $H_{U} / H_{U}^{P}$ may be identified with $H_{X}$, hence is of finite rank over $\mathbf{Z}_{p}$. In this Section, we would like to analyze $H_{U}$, and, in particular, $H_{U}^{P}$ in greater detail.

For simplicity, let us assume (by replacing $K$ by a finite extension of $K$ ) that $X_{K}$ has stable reduction over $\mathcal{O}_{K}$, and that there exists a $K$-valued point $x \in X_{K}(K)$. Let $\wp \in X_{K}$ be a closed point of $X_{K}$. Then there are finitely many points $y_{1}, \ldots, y_{r} \in$ 
$X_{K}(\bar{K})=\operatorname{Hom}\left(\operatorname{Spec}(\bar{K}), X_{K}\right)$ that map to $\wp$. For $i=1, \ldots, r$, let $I_{y_{i}} \subseteq \Delta_{U}$ denote "the" inertia group (well-defined up to conjugation by an element of $\Delta_{U}$ ) associated to $y_{i}$. Thus, projection to the quotient $\Delta_{U} \rightarrow H_{U}$ yields a map

$$
I_{\wp} \stackrel{\text { def }}{=} \bigoplus_{i=1}^{r} I_{y_{i}} \rightarrow H_{U}^{P}
$$

Note, moreover, that $I_{\wp}$ has a natural structure of $\Gamma_{K}$-module (given, for instance, by conjugating by the image of a section $\Gamma_{K} \rightarrow \Pi_{U_{K}}$ (cf. Definition 5.1) induced by $x \in$ $X_{K}(K)$ - note, however, that the $\Gamma_{K}$-action is independent of the choice of $x$ ). Relative to this $\Gamma_{K}$-action on $I_{\wp}$, the above morphism is $\Gamma_{K}$-equivariant. Moreover, by letting $\wp$ range over all closed points of $X_{K}$ other than that defined by $x$, we obtain a continuous $\Gamma_{K}$-equivariant morphism

$$
\Xi: M_{x} \stackrel{\text { def }}{=} \prod_{\wp \neq x} I_{\wp} \rightarrow H_{U}^{P}
$$

In fact,

Lemma 11.1. The morphism $\Xi$ is an isomorphism.

Proof. Note that $\Pi_{U_{K}}$ is equal to the inverse limit of $\Pi_{V_{K}}$, where the limit is taken over all open subsets $V_{K} \subseteq X_{K}-\{x\}$. For such a $V_{K}$, we can define $H_{V}^{P}$ just as we defined $H_{U}^{P}$, and it is well-known that $H_{V}^{P}$ is the direct sum of the $I_{\wp}$, where the sum is taken over all $\wp \in X_{K}-\{x\}-V_{K}$ - indeed, this follows from the well-known (from elementary algebraic topology) structure of the homology group of a Riemann surface obtained by removing a finite number of points from a compact Riemann surface. Passing to the limit proves the Lemma.

Next, we would like to define a quotient $I_{\wp} \rightarrow I_{\wp}^{T}$ as follows: Note that if $K_{\wp}$ is the residue field of $X_{K}$ at $\wp$, then the $\Gamma_{K}$-module $I_{\wp}(-1)$ (where the "(-1)" is a Tate twist) may be naturally identified with $\mathbf{Z}_{p}\left[\operatorname{Hom}_{K}\left(K_{\wp}, \bar{K}\right)\right]$. (Here, $\mathbf{Z}_{p}$ [a set] denotes the free $\mathbf{Z}_{p^{-}}$ module generated by the elements of the set.) Thus, there exists a unique nonzero quotient $I_{\wp}(-1) \rightarrow Q_{\wp}$ stabilized by $\Gamma_{K}$ such that $\Gamma_{K}$ acts trivially on $Q_{\wp}$. Moreover, $Q_{\wp}$ is a free $\mathbf{Z}_{p}$-module of rank one. Let $I_{\wp} \rightarrow I_{\wp}^{T}$ be the quotient obtained by tensoring $I_{\wp}(-1) \rightarrow Q_{\wp}$ with $\mathbf{Z}_{p}(1)$. Moreover, by taking the product of these quotients, we obtain a quotient

$$
M_{x} \rightarrow M_{x}^{T} \stackrel{\text { def }}{=} \prod_{\wp \neq x} I_{\wp}^{T}
$$

Let $H_{U}^{P} \rightarrow H_{U}^{T}$ be the quotient corresponding to this quotient under the isomorphism $\Xi$. Thus, the $\Gamma_{K}$-action on $H_{U}^{T}(-1)$ is trivial. Moreover, $H_{U}^{P} \rightarrow H_{U}^{T}$ has the following universal property: 
Lemma 11.2. Let $N$ be a $\mathbf{Z}_{p}$-flat topological $\Gamma_{K}$-module such that $\Gamma_{K}$ acts trivially on $N(-1)$. Then any continuous $\Gamma_{K}$-morphism $H_{U}^{P} \rightarrow N$ factors through $H_{U}^{T}$.

Proof. This follows immediately from the construction of $H_{U}^{T}$.

Now let us define the subquotient $H_{X}^{T}$ of $H_{U}$ as follows: First, let $H_{U}^{F} \subseteq H_{U}$ denote the inverse image of $H_{X}^{F} \subseteq H_{X}$ under the projection $H_{U} \rightarrow H_{X}$ (cf. the discussion following Definition 6.3 for the definition of $H_{X}^{F}$ ). Now let $H_{X}^{T}$ be the quotient of $H_{U}^{F}$ by the kernel of $H_{U}^{P} \rightarrow H_{U}^{T}$. Thus, we have an exact sequence of topological $\Gamma_{K}$-modules

$$
0 \rightarrow H_{U}^{T} \rightarrow H_{X}^{T} \rightarrow H_{X}^{F} \rightarrow 0
$$

Let us take the continuous dual $\operatorname{Hom}_{\mathbf{Z}_{p}}^{\text {cont }}\left(-, \mathbf{Z}_{p}\right)$ of this exact sequence. This gives us a new exact sequence of topological $\Gamma_{K}$-modules

$$
0 \rightarrow C_{X}^{F} \rightarrow C_{X}^{T} \rightarrow C_{U}^{T} \rightarrow 0
$$

(Here, one may think of the " $H$ 's" as standing for "homology," and the " $C$ 's" as standing for "cohomology.") In particular, we have

$$
C_{U}^{T}=\left(\bigoplus_{\wp \neq x} Z_{\wp}^{T}\right)^{\wedge}
$$

where $Z_{\wp}^{T}$ is defined to be the dual of $I_{\wp}^{T}$. If we pull-back this last exact sequence by $Z_{\wp}^{T} \subseteq C_{U}^{T}$, we obtain exact sequences of topological $\Gamma_{K}$-modules

$$
0 \rightarrow C_{X}^{F} \rightarrow C_{\wp}^{T} \rightarrow Z_{\wp}^{T} \rightarrow 0
$$

all of which are of finite rank over $\mathbf{Z}_{p}$. Moreover, it is clear that that $C_{X}^{T}$ is obtained by summing these extensions (over $\wp$ ) and then $p$-adically completing.

We would like to show that $C_{X}^{T}$ is "Hodge-Tate". Unfortunately, typically the theory of Hodge-Tate Galois representations only goes through for modules of finite rank over $\mathbf{Z}_{p}$. On the other hand, $C_{\wp}^{T}$ is of finite rank over $\mathbf{Z}_{p}$. Thus, our approach in the following will be to use the $C_{\wp}^{T}$ to show that $C_{X}^{T}$ is, in some sense, like a Hodge-Tate representation.

First, let $X_{\wp}$ be the singular curve obtained from $X_{K}$ by considering the subsheaf of $\mathcal{O}_{X_{K}}$ of functions $f$ such that $f(x)=f(\wp)$. Let $J_{\wp}^{\prime}$ be the generalized Jacobian associated to $X_{\wp}$ (see, e.g., [Ser1], Chapitre I, $\S 1$ ). Thus, $J_{\wp}^{\prime}$ is an extension of the abelian variety $J_{X}$ by some torus. Moreover, the $p$-adic Tate module $T\left(J_{\wp}^{\prime}\right)$ of $J_{\wp}^{\prime}$ fits into an exact sequence of $\Gamma_{K}$-modules 


$$
0 \rightarrow I_{\wp} \rightarrow T\left(J_{\wp}^{\prime}\right) \rightarrow H_{X} \rightarrow 0
$$

In particular, one sees immediately that there exists a quotient $J_{\wp}^{\prime} \rightarrow J_{\wp}$ whose $p$-adic Tate module fits into the exact sequence

$$
0 \rightarrow I_{\wp}^{T} \rightarrow T_{p}\left(J_{\wp}\right) \rightarrow H_{X} \rightarrow 0
$$

obtained by pushing forward the preceding exact sequence by $I_{\wp} \rightarrow I_{\wp}^{T}$. Moreover, $C_{\wp}^{T}$ may be identified with the dual of the pull-back of this exact sequence by $H_{X}^{F} \hookrightarrow H_{X}$. Alternatively, $C_{\wp}^{T}$ may be identified with a certain quotient $T_{p}\left(J_{\wp}\right)^{\vee}=H^{1}\left(J_{\wp}, \mathbf{Z}_{p}\right) \rightarrow C_{\wp}^{T}$ (whose kernel is the dual of $H_{X} / H_{X}^{F}$ ). Note, in particular, that $J_{\wp}$ is an extension of $J_{X}$ by the trivial torus $\mathbf{G}_{m}$. Thus, $J_{\wp}$ extends to a semi-abelian variety over $\mathcal{O}_{K}$ (cf. the paragraph following Theorem 2.6 of Chapter I of [FC]).

Next, let us recall (from the theory of $[\mathrm{FC}]$, Chapters I through III) that $J_{X}$ may be constructed as a certain rigid analytic quotient of a semi-abelian variety $\widetilde{J}_{X}$ which has good reduction (i.e., the dimension of the torus part is constant) over $\mathcal{O}_{K}$ by some discrete group. The kernel of the induced pull-back map on cohomology $H^{1}\left(J_{X}, \mathbf{Z}_{p}\right) \rightarrow H^{1}\left(\widetilde{J}_{X}, \mathbf{Z}_{p}\right)$ is precisely the dual of $H_{X} / H_{X}^{F}$. If we pull-back the extension $J_{\wp} \rightarrow J_{X}$ from $J_{X}$ to $\widetilde{J}_{X}$, we then obtain an extension $\widetilde{J}_{\wp}$ of $\widetilde{J}_{X}$ by the trivial torus of dimension one. Moreover, $\widetilde{J}_{\wp}$ has good reduction over $\mathcal{O}_{K}$. Finally, the quotient $H^{1}\left(J_{\wp}, \mathbf{Z}_{p}\right) \rightarrow H^{1}\left(\widetilde{J}_{\wp}, \mathbf{Z}_{p}\right)$ is precisely the quotient $H^{1}\left(J_{\wp}, \mathbf{Z}_{p}\right) \rightarrow C_{\wp}^{T}$ referred to in the preceding paragraph.

Now, just as in Section 9, we can apply the theory of [Falt1] (in the case of good reduction $)$ to $\widetilde{J}_{\wp}$. Let $\mathcal{F}_{0} \stackrel{\text { def }}{=} H^{1}\left(X, \mathcal{O}_{X}\right)$, where $X \rightarrow \operatorname{Spec}\left(\mathcal{O}_{K}\right)$ is the stable extension of $X_{K}$ over $\mathcal{O}_{K}$. Let $\mathcal{F}_{\wp}$ be the space of invariant differentials on $\widetilde{\mathcal{J}}_{\wp}$, where $\widetilde{\mathcal{J}}_{\wp} \rightarrow \operatorname{Spec}\left(\mathcal{O}_{K}\right)$ is the extension of $\widetilde{J}_{\wp}$ to a semi-abelian variety over $\mathcal{O}_{K}$. Thus, $\mathcal{F}_{0}$ and $\mathcal{F}_{\wp}$ are both free of finite rank over $\mathcal{O}_{K}$. Then it follows from the theory of [Falt1] (specifically, Theorems 2.4 and 3.1 of II. of [Falt1]) that

$(*)^{\wp}$ There exists a natural $\Gamma_{K}$-equivariant, $\widehat{\mathcal{O}}_{\bar{K}}$-linear morphism

$$
C_{\wp}^{T} \otimes_{\mathbf{z}_{p}} \widehat{\mathcal{O}}_{\bar{K}} \rightarrow\left(\mathcal{F}_{0} \otimes_{\mathcal{O}_{K}} \widehat{\mathcal{O}}_{\bar{K}}\right) \oplus\left(\mathcal{F}_{\wp} \otimes_{\mathcal{O}_{K}} \widehat{\mathcal{O}}_{\bar{K}}(-1)\right)
$$

which has an inverse up to $p^{n}$, where $n$ is a nonnegative integer that is independent of $\wp$.

Note that the fact that $n$ is independent of $\wp$ is of profound importance in what follows. Moreover, if, instead of $\widetilde{J}_{\wp}$, we had worked with $J_{\wp}$ (which, in general, has "bad (though stable) reduction"), or $J_{\wp}^{\prime}$ (which has only potentially stable reduction - i.e., for general $\wp$, one needs to enlarge $K$ in order to get stable reduction), we would have been unable to obtain this crucial "independence of $\wp$ " from the theory of [Falt1]. 
Now observe that $\mathcal{F}_{\wp}$ fits into a natural exact sequence

$$
0 \rightarrow \mathcal{F}_{X}^{F} \rightarrow \mathcal{F}_{\wp} \rightarrow \mathcal{Z}_{\wp} \rightarrow 0
$$

where $\mathcal{F}_{X}^{F} \stackrel{\text { def }}{=} H^{0}\left(X, \omega_{X / \mathcal{O}_{K}}\right)$, and $\mathcal{Z}_{\wp}$ is defined so as to make the above sequence exact. Now let $\mathcal{F}_{X}^{T}$ be the sum of these extensions over all $\wp$; let $\mathcal{F}_{U}^{T}$ be the direct sum of the $\mathcal{Z}_{\wp}$ over all $\wp$. Thus, we have an exact sequence of flat $\mathcal{O}_{K}$-modules

$$
0 \rightarrow \mathcal{F}_{X}^{F} \rightarrow \mathcal{F}_{X}^{T} \rightarrow \mathcal{F}_{U}^{T} \rightarrow 0
$$

Thus, by taking the $p$-adic completion of the sum of the morphisms in $(*)^{\wp}$, and using the facts that:

(i) the " $n$ " of $(*)^{\wp}$ is independent of $\wp$;

(ii) both $C_{X}^{T}$ and $\mathcal{F}_{X}^{T}$ are sums of extensions which are of finite rank;

we obtain the following result:

Proposition 11.3. We have a natural, $\widehat{\mathcal{O}}_{\bar{K}}$-linear morphism of exact sequences of topological $\Gamma_{K}$-modules

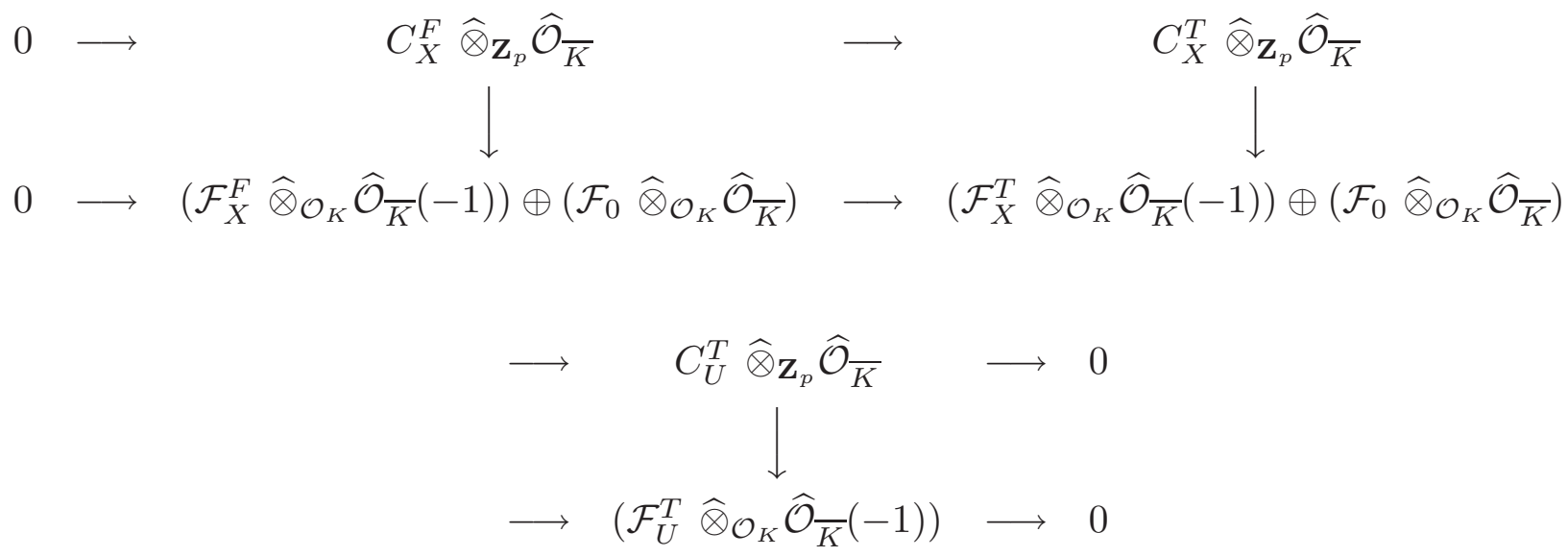

which become isomorphisms when tensored with $\mathbf{Q}_{p}$.

Next, we would like to relate $\mathcal{F}_{X}^{T}$ to a certain space of differentials on $X$, as follows: Let $E$ be a finite set of closed points of $X_{K}$. Let $S \stackrel{\text { def }}{=} \operatorname{Spec}\left(\mathcal{O}_{\bar{K}}\right)$, and let us endow $S$ with the $\log$ structure defined by the monoid $\mathcal{O}_{S}-\{0\}$; denote the resulting log scheme by $S^{\log }$. Let $X^{\log }[E] \rightarrow S^{\log }$ be the pointed stable log-curve (see, e.g., [Mzk3], Section 3, for more details on this terminology) extending $X_{K} \otimes_{K} \bar{K}$, equipped with all the points 
of $X_{K}(\bar{K})$ that map to primes in $E$ as marked points. Let $\mathcal{L}[E] \stackrel{\text { def }}{=} \omega_{X^{\log }[E] / S^{\log }}$. Then if $E \subseteq E^{\prime}$, we get a natural morphism $X^{\log }\left[E^{\prime}\right] \rightarrow X^{\log }[E]$, which lies under a morphism of sheaves $\mathcal{L}[E] \rightarrow \mathcal{L}\left[E^{\prime}\right]$. Thus, by taking the projective limit of the $\left(X^{\log }[E], \mathcal{L}[E]\right)$ as $E$ ranges over all finite sets of closed points of $X_{K}$, we obtain $\left(X^{\log }[\infty], \mathcal{L}[\infty]\right)$. Moreover, note that $H^{0}(X[\infty], \mathcal{L}[\infty])$ is a flat, $p$-adically separated $\mathcal{O}_{\bar{K}}$-module.

If $E$ contains $x$ and $\wp$, then it is easy to see that we get a natural inclusion $\mathcal{F}_{\wp} \hookrightarrow$ $H^{0}(X[E], \mathcal{L}[E])$, which extends to a morphism $\mathcal{F}_{\wp} \otimes_{\mathcal{O}_{K}} \mathcal{O}_{\bar{K}} \hookrightarrow H^{0}(X[E], \mathcal{L}[E])$ whose cokernel is torsion free. Taking the limit with respect to $E$, we thus obtain a map $\mathcal{F}_{\wp} \otimes_{\mathcal{O}_{K}}$ $\mathcal{O}_{\bar{K}} \hookrightarrow H^{0}(X[\infty], \mathcal{L}[\infty])$. In fact, it is not difficult to see that, by summing over the $\mathcal{F}_{\wp}$ for all $\wp \neq x$, we get a morphism

$$
\mathcal{F}_{X}^{T} \otimes \mathcal{O}_{K} \mathcal{O}_{\bar{K}} \hookrightarrow \mathcal{F}_{\infty} \stackrel{\text { def }}{=} H^{0}(X[\infty], \mathcal{L}[\infty])
$$

whose cokernel is torsion free. Moreover, this morphism remains injective after $p$-adic completion. Thus, we see from Proposition 11.3 that we get a morphism

$$
\Phi: C_{X}^{T} \widehat{\otimes}_{\mathbf{z}_{p}} \widehat{\mathcal{O}}_{\bar{K}} \rightarrow \mathcal{F}_{\infty}^{\wedge}(-1)
$$

(where the " $\wedge$ " denotes $p$-adic completion). Moreover, the kernel of $\Phi$ is of weight zero. Finally, if $N$ is a positive integer, let $\mathcal{G}_{\infty}[N] \stackrel{\text { def }}{=} H^{0}\left(X[\infty], \mathcal{L}[\infty]^{\otimes N}\right)$. Thus, $\mathcal{G}_{\infty}[N]$ is a free $\mathcal{O}_{\bar{K}}$-module of infinite rank (such that $\mathcal{G}_{\infty}[1]=\mathcal{F}_{\infty}$ ), and we have a natural multiplication morphism

$$
\otimes_{\mathcal{O}_{\bar{K}}}^{N} \mathcal{F}_{\infty} \rightarrow \mathcal{G}_{\infty}[N]
$$

\section{Section 12: The Preservation of Relations}

The purpose of this Section is to show how the material of Section 11 will be applied in Section 13 to show the "preservation of relations." We maintain the notation of Section 11. Moreover, let us assume that we have been given a continuous open homomorphism over $\Gamma_{K}$

$$
\theta: \Pi_{U_{K}} \rightarrow \Pi_{Y_{K}}
$$

(where $Y_{K} \rightarrow \operatorname{Spec}(K)$ is a proper hyperbolic curve over $K$ ). For simplicity, let us assume (by enlarging $K$ ) that $Y_{K}$ extends to a stable curve $Y \rightarrow \operatorname{Spec}\left(\mathcal{O}_{K}\right)$ over $\mathcal{O}_{K}$, and that the $\Gamma_{K}$-action on $H_{Y}^{M}(-1)$ (cf. the discussion following Definition 6.3 for an explanation of the notation " $H^{M}$ ") is trivial. Next, observe that $\theta$ induces a continuous morphism of $\Gamma_{K}$-modules $H_{U} \rightarrow H_{Y}$ whose cokernel is torsion. Note that this morphism induces 
a map $H_{U}^{F} \rightarrow H_{Y}^{F}$ (cf. the discussions following Definition 6.3 and Lemma 11.2 for an explanation of the notation " $H^{F}$ ") whose cokernel is torsion. Moreover, the restriction of this morphism to $H_{U}^{P}$ (cf. the discussion at the beginning of Section 11 for an explanation of the notation " $H_{U}^{P}$ ") maps into $H_{Y}^{M}$, hence (by Lemma 11.2 and the fact that $\Gamma_{K}$ acts trivially on $H_{Y}^{M}(-1)$ ), we get a morphism $H_{X}^{T} \rightarrow H_{Y}^{F}$ whose cokernel is torsion. Taking the dual of this morphism yields an injection $C_{Y}^{F} \hookrightarrow C_{X}^{T}$ (where $C_{Y}^{F}$ is the dual of $H_{Y}^{F}$ ). Topologically tensoring with $\mathcal{O}_{\bar{K}}$, dividing out by the "weight zero part," and applying the morphism $\Phi$ considered at the end of Section 11, we thus obtain (after further tensoring with $\left.\mathbf{Z}_{p}(1)\right)$ an injection

$$
H^{0}\left(Y, \omega_{Y / \mathcal{O}_{K}}\right) \otimes_{\mathcal{O}_{K}} \widehat{\mathcal{O}}_{\bar{K}} \hookrightarrow \mathcal{F}_{\infty}^{\wedge}
$$

In this Section, we shall be concerned with the issue of whether or not this morphism "preserves relations." By this, we mean the following: Let $N$ be a positive integer. By taking the $N^{\text {th }}$ tensor power of this morphism, and then composing with the completion of the multiplication morphism $\otimes^{N} \mathcal{F}_{\infty} \rightarrow \mathcal{G}_{\infty}[N]$ considered at the end of Section 11, we obtain a morphism

$$
\Psi_{N}: \otimes^{N}\left(H^{0}\left(Y, \omega_{Y / \mathcal{O}_{K}}\right) \otimes_{\mathcal{O}_{K}} \widehat{\mathcal{O}}_{\bar{K}}\right) \rightarrow\left(\mathcal{G}_{\infty}[N]\right)^{\wedge}
$$

We would like to know whether or not $\Psi_{N}$ annihilates the elements of

$$
\mathcal{R}_{N} \stackrel{\text { def }}{=} \operatorname{Ker}\left(\otimes^{N}\left(H^{0}\left(Y, \omega_{Y / \mathcal{O}_{K}}\right)\right) \rightarrow H^{0}\left(Y, \omega_{Y / \mathcal{O}_{K}}^{\otimes N}\right)\right)
$$

i.e., the "relations." In the following, we would like to state a certain assertion that will be proven in the next Section, and explain why this assertion implies that $\Psi_{N}$ "preserves relations."

First, let us introduce the notation necessary to state the assertion. Suppose that $E$ is a finite set of points of $X_{K}(K)$. Then the pointed stable curve $X^{\log }[E] \rightarrow \operatorname{Spec}\left(\mathcal{O}_{\bar{K}}\right)$ introduced at the end of Section 11 is defined over $K$, i.e., we have $X^{\log }[E]_{\mathcal{O}_{K}} \rightarrow \operatorname{Spec}\left(\mathcal{O}_{K}\right)$. Let $\mathfrak{p} \in X[E]_{\mathcal{O}_{K}}$ be a generic point of the special fiber of $X[E]_{\mathcal{O}_{K}}$. Let $\operatorname{Spf}\left(\mathcal{O}_{L}\right)$ be the completion of the localization of $X[E]_{\mathcal{O}_{K}}$ at $\mathfrak{p}$. Thus, $L$ (the quotient field of $\mathcal{O}_{L}$ ) is a $p$-adic field whose residue field is $k(\mathfrak{p})$ (i.e., the residue field of $X[E]_{\mathcal{O}_{K}}$ at the prime $\mathfrak{p}$ ), and we have a canonical $L$-valued point $\xi^{L} \in X_{K}(L)$. Let $\Omega_{L / K}$ denote the module of $p$-adically continuous differentials of $L$ over $K$. Thus, $\Omega_{L / K}$ is an $L$-vector space of dimension one. Moreover, note that we have a natural restriction morphism (induced by $\xi^{L}$ )

$$
\mathcal{F}_{\infty}^{\wedge} \rightarrow \Omega_{L / K} \widehat{\otimes}_{K} \widehat{\bar{K}}
$$

Thus, if we compose this morphism with the morphism

$$
H^{0}\left(Y, \omega_{Y / \mathcal{O}_{K}}\right) \otimes_{\mathcal{O}_{K}} \widehat{\mathcal{O}}_{\bar{K}} \hookrightarrow \mathcal{F}_{\infty}^{\wedge}
$$


considered above, we get a morphism

$$
\kappa_{L}: H^{0}\left(Y, \omega_{Y / \mathcal{O}_{K}}\right) \otimes_{\mathcal{O}_{K}} \widehat{\mathcal{O}}_{\bar{K}} \rightarrow \Omega_{L / K} \widehat{\otimes}_{K} \widehat{\bar{K}}
$$

Next, let us observe that the coverings of $X_{K}$ defined by subgroups of $\Pi_{U_{K}}$ are étale at the point $\xi^{L}$. Thus, it follows that $\xi^{L}$ induces a natural morphism $\alpha_{U}^{L}: \Gamma_{L} \rightarrow \Pi_{U_{K}}$ (well-defined up to conjugation by an element of $\Delta_{U}$ ) whose composite with $\Pi_{U_{K}} \rightarrow \Gamma_{K}$ is the natural morphism $\Gamma_{L} \rightarrow \Gamma_{K}$. Let $\alpha_{Y}^{L}: \Gamma_{L} \rightarrow \Pi_{Y_{K}}$ be the composite of $\alpha_{U}^{L}$ with $\theta$. Then the assertion that will be proven in Section 13 is the following:

$(*)^{L-p t}$ Suppose that $\mathfrak{p}$ satisfies the condition that $\kappa_{L}$ is not identically zero. Then it follows that there exists a point $\left(\xi^{\prime}\right)^{L} \in Y_{K}(L)$ such that $\alpha_{Y}^{L}$ arises from $\left(\xi^{\prime}\right)^{L}$.

Note in particular, that if $\alpha_{Y}^{L}$ arises from $\left(\xi^{\prime}\right)^{L}$, then it follows immediately that the grouptheoretically constructed morphism $\kappa_{L}$ coincides (cf. the application of the theory of [Falt1] discussed in Section 9) with the natural restriction map on differentials induced by $\left(\xi^{\prime}\right)^{L}$. Thus, if $\rho \in \mathcal{R}_{N}$ is a relation, then it follows that the restriction of $\Psi_{N}(\rho) \in\left(\mathcal{G}_{\infty}[N]\right)^{\wedge}$ to $\Omega_{L / K}^{\otimes N} \widehat{\otimes}_{K} \widehat{\bar{K}}$ is zero. This argument already leads one to believe that there should be some sort of connection between $(*)^{L-p t}$ and the "preservation of relations." In fact, we have the following:

Proposition 12.1. $\quad$ Suppose that $(*)^{L-p t}$ always holds (i.e., for all data of the sort discussed above). Then $\Psi_{N}\left(\mathcal{R}_{N}\right)=0$, for all positive integers $N$.

Proof. Suppose that $\rho \in \mathcal{R}_{N}$ is such that $\psi^{\prime} \stackrel{\text { def }}{=} \Psi_{N}(\rho) \neq 0$. By dividing $\psi^{\prime}$ by a suitable element of $\mathcal{O}_{\bar{K}}$, we obtain an element $\psi \in\left(\mathcal{G}_{\infty}[N]\right)^{\wedge}$ such that $\psi \not \equiv 0\left(\bmod \mathfrak{m}_{\bar{K}}\right)$ (where $\mathfrak{m}_{\bar{K}} \subseteq \mathcal{O}_{\bar{K}}$ is the maximal ideal). Let us write $\bar{k}$ for the residue field $\mathcal{O}_{\bar{K}} / \mathfrak{m}_{\bar{K}}$. Write $\bar{\psi}$ for $\psi$ considered modulo $\mathfrak{m}_{\bar{K}}$. Then $\bar{\psi}$ is a section of $\mathcal{L}[E]^{\otimes N} \otimes \bar{k}$ for some $E$. By enlarging $K$, we may assume that $E$ consists solely of $K$-valued points of $X_{K}$. Moreover, since $\bar{\psi}$ is nonzero, there exists some irreducible component of the special fiber of $X[E] \otimes \bar{k}$ over which $\bar{\psi}$ is nonzero. If we choose $\mathfrak{p}$ (in the above discussion) to be such that $\mathfrak{p} \otimes_{\mathcal{O}_{K}} \mathcal{O}_{\bar{K}}$ is this irreducible component, then it follows that the restriction of $\psi \in\left(\mathcal{G}_{\infty}[N]\right)^{\wedge}$ to $\Omega_{L / K}^{\otimes N} \widehat{\otimes}_{K} \widehat{\bar{K}}$ will be nonzero. Now I claim that $\mathfrak{p}$ satisfies the condition that $\kappa_{L}$ is not identically zero: Indeed, if $\kappa_{L}$ were identically zero, then it would follow that $\kappa_{L}^{\otimes N}$ would be identically zero. But the restriction of $\psi$ to $\Omega_{L / K}^{\otimes N} \widehat{\otimes}_{K} \widehat{\bar{K}}$ (which is assumed to be nonzero) is a nonzero $\bar{K}$-multiple of $\kappa_{L}^{\otimes N}(\rho)$ (which would have to be zero). This contradiction proves the claim.

Thus, we are in a position to apply $(*)^{L-p t}$. As discussed in the paragraph preceding this Proposition, it then follows that the restriction of $\psi$ to $\Omega_{L / K}^{\otimes N} \widehat{\otimes}_{K} \widehat{\bar{K}}$ is zero. Thus, we get a contradiction. This completes the proof of the Proposition. 
Remark. In [Mzk2], where one only considers isomorphisms of $\pi_{1}$ 's, as opposed to homomorphisms as we do here, there is no need to place (as we did in $(*)^{L-\mathrm{pt}}$ ) the condition on $\mathfrak{p}$ that $\kappa_{L}$ be not identically zero. Because in the present context it is necessary to include such a condition on $\mathfrak{p}$ in $(*)^{L-p t}$, the author at first did not see how it would be possible to prove the "preservation of relations" in the present context. However, in fact, in order to prove the preservation of relations (Proposition 12.1), it suffices to consider only $\mathfrak{p}$ for which one knows already that $\kappa_{L}$ is not identically zero. This observation arose in discussions between the author and A. Tamagawa.

\section{Section 13: The Preservation of L-Points}

The purpose of this Section is to verify the assertion $(*)^{L-p t}$ discussed in Section 12. The technique is similar to that employed in [Mzk2] (although we do not assume any knowledge of [Mzk2] in the following discussion). We continue with the notation of the preceding Section. In particular, we assume that we have been given a continuous open homomorphism over $\Gamma_{K}$

$$
\theta: \Pi_{U_{K}} \rightarrow \Pi_{Y_{K}}
$$

Moreover, we assume that $X_{K}$ extends to a stable curve $X \rightarrow \operatorname{Spec}\left(\mathcal{O}_{K}\right)$. Let $\mathfrak{p}$ be as in $(*)^{L-\text { pt }}$. Thus, we have a blow-up $\widetilde{X} \rightarrow X$ (that is, $\widetilde{X}$ is what we denoted by $X[E]_{\mathcal{O}_{K}}$ in Section 12), and $\mathfrak{p}$ is a generic point of the special fiber of $\widetilde{X} \rightarrow \operatorname{Spec}\left(\mathcal{O}_{K}\right)$. Choose a smooth, affine, geometrically connected open neighborhood $W$ of $\mathfrak{p}$ in the special fiber of $\widetilde{X}$. Let $T$ be the affine scheme whose coordinate $\operatorname{ring} R_{T}$ is such that $\operatorname{Spf}\left(R_{T}\right)$ (where we equip $R_{T}$ with the $p$-adic topology) is the completion of $\widetilde{X}$ along $W$. Thus, $T \rightarrow \operatorname{Spec}\left(\mathcal{O}_{K}\right)$ is ( $p$-adically) formally smooth, and $T \otimes k=W$. By abuse of notation, we shall write $\mathfrak{p}$ for the prime of $T$ that maps to the original $\mathfrak{p}$ under $T \rightarrow \widetilde{X}$. Let us write $\eta_{T}$ for the generic point of $T$ (regarded as a scheme). Let $L$ be the quotient field of the $\mathfrak{p}$-adic completion of $\left(R_{T}\right)_{\mathfrak{p}}$. Thus, $L$ is a $p$-adic field whose residue field is $k(W)$ (the function field of $W$ ).

Note that we have natural morphisms $\xi_{X}^{T}: T \rightarrow X ; \xi_{X}^{\eta_{T}}: \eta_{T} \rightarrow X_{K} ; \xi_{X}^{L}: \operatorname{Spec}(L) \rightarrow$ $X_{K} ; \xi_{U}^{\eta_{T}}: \eta_{T} \rightarrow U_{K} ; \xi_{U}^{L}: \operatorname{Spec}(L) \rightarrow U_{K}$. Let $\alpha_{U}^{\eta_{T}}: \Gamma_{\eta_{T}} \rightarrow \Pi_{U_{K}}$ be the morphism determined (up to conjugation by an element of $\Delta_{U}$ ) by $\xi_{U}^{\eta_{T}}$. Similarly, we have $\alpha_{U}^{L}$ : $\Gamma_{L} \rightarrow \Pi_{U_{K}}$. Composing $\alpha_{U}^{\eta_{T}}, \alpha_{U}^{L}$ with $\theta$ gives $\alpha_{Y}^{\eta_{T}}: \Gamma_{\eta_{T}} \rightarrow \Pi_{Y_{K}}, \alpha_{Y}^{L}: \Gamma_{L} \rightarrow \Pi_{Y_{K}}$.

Now let $\Delta_{Y^{\prime}} \subseteq \Delta_{Y}$ be a $p$-derivate (see Definition 0.2) of $\Delta_{Y}$. Thus, $\Delta_{Y^{\prime}}$ is an open, characteristic subgroup of $\Delta_{Y}$. Note that $\alpha_{Y}^{\eta_{T}}$ defines a section $\mathfrak{a}_{Y}^{\eta_{T}}: \Gamma_{\eta_{T}} \rightarrow \Pi_{Y_{\eta_{T}}}$ of $\Pi_{Y_{\eta_{T}}} \rightarrow \Gamma_{\eta_{T}}$. Write $\Pi_{Y_{\eta_{T}^{\prime}}}$ for the open subgroup $\operatorname{Im}\left(\mathfrak{a}_{Y}^{\eta_{T}}\right) \cdot \Delta_{Y^{\prime}} \subseteq \Pi_{Y_{\eta_{T}}}$. Thus, $\Pi_{Y_{\eta_{T}^{\prime}}}$ gives rise to a finite étale covering

$$
Y_{\eta_{T}}^{\prime} \rightarrow Y_{\eta_{T}}
$$


Moreover, there exists some finite étale covering $\eta_{T^{\prime}} \rightarrow \eta_{T}$ such that

$$
Y_{\eta_{T}}^{\prime} \times_{\eta_{T}} \eta_{T^{\prime}} \cong Z_{K^{\prime}}^{Y} \times_{K^{\prime}} \eta_{T^{\prime}}
$$

for some curve $Z_{K^{\prime}}^{Y} \rightarrow \operatorname{Spec}\left(K^{\prime}\right)$, where $K^{\prime}$ is a finite extension of $K$ contained in $K\left(\eta_{T^{\prime}}\right)$. Let $T^{\prime}$ be the normalization of $T$ in $\eta_{T^{\prime}}$. Then $T^{\prime} \rightarrow T$ is finite (since $\eta_{T}$ is of characteristic zero) and generically étale.

By means of $\theta$, we can pull-back the above covering to $U_{\eta_{T}}$ : Thus, we obtain an open subgroup $\Pi_{U_{\eta_{T}}^{\prime}} \subseteq \Pi_{U_{\eta_{T}}}$, which corresponds to some finite étale covering $U_{\eta_{T}}^{\prime} \rightarrow U_{\eta_{T}}$ (which is geometrically connected over $\eta_{T}$ since $\Pi_{U_{\eta_{T}}^{\prime}}$ contains the graph of $\alpha_{U}^{\eta_{T}}$ in $\Pi_{U_{\eta_{T}}}=$ $\Gamma_{\eta_{T}} \times_{\Gamma_{K}} \Pi_{U_{K}}$ ). This covering extends to a finite, possibly ramified covering $X_{\eta_{T}}^{\prime} \rightarrow X_{\eta_{T}}$. Moreover, we have an isomorphism

$$
X_{\eta_{T}}^{\prime} \times_{\eta_{T}} \eta_{T^{\prime}} \cong Z_{K^{\prime}}^{X} \times_{K^{\prime}} \eta_{T^{\prime}}
$$

for some curve $Z_{K^{\prime}}^{X} \rightarrow \operatorname{Spec}\left(K^{\prime}\right)$. By enlarging $K^{\prime}$, we may assume that this curve has a stable extension $Z^{X} \rightarrow \operatorname{Spec}\left(\mathcal{O}_{K^{\prime}}\right)$.

Next, let us go back to considering the morphism $\xi_{U}^{\eta_{T}}: \eta_{T} \rightarrow U_{K}$. Now it follows tautologically from the way we defined $\alpha_{Y}^{\eta_{T}}$ (i.e., the fact that it comes originally from $\xi_{U}^{\eta_{T}}$ ) that $\xi_{U}^{\eta_{T}}$ lifts naturally to a point $\xi_{U^{\prime}}^{\eta_{T}}: \eta_{T} \rightarrow U_{\eta_{T}}^{\prime}$. Let $\xi_{X^{\prime}}^{\eta_{T}}: \eta_{T} \rightarrow X_{\eta_{T}}^{\prime}$ be the result of composing this morphism with $U_{\eta_{T}}^{\prime} \rightarrow X_{\eta_{T}}^{\prime}$. Moreover, by restricting to $\eta_{T^{\prime}}$, we get a point $\xi_{X^{\prime}}^{\eta_{T^{\prime}}}: \eta_{T^{\prime}} \rightarrow X_{\eta_{T^{\prime}}}^{\prime}$. Projecting to $Z_{K^{\prime}}^{X}$, we thus get a point $\xi_{Z^{X}}^{\eta_{T^{\prime}}}: \eta_{T^{\prime}} \rightarrow Z_{K^{\prime}}^{X}$. Moreover, it is easy to see that this morphism extends to a morphism $\xi_{Z^{X}}^{T^{\prime \prime}}: T^{\prime \prime} \rightarrow Z^{X}$, where $T^{\prime \prime} \subseteq T^{\prime}$ is an open subscheme obtained as the " $D(f)$ " (i.e., the complement of $V(f) \subseteq T^{\prime}$ ) for some $f \in \Gamma\left(T^{\prime}, \mathcal{O}_{T^{\prime}}\right)$ with the property that $f$ is nonzero at every generic point of the special fiber of $T^{\prime} \rightarrow \operatorname{Spec}\left(\mathcal{O}_{K^{\prime}}\right)$. Indeed, this follows from the following two facts: (i) we already have an extension to $T_{K^{\prime}}^{\prime}$, since $Z_{K^{\prime}}^{X} \rightarrow X_{K}$ is finite, and $\xi_{X}^{\eta_{T}}: \eta_{T} \rightarrow X_{K}$ extends to $T_{K}$; (ii) to extend from $T_{K^{\prime}}^{\prime}$ to some $T^{\prime \prime}$, it suffices to apply the valuative criterion for properness (since $Z^{X} \rightarrow \operatorname{Spec}\left(\mathcal{O}_{K^{\prime}}\right)$ is proper).

Next, let us consider the morphism $T^{\prime \prime} \subseteq T^{\prime} \rightarrow T$. Clearly (after possibly enlarging $\left.K^{\prime}\right)$ there exists a closed point $t \in T$ such that if we let $S$ be the completion of $T$ at $t$, then the morphism $S^{\prime \prime} \rightarrow S$ obtained by base-changing $T^{\prime \prime} \rightarrow T$ by $S \rightarrow T$ has the following properties: (i) $S^{\prime \prime} \rightarrow S$ is finite and flat; (ii) $S^{\prime \prime}$ is a finite disjoint sum of connected components which are geometrically irreducible over $\mathcal{O}_{K^{\prime}}$; (iii) each of these components admits a section over $\mathcal{O}_{K^{\prime}}$. Let $S^{\prime}$ be any one of these connected components. Then we have the following:

Lemma 13.1. The scheme $S^{\prime}$ is normal.

Proof. (Note that this is not entirely obvious since it is not clear that $T$ is excellent.) First, I claim that the (flat) morphism $S \rightarrow T$ is geometrically regular. Indeed, over the generic 
fiber, this follows from the fact that $S$ is regular and generically of characteristic zero; over a closed point of $T_{K}$ or $T$, either the fiber of the morphism $S \rightarrow T$ is (schematically) isomorphic to the given closed point, or it is empty. Thus, it remains to check what happens over the height one prime of $T$ which is the special fiber of $T \rightarrow \operatorname{Spec}\left(\mathcal{O}_{K}\right)$. But over this prime, the geometric regularity of $S \rightarrow T$ follows from the excellence of $T \otimes k=W$ (which is finitely generated over a finite field). This completes the proof of the claim. Thus, it follows that $S^{\prime} \rightarrow T^{\prime}$ is geometrically regular. Moreover, $T^{\prime}$ is normal; hence, we obtain that $S^{\prime}$ is normal, as desired.

Thus, $S^{\prime} \rightarrow S$ satisfies the properties listed in Definition 6.3. Moreover, if we apply the base-change $S \rightarrow T$ to all the objects in the above discussion, it is easy to see that we are in the situation discussed in Proposition 7.4. Note, in particular, that the existence of the morphism $\xi_{Z^{X}}^{T^{\prime \prime}}: T^{\prime \prime} \rightarrow Z^{X}$ shows that the condition $(*)^{S^{\prime}}$ (cf. the discussion preceding Definition 6.4) is satisfied by $\alpha_{X^{\prime}}^{\eta_{S}}: \Gamma_{\eta_{S}} \rightarrow \Pi_{X_{\eta_{S}}^{\prime}}$. In fact, we also have the following:

Lemma 13.2. $\quad \alpha_{X^{\prime}}^{\eta_{S}}$ is F-geometric, hence also FI-geometric.

Proof. Indeed, first observe that restricting $\xi_{Z^{X}}^{T^{\prime \prime}}$ gives rise to a morphism $\xi_{1}: S^{\prime} \rightarrow Z^{X}$. Since $S^{\prime}$ was constructed so as to admit a section over $\mathcal{O}_{K^{\prime}}$, let $\sigma: \operatorname{Spec}\left(\mathcal{O}_{K^{\prime}}\right) \rightarrow S^{\prime}$ be such a section. Then we may form the composite $\xi_{\sigma}: S^{\prime} \rightarrow Z^{X}$ of the structure morphism $S^{\prime} \rightarrow \operatorname{Spec}\left(\mathcal{O}_{K^{\prime}}\right)$ with $\xi_{1} \circ \sigma$. Now observe that $\xi_{1}$ and $\xi_{\sigma}$ both define sections of $Z_{S^{\prime}}^{X} \stackrel{\text { def }}{=} Z^{X} \times \mathcal{O}_{K^{\prime}} S^{\prime} \rightarrow S^{\prime}$ (which coincide over $\operatorname{Im}(\sigma)$ ). Let $D_{1}, D_{\sigma} \subseteq Z_{S^{\prime}}^{X}$ be the Weil divisors which are the images of these two sections. Next, let us observe that by Lemma 13.3 below, these two Weil divisors are Q-Cartier. Hence it follows that there exists a positive integer $N$ such that $N \cdot D_{1}$ and $N \cdot D_{\sigma}$ are, in fact, Cartier divisors, so we may form the line bundle $\mathcal{L} \stackrel{\text { def }}{=} \mathcal{O}_{Z_{S^{\prime}}^{X}}\left(N \cdot D_{1}-N \cdot D_{\sigma}\right)$. Note that the line bundle $\mathcal{L}$ is trivial over $\operatorname{Im}(\sigma) \subseteq S^{\prime}$, hence over the closed point of $S^{\prime}$. Let $\mathcal{J}_{Z^{X}} \rightarrow \operatorname{Spec}\left(\mathcal{O}_{K^{\prime}}\right)$ be the unique semiabelian scheme whose generic fiber is the Jacobian of $Z_{K^{\prime}}^{X}$. Then it follows that $\mathcal{L}$ defines an $S^{\prime}$-valued point of $\mathcal{J}_{Z^{x}}$ which arises from an $S^{\prime}$-valued point of the formal completion of $\mathcal{J}_{Z^{X}}$ at the identity. But, sorting through the definitions (in particular, Definitions 6.2, 6.4) reveals that this implies that $\alpha_{X^{\prime}}^{\eta_{S}}$ is F-geometric, hence also FI-geometric (cf. the paragraph following Defintion 6.4).

Lemma 13.3. The scheme $Z_{S^{\prime}}^{X} \stackrel{\text { def }}{=} Z^{X} \times_{\mathcal{O}_{K^{\prime}}} S^{\prime}$ is normal, and, moreover, any Weil divisor $D \subseteq Z_{S^{\prime}}^{X}$ arising as the graph of an $\mathcal{O}_{K^{\prime}-m o r p h i s m} \xi_{D}: S^{\prime} \rightarrow Z^{X}$ is $\mathbf{Q}$-Cartier.

Proof. By Lemma 13.1 above, $S^{\prime}$ is normal. Moreover, since $Z_{S^{\prime}}^{X}$ is a generically smooth stable curve over $S^{\prime}$, one checks easily that the conditions " $R_{1}$ " and " $S_{2}$ " of Serre (see, e.g., [Mats], Chapter 7, §17.I, Theorem 39) are satisfied by $Z_{S^{\prime}}^{X}$, so $Z_{S^{\prime}}^{X}$ is normal. Now recall that to say that a Weil divisor is "Q-Cartier" simply means that some nonzero multiple of that Weil divisor is Cartier. Next, let us observe that since the graph of $\xi_{D}$ is defined by a 
single equation in a neighborhood of any point of the smooth locus of $Z^{X} \rightarrow \operatorname{Spec}\left(\mathcal{O}_{K^{\prime}}\right)$, it suffices to check that $D$ is Q-Cartier in a neighborhood of the nodes of the special fiber of $Z^{X} \rightarrow \operatorname{Spec}\left(\mathcal{O}_{K^{\prime}}\right)$. In fact, it even suffices to check that $D$ is $\mathbf{Q}$-Cartier over the spectrum of the completion of the local ring of $Z_{S^{\prime}}^{X}$ at such a node.

Thus, let us write

$$
\mathcal{A} \stackrel{\text { def }}{=} \Gamma\left(S^{\prime}, \mathcal{O}_{S^{\prime}}\right) ; \quad \mathcal{B} \stackrel{\text { def }}{=} \mathcal{O}_{K^{\prime}}[[x, y]] /\left(x y-\pi^{n}\right) ; \quad \mathcal{C} \stackrel{\text { def }}{=} \mathcal{A}[[x, y]] /\left(x y-\pi^{n}\right)
$$

where $\pi \in \mathcal{O}_{K^{\prime}}$ is a uniformizer, and $n$ is a positive integer. Here, we think of $\mathcal{B}$ (respectively, $\mathcal{C}$ ) as the result of completing $Z^{X}$ (respectively, $Z_{S^{\prime}}^{X}$ ) at a node (respectively, at the inverse image of this node via the projection $Z_{S^{\prime}}^{X} \rightarrow Z^{X}$ ). Recall that $S^{\prime}$ is affine, so $S^{\prime}=\operatorname{Spec}(\mathcal{A})$. Now $\xi_{D}$ is given by some morphism $\mathcal{B} \rightarrow \mathcal{A}$. Write $s_{x}, s_{y} \in \mathcal{A}$ for the images of $x$ and $y$, respectively, under this morphism. Note that $s_{x} \cdot s_{y}=\pi^{n} \in \mathcal{A}$. Moreover, the restriction $D_{\mathcal{C}}$ of the subscheme $D$ to $\operatorname{Spec}(\mathcal{C})$ is defined by the equations $x-s_{x}, y-s_{y}$, i.e., $D_{\mathcal{C}}=V\left(x-s_{x}, y-s_{y}\right)$. Now let us consider the regular function $x-s_{x}$ on $\operatorname{Spec}(\mathcal{C})$. I claim that the ideals $\left(x-s_{x}\right)$ and $\left(x-s_{x}, y-s_{y}\right)$ in $\mathcal{C}$ coincide in $\mathcal{C}\left[\frac{1}{\pi}\right]$. Indeed, this follows from the following equation:

$$
y-s_{y}=\frac{-y \cdot s_{y}}{\pi^{n}}\left(x-s_{x}\right) \in \mathcal{C}\left[\frac{1}{\pi}\right]
$$

Thus, we conclude that on $\operatorname{Spec}(\mathcal{C})$, the Weil divisor $D_{\mathcal{C}}$ is linearly equivalent to a Weil divisor $E$ on $\operatorname{Spec}(\mathcal{C})$ which is supported on the special fiber $F_{\mathcal{C}}$ of $\operatorname{Spec}(\mathcal{C}) \rightarrow \operatorname{Spec}\left(\mathcal{O}_{K^{\prime}}\right)$. On the other hand, the fact that $S^{\prime} \rightarrow \operatorname{Spec}\left(\mathcal{O}_{K^{\prime}}\right)$ is geometrically irreducible implies that $\left(F_{\mathcal{C}}\right)_{\text {red }}$ has precisely two irreducible components, namely, $V(x)_{\text {red }}$ and $V(y)_{\text {red }}$. Thus, it follows that any divisor supported on $F_{\mathcal{C}}$ is Q-Cartier, as desired. This completes the proof of the Lemma.

Thus, (by Lemma 13.2) we may apply Proposition 7.4 to conclude that $Y_{\eta_{S}}^{\prime}$ admits a line bundle of degree prime to p. Moreover, by Proposition 8.1 and Lemma 8.2, it thus follows that:

$$
\begin{aligned}
& Y_{L}^{\prime} \stackrel{\text { def }}{=} Y_{\eta_{T}}^{\prime} \times{ }_{\eta_{T}} \operatorname{Spec}(L) \text { has a rational point over some tamely ramified } \\
& \text { extension } L^{\prime} \text { of } L \text {. }
\end{aligned}
$$

It is this key result that will allow us to conclude the proof of $(*)^{L-p t}$.

Let us review what we have done so far, from the point of view of objects over $Y_{L}$. First, we have a section $\mathfrak{a}_{Y}^{L}: \Gamma_{L} \rightarrow \Pi_{Y_{L}}$ of $\Pi_{Y_{L}} \rightarrow \Gamma_{L}$ (defined by $\alpha_{Y}^{L}$ ). Moreover, given any $p$-derivate $\Delta_{Y_{L}^{\prime}} \subseteq \Delta_{Y}$, we obtain a finite étale covering $Y_{L}^{\prime} \rightarrow Y_{L}$ defined by $\Pi_{Y_{L}^{\prime}} \stackrel{\text { def }}{=}$ $\operatorname{Im}\left(\mathfrak{a}_{Y}^{L}\right) \cdot \Delta_{Y_{L}^{\prime}} \subseteq \Pi_{Y_{L}}$. Under these circumstances, we just showed that $Y_{L}^{\prime}$ necessarily has 
a rational point over some tamely ramified extension of $L$. Moreover, tracing through the definitions, it is easy to see that the assumption placed on $\mathfrak{p}$ in $(*)^{L-p t}$ that $\kappa_{L}$ be not identically zero means precisely (in the language introduced at the beginning of Section 9) that $\mathfrak{a}_{Y}^{L}$ is nondegenerate. It thus follows that we can apply Corollary 10.5 to conclude that:

$$
\mathfrak{a}_{Y}^{L}: \Gamma_{L} \rightarrow \Pi_{Y_{L}} \text { arises from some geometric point }\left(\xi^{\prime}\right)^{L} \in Y_{L}(L)
$$

In other words, the proof of $(*)^{L-p t}$ has been completed. Thus, by Proposition 12.1 , we conclude the following:

Corollary 13.4. Let $\theta: \Pi_{U_{K}} \rightarrow \Pi_{Y_{K}}$ be a continuous open homomorphism over $\Gamma_{K}$. Then $\theta$ "preserves relations," i.e., (in the notation of Section 12), we have $\Psi_{N}\left(\mathcal{R}_{N}\right)=0$, for all positive integers $N$.

Note that in [Mzk2] (where we essentially dealt with the case where $\theta$ is an isomorphism), the preservation of relations is already enough to conclude the proof of the main theorem. In the present context, however, because of the fact that $H_{U}$ is of infinite rank, it is necessary to go through one more intermediate technical step before we can complete the proof of the (first part of the) first main theorem. This step essentially amounts to showing that any $\theta$ as in Corollary 13.4 necessarily factors through $\Pi_{X_{K}}$. The proof of this next step is the main topic of the following Section. Note that if this fact (i.e., that $\theta$ factors through $\Pi_{X_{K}}$ ) could be proven more directly, then this paper could be simplified considerably. (For instance, Section 11, as well as the rather technical notions of "irreducibly splittable" and "FI-geometric" would be unnecessary.) Unfortunately, however, the proof of this fact in Section 14 relies heavily on the "preservation of relations" (Corollary 13.4).

\section{Section 14: The Annihilation of Inertia}

In this Section, we prove that any continuous surjective homomorphism $\theta: \Pi_{U_{K}} \rightarrow$ $\Pi_{Y_{K}}$ over $\Gamma_{K}$ necessarily (acts as though it) factors through $\Pi_{X_{K}}$. In the process of doing this, we complete the proof of the (first part of) the first main theorem of this paper, in the case where the base field is a local field. Throughout most of this Section (except for the very end - i.e., from the statement of Theorem 14.1 on), we continue to use the notation of the preceding three Sections. Let us assume, moreover, that $Y_{K}$ is not hyperelliptic. Thus, it follows (e.g., from Lemma $10.4(3)$ ) that any connected finite étale covering of $Y_{K}$ is also non-hyperelliptic.

Let us first consider the morphism

$$
H^{0}\left(Y, \omega_{Y / \mathcal{O}_{K}}\right) \otimes_{\mathcal{O}_{K}} \widehat{\mathcal{O}}_{\bar{K}} \hookrightarrow \mathcal{F}_{\infty}^{\wedge}
$$


constructed in Section 12. We would like to show first of all that

$$
\begin{aligned}
& (*)^{\mathcal{F}_{X}^{F}} \text { This morphism factors through } \mathcal{F}_{X}^{F} \otimes_{\mathcal{O}_{K}} \widehat{\mathcal{O}}_{\bar{K}} \subseteq \mathcal{F}_{\infty}^{\wedge} \text {, where } \mathcal{F}_{X}^{F} \stackrel{\text { def }}{=} \\
& H^{0}\left(X, \omega_{X / \mathcal{O}_{K}}\right) \text { (cf. the discussion preceding Proposition 11.3). }
\end{aligned}
$$

To do this, we argue as follows: Let $x \in X_{K}(K)$. Let

$$
\epsilon_{x}: \mathcal{F}_{\infty}^{\wedge} \rightarrow \widehat{\mathcal{O}}_{\bar{K}}
$$

be the morphism induced by restriction: i.e., elements of $\mathcal{F}_{\infty}$ are sections of $\mathcal{L}[\infty]$, hence differentials on $X[\infty]$; thus, by taking the residue of such a differential at $x$, we get a residue map $\mathcal{F}_{\infty} \rightarrow \mathcal{O}_{\bar{K}}$; then taking the $p$-adic completion of this residue map gives us $\epsilon_{x}$. We would like to show in the following that the restriction

$$
\zeta_{x}: H^{0}\left(Y, \omega_{Y / \mathcal{O}_{K}}\right) \rightarrow \widehat{\mathcal{O}}_{\bar{K}}
$$

of $\epsilon_{x}$ to $H^{0}\left(Y, \omega_{Y / \mathcal{O}_{K}}\right)$ is zero. If we show this, then this will be also hold for all $x^{\prime} \in$ $X_{K}\left(K^{\prime}\right)$ (where $K^{\prime}$ is a finite extension of $K$ ), hence $(*)^{\mathcal{F}_{X}^{F}}$ will follow immediately (from the fact that $\mathcal{F}_{X}^{F}$ consists precisely of all those differentials whose residues at every point are zero).

Thus, let us assume that $\zeta_{x} \neq 0$. Let $I_{x} \subseteq \Delta_{U}$ denote the inertia group (well-defined up to conjugation) corresponding to $x$. Then observe that the restriction $I_{x} \subseteq \Delta_{U} \rightarrow \Delta_{Y}$ of $\theta$ to $\Delta_{U}$ is nontrivial. Indeed, if this restriction were zero, then it is clear from the way that $\zeta_{x}$ was constructed (cf. the "comparison theorem" of Proposition 11.3) that $\zeta_{x}$ would be zero. Thus, $\theta\left(I_{x}\right) \neq\{1\}$. In particular, it follows (by possibly enlarging $K$ ) that there exists an open normal subgroup $\Pi_{Y_{K}^{\prime}} \subseteq \Pi_{Y_{K}}$ (corresponding to a covering $Y_{K}^{\prime} \rightarrow Y_{K}$ ) such that: (i) $\Pi_{Y_{K}^{\prime}}$ surjects onto $\Gamma_{K}$; and (ii) if we let $U_{K}^{\prime} \rightarrow U_{K}$ be the result of pulling back $Y_{K}^{\prime} \rightarrow Y_{K}$ via $\theta$, then $U_{K}^{\prime} \rightarrow U_{K}$ is a connected Galois covering which is ramified over $x$. Let $G \stackrel{\text { def }}{=} \Pi_{Y_{K}} / \Pi_{Y_{K}^{\prime}}$. Thus, $G$ is a finite group, and $G=\operatorname{Gal}\left(U_{K}^{\prime} / U_{K}\right)=\operatorname{Gal}\left(Y_{K}^{\prime} / Y_{K}\right)$. Note that $U_{K}^{\prime} \rightarrow U_{K}$ extends to a ramified covering $X_{K}^{\prime} \rightarrow X_{K}$. Let $x^{\prime} \in X_{K}(K)$ (where we enlarge $K$ if necessary) be a point lying above $x$.

Now let us denote with primes the objects corresponding to $U_{K}^{\prime}, X_{K}^{\prime}$, and $Y_{K}^{\prime}$ that are analogous to the objects already constructed for $U_{K}, X_{K}$, and $Y_{K}$. Thus, we have

$$
\epsilon_{x^{\prime}}^{\prime}:\left(\mathcal{F}_{\infty}^{\prime}\right)^{\wedge} \rightarrow \widehat{\mathcal{O}}_{\bar{K}}
$$

and

$$
\zeta_{x^{\prime}}^{\prime}: H^{0}\left(Y^{\prime}, \omega_{Y^{\prime} / \mathcal{O}_{K}}\right) \rightarrow \widehat{\mathcal{O}}_{\bar{K}}
$$


Note that the fact that $\zeta_{x} \neq 0$ implies $\left(\right.$ since $\left.\zeta_{x^{\prime}}^{\prime}\right|_{H^{0}\left(Y, \omega_{Y / \mathcal{O}_{K}}\right)}=\zeta_{x}$ ) that $\zeta_{x^{\prime}}^{\prime} \neq 0$. Let $\sigma \in G$ be such that $\sigma\left(x^{\prime}\right)=x^{\prime}, \sigma \neq 1$. Then the fact that $\sigma$ fixes $x^{\prime}$ implies that $\sigma$ fixes $\epsilon_{x^{\prime}}^{\prime}$, which, in turn, implies (since the inclusion $H^{0}\left(Y^{\prime}, \omega_{Y^{\prime} / \mathcal{O}_{K}}\right) \hookrightarrow\left(\mathcal{F}_{\infty}^{\prime}\right)^{\wedge}$ is G-equivariant) that $\sigma$ fixes $\zeta_{x^{\prime}}^{\prime}$.

On the other hand, by Corollary 13.4 ("preservation of relations") and the fact that $Y_{K}^{\prime}$ is not hyperelliptic, it follows that the point of the projective space $P_{K}^{\prime} \stackrel{\text { def }}{=}$ $\mathbf{P}\left(H^{0}\left(Y^{\prime}, \omega_{Y^{\prime} / \mathcal{O}_{K}}\right)\right)$ defined by $\zeta_{x^{\prime}}^{\prime}$ lies on the canonically embedded curve $Y_{K}^{\prime} \subseteq P_{K}^{\prime}$. Thus, the fact that $\zeta_{x^{\prime}}^{\prime}$ is fixed by $\sigma$ implies that $Y_{K}^{\prime}$ admits a $\widehat{\bar{K}}$-valued point which is fixed by $\sigma$. Since $Y_{K}^{\prime} \rightarrow Y_{K}=Y_{K}^{\prime} / G$ is étale, however, this is absurd. This contradiction thus completes the proof that $\zeta_{x}=0$, and hence also the proof of $(*)^{\mathcal{F}_{X}^{F}}$.

Let us review what we have done so far. Given a surjective continuous homomorphism $\theta: \Pi_{U_{K}} \rightarrow \Pi_{Y_{K}}$ over $\Gamma_{K}$, we have seen that $\theta$ induces an injection

$$
H^{0}\left(Y_{K}, \omega_{Y_{K} / K}\right) \hookrightarrow H^{0}\left(X_{K}, \omega_{X_{K} / K}\right)
$$

that preserves relations. Thus, it follows (by using the canonical embedding of $Y_{K}$ ) that we get a dominant morphism $\theta_{U}: U_{K} \rightarrow Y_{K}$ which extends to a morphism

$$
\theta_{X}: X_{K} \rightarrow Y_{K}
$$

by properness. Moreover, given any finite Galois étale covering $Y_{K}^{\prime} \rightarrow Y_{K}$ whose geometric part has $p$-power order, we can pull-back this covering via $\theta$ to obtain $U_{K}^{\prime} \rightarrow U_{K}$ (finite étale), $X_{K}^{\prime} \rightarrow X_{K}$ (finite and possibly ramified), together with $\theta^{\prime}: \Pi_{U_{K}^{\prime}} \rightarrow \Pi_{Y_{K}^{\prime}}$. If we then repeat the argument just applied to $\theta$ for $\theta^{\prime}$, we see that we get a morphism $\theta_{X^{\prime}}^{\prime}: X_{K}^{\prime} \rightarrow Y_{K}^{\prime}$ which lies over $\theta_{X}$. If we then continue this procedure for arbitrary finite Galois étale coverings (whose geometric parts have $p$-power order), the well-known correspondence between fundamental groups and categories of étale coverings thus shows that the morphism induced by $\theta_{U}$ on fundamental groups coincides with $\theta$ up to composition with an inner automorphism induced by an element of $\Pi_{Y_{K}}$. On the other hand, since both $\theta$ and the morphism induced by $\theta_{U}$ on fundamental groups have the property that they lie over $\Gamma_{K}$, it follows that the element of $\Pi_{Y_{K}}$ in question must map to the the center of $\Gamma_{K}$. Since, however, $\Gamma_{K}$ is center-free (see Lemma 15.6 below - one checks easily that there are no "vicious circles" in the reasoning), it follows that the element in question must therefore be an element of $\Delta_{Y} \subseteq \Pi_{Y_{K}}$. That is to say, we have essentially proven the following result:

Theorem 14.1. $\quad$ Let $K$ be a finite extension of $\mathbf{Q}_{p}$. Let $Y_{K}$ be a hyperbolic curve (not necessarily proper) over $K$. Let $U_{K}$ be the spectrum of a one-dimensional function field over $K$. Let $\operatorname{Hom}_{K}^{\text {dom }}\left(U_{K}, Y_{K}\right)$ be the set of dominant $K$-morphisms from $U_{K}$ to $Y_{K}$. Let $\operatorname{Hom}_{\Gamma_{K}}^{\text {open }}\left(\Pi_{U_{K}}, \Pi_{Y_{K}}\right)$ be the set of open, continuous group homomorphisms $\Pi_{U_{K}} \rightarrow \Pi_{Y_{K}}$ over $\Gamma_{K}$, considered up to composition with an inner automorphism arising from $\Delta_{Y}$. Then the natural map 


$$
\operatorname{Hom}_{K}^{\text {dom }}\left(U_{K}, Y_{K}\right) \rightarrow \operatorname{Hom}_{\Gamma_{K}}^{\text {open }}\left(\Pi_{U_{K}}, \Pi_{Y_{K}}\right)
$$

is bijective.

Proof. We begin by proving that this map is injective. First, observe that by replacing $U_{K}$ and $Y_{K}$ by coverings defined by " $p$-derivates" (as in Definition 0.2) of $\Delta_{U}$ and $\Delta_{Y}$ (where we use $p$-derivates since they are natural), we may assume that both $U_{K}$ and $Y_{K}$ admit hyperbolic compactifications $\bar{U}_{K}$ and $\bar{Y}_{K}$ (i.e., $\bar{U}_{K}$ and $\bar{Y}_{K}$ are proper hyperbolic curves over $K)$. Since dominant $K$-morphisms $U_{K} \rightarrow Y_{K}$ are the same as dominant $K$-morphisms $\bar{U}_{K} \rightarrow \bar{Y}_{K}$, it suffices to show that such a morphism $\bar{U}_{K} \rightarrow \bar{Y}_{K}$ is determined by its induced morphism $\Delta_{\bar{U}}^{\mathrm{ab}} \rightarrow \Delta_{\bar{Y}}^{\mathrm{ab}}$ (on abelianizations of geometric fundamental groups). But this follows from the fact that this morphism $\Delta \frac{\mathrm{ab}}{U} \rightarrow \Delta \frac{\mathrm{ab}}{Y}$ clearly determines the morphism between all $p$-power torsion points of the Jacobians of $\bar{U}_{K}$ and $\bar{Y}_{K}$, hence it determines the induced morphism between the Jacobians of $\bar{U}_{K}$ and $\bar{Y}_{K}$. Moreover, since $\bar{U}_{K}$ and $\bar{Y}_{K}$ are both proper hyperbolic (i.e., of genus $\geq 2$ ), they both embed in their Jacobians, so we conclude that the original morphism $\bar{U}_{K} \rightarrow \bar{Y}_{K}$ is uniquely determined, as desired.

Next, we consider surjectivity. Let us first consider the case where $Y_{K}$ is proper. By replacing $U_{K}$ by a finite étale covering of $U_{K}$ (and then descending at the end, which is possible since (by the preceding paragraph) the natural map in the Theorem is injective), we can assume that the proper model $X_{K}$ of $U_{K}$ is hyperbolic. Note, moreover, that if $\theta: \Pi_{U_{K}} \rightarrow \Pi_{Y_{K}}$ is open, then its image is of finite index, so by replacing $Y_{K}$ by a finite étale covering of $Y_{K}$, it is clear that we may assume that $\theta$ is surjective. Finally, by Lemma 10.4 (4), by replacing $Y_{K}$ by a finite étale covering of $Y_{K}$, it is clear that we may assume that $Y_{K}$ is non-hyperelliptic. Now we are in the circumstances considered above, and so we see that $\theta$ arises from a geometric morphism $U_{K} \rightarrow Y_{K}$, as desired. This completes the proof of the Theorem when $Y_{K}$ is proper.

Now let us consider the case when $Y_{K}$ is not proper. First note that by replacing $Y_{K}$ by a finite étale covering of $Y_{K}$, we may assume that the compactification $Z_{K}$ of $Y_{K}$ is hyperbolic. Now the point is to compose the given $\theta: \Pi_{U_{K}} \rightarrow \Pi_{Y_{K}}$ with the morphism $\Pi_{Y_{K}} \rightarrow \Pi_{Z_{K}}$ arising from the compactification inclusion $Y_{K} \subseteq Z_{K}$. Since we know the Theorem to be true for morphisms between $U_{K}$ and $Z_{K}$, we thus obtain that $\Pi_{U_{K}} \rightarrow \Pi_{Z_{K}}$ arises from some dominant $U_{K} \rightarrow Z_{K}$ (which necessarily factors - since $U_{K}$ is the spectrum of a field - through $Y_{K}$, thus yielding a $U_{K} \rightarrow Y_{K}$ ). Thus it remains only to see that the morphism induced on $\pi_{1}$ 's by this $U_{K} \rightarrow Y_{K}$ is the same as the given $\theta$. But this is done (as usual) by considering a finite étale covering $Y_{K}^{\prime} \rightarrow Y_{K}$, and applying the argument just described to $U_{K}^{\prime}$ and $Y_{K}^{\prime}$ to obtain a $U_{K}^{\prime} \rightarrow Y_{K}^{\prime}$ which lies over the $U_{K} \rightarrow Y_{K}$ constructed previously. As usual, this is enough to show (cf. the argument directly preceding the statement of Theorem 14.1) that the morphism induced on fundamental groups by the $U_{K} \rightarrow Y_{K}$ that we constructed is the same as the given $\theta$. This completes the proof of the Theorem.

In fact, we have the following: 
Corollary 14.2. Let $K$ be a finite extension of $\mathbf{Q}_{p}$. Let $X_{K}$ (respectively, $Y_{K}$ ) be either a hyperbolic curve (not necessarily proper!) over $K$ or the spectrum of a one-dimensional function field over $K$. Let $\operatorname{Hom}_{K}^{\operatorname{dom}}\left(X_{K}, Y_{K}\right)$ be the set of dominant $K$-morphisms from $X_{K}$ to $Y_{K}$. Let $\operatorname{Hom}_{\Gamma_{K}}^{\text {open }}\left(\Pi_{X_{K}}, \Pi_{Y_{K}}\right)$ be the set of open, continuous group homomorphisms $\Pi_{X_{K}} \rightarrow \Pi_{Y_{K}}$ over $\Gamma_{K}$, considered up to composition with an inner automorphism arising from $\Delta_{Y}$. Then the natural map

$$
\operatorname{Hom}_{K}^{\text {dom }}\left(X_{K}, Y_{K}\right) \rightarrow \operatorname{Hom}_{\Gamma_{K}}^{\text {open }}\left(\Pi_{X_{K}}, \Pi_{Y_{K}}\right)
$$

is bijective.

Proof. By an argument analogous to that of the final paragraph of the proof of Theorem 14.1, it follows that it suffices to consider the case where $Y_{K}$ is a hyperbolic curve. If $U_{K}$ is the generic point (considered as a scheme) of $X_{K}$, then given any open $\theta_{X}: \Pi_{X_{K}} \rightarrow \Pi_{Y_{K}}$, composing $\theta_{X}$ with the natural surjection $\Pi_{U_{K}} \rightarrow \Pi_{X_{K}}$ induced by $U_{K} \rightarrow X_{K}$ gives us an open $\theta_{U}: \Pi_{U_{K}} \rightarrow \Pi_{Y_{K}}$. Applying Theorem 14.1 to $\theta_{U}$ thus gives us a dominant morphism $X_{K} \rightarrow \bar{Y}_{K}$ (where $\bar{Y}_{K}$ is the compactification of the curve $Y_{K}$ ). To see that this morphism factors through $Y_{K}$, it suffices to apply this construction to $Y_{K}^{\prime} \rightarrow Y_{K}$, where $Y_{K}^{\prime}$ is a finite, geometrically connected (over $K$ ), Galois étale covering (of $p$-power order) of $Y_{K}$ which is ramified over all the points of $\bar{Y}_{K}-Y_{K}$. In fact, we may even choose $Y_{K}^{\prime}$ so that the ramification indices over all the points of $\bar{Y}_{K}-Y_{K}$ are larger than the degree of $X_{K}$ over $\bar{Y}_{K}$. Then the fact that we get some $X_{K}^{\prime} \rightarrow \bar{Y}_{K}^{\prime}$ (where $X_{K}^{\prime}$ is étale over $X_{K}$ ) lying over the morphism $X_{K} \rightarrow \bar{Y}_{K}$ obtained previously shows that this morphism $X_{K} \rightarrow \bar{Y}_{K}$ factors through $Y_{K}$, as desired. This completes the proof of the Corollary.

Remark. Corollary 14.2 is thus a special case of Theorem $A$, the first main theorem of this paper. In fact, Corollary 14.2 holds even in the case where $K$ is only finitely generated over $\mathbf{Q}_{p}$. However, unlike the situation in [Mzk2], deriving this finitely generated case from the local field case is not so trivial, again (cf. the discussion at the end of Section 13) because of the fact that $\Delta_{U}$ is so large. Thus, we save the derivation of the finitely generated case for the following Section.

\section{Section 15: Base Fields Finitely Generated over the p-adics}

In this Section, we let $L$ be a finitely generated extension of $\mathbf{Q}_{p}$. We would like to prove versions of Theorem 14.1 and Corollary 14.2 in the case where the base field is $L$ (as opposed to a finite extension of $\mathbf{Q}_{p}$ ). Thus, by induction on the transcendence degree of $L$ over $\mathbf{Q}_{p}$, we may assume that Theorem 14.1, for instance, is known for all base fields whose transcendence degree over $\mathbf{Q}_{p}$ is $<$ that of $L$.

Let $X_{L}$ and $Y_{L}$ be proper hyperbolic curves over $L$. Let $U_{L}$ be the generic point of $X_{L}$ (regarded as a scheme). Moreover, let us assume that we have been given a continuous surjective homomorphism over $\Gamma_{L}$ 


$$
\theta: \Pi_{U_{L}} \rightarrow \Pi_{Y_{L}}
$$

Once it is shown that $\theta$ factors though $\Pi_{X_{L}}$, it is relatively easy to derive that $\theta$ arises geometrically from Theorem 14.1. Thus, the first order of business is to show that $\theta$ factors through $\Pi_{X_{L}}$. To achieve this, we assume that this is not the case. Then (cf. the argument employed in Section 14) by replacing $Y_{L}$ and $U_{L}$ by finite étale coverings $Y_{L}^{\prime} \rightarrow Y_{L}$ and $U_{L}^{\prime} \rightarrow U_{L}$ (the latter obtained by pulling back the former via $\theta$ ), we may assume that we are in the following situation: There is a finite cyclic group $G$ (with generator $\sigma$ ) acting faithfully on $U_{L}$ (hence also $X_{L}$ ) and $Y_{L}$ - via L-linear automorphisms - in such a way that $\sigma$ fixes a point $x_{0} \in X_{L}(L)$, but acts without fixed points on $Y_{L}$. Moreover, we assume that $\sigma \circ \theta \circ \sigma^{-1}$ coincides with $\theta$ up to composition with an inner automorphism defined by an element of $\Delta_{Y}$. If we can show that these assumptions lead to a contradiction, it will follow immediately that $\theta$ factors through $\Pi_{X_{L}}$.

Let (as usual) $H_{U}$ (respectively, $H_{Y}$ ) be the abelianization of $\Delta_{U}$ (respectively, $\Delta_{Y}$ ) regarded as a $\Gamma_{K}$-module. Let $H_{U}^{P} \subseteq H_{U}$ be the closure of the image of all the inertia groups (cf. the beginning of Section 11). Note that $\theta$ induces a $\Gamma_{K}$-morphism $H_{U} \rightarrow H_{Y}$. Since $H_{Y}$ is a finitely generated $\mathbf{Z}_{p}$-module, it follows that (by possibly replacing $L$ by a finite extension of $L$ ) there exist points $x_{1}, \ldots, x_{r} \in X_{L}(L)$ such that the images of the corresponding inertia groups $\theta\left(I_{i}\right)$ (for $\left.i=1, \ldots, r\right)$ in $H_{Y}$ is equal to the image of $\theta\left(H_{U}^{P}\right)$ in $H_{Y}$.

Now let $K \subseteq L$ be a subfield such that $L$ is a one-dimensional function field over $K$ (hence, in particular, we assume that $K$ is algebraically closed in $L$ ). Note that such a $K$ always exists (as long as $L$ is not a finite extension of $\mathbf{Q}_{p}$ ). Thus, there exists a smooth affine model $M \rightarrow \operatorname{Spec}(K)$ of $L$ such that $X_{L}$ and $Y_{L}$ extend to smooth curves $X_{M} \rightarrow M$ and $Y_{M} \rightarrow M$ over $M$. Moreover, we may also assume (by shrinking $M$ ) that $x_{1}, \ldots, x_{r}$ extend to sections $s_{1}, \ldots, s_{r}: X_{M} \rightarrow M$ whose images are disjoint from one another, and that $\sigma$ acts on $X_{M}$ and $Y_{M}$. The point $x_{0} \in X_{L}(L)$ then extends (by the valuative criterion for properness) to a section $s_{0}: M \rightarrow X_{M}$. Finally, let us observe that (by further shrinking $M$ ), we may assume that $\sigma$ acts without fixed points on $Y_{M}$.

The first thing that we would like to get our hands on is a morphism like $\theta$, except for objects over $K$, so that we can apply Theorem 14.1 over $K$ (which we know to be true via the induction hypothesis). To construct such a morphism, we argue as follows. Let $m \in M(K)$ be a point (which, after possibly enlarging $K$, always exists). Let $D$ be the spectrum of the completion of $\mathcal{O}_{M, m}$. (Here, one should think of the " $D$ " as standing for "disk.") Thus, we may choose an isomorphism $D \cong \operatorname{Spec}(K[[t]])$ (where $t$ is an indeterminate). Let $D_{\infty} \rightarrow D$ be the ramified covering of infinite degree obtained by adjoining all $t^{\frac{1}{N}}$ (for $N$ a positive integer) to $\mathcal{O}_{D}$. Let $\mathfrak{p} \in X_{M}$ be the prime which is the fiber $X_{m}$ of $X_{M} \rightarrow M$ over $M$. Let $\mathcal{U}_{D}$ be the spectrum of the completion of $\mathcal{O}_{X_{M}}, \mathfrak{p}$. Thus, $\mathcal{U}_{D}$ is the spectrum of a complete discrete valuation ring with residue field equal to $K\left(U_{m}\right)$, the function field of $X_{m}$. Let $\eta_{D}$ (respectively, $\eta_{D_{\infty}}$ ) be the generic point of $D$ (respectively, $D_{\infty}$ ). Let $\mathcal{U}_{\eta_{D}} \stackrel{\text { def }}{=} \mathcal{U}_{D} \times_{D} \eta_{D}$. Thus, $\mathcal{U}_{\eta_{D}}$ is the spectrum of a complete, discretely valued field. In particular, it follows 
from the well-known theory of such fields (see, e.g., [Ser2]) that if $\Gamma_{\mathcal{U}_{\eta_{D}}}$ is the absolute Galois group of this field, then we have an exact sequence

$$
1 \rightarrow \widehat{\mathbf{Z}}(1) \rightarrow \Gamma_{\mathcal{U}_{\eta_{D}}} \rightarrow \Gamma_{K\left(U_{m}\right)} \rightarrow 1
$$

Here the covering corresponding to the $\widehat{\mathbf{Z}}(1)$ is given by adjoining all $t^{\frac{1}{N}}$ (for $N$ a positive integer) - cf. the covering $D_{\infty} \rightarrow D$.

Now let us denote by $\Pi_{\mathcal{U}_{\eta_{D}}}$ the result of replacing the geometric portion (i.e., relative to the morphism $\mathcal{U}_{\eta_{D}} \rightarrow \eta_{D}$ ) of the fundamental group of $\mathcal{U}_{\eta_{D}}$ by its maximal pro- $p$ quotient. Then we get an exact sequence

$$
1 \rightarrow \Delta_{U_{m}} \rightarrow \Pi_{\mathcal{U}_{\eta_{D}}} \rightarrow \Gamma_{\eta_{D}} \rightarrow 1
$$

If we pull this exact sequence back via $\Gamma_{\eta_{D_{\infty}}}=\Gamma_{K} \rightarrow \Gamma_{\eta_{D}}$, we thus get an exact sequence

$$
1 \rightarrow \Delta_{U_{m}} \rightarrow \Pi_{\mathcal{U}_{\eta_{D_{\infty}}}} \rightarrow \Gamma_{\eta_{D_{\infty}}}=\Gamma_{K} \rightarrow 1
$$

Moreover, it is easy to see that this last exact sequence can be identified with

$$
1 \rightarrow \Delta_{U_{m}} \rightarrow \Pi_{U_{m}} \rightarrow \Gamma_{K} \rightarrow 1
$$

On the other hand, if we pull back $\Pi_{Y_{L}} \rightarrow \Gamma_{L}$ via $\Gamma_{K}=\Gamma_{\eta_{D_{\infty}}} \rightarrow \Gamma_{L}$, we get an exact sequence

$$
1 \rightarrow \Delta_{Y_{m}} \rightarrow \Pi_{Y_{m}} \rightarrow \Gamma_{K} \rightarrow 1
$$

Thus, if we pull-back $\theta$ via $\Gamma_{K}=\Gamma_{\eta_{D_{\infty}}} \rightarrow \Gamma_{L}$ to obtain a morphism $\Pi_{U_{\eta_{D_{\infty}}}} \rightarrow \Pi_{Y_{\eta_{D_{\infty}}}}$ (where the subscripted $\eta_{D_{\infty}}$ denotes " $\otimes_{L} \eta_{D_{\infty}}$ ") and compose with " $\pi_{1}$ " of the natural morphism $\mathcal{U}_{\eta_{D_{\infty}}} \rightarrow U_{\eta_{D_{\infty}}}$, we get a continuous homomorphism

$$
\theta_{m}: \Pi_{U_{m}} \rightarrow \Pi_{Y_{m}}
$$

over $\Gamma_{K}$ (where $K$ we regard here as the residue field of $M$ at $m$ ).

Lemma 15.1. The morphism $\theta_{m}$ is surjective.

Proof. It suffices to show that the restriction of $\theta_{m}$ to $\Delta_{U_{m}}$ surjects onto $\Delta_{Y_{m}}=\Delta_{Y}$. In fact, by the basic theory of $p$-groups, it suffices to show that $\theta_{m}$ induces a surjection of $\Delta_{U_{m}}$ onto $H_{Y_{m}}=H_{Y}$. To see this, it suffices to consider (after replacing $M$ by a finite 
étale covering of $M$, and enlarging $K$ if necessary) a finite abelian covering $Y_{L}^{\prime} \rightarrow Y_{L}$ (where $Y_{L}^{\prime} \rightarrow L$ is geometrically connected) of degree a power of $p$ which is $>1$. Let us pull-back this covering to some covering $U_{L}^{\prime} \rightarrow U_{L}$ via $\theta$. Then we must show that the pull-back $\mathcal{U}_{\eta_{D_{\infty}}}^{\prime} \rightarrow \mathcal{U}_{\eta_{D_{\infty}}}$ of this covering to $\mathcal{U}_{\eta_{D_{\infty}}}$ is not the trivial covering. Thus, suppose that it $i s$ the trivial covering. Then the covering $U_{m}^{\prime} \rightarrow U_{m}$ that it induces (cf. the exact sequences above) of $U_{m}$ is again trivial. Since the images of $s_{1}, \ldots, s_{r}$ in $X_{m}$ are disjoint this implies first of all that $U_{L}^{\prime} \rightarrow U_{L}$ is unramified at $x_{1}, \ldots, x_{r}$. But because of the way in which $x_{1}, \ldots, x_{r}$ were chosen, this implies that the covering $Y_{L}^{\prime} \rightarrow Y_{L}$ was obtained from a quotient of $H_{Y} / \operatorname{Im}\left(\theta\left(H_{U}^{P}\right)\right)$. Thus, it follows that $U_{L}^{\prime} \rightarrow U_{L}$ extends to a finite étale covering $X_{L}^{\prime} \rightarrow X_{L}$. Moreover, since $X_{M}$ is smooth over $M$ at $m$, it follows that $X_{L}^{\prime} \rightarrow X_{L}$ is split if and only if the induced $X_{m}^{\prime} \rightarrow X_{m}$ is split. But this $X_{m}^{\prime} \rightarrow X_{m}$ extends the covering $U_{m}^{\prime} \rightarrow U_{m}$ which we already saw to be trivial. Thus, we obtain that $X_{L}^{\prime} \rightarrow X_{L}$, hence $U_{L}^{\prime} \rightarrow U_{L}$ is trivial. Since $\theta$ is surjective, however, this implies that $Y_{L}^{\prime} \rightarrow Y_{L}$ is trivial. This contradiction completes the proof of the Lemma.

Now by the induction hypothesis on the transcendence degree of $L$, it follows that $\theta_{m}$ arises from some geometric morphism $U_{m} \rightarrow Y_{m}$ which is compatible with the action of $\sigma$ on both sides ( $\operatorname{since} \sigma$ is compatible with $\theta$ ). By the valuative criterion for properness, this morphism extends to a morphism $X_{m} \rightarrow Y_{m}$. Moreover, $\sigma$ fixes $s_{0}(m) \in X_{m}(K)$, so $s_{0}(m) \in X_{m}(K)$ is mapped to a fixed point of $Y_{m}(K)$, which is absurd, since $\sigma$ acts on $Y_{m}$ without fixed points. This contradiction completes the proof of the following result:

Lemma 15.2. Let $L$ be a finitely generated extension of $\mathbf{Q}_{p}$. Let $X_{L}$ and $Y_{L}$ be proper hyperbolic curves over $L$. Let $U_{L}$ be the generic point of $X_{L}$. Then any continuous surjective homomorphism $\theta: \Pi_{U_{L}} \rightarrow \Pi_{Y_{L}}$ over $\Gamma_{L}$ necessarily factors through $\Pi_{X_{L}}$.

Now we can conclude that $\theta$ arises geometrically, as follows. Consider the $M$-scheme $\mathcal{H}_{M} \stackrel{\text { def }}{=} \operatorname{Hom}_{M}\left(X_{M}, Y_{M}\right) \rightarrow M$. Since $Y_{M}$ is hyperbolic, it is well-known that $\mathcal{H}_{M}$ is finite and unramified over $M$. (Indeed, that $\mathcal{H}_{M} \rightarrow M$ is unramified (respectively, quasifinite; proper) follows since the pull-back of the tangent bundle of $Y_{m}$ to $X_{m}$ (for any $m \in M$ ) has no global sections (respectively, follows from the Hurwitz formula, which allows one to bound the degree of a morphism $X_{m} \rightarrow Y_{m}$; follows via the same argument as that used in Lemma 8.3 of [Mzk1]).) By shrinking $M$, we may assume that $\mathcal{H}_{M}$ is finite étale over $M$. Then the fact that $\theta_{m}$ arises geometrically (from some $X_{m} \rightarrow Y_{m}$ ) shows that over some finite extension $L^{\prime}$ of $L$, we have a morphism $X_{L^{\prime}} \rightarrow Y_{L^{\prime}}$ that specializes to $X_{m} \rightarrow Y_{m}$. Moreover, if $M^{\prime}$ is the normalization of $M$ in $L^{\prime}$, then since the pro- $p$ geometric fundamental groups of $X_{M^{\prime}}$ and $Y_{M^{\prime}}$ form local systems over $M^{\prime}$, it follows (by checking what happens over the point $m$ ) that the morphism on $\Delta$ 's induced by $X_{L^{\prime}} \rightarrow Y_{L^{\prime}}$ is the same (up to composition with an inner automorphism) as that induced by $\theta$. In particular, the morphism $H_{X} \rightarrow H_{Y}$ induced by $X_{L^{\prime}} \rightarrow Y_{L^{\prime}}$ is the same as that induced by $\theta$. On the other hand, a morphism from $X_{L}$ to $Y_{L}$ (over any field) is determined by the morphism it induces from $H_{X}$ to $H_{Y}$ (cf. the proof of the injectivity part of Theorem 14.1). Thus, since the $H_{X} \rightarrow H_{Y}$ in question is $\Gamma_{L^{-}}$(not just $\Gamma_{L^{\prime}}$ ) equivariant, we obtain 
that $X_{L^{\prime}} \rightarrow Y_{L^{\prime}}$ descends to a $X_{L} \rightarrow Y_{L}$. By repeating this construction (as usual) for all finite étale coverings of $Y_{L}$, we also obtain that the morphism induced on fundamental groups by this $X_{L} \rightarrow Y_{L}$ is the original $\theta$.

That is to say, in summary, we have proven that any $\theta: \Pi_{U_{L}} \rightarrow \Pi_{Y_{L}}$ as in Lemma 15.2 necessarily arises geometrically from some morphism $U_{L} \rightarrow Y_{L}$. Thus, by arguments formally analogous to those of the proofs of Theorem 14.1 and Corollary 14.2, we obtain the following analogue of Corollary 14.2:

Corollary 15.3. Let $K$ be a finitely generated field extension of $\mathbf{Q}_{p}$. Let $X_{K}$ (respectively, $\left.Y_{K}\right)$ be either a hyperbolic curve over $K$ or the spectrum of a one-dimensional function field over $K$. Let $\operatorname{Hom}_{K}^{\operatorname{dom}}\left(X_{K}, Y_{K}\right)$ be the set of dominant $K$-morphisms from $X_{K}$ to $Y_{K}$. Let $\operatorname{Hom}_{\Gamma_{K}}^{\text {open }}\left(\Pi_{X_{K}}, \Pi_{Y_{K}}\right)$ be the set of open, continuous group homomorphisms $\Pi_{X_{K}} \rightarrow \Pi_{Y_{K}}$ over $\Gamma_{K}$, considered up to composition with an inner automorphism arising from $\Delta_{Y}$. Then the natural map

$$
\operatorname{Hom}_{K}^{\text {dom }}\left(X_{K}, Y_{K}\right) \rightarrow \operatorname{Hom}_{\Gamma_{K}}^{\text {open }}\left(\Pi_{X_{K}}, \Pi_{Y_{K}}\right)
$$

is bijective.

It turns out that it is most natural to generalize Corollary 15.3 to the case where the long and unwieldy expression "hyperbolic curve/spectrum of a one-dimensional function field defined over a finitely generated field extension of $\mathbf{Q}_{p}$ " is replaced by the much shorter expression "pro-hyperbolic curve over a sub-p-adic field." Thus, we make the following definition: Let $K$ be a field. Let $X_{K}$ be a $K$-scheme.

Definition 15.4. (i) We shall call a field $K$ a sub-p-adic field if there exists a prime number $p$, together with a finitely generated field extension $L$ of $\mathbf{Q}_{p}$ such that $K$ is isomorphic to a subfield of $L$.

(ii) We shall call $X_{K}$ a hyperbolic pro-curve (over $K$ ) if $X_{K}$ can be written as the projective limit of a projective system of hyperbolic curves over $K$ such that all the transition morphisms in the projective system are birational.

Thus, the following are all examples of sub-p-adic fields:

(1) finitely generated (in particular, finite) extensions of $\mathbf{Q}_{p}$

(2) number fields (i.e., finite extensions of $\mathbf{Q}$ )

(3) the subfield of $\overline{\mathbf{Q}}$ which is the composite of all number fields of degree $\leq n$ over $\mathbf{Q}$ (for some fixed integer $n$ ). 
Another way to think of a pro-hyperbolic curve $X_{K}$ is as the result of removing some set (possibly infinite, possibly empty) of closed points from some hyperbolic curve. In particular, the notion of a "hyperbolic pro-curve" generalizes both the notion of a hyperbolic curve and the case of the spectrum of a function field of dimension one. Then we have the following result:

Corollary 15.5. Let $K$ be sub-p-adic. Let $X_{K}$ (respectively, $Y_{K}$ ) be a hyperbolic pro-curve over $K$. Let $\operatorname{Hom}_{K}^{\text {dom }}\left(X_{K}, Y_{K}\right)$ be the set of dominant $K$-morphisms from $X_{K}$ to $Y_{K}$. Let $\operatorname{Hom}_{\Gamma_{K}}^{\text {open }}\left(\Pi_{X_{K}}, \Pi_{Y_{K}}\right)$ be the set of open, continuous group homomorphisms $\Pi_{X_{K}} \rightarrow \Pi_{Y_{K}}$ over $\Gamma_{K}$, considered up to composition with an inner automorphism arising from $\Delta_{Y}$. Then the natural map

$$
\operatorname{Hom}_{K}^{\text {dom }}\left(X_{K}, Y_{K}\right) \rightarrow \operatorname{Hom}_{\Gamma_{K}}^{\text {open }}\left(\Pi_{X_{K}}, \Pi_{Y_{K}}\right)
$$

is bijective.

Proof. First, let us observe that if $K$ is a finitely generated field extension of $\mathbf{Q}_{p}$, then Corollary 15.5 follows immediately from Corollary 15.3 by arguments formally analogous to those of the proofs of Theorem 14.1 and Corollary 14.2. Thus, the only (slightly) "new" phenomenon here is the fact that we allow $K$ to be a subfield of a finitely generated extension of $\mathbf{Q}_{p}$. The argument for such subfields is as follows: Let $K$ be a subfield of a finitely generated extension field $L$ of $\mathbf{Q}_{p}$. Suppose that we have been given $X_{K}$ and $Y_{K}$ as in the statement of Corollary 15.5, as well as an open $\theta: \Pi_{X_{K}} \rightarrow \Pi_{Y_{K}}$ over $\Gamma_{K}$. Let us denote by $\theta_{\text {geom }}$ the morphism $\Delta_{X} \rightarrow \Delta_{Y}$ induced by $\theta$ on geometric fundamental groups. By base-changing to $L$ and applying the Corollary 15.5 over $L$, we obtain that there exists a morphism $\phi_{L}: X_{L} \rightarrow Y_{L}$ whose induced morphism on geometric fundamental groups coincides with that defined by $\theta$. On the other hand, since morphisms between hyperbolic curves (hence also hyperbolic pro-curves), clearly have no moduli (cf. the fact that the scheme $\mathcal{H}_{M}$ of the discussion preceding Corollary 15.3 was finite and unramified over $M$ ), it follows that $\phi_{L}$ descends to a finite Galois extension $K^{\prime}$ of $K$. Thus, we have a morphism $\phi_{K^{\prime}}: X_{K^{\prime}} \rightarrow Y_{K^{\prime}}$. It remains to descend $\phi_{K^{\prime}}$ to $K$. But this follows from the fact that if $\sigma \in \operatorname{Gal}\left(K^{\prime} / K\right)$, then conjugating $\phi_{K^{\prime}}$ by $\sigma$ gives a morphism $\phi_{K^{\prime}}^{\sigma}: X_{K^{\prime}} \rightarrow Y_{K^{\prime}}$ whose induced morphism on geometric fundamental groups is the result of conjugating $\theta_{\text {geom }}$ by $\sigma$. On the other hand, $\theta_{\text {geom }}$ arises from $\theta$ which lies over $\Gamma_{K}$, so $\theta_{\text {geom }}$ is fixed (up to composition with an inner automorphism defined by an element of $\Delta_{Y}$ ) by conjugation by $\sigma$. Thus, $\phi_{K^{\prime}}$ and $\phi_{K^{\prime}}^{\sigma}$ induce the same morphism on geometric fundamental groups, hence coincide (cf. the argument of the discussion preceding Corollary 15.3). This shows that $\phi_{K^{\prime}}$ descends to a morphism $\phi_{K}: X_{K} \rightarrow Y_{K}$. Repeating this construction (as usual - cf. the argument preceding the statement of Theorem 14.1) for coverings of $X_{K}$ and $Y_{K}$ shows that the morphism induced by $\phi_{K}$ on fundamental groups coincides with $\theta$ (up to composition with an inner automorphism defined by an element of $\Delta_{Y}$ ).

Remark. Note that in Corollaries 15.3 and 15.5, in fact, we implicitly used the fact that 
for $K$ as in those Corollaries, $\Gamma_{K}$ is center-free. This may be proven as follows. First, if $K$ is a finite extension of $\mathbf{Q}_{p}$, then we have the following:

Lemma 15.6. If $K$ is a finite extension of $\mathbf{Q}_{p}$, then the group $\Gamma_{K}$ is center-free.

Proof. (The argument given here is "well-known," but was related to the author by A. Tamagawa.) Since one knows explicitly (see, e.g., [Ser2], Chapter IV, §2) the structure of $\operatorname{Gal}\left(K^{\mathrm{tm}} / K\right)$, it is clear that the quotient $\operatorname{Gal}\left(K^{\mathrm{tm}} / K\right)$ of $\Gamma_{K}$ is center-free. Thus, the center of $\Gamma_{K}$ must lie in $\Gamma_{K^{\mathrm{tm}}} \subseteq \Gamma_{K}$. On the other hand, $\Gamma_{K^{\mathrm{tm}}}$ is a pro- $p$ group. Moreover, it follows immediately from the facts that

(1) $H^{2}\left(K, \mathbf{F}_{p}(1)\right)=\mathbf{F}_{p}$;

(2) if $K^{\prime}$ is a finite unramified extension of $K$ of degree $p$ (which always exists), then the natural morphism $H^{2}\left(K, \mathbf{F}_{p}(1)\right) \rightarrow H^{2}\left(K^{\prime}, \mathbf{F}_{p}(1)\right)$ is zero;

that $H^{2}\left(\Gamma_{K^{\mathrm{tm}}}, \mathbf{F}_{p}\right)=0$. But by [Shatz], Chapter III, $\S 3$, Proposition 2.3, this is enough to imply that $\Gamma_{K^{\mathrm{tm}}}$ is free (as a pro- $p$ group), which implies that its center is trivial (see, e.g., [Tama], §1, Propositions 1.1, 1.11).

Next, we consider the case of a finitely generated extension of $\mathbf{Q}_{p}$ :

Lemma 15.7. Suppose that $K_{0}$ is a field of characteristic zero with the property that every open subgroup of $\Gamma_{K_{0}}$ is center-free. Then any finitely generated field extension $K$ of $K_{0}$ also has this property. In particular, if $K$ is a finitely generated field extension of $\mathbf{Q}_{p}$, then $\Gamma_{K}$ is center-free.

Proof. The last statement follows from the first plus Lemma 15.6. Thus, let us prove the first statement. Note that an extension of a center-free group by a center-free group is center-free. Thus, it suffices to prove that if $L$ is a function field (of arbitrary finite dimension) over $\bar{K}_{0}$, then $\Gamma_{L}$ is center-free. Next, note that the projective limit of a projective system of center-free groups in which all the transition morphisms are surjective is center-free. Moreover, as is well-known, $\operatorname{Spec}(L)$ is a projective limit of "hyperbolic Artin neighborhoods" over $\bar{K}_{0}$ (i.e., successive fibrations of hyperbolic curves - see [SGA4], XI 3.3). Thus, it suffices to prove that the fundamental group of such an Artin neighborhood is center-free. But this then reduces to showing that the fundamental group of a hyperbolic curve is center-free, which is well-known (see, e.g., [Tama], §1, Proposition 1.11).

This is already enough for Corollary 15.3. Now we can conclude the result for arbitrary $K$ as in Corollary 15.5 by means of the following Lemma (due to A. Tamagawa): 
Lemma 15.8. Let $K$ be sub-p-adic. Then $\Gamma_{K}$ is center-free.

Proof. Let $K^{\prime}$ be a finitely generated field extension of $\mathbf{Q}_{p}$ that contains $K$. Suppose that $\sigma \in \Gamma_{K}$ lies in the center of $\Gamma_{K}$, but is not equal to the identity. Then there exists a finite Galois extension $L$ of $K$ such that $\sigma$ maps to an element of $\operatorname{Gal}(L / K)$ other than the identity. Write $L=K(\alpha)$, for some $\alpha \in L$. Let $E_{L}$ be an elliptic curve over $L$ with $j$-invariant $\alpha$. Let $X_{L}$ be the complement of " 0 " in $E_{L}$. Thus, $X_{L}$ is a hyperbolic curve over $L$. Let $Y_{L}$ be the result of base-changing $X_{L}$ by $\sigma: L \rightarrow L$. Thus, it follows that $X_{L}$ and $Y_{L}$ are isomorphic as schemes over $K$. Moreover, conjugating by $\sigma$ defines an isomorphism $\Pi_{X_{L}} \cong \Pi_{Y_{L}}$ which lies over $\Gamma_{L}$ because $\sigma$ is in the center of $\Gamma_{K}$. Basechanging this isomorphism to $L^{\prime}$ (the composite of $K^{\prime}$ and $L$ over $K$ ), we get a $\Gamma_{L^{\prime-}}$ isomorphism $\Pi_{X_{L^{\prime}}} \cong \Pi_{Y_{L^{\prime}}}$. By Corollary 15.3 (in fact, really, this follows already from the results of [Mzk2]), we obtain that this isomorphism arises from an $L^{\prime}$-isomorphism of $X_{L^{\prime}}$ with $Y_{L^{\prime}}$. But this implies that the $j$-invariants of the compactifying elliptic curves of $X_{L^{\prime}}$ and $Y_{L^{\prime}}$ are the same, i.e., that $\alpha=\alpha^{\sigma} \in L \subseteq L^{\prime}$, which is absurd. This contradiction completes the proof of the Lemma.

\section{Section 16: Maps From Higher-Dimensional Function Fields to Curves}

Let $K$ be sub-p-adic (cf. Definition 15.4 (i)). Let $U_{K}$ be the spectrum of a function field over $K$. (Note that here, we do not assume that the dimension of the function field is one.) Let $X_{K}$ be a smooth projective model of $U_{K}$ (which exists by [Hiro]). Thus, $U_{K}$ is the generic point of $X_{K}$. Let $n$ be the dimension of $X_{K}$. By abuse of terminology, we shall also say that $n$ is the "dimension of $U_{K}$ over $K$." Since the one-dimensional case has been dealt with previously, we assume here that $n \geq 2$. The purpose of this Section is to prove a result like Corollary 15.5, except for morphisms between $U_{K}$ and a hyperbolic pro-curve.

Let $\mathcal{L}$ be a very ample line bundle on $X_{K}$. Let

$$
V \stackrel{\text { def }}{=} \Gamma\left(X_{K}, \mathcal{L}\right)
$$

Since the dimension of $X_{K}$ is $\geq 2$, and $\mathcal{L}$ is very ample, it follows that $\operatorname{dim}_{K}(V) \geq 3$. Let $W^{\prime} \subseteq V$ be a one-dimensional (over $K$ ) subspace, generated by a section of $\mathcal{L}$ whose zero locus forms a smooth, connected subvariety of $X_{K}$. (Note that by Bertini's theorem (see, e.g., Theorem 6.3, pp. 66, of [Jou]), it is well-known that such a $W^{\prime}$ exists.) Let $W \subseteq V$ be a two-dimensional subspace containing $W^{\prime}$. Choose a basis $\left\{e_{1}, e_{2}\right\}$ for $W$ such that $e_{1} \in W^{\prime}$. Let $e_{3} \in V$ be an element whose image in $V / W$ is nonzero. Let us also assume that the common zero locus in $X_{K}$ of the three sections $e_{1}, e_{2}, e_{3}$ is a subscheme of $X_{K}$ of codimension $\geq 3$. (This can always be achieved by choosing $e_{1}, e_{2}, e_{3}$ sufficiently generically.) Let $K^{\prime}=K(t)$ (where $t$ is an indeterminate). Let $s_{1} \stackrel{\text { def }}{=} t ; s_{2} \stackrel{\text { def }}{=} t^{-1}$. Then we can construct a new two-dimensional subspace $\widetilde{W} \subseteq V_{K^{\prime}} \stackrel{\text { def }}{=} V \otimes_{K} K^{\prime}$, over $K^{\prime}$, as follows: 
We let $\widetilde{W}$ be the subspace generated by the vectors $\widetilde{e}_{1} \stackrel{\text { def }}{=} e_{1}+s_{1} \cdot e_{3}, \widetilde{e}_{2} \stackrel{\text { def }}{=} e_{2}+s_{2} \cdot e_{3}$. Thus, to summarize, we have the following situation:

$$
\begin{aligned}
& W^{\prime} \subseteq \quad W \quad \subseteq K \cdot e_{1}+K \cdot e_{2}+K \cdot e_{3} \subseteq V \\
& \text { \| } \quad \| \text { \| } \\
& K \cdot e_{1} \subseteq K \cdot e_{1}+K \cdot e_{2} \subseteq K \cdot e_{1}+K \cdot e_{2}+K \cdot e_{3} \\
& \widetilde{W}=K^{\prime} \cdot \widetilde{e}_{1}+K^{\prime} \cdot \widetilde{e}_{2} \subseteq V_{K^{\prime}}=V \otimes_{K} K^{\prime} ; \quad K^{\prime}=K(t) \\
& \widetilde{e}_{1}=e_{1}+s_{1} \cdot e_{3} ; \quad \widetilde{e}_{2} \stackrel{\text { def }}{=} e_{2}+s_{2} \cdot e_{3} ; \quad s_{1}=t ; \quad s_{2}=t^{-1}
\end{aligned}
$$

Lemma 16.1. $\quad$ Let $\Omega$ be an algebraically closed field containing $K^{\prime}$. Then there do not exist any one-dimensional subspaces $W^{\prime \prime}$ of $\widetilde{W}_{\Omega} \stackrel{\text { def }}{=} \widetilde{W} \otimes_{K^{\prime}} \Omega$ such that $W^{\prime \prime}$ is defined (as a subspace of $V \otimes_{K} \Omega$ ) over a finite extension of $K$.

Proof. Indeed, if such a $W^{\prime \prime}$ existed, then there would exist elements $a, b, c \in \Omega$ (with $c \neq 0$, and at least one of $a, b$ nonzero), together with $\alpha_{1}, \alpha_{2}, \alpha_{3} \in \bar{K}$ such that

$$
a \cdot \widetilde{e}_{1}+b \cdot \widetilde{e}_{2}=c \cdot\left(\alpha_{1} \cdot e_{1}+\alpha_{2} \cdot e_{2}+\alpha_{3} \cdot e_{3}\right)
$$

By dividing $a$ and $b$ by $c$, we may assume that $c=1$. Then, equating the coefficients of $e_{1}, e_{2}, e_{3}$, we obtain that $a=\alpha_{1} ; b=\alpha_{2} ; s_{1} \cdot a+s_{2} \cdot b=\alpha_{3}$. But this implies that $t, t^{-1}$, and 1 (as elements of $K^{\prime}$ ) are linearly dependent over $\bar{K}$, which is absurd. This completes the proof of the Lemma.

Before proceeding, let us pause to interpret what this Lemma means. Note that the 3 -dimensional $K$-subspace of $V$ generated by $e_{1}, e_{2}, e_{3}$ defines a rational morphism $\psi$ from $X_{K}$ to $Q_{K} \stackrel{\text { def }}{=} \mathbf{P}\left(K \cdot e_{1}+K \cdot e_{2}+K \cdot e_{3}\right)$. Thus, $\psi$ is defined outside of some closed subscheme of $X_{K}$ of codimension $\geq 2$. Moreover, note that the inverse image via $\psi$ of any closed point of $Q_{K}$ is a subscheme of $X_{K}$ of codimension $\geq 2$. (Indeed, this follows from the fact that the common zero locus of $e_{1}, e_{2}, e_{3}$ has codimension $\geq 3$ in $X_{K}$.) Now let $\Omega$ be a finite extension of $K^{\prime}$, and let us consider a one-dimensional (over $\Omega$ ) subspace $W^{\prime \prime}$ of $\widetilde{W}_{\Omega}$. Thus, $W^{\prime \prime}$ corresponds to a line $L_{\Omega} \subseteq Q_{\Omega} \stackrel{\text { def }}{=} Q_{K} \otimes_{K} \Omega$, and the zero locus (in $X_{\Omega} \stackrel{\text { def }}{=} X_{K} \otimes_{K} \Omega$ ) of a nonzero section of $W^{\prime \prime}$ is equal to the closure of $\psi_{\Omega}^{-1}\left(L_{\Omega}\right) \subseteq X_{\Omega}$.

Now we are ready to interpret Lemma 16.1: Namely, I claim that Lemma 16.1 implies that no irreducible component of the (closure of the) divisor $\psi_{\Omega}^{-1}\left(L_{\Omega}\right)$ is defined over $\bar{K}$. 
Indeed, if there were a divisor $D \subseteq X_{K}$ such that $\psi_{\Omega}\left(D_{\Omega}\right) \subseteq L_{\Omega}$, then by spreading out $\Omega$ (respectively, $L_{\Omega}$ ) to a smooth affine curve $C_{K}$ over $K$ (respectively, a family of lines in $Q_{K}$ parametrized by $C_{K}$ ), we obtain that for every closed point $c \in C_{K}$, we have $\psi(D) \subseteq L_{c}$. But since (by Lemma 16.1) $L_{\Omega}$ is not defined over $\bar{K}$, it follows that for two sufficiently generic closed points $c, c^{\prime} \in C_{K}, \operatorname{dim}_{K}\left(L_{c} \bigcap L_{c^{\prime}}\right)=0$, which implies that $D$ is contained in the inverse image of a finite set of closed points of $Q_{K}$. But we saw in the preceding paragraph that the inverse image via $\psi$ of a closed point of $Q_{K}$ is of codimension $\geq 2$. Since $D$ is a divisor, this is absurd. This completes the proof of the claim stated at the beginning of this paragraph.

Next, let us base-change $U_{K}$ and $X_{K}$ to $K^{\prime}$ : this gives rise to $U_{K^{\prime}}$ and $X_{K^{\prime}}$. The two-dimensional subspace $\widetilde{W} \subseteq V_{K^{\prime}}$ defines a rational map from $X_{K^{\prime}}$ to $P_{K^{\prime}} \stackrel{\text { def }}{=} \mathbf{P}(\widetilde{W})$. Let $X_{K^{\prime}}^{\prime} \subseteq X_{K^{\prime}}$ be the complement of the indeterminacy locus of this rational map. Thus, the complement of $X_{K^{\prime}}^{\prime}$ in $X_{K^{\prime}}$ is of codimension $\geq 2$, and, moreover, we obtain a dominant morphism (a "pencil")

$$
\xi: X_{K^{\prime}}^{\prime} \rightarrow P_{K^{\prime}}
$$

Let $\eta_{P}$ be the generic point of $P_{K^{\prime}}$. Let $\bar{\eta}_{P}$ be the spectrum of an algebraic closure of $K\left(\eta_{P}\right)$ (the function field of $P_{K^{\prime}}$ ). Let

$$
F_{\eta_{P}} \subseteq X_{\eta_{P}} \stackrel{\text { def }}{=} X_{K} \times_{K} \eta_{P}
$$

be the divisor which is the zero locus of the section of $\mathcal{L} \otimes_{K} K\left(\eta_{P}\right)$ defined by the generic point $\eta_{P}$ of $P_{K^{\prime}}$. (Thus, $F_{\eta_{P}} \rightarrow \eta_{P}$ is proper.) Put another way, $F_{\eta_{P}}$ is the (closure in $X_{\eta_{P}}$ of the) fiber $\left(\subseteq X_{\eta_{P}}^{\prime} \subseteq X_{\eta_{P}}\right)$ of the morphism $\xi$ over the generic point $\eta_{P} \in P_{K^{\prime}}$ (whence the use of the letter " $F$ "). Since the pencil $P_{K^{\prime}}$ contains $\widetilde{e}_{1}$, which is a genericization of $e_{1}$ (i.e., $e_{1}$ is the specialization of $\widetilde{e}_{1}$ at $s_{1}=t=0$ ), and the zero locus of $e_{1}$ is (geometrically) smooth and connected, it follows that $F_{\eta_{P}}$ will also be geometrically smooth and connected over $\eta_{P}$. Moreover, by the Lefshetz hyperplane theorem (see, e.g., [SGA2]), it follows (since $\operatorname{dim}_{K}\left(X_{K}\right) \geq 2$ ) that the natural morphism

$$
\pi_{1}\left(F_{\bar{\eta}_{P}}\right) \rightarrow \pi_{1}\left(X_{\bar{\eta}_{P}}\right)=\pi_{1}\left(X_{\bar{K}^{\prime}}\right)=\pi_{1}\left(X_{\bar{K}}\right)
$$

is surjective. Let

$$
G_{\eta_{P}} \stackrel{\text { def }}{=} F_{\eta_{P}} \times_{X_{\eta_{P}}} U_{\eta_{P}}=F_{\eta_{P}} \times_{X_{K}} U_{K}
$$

Since $U_{K}$ is a projective limit of dense open subschemes of $X_{K}$, it thus follows that $G_{\eta_{P}}$ is a projective limit of open subschemes of $F_{\eta_{P}}$. Moreover, since $F_{\eta_{P}}$ (thought of as (the closure of) the fiber of the dominant morphism $\xi$ over the generic point $\eta_{P}$ of $P_{K^{\prime}}$ ) contains (as a dense open subset) a scheme which is a projective limit of dense open subschemes of $X_{K^{\prime}}$, we conclude that $G_{\eta_{P}}$ is an integral, nonempty scheme. 
At any rate, we get a natural morphism

$$
\pi_{1}\left(G_{\bar{\eta}_{P}}\right) \rightarrow \pi_{1}\left(U_{\bar{\eta}_{P}}\right)=\pi_{1}\left(U_{\bar{K}^{\prime}}\right)=\pi_{1}\left(U_{\bar{K}}\right)
$$

Now we have the following important

Lemma 16.2. This morphism $\pi_{1}\left(G_{\bar{\eta}_{P}}\right) \rightarrow \pi_{1}\left(U_{\bar{K}}\right)$ is surjective.

Proof. It suffices to take a finite, connected étale covering $\widetilde{U}_{\bar{K}} \rightarrow U_{\bar{K}}$ (of degree $>1$ ), pull it back to a covering $\widetilde{G}_{\bar{\eta}_{P}} \rightarrow G_{\bar{\eta}_{P}}$ over $G_{\bar{\eta}_{P}}$, and show that this pulled back covering can never be split. Indeed, suppose that $\widetilde{G}_{\bar{\eta}_{P}} \rightarrow G_{\bar{\eta}_{P}}$ is split. Now observe that since $\xi$ is dominant, it follows that $F_{\bar{\eta}_{P}} \rightarrow X_{\bar{K}^{\prime}}$ and $G_{\bar{\eta}_{P}} \rightarrow U_{\bar{K}^{\prime}}$ are dominant. In fact, $F_{\eta_{P}} \rightarrow X_{K^{\prime}}$ and $G_{\eta_{P}} \rightarrow U_{K^{\prime}}$ are even birational isomorphisms. Moreover, I claim that the divisors (in $\left.X_{K^{\prime}}\right)$ that were thrown out of $X_{K^{\prime}}$ to create $F_{\eta_{P}}$ - let us call these divisors F-divisors are different from the divisors that were thrown out of $X_{K^{\prime}}$ to create $U_{K^{\prime}}$ - which we shall call $U$-divisors. Indeed, as divisors of $X_{K^{\prime}}=X_{K} \otimes_{K} K^{\prime}$, the $U$-divisors are all defined over finite extensions of $K$, whereas the $F$-divisors (which are just fibers of $\xi$ over closed points of $P_{K^{\prime}}$ ) are, by Lemma 16.1 (see also the interpretation of Lemma 16.1 in the two paragraphs following the proof of Lemma 16.1), never defined over finite extensions of $K$. This proves the claim.

Next, let us observe that the morphism $F_{\bar{\eta}_{P}} \rightarrow F_{\eta_{P}}$ is ramified only over $F$-divisors. Thus, it is unramified over $U$-divisors. Since $G_{\bar{\eta}_{P}} \rightarrow F_{\bar{\eta}_{P}}$ is birational, the fact that $\widetilde{G}_{\bar{\eta}_{P}} \rightarrow G_{\bar{\eta}_{P}}$ splits (hence, in particular, extends to a finite étale covering over $F_{\bar{\eta}_{P}}$ ) thus implies that the original $\widetilde{U}_{\bar{K}} \rightarrow U_{\bar{K}}$ is unramified over the $U$-divisors, i.e., $\widetilde{U}_{\bar{K}} \rightarrow U_{\bar{K}}$ arises from a covering $\widetilde{X}_{\bar{K}} \rightarrow X_{\bar{K}}$. (Here we use "purity of the branch locus" for regular local rings - see, e.g., [SGA2], Exposé X, p. 118, Théorème 3.4.) But then the fact that the pull-back $\widetilde{F}_{\bar{\eta}_{P}} \rightarrow F_{\bar{\eta}_{P}}$ of this covering to $F_{\bar{\eta}_{P}}$ does not split follows from the surjectivity observed immediately before the statement of this Lemma. Thus, since $G_{\bar{\eta}_{P}} \rightarrow F_{\bar{\eta}_{P}}$ is birational, it follows that $\widetilde{G}_{\bar{\eta}_{P}} \rightarrow G_{\bar{\eta}_{P}}$ cannot split either. This contradiction completes the proof of the Lemma.

Now let us assume that we are given a surjective continuous homomorphism over $\Gamma_{K}$

$$
\theta: \Pi_{U_{K}} \rightarrow \Pi_{Y_{K}}
$$

where $Y_{K}$ is a proper hyperbolic curve over $K$. Let $Y_{\eta_{P}} \stackrel{\text { def }}{=} Y_{K} \times_{K} \eta_{P}$. Thus, $Y_{\eta_{P}}$ is a proper hyperbolic curve over $\eta_{P}$. Suppose, moreover, that we know that morphisms like $\theta$ necessarily arise geometrically (i.e., from a dominant morphism $U_{K} \rightarrow Y_{K}$ ) for $U_{K}$ of dimension $<n$. (We shall refer to this assumption as the "Induction Hypothesis." 
Note that by Corollary 15.5, we already know that this induction hypothesis is true for $\operatorname{dim}_{K}\left(U_{K}\right)=1$.) Then the morphism

$$
\Pi_{G_{\eta_{P}}} \rightarrow \Pi_{Y_{K}}
$$

obtained by composing ( " $\pi_{1}$ of") the natural morphism $G_{\eta_{P}} \rightarrow U_{K}$ with $\theta$ lies over $\Gamma_{\eta_{P}} \rightarrow$ $\Gamma_{K}$, and by Lemma 16.2, is such that it induces a surjection between the (maximal pro$p$ quotients of the) respective geometric fundamental groups. Moreover, this morphism naturally defines a morphism

$$
\theta_{G}: \Pi_{G_{\eta_{P}}} \rightarrow \Pi_{Y_{\eta_{P}}}
$$

(since $\Pi_{Y_{\eta_{P}}}$ is the fibered product of $\Pi_{Y_{K}}$ and $\Gamma_{\eta_{P}}$ over $\Gamma_{K}$ ). Thus, to summarize, $\theta_{G}$ is a surjective continuous homomorphism over $\Gamma_{\eta_{P}}$.

Let $U_{\eta_{P}}^{G}$ be the generic point of $G_{\eta_{P}}$. Thus, by composing (" $\pi_{1}$ of") $U_{\eta_{P}}^{G} \rightarrow G_{\eta_{P}}$ with $\theta_{G}$, we obtain a continuous surjective homomorphism

$$
\theta_{U^{G}}: \Pi_{U_{\eta_{P}}^{G}} \rightarrow \Pi_{Y_{\eta_{P}}}
$$

over $\eta_{P}$. Moreover, the dimension of $U_{\eta_{P}}^{G}$ over $\eta_{P}$ is $<n$. Thus, by the induction hypothesis, it follows that $\theta_{U^{G}}$ arises geometrically, from some unique dominant morphism $U_{\eta_{P}}^{G} \rightarrow Y_{\eta_{P}}$. Projecting to $Y_{K^{\prime}}$ then gives a dominant morphism $U_{\eta_{P}}^{G} \rightarrow Y_{K^{\prime}}$.

We would like to observe that this morphism $U_{\eta_{P}}^{G} \rightarrow Y_{K^{\prime}}$ extends to $U_{K^{\prime}}$. Indeed, to see this, observe that $U_{\eta_{P}}^{G}$ and $U_{K^{\prime}}$ are both projective limits of open subsets of the projective $K^{\prime}$-variety $X_{K^{\prime}}$. Moreover, $U_{K^{\prime}}$ can be written as the projective limit of $K^{\prime}$ smooth open subsets of $X_{K^{\prime}}$. Thus, the fact that we get a morphism

$$
U_{K^{\prime}} \rightarrow Y_{K^{\prime}}
$$

follows from the following

Lemma 16.3. $\quad$ Let $L$ be a field; $\mathcal{Y}_{L}$ be a proper, smooth, geometrically connected curve of nonzero genus over $L$; and $\mathcal{Z}_{L}$ be a smooth L-variety. Suppose that we have a rational map $\phi$ from $\mathcal{Z}_{L}$ to $\mathcal{Y}_{L}$. Then $\phi$ is defined over all of $\mathcal{Z}_{L}$.

Proof. This Lemma is a well-known consequence of the classical theory of the Albanese variety (see, e.g., [Lang]): Namely, let $\mathcal{U}_{L} \subseteq \mathcal{Z}_{L}$ be a (nonempty) open over which $\phi$ is defined. Assume (without loss of generality) that $\mathcal{Y}_{L}(L) \neq \emptyset$. Let $\mathcal{A}_{L}$ be the Jacobian of $\mathcal{Y}_{L}$, and let $\mathcal{B}_{L}$ be its dual. Then a point of $\mathcal{Y}_{L}(L)$ defines an embedding $\mathcal{Y}_{L} \hookrightarrow \mathcal{A}_{L}$ which we can compose with $\phi$ to obtain a rational map $\phi_{\mathcal{A}}$ from $\mathcal{Z}_{L}$ to $\mathcal{A}_{L}$. It suffices to show 
that $\phi_{\mathcal{A}}$ extends to a morphism on $\mathcal{Z}_{L}$. But note that the portion of $\phi_{\mathcal{A}}$ which already is a morphism (from $\mathcal{U}_{L}$ to $\mathcal{A}_{L}$ ) defines (by pulling back the Poincaré bundle on $\mathcal{A}_{L} \times_{L} \mathcal{B}_{L}$ ) a line bundle on $\mathcal{U}_{L} \times{ }_{L} \mathcal{B}_{L}$. Since $\mathcal{Z}_{L} \times_{L} \mathcal{B}_{L}$ is a regular scheme, it follows that this line bundle extends to $\mathcal{Z}_{L} \times{ }_{L} \mathcal{B}_{L}$. Then taking the classifying morphism associated to this extended line bundle gives a morphism $\mathcal{Z}_{L} \rightarrow \mathcal{A}_{L}$, as desired.

Let us review what we have done so far. We started with a continuous surjective homomorphism

$$
\theta: \Pi_{U_{K}} \rightarrow \Pi_{Y_{K}}
$$

over $\Gamma_{K}$. Moreover, we have shown that if we base-change $\theta$ from $K$ to $K^{\prime}$ to obtain

$$
\theta_{K^{\prime}}: \Pi_{U_{K^{\prime}}} \rightarrow \Pi_{Y_{K^{\prime}}}
$$

then $\theta_{K^{\prime}}$ arises geometrically from some dominant $U_{K^{\prime}} \rightarrow Y_{K^{\prime}}$. Note, moreover, that this morphism $U_{K^{\prime}} \rightarrow Y_{K^{\prime}}$ is the unique morphism that gives rise to (the geometric portion of) $\theta$. (This follows, for instance, from the inductive hypothesis on $n$. Moreover, this uniqueness also holds, of course, over any finite extension of $K^{\prime}$.) Thus, if we specialize the indeterminate $t \in K^{\prime}$ to some element of a finite extension $L$ of $K$ that is sufficiently generic so that $U_{K^{\prime}} \rightarrow Y_{K^{\prime}}$ specializes to $U_{L} \rightarrow Y_{L}$, and then base-change this $U_{L} \rightarrow Y_{L}$ back up to a morphism $U_{L^{\prime}} \rightarrow Y_{L^{\prime}}$ (where $L^{\prime}$ is the composite of $K^{\prime}$ and $L$ ), then this morphism $U_{L^{\prime}} \rightarrow Y_{L^{\prime}}$ coincides with the morphism obtained by base-changing the original $U_{K^{\prime}} \rightarrow Y_{K^{\prime}}$ via $K^{\prime} \subseteq L^{\prime}$. But this means that the original $U_{K^{\prime}} \rightarrow Y_{K^{\prime}}$ is, in fact, defined over $L$. Finally, since $K^{\prime}$ and $L$ are linearly disjoint over $K$, it follows that the original $U_{K^{\prime}} \rightarrow Y_{K^{\prime}}$ is defined over $K$. Thus, we get a morphism $U_{K} \rightarrow Y_{K}$ which clearly induces the original $\theta$ (since, for instance, $\Pi_{U_{K^{\prime}}} \rightarrow \Pi_{U_{K}}$ is surjective).

Let us step back now and take stock of what we have done so far in this Section. We started with $U_{K}$, the spectrum of a function field over $K$, and a proper hyperbolic curve $Y_{K}$ over $K$. Then given any surjective continuous homomorphism

$$
\theta: \Pi_{U_{K}} \rightarrow \Pi_{Y_{K}}
$$

over $\Gamma_{K}$, we showed that $\theta$ necessarily arises geometrically. Now let us pause for a definition:

Definition 16.4. Let $\mathcal{Q}_{K}$ be a $K$-scheme. We shall call $\mathcal{Q}_{K}$ a smooth pro-variety if it is the projective limit of a projective system of smooth (geometrically connected) varieties over $K$ such that the transition morphisms are all birational.

Now Lemma 16.3, plus the techniques of the proofs of Theorem 14.1 and Corollary 14.2 show that we have, in fact, proven the following (our first main theorem - Theorem $A$ in the Introduction): 
Theorem 16.5. $\quad$ Let $K$ be sub-p-adic (cf. Definition 15.4 (i)). Let $X_{K}$ (respectively, $Y_{K}$ ) be a smooth pro-variety (respectively, hyperbolic pro-curve) over $K$. Let $\operatorname{Hom}_{K}^{\operatorname{dom}}\left(X_{K}, Y_{K}\right)$

the set of dominant $K$-morphisms from $X_{K}$ to $Y_{K}$. Let $\operatorname{Hom}_{\Gamma_{K}}^{\text {open }}\left(\Pi_{X_{K}}, \Pi_{Y_{K}}\right)$ be the set of open, continuous group homomorphisms $\Pi_{X_{K}} \rightarrow \Pi_{Y_{K}}$ over $\Gamma_{K}$, considered up to composition with an inner automorphism arising from $\Delta_{Y}$. Then the natural map

$$
\operatorname{Hom}_{K}^{\text {dom }}\left(X_{K}, Y_{K}\right) \rightarrow \operatorname{Hom}_{\Gamma_{K}}^{\text {open }}\left(\Pi_{X_{K}}, \Pi_{Y_{K}}\right)
$$

is bijective.

Remark. Finally, we make the following important observation:

Note that given any pro-p result such as Theorem 16.5, one can always immediately derive a corresponding profinite result from it.

(Here by "corresponding profinite result," we mean the same result, except that "П"

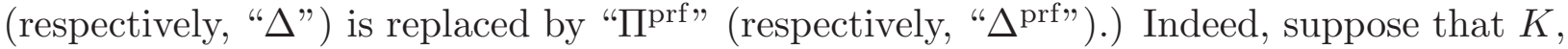
$X_{K}$, and $Y_{K}$ are as in the statement of Theorem 16.5, and let

$$
\theta: \Pi_{X_{K}}^{\mathrm{prf}}=\pi_{1}\left(X_{K}\right) \rightarrow \Pi_{Y_{K}}^{\mathrm{prf}}=\pi_{1}\left(Y_{K}\right)
$$

be an open homomorphism over $\Gamma_{K}$. Then note that $\theta$ immediately induces an open homomorphism $\theta_{p}: \Pi_{X_{K}} \rightarrow \Pi_{Y_{K}}$. Applying Theorem 16.5 shows that $\theta_{p}$ arises from some $\phi: X_{K} \rightarrow Y_{K}$. Thus, it remains only to show that the morphism induced by $\phi$ on fundamental groups coincides with $\theta$. But this follows from the argument preceding Theorem 14.1: namely, we consider an arbitrary finite étale covering $Y_{K}^{\prime} \rightarrow Y_{K}$. Pulling this covering back via $\theta$ gives a finite étale covering $X_{K}^{\prime} \rightarrow X_{K}$. Moreover, $\theta$ induces a morphism between the full profinite fundamental groups of $X_{K}^{\prime}$ and $Y_{K}^{\prime}$. Next, observe that this morphism gives us a $\phi^{\prime}: X_{K}^{\prime} \rightarrow Y_{K}^{\prime}$ (by the same argument as that used to construct $\phi$ from $\theta$ ) which (by naturality of the constructions involved) lies over $\phi$. Thus, if we continue this procedure for arbitrary finite étale coverings, the well-known correspondence between fundamental groups and categories of étale coverings shows that the morphism induced by $\phi$ on fundamental groups coincides with $\theta$ up to composition with an inner automorphism induced by an element of $\Delta_{Y}^{\mathrm{prf}}$. This completes the proof of the "profinite analogue of Theorem 16.5."

\section{Section 17: Maps Between Higher-Dimensional Function Fields}

Let $K$ be sub-p-adic (cf. Definition 15.4 (i)). Let $L$ and $M$ be function fields (of arbitrary dimension) over $K$. (Thus, in particular, we assume here that $K$ is algebraically closed in $L$ and $M$ ). We denote by $\Gamma_{L}$ and $\Gamma_{M}$ the absolute Galois groups of $L$ and 
$M$, respectively. In this Section, we would like to show how to derive a result (Theorem 17.1, which is stated as Theorem $B$ in the Introduction) like Theorem 16.5 for morphisms between $L$ and $M$ (over $K$ ).

Theorem 17.1. $\quad$ Let $K$ be sub-p-adic (cf. Definition 15.4(i)). Let $L$ and $M$ be function fields of arbitrary dimension over $K$. Let $\operatorname{Hom}_{K}(\operatorname{Spec}(L), \operatorname{Spec}(M))$ be the set of $K$-morphisms from $M$ to L. Let $\operatorname{Hom}_{\Gamma_{K}}^{\text {open }}\left(\Gamma_{L}, \Gamma_{M}\right)$ be the set of open, continuous group homomorphisms $\Gamma_{L} \rightarrow \Gamma_{M}$ over $\Gamma_{K}$, considered up to composition with an inner automorphism arising from $\operatorname{Ker}\left(\Gamma_{M} \rightarrow \Gamma_{K}\right)$. Then the natural map

$$
\operatorname{Hom}_{K}(\operatorname{Spec}(L), \operatorname{Spec}(M)) \rightarrow \operatorname{Hom}_{\Gamma_{K}}^{\text {open }}\left(\Gamma_{L}, \Gamma_{M}\right)
$$

is bijective.

Proof. First, recall (cf. the Remark following Theorem 16.5) that any pro- $p$ result such as Theorem 16.5 always implies a corresponding profinite result. Now we use induction on the transcendence degree - which we shall henceforth denote by $\operatorname{dim}_{K}(M)$ - of $M$ over $K$. When $\operatorname{dim}_{K}(M)=0$, the result is vacuous. When $\operatorname{dim}_{K}(M)=1$, the result follows from the profinite version of Theorem 16.5; thus, we may assume that $\operatorname{dim}_{K}(M)>1$. Now suppose that we know Theorem 17.1 to be true for maps to functions fields of transcendence degree $<\operatorname{dim}_{K}(M)$. Suppose that we are given an open continuous homomorphism

$$
\theta: \Gamma_{L} \rightarrow \Gamma_{M}
$$

over $\Gamma_{K}$. Observe that just as previously (e.g., in the proof of Theorem 14.1), we can assume without loss of generality that $\theta$ is surjective.

Next, observe that there exists a function field $P \subseteq M$ such that $0<\operatorname{dim}_{K}(P)<$ $\operatorname{dim}_{K}(M)$. Moreover, we may assume that $P$ is algebraically closed in $M$. Thus, we get a surjection $\Gamma_{M} \rightarrow \Gamma_{P}$. Composing $\theta$ with this surjection, we obtain a surjection $\theta_{P}: \Gamma_{L} \rightarrow \Gamma_{P}$, which, by the induction hypothesis on $\operatorname{dim}_{K}(M)$, we know arises geometrically from some $P \hookrightarrow L$ (over $K$ ). Moreover, since $\theta_{P}$ is surjective, it follows that $P$ is algebraically closed in $L$. Thus, we may regard $L$ and $M$ as function fields over $P$. Moreover, $\operatorname{dim}_{P}(M)<\operatorname{dim}_{K}(M)$. Thus, since $\theta: \Gamma_{L} \rightarrow \Gamma_{M}$ is (by the definition of $\theta_{P}$ ) a morphism over $\Gamma_{P}$, it follows from the induction hypothesis on $\operatorname{dim}_{K}(M)$ that $\theta$ arises from some $M \hookrightarrow L$ over $P$ (hence also over $K$ ). This completes the proof of the Theorem.

Remark. Note that in this case, we needed to work with profinite (not pro- $p$ ) fundamental groups because the operation of taking the maximal pro- $p$ quotient is not well-behaved with respect to fibrations: i.e., if we had replaced $\operatorname{Ker}\left(\Gamma_{M} \rightarrow \Gamma_{K}\right)$ with its maximal pro- $p$ quotient, we would run into trouble because it is not clear that the maximal pro- $p$ quotient of $\operatorname{Ker}\left(\Gamma_{M} \rightarrow \Gamma_{P}\right)$ injects into the maximal pro- $p$ quotient of $\operatorname{Ker}\left(\Gamma_{M} \rightarrow \Gamma_{K}\right)$. 
Also, we needed to work with function fields (as opposed to varieties) because, if, for instance, $\operatorname{Spec}(L)$ and $\operatorname{Spec}(P)$ had been varieties, the fact that the geometric (over $K$ ) fundamental group of $\operatorname{Spec}(L)$ surjects onto that of $\operatorname{Spec}(P)$ does not necessarily imply that the morphism $\operatorname{Spec}(L) \rightarrow \operatorname{Spec}(P)$ has geometrically connected fibers. Moreover, if the generic geometric fiber of $\operatorname{Spec}(L) \rightarrow \operatorname{Spec}(P)$ has several distinct connected components, it is not necessarily the case that the fundamental group of any of these connected components surjects onto the geometric (over $P$ ) fundamental group of $\operatorname{Spec}(M)$. Indeed, by replacing $\operatorname{Spec}(M)$ by a smooth projective variety $Y_{K}$ (of dimension $\geq 3$ ), and $\operatorname{Spec}(L)$ by the result - call it $X_{K}$ - of cutting this variety with a generic hyperplane section, then the inclusion $X_{K} \hookrightarrow Y_{K}$ induces an isomorphism on geometric fundamental groups despite the fact that $X_{K}$ is not isomorphic to $Y_{K}$. Moreover, note that such a counterexample to a "variety version" of Theorem 17.1 exists even if $Y_{K}$ is "hyperbolic" (say, a product of proper hyperbolic curves), in which case, one would expect $X_{K}$ (at least if the ample line bundle used to cut $Y_{K}$ to form $X_{K}$ has sufficiently high degree) to be "quite hyperbolic," as well.

\section{Section 18: Truncated Fundamental Groups}

If $\Delta$ is a topological group, let us introduce the following notation: $\Delta\{0\} \stackrel{\text { def }}{=} \Delta$; for $i \geq 1, \Delta\{i\} \stackrel{\text { def }}{=}[\Delta\{i-1\}, \Delta\{i-1\}]$. Also, let us write " $\Pi^{i} "$ (respectively, " $\Delta$ ") for $\Pi / \Delta\{i\}$ (respectively, $\Delta / \Delta\{i\}$ ). The purpose of this Section is to observe that much of the theory of this paper continues to hold to a large extent even when we consider morphisms not between the full $\Pi$ 's, but between certain truncated versions of the $\Pi$ 's. Our first main result is the following:

Theorem 18.1. $\quad$ Let $K$ be sub-p-adic (cf. Definition 15.4 (i)). Let $X_{K}$ be a smooth variety over $K$. Let $Y_{K}$ be a hyperbolic curve over $K$. Let $n \geq 5$. Then every continuous open homomorphism

$$
\theta: \Pi_{X_{K}}^{n} \rightarrow \Pi_{Y_{K}}^{n}
$$

over $\Gamma_{K}$ induces a dominant morphism $\mu: X_{K} \rightarrow Y_{K}$ whose induced morphism on fundamental groups coincides (up to composition with an inner automorphism arising from $\left.\Delta_{Y_{K}}\right)$ with the morphism $\Delta_{X_{K}}^{n-3} \rightarrow \Delta_{Y_{K}}^{n-3}$ defined by considering $\theta$ "modulo $\Delta\{n-3\}$."

Proof. First let us consider the case where: (i) $K$ is a finite extension of $\mathbf{Q}_{p}$; (ii) $X_{K}$ is a curve; (iii) $Y_{K}$ is a non-hyperelliptic proper hyperbolic curve; (iv) $n=3$. Then note that the theory of Sections 1 through 5 manifestly only involves $\Delta_{X_{K}}^{2}$. It thus follows immediately (via the help of a technical lemma - Lemma 18.4 below - necessary in order to assure that Lemma 7.3 goes through in the truncated context) that to prove Proposition 7.4 , we really only used $\Delta_{Y_{\eta_{S}}^{\prime}}^{2}$. Thus, in summary, as long as the covering $Y_{\eta_{S}}^{\prime} \rightarrow Y_{\eta_{S}}$ (in 
the notation of the discussion preceding Proposition 7.4) arises from an open subgroup of $\Pi_{Y_{K}}^{1}$, we can apply Proposition 7.4 to $Y_{\eta_{S}}^{\prime}$ to conclude that $Y_{\eta_{S}}^{\prime}$ admits a line bundle of degree prime to $p$.

Now we would like to conclude the "preservation of relations." Note that Section 11 manifestly only involves the abelianization of the geometric fundamental group. Moreover, Section 12 is just formal manipulation. In Section 13, the only thing from Sections 1 through 7 that is used is Proposition 7.4 (whose applicability under the present circumstances was discussed in the preceding paragraph). Thus, by using Propositions 7.4 and 8.1, Lemma 8.2, and the theory of Section 9, we may conclude "preservation of relations" for the morphism $H^{0}\left(Y_{K}, \omega_{Y_{K} / K}\right) \rightarrow H^{0}\left(X_{K}, \omega_{X_{K} / K}\right)$ induced by $\Delta_{X_{K}}^{1} \rightarrow \Delta_{Y_{K}}^{1}$. (Note, however, that this time, in the application of Section 9 , we take for our tower of coverings " $X_{L}^{n}$ " not the coverings corresponding to the $p$-derivates of the whole geometric fundamental group $\Delta$, but rather the $p$-derivates of $\Delta^{1}$ - which is enough to carry out the argument of Section 9.) Hence we get a morphism $\mu: X_{K} \rightarrow Y_{K}$.

Now let us lift the hypothesis that $n=3$. Then we obtain the following: If $Y_{K}^{\prime} \rightarrow Y_{K}$ is a covering arising from an open subgroup of $\Pi_{Y_{K}}^{n-3}$, and $X_{K}^{\prime} \rightarrow X_{K}$ is a connected component of the pull-back of this covering to $X_{K}$ via $\theta$, then we get a natural morphism $\mu^{\prime}: X_{K}^{\prime} \rightarrow Y_{K}^{\prime}$. Moreover, the naturality of the construction of this morphism means that it always lies over the morphism $X_{K} \rightarrow Y_{K}$. Thus, it follows by the usual argument (i.e., the one preceding Theorem 14.1) that the morphism induced by $\mu$ on fundamental groups is equal to $\theta$ modulo $\Delta\{n-3\}$ (up to composition with an inner automorphism arising from $\left.\Delta_{Y_{K}}\right)$.

So far we have not used that $n \geq 5$. (In fact, we have only used that $n \geq 3$.) The purpose of assuming that $n \geq 5$ is to lift hypothesis (iii) (in the first paragraph of this proof). Namely, given any hyperbolic curve $Y_{K}$, it is elementary to show that there always exists a covering $Y_{K}^{\prime} \rightarrow Y_{K}$ defined by an open subgroup of $\Pi_{Y_{K}}^{2}$ such that the compactification $\bar{Y}_{K}^{\prime}$ of $Y_{K}^{\prime}$ is hyperbolic and non-hyperelliptic, and such that $\bar{Y}_{K}^{\prime} \rightarrow \bar{Y}_{K}$ has arbitrarily large (specified) ramification over all the points of $\bar{Y}_{K}-Y_{K}$. Thus, we get a map $X_{K}^{\prime} \rightarrow \bar{Y}_{K}^{\prime}$ which descends to a map $X_{K} \rightarrow \bar{Y}_{K}$. Moreover, the fact that $X_{K}^{\prime} \rightarrow X_{K}$ is étale, while $\bar{Y}_{K}^{\prime} \rightarrow \bar{Y}_{K}$ is ramified over the points of $\bar{Y}_{K}-Y_{K}$ (with ramification indices arbitrarily large) implies that the map $X_{K} \rightarrow \bar{Y}_{K}$ factors through $Y_{K}$. Thus, we get a map $X_{K} \rightarrow Y_{K}$, as desired. Then arguing as in the preceding paragraph completes the proof, albeit still under the assumptions (i) and (ii) (of the first paragraph of the proof).

The extension to the case of fields $K$ that are subfields of finitely generated extensions of $\mathbf{Q}_{p}$ then follows via the same "specialization argument" as that employed previously in the nontruncated case (following Lemma 15.2). Thus, we can also lift assumption (i). Finally, the "cutting with a hyperplane argument" of Section 16 extends immediately to the truncated case. This allows us to lift assumption (ii), thus completing the proof of the Theorem.

Theorem 18.2. $\quad$ Let $K$ be a subfield of a finitely generated field extension of $\mathbf{Q}_{p}$. Let $X_{K}$ be a smooth pro-variety over $K$. Let $Y_{K}$ be a hyperbolic pro-curve over $K$. Let $n_{0}^{\prime}$ be 
the minimum transcendence degree over $\mathbf{Q}_{p}$ of all finitely generated field extensions of $\mathbf{Q}_{p}$ that contain $K$. Let $n_{0}^{\prime \prime}$ be the transcendence degree over $K$ of the function field of $X_{K}$. Let $n_{0} \stackrel{\text { def }}{=} n_{0}^{\prime}+2\left(n_{0}^{\prime \prime}-1\right)+1$.

Let $n \geq 3 n_{0}+5$. Then every continuous open homomorphism

$$
\theta: \Pi_{X_{K}}^{n} \rightarrow \Pi_{Y_{K}}^{n}
$$

over $\Gamma_{K}$ induces a dominant morphism $\mu: X_{K} \rightarrow Y_{K}$ whose induced morphism on fundamental groups coincides (up to composition with an inner automorphism arising from $\Delta_{Y_{K}}$ ) with the morphism $\Delta_{X_{K}}^{n-3-3 n_{0}} \rightarrow \Delta_{Y_{K}}^{n-3-3 n_{0}}$ defined by considering $\theta$ "modulo $\Delta\left\{n-3-3 n_{0}\right\} . "$

Proof. The reason for the inclusion of the extra " $3 n_{0}$ " (i.e., a "price" of three steps for every additional transcendence degree that is used, plus an extra "tax" of three steps for allowing the prefix "pro") is the following: in order to reduce to the situation discussed in the proof of Theorem 18.1, we need to show (in the present "pro" context) that inertia groups are annihilated. Moreover, to apply the first "inertia annihilation argument" (at the beginning of Section 14), we needed to know that after one passes to some covering $Y_{K}^{\prime} \rightarrow Y_{K}$ arising from an open subgroup of $\Pi_{Y_{K}}^{n-3}$ (i.e., we wish to apply the arguments of Theorem 18.1 for $n-3)$, one knows "preservation of relations" for $Y_{K}^{\prime}$. Thus, already one needs some extra padding - to the tune of three steps (necessary, as we saw in the first two paragraphs of the proof of Theorem 18.1, to derive "preservations of relations" for $Y_{K}^{\prime}$ ). (This accounts for the 1 in the definition of $n_{0}$.) Moreover, each time one adds a transcendence degree, one needs to apply the "inertia annihilation argument" of the first half of Section 15. Thus, by the same line of reasoning, one needs extra padding consisting of three steps. (This accounts for the $n_{0}^{\prime}$ in the definition of $n_{0}$.) In Section 16, one uses not only the transcendence degrees inherent in $K$, but also $2\left(n_{0}^{\prime \prime}-1\right)$ auxiliary transcendence degrees - here the " $2=1+1$ " comes from the transcendence degree of " $K$ " over " $K$," plus the transcendence degree of the pencil " $\xi$." (This accounts for the $2\left(n_{0}^{\prime \prime}-1\right.$ ) in the definition of $n_{0}$.) Finally, we remark that although in Sections 14, 15, and 16, we assumed that the morphism of fundamental groups " $\theta$ " was surjective, it is easy to see that this assumption is merely cosmetic, i.e., is inessential and serves only to simplify the discussion. This completes the proof of the Theorem.

Remark. Thus, the essential difference between Theorems 18.1 and 18.2 is that in Theorem 18.2, we allow "pro-objects," at the cost of having to apply "annihilation of inertia" arguments, which require us to use larger quotients of $\Delta_{X_{K}}, \Delta_{Y_{K}}$ (i.e., each application of annihilation of inertia costs three units of " $n$ ").

Remark. We do not mean to pretend that the estimates in the above two Theorems (e.g., the "5's," " $n_{0}$," etc.) are the best possible. Especially if one is willing to add hypotheses 
to $Y_{K}$, it should not be so difficult to improve these estimates. The point of the above two Theorems is simply to illustrate the principle involved.

We conclude this Section with two technical lemmas that were used in the proofs of the above two Theorems.

Lemma 18.3. Let $X_{K}$ be a proper hyperbolic curve over a field $K$ of characteristic zero. Let $\Delta_{X}$ be (as usual) the maximal pro-p quotient of its geometric fundamental group. Let $\Xi_{X} \stackrel{\text { def }}{=} \Delta_{X} /\left[\Delta_{X},\left[\Delta_{X}, \Delta_{X}\right]\right]$. Then the natural morphism

$$
H^{2}\left(\Xi_{X}, \mathbf{Z}_{p}(1)\right)^{\Gamma_{K}} \rightarrow H^{2}\left(\Delta_{X}, \mathbf{Z}_{p}(1)\right)^{\Gamma_{K}}
$$

(induced by the quotient map $\Delta_{X} \rightarrow \Xi_{X}$ ) is surjective. Here, the superscripted " $\Gamma_{K}$ " denotes "the submodule of $\Gamma_{K}$-invariants."

Proof. Let us first consider the morphism

$$
H^{2}\left(\Xi_{X}, \mathbf{Z}_{p}(1)\right) \rightarrow H^{2}\left(\Delta_{X}, \mathbf{Z}_{p}(1)\right)
$$

To do this, we shall use the Hochschild-Serre spectral sequence for the quotient $\Xi_{X} \rightarrow H_{X}$ (where, as usual, we write $H_{X}$ for the abelianization of $\Delta_{X}$ ). Write $\Psi_{X} \subseteq_{X} \Xi_{X}$ for the kernel of $\Xi_{X} \rightarrow H_{X}$. Thus, $\Psi_{X}$ may be identified with a certain well-understood (cf. Lemma 3.1) quotient of $\wedge^{2} H_{X}$. Consideration of the $E_{2}$-term of this spectral sequence shows that there is a natural $\Gamma_{K^{-}}$-equivariant injection of the cokernel of the natural morphism (induced by the quotient $\wedge^{2} H_{X} \rightarrow \Psi_{X}$ )

$$
H^{1}\left(\Psi_{X}, \mathbf{Z}_{p}(1)\right)=\operatorname{Hom}_{\mathbf{Z}_{p}}\left(\Psi_{X}, \mathbf{Z}_{p}(1)\right) \rightarrow H^{2}\left(H_{X}, \mathbf{Z}_{p}(1)\right)=\operatorname{Hom}_{\mathbf{Z}_{p}}\left(\wedge^{2} H_{X}, \mathbf{Z}_{p}(1)\right)
$$

into $H^{2}\left(\Xi_{X}, \mathbf{Z}_{p}(1)\right)$. (In other words, the above morphism is the differential (from $E_{2}^{0,1}$ to $\left.E_{2}^{2,0}\right)$ of the " $E_{2}^{p q}=H^{p}\left(H_{X}, H^{q}\left(\Psi_{X}, \mathbf{Z}_{p}(1)\right)\right)$ "-term of the spectral sequence.) Moreover, it follows from Lemma 3.1 that the cokernel of this natural morphism is precisely the quotient of $H^{2}\left(H_{X}, \mathbf{Z}_{p}(1)\right)=\left(\wedge^{2} H^{1}\left(H_{X}, \mathbf{Z}_{p}\right)\right)(1)$ given by the (surjective) cup-product $\operatorname{map}\left(\wedge^{2} H^{1}\left(H_{X}, \mathbf{Z}_{p}\right)\right)(1) \rightarrow H^{2}\left(\Delta_{X}, \mathbf{Z}_{p}\right)(1)$. Thus, in summary, we have a $\Gamma_{K}$-equivariant diagram:

$$
H^{2}\left(\Xi_{X}, \mathbf{Z}_{p}(1)\right) \supseteq \operatorname{Image}\left(H^{2}\left(H_{X}, \mathbf{Z}_{p}(1)\right)\right) \rightarrow H^{2}\left(\Delta_{X}, \mathbf{Z}_{p}(1)\right)
$$

in which the arrow is bijective. This implies the assertion stated in the Lemma. 
Lemma 18.4. Let $K, X_{K}, \Delta_{X}$, and $\Xi_{X}$ be as in Lemma 18.3. Since the kernel of $\Delta_{X} \rightarrow \Xi_{X}$ is a normal subgroup not only of $\Delta_{X}$, but also of $\Pi_{X_{K}}$, write $\Pi_{X_{K}} \rightarrow \Pi_{X_{K}}^{\Xi}$ for the quotient of $\Pi_{X_{K}}$ by this subgroup. Then the natural morphisms

$$
H^{2}\left(\Pi_{X_{K}}^{\Xi}, \mathbf{Z}_{p}(1)\right) \rightarrow H^{2}\left(\Pi_{X_{K}}, \mathbf{Z}_{p}(1)\right)
$$

and

$$
H^{2}\left(\Pi_{X_{K}} \times_{\Gamma_{K}} \Pi_{X_{K}}^{\Xi}, \mathbf{Z}_{p}(1)\right) \rightarrow H^{2}\left(\Pi_{X_{K}} \times_{\Gamma_{K}} \Pi_{X_{K}}=\Pi_{X_{K} \times_{K} X_{K}}, \mathbf{Z}_{p}(1)\right)
$$

are surjective. In particular, if $\eta \in H^{2}\left(\Pi_{X_{K} \times_{K} X_{K}}, \mathbf{Z}_{p}(1)\right)$ denotes the first Chern class of the diagonal in $X_{K} \times_{K} X_{K}$, then $\eta$ lies in the image of $H^{2}\left(\Pi_{X_{K}} \times_{\Gamma_{K}} \Pi_{X_{K}}^{\Xi}, \mathbf{Z}_{p}(1)\right)$.

Proof. The assertions of this Lemma follow by considering the consequences of the surjectivity assertion of Lemma 18.3 for the Hochschild-Serre spectral sequences associated to $\Pi_{X_{K}}^{\Xi} \rightarrow \Gamma_{K} ; \Pi_{X_{K}} \rightarrow \Gamma_{K} ; \Pi_{X_{K}} \times_{\Gamma_{K}} \Pi_{X_{K}}^{\Xi} \rightarrow \Gamma_{K} ;$ and $\Pi_{X_{K}} \times_{\Gamma_{K}} \Pi_{X_{K}} \rightarrow \Gamma_{K}$.

\section{Section 19: Injectivity Result}

In this Section, we prove the following "pro- $p$ injectivity part of the so-called Section Conjecture":

Theorem 19.1. $\quad$ Let $K$ be sub-p-adic (cf. Definition 15.4(i)). Let $X_{K}$ be a hyperbolic curve over $K$. Let $X_{K}(K)$ be the set of $K$-valued points of $X_{K}$. Let $\operatorname{Sect}\left(\Gamma_{K}, \Pi_{X_{K}}\right)$ be the set of sections $\Gamma_{K} \rightarrow \Pi_{X_{K}}$ of $\Pi_{X_{K}} \rightarrow \Gamma_{K}$, considered up to composition with an inner automorphism arising from $\Delta_{X_{K}}$. Then the natural map

$$
X_{K}(K) \rightarrow \operatorname{Sect}\left(\Gamma_{K}, \Pi_{X_{K}}\right)
$$

is injective.

Remark. The so-called "Section Conjecture" states that (for instance when $X_{K}$ is proper) the natural map in Theorem 19.1 is bijective. (When $X_{K}$ is affine, the statement must be modified slightly.) At the time of writing (July 1996), the Section Conjecture has not yet been proven.

Proof. (of Theorem 19.1) Let $H_{X}$ be the abelianization of $\Delta_{X}$. First, note that by replacing $X_{K}$ by some finite Galois étale covering of $X_{K}$ of $p$-power order, we may assume 
that the compactification $\bar{X}_{K}$ of $X_{K}$ is itself hyperbolic and non-hyperelliptic. Let $r$ be the number of points in $\left(\bar{X}_{K}-X_{K}\right)(\bar{K})$. Let $Z_{X}$ be the complement of the diagonal in $X_{K} \times_{K} X_{K}$. By taking the second projection $Z_{X} \rightarrow X_{K}$, we may regard $Z_{X}$ as a family (parametrized by $X_{K}$ ) of smooth hyperbolic curves obtained by removing $r+1$ distinct points from some compactification.

Let $\eta_{X}$ be the generic point of $X_{K}$. Let $\bar{\eta}_{X}$ be the spectrum of some algebraic closure of $K\left(\eta_{X}\right)$. Let $Z_{\bar{\eta}_{X}} \stackrel{\text { def }}{=} Z_{X} \times_{X} \bar{\eta}_{X}$. Then (since $Z_{X} \rightarrow X_{K}$ is a family of smooth hyperbolic curves obtained by removing precisely $r+1$ distinct points from some compactification) we obtain an exterior Galois representation

$$
\rho_{Z}: \Pi_{X_{K}}^{\mathrm{prf}} \rightarrow \operatorname{Out}\left(\Delta_{Z_{\bar{\eta}_{X}}}\right)
$$

Now I claim that $\rho_{Z}$ factors through $\Pi_{X_{K}}$. Indeed, to see this, note that if $H_{Z_{\bar{\eta}_{X}}}$ is the abelianization of $\Delta_{Z_{\bar{\eta}_{X}}}$, then since $\Delta_{Z_{\bar{\eta}_{X}}}$ is a pro- $p$ group, the kernel of

$$
\Xi: \operatorname{Out}\left(\Delta_{Z_{\bar{\eta}_{X}}}\right) \rightarrow \operatorname{Aut}\left(H_{Z_{\bar{\eta}_{X}}}\right)
$$

is itself a pro- $p$ group. Thus, it suffices to show that $\Xi \circ \rho_{Z}$ maps $\Delta_{X}^{\text {prf }}$ into a pro- $p$ subgroup of $\operatorname{Aut}\left(H_{Z_{\bar{\eta}_{X}}}\right)$. But now observe that one has a Galois-equivariant surjection $H_{Z_{\bar{\eta}_{X}}} \rightarrow H_{X}$ whose kernel is either 0 or $\mathbf{Z}_{p}(1)$ (depending on whether $r=0$ or $r>0$ ). Moreover, the actions of $\Delta_{X}^{\mathrm{prf}}$ on $H_{X}$ and $\mathbf{Z}_{p}(1)$ are trivial. Thus, it follows immediately that the $\Delta_{X}^{\text {prf }}$-action on $H_{Z_{\bar{\eta}_{X}}}$ is by unipotent matrices, so $\Xi \circ \rho_{Z}$ maps $\Delta_{X}^{\text {prf }}$ into a pro- $p$ subgroup of $\operatorname{Aut}\left(M_{Z}\right)$, as desired. This completes the proof of the claim.

Thus, we have a representation

$$
\rho_{Z}: \Pi_{X_{K}} \rightarrow \operatorname{Out}\left(\Delta_{Z_{\bar{\eta}_{X}}}\right)
$$

Now suppose that we have two points $\alpha, \beta \in X_{K}(K)$ that induce the same element $\phi \in$ $\operatorname{Sect}\left(\Gamma_{K}, \Pi_{X_{K}}\right)$. Let $Z_{\alpha}$ (respectively, $Z_{\beta}$ ) be the pull-back of $Z_{X} \rightarrow X$ via $\alpha$ (respectively, $\beta)$. Thus, $Z_{\alpha}$ and $Z_{\beta}$ are hyperbolic curves over $K$. Moreover, since the action of $\Gamma_{K}$ on $\Delta_{Z_{\alpha}}$ (respectively, $\Delta_{Z_{\beta}}$ ) is determined by composing $\phi$ with $\rho_{Z}$, it follows that there exists some isomorphism $\psi: \Delta_{Z_{\alpha}} \cong \Delta_{Z_{\beta}}$ such that (i) $\psi$ is compatible with the respective outer $\Gamma_{K}$-actions; (ii) $\psi$ preserves and induces the identity between the quotients $\Delta_{Z_{\alpha}} \rightarrow H_{X}$, $\Delta_{Z_{\beta}} \rightarrow H_{X}$. Thus, by Theorem 16.5 (in fact, really, all we need is Theorem $A$ of [Mzk2]), it follows that $\psi$ arises from some isomorphism $Z_{\alpha} \cong Z_{\beta}$ which is compatible with and induces the identity on the inclusions $Z_{\alpha} \hookrightarrow X_{K}, Z_{\beta} \hookrightarrow X_{K}$. But this clearly implies that $\alpha=\beta$, thus completing the proof of the Theorem.

Remark. Note that in fact, in the proof of Theorem 19.1, we really only used the weaker results of $[\mathrm{Mzk} 2]$ - i.e., we did not need to use Theorem $A$ of the present paper. On the 
other hand, at the time that [Mzk2] was written, the author was unaware of Theorem 19.1, which is the primary reason that Theorem 19.1 did not appear in [Mzk2]. Note also that although Theorem 19.1 implies a corresponding profinite result, the profinite result can be proven much more easily, by using the "Kummer exact sequence" for the Jacobian of $X_{K}$. Finally, it should be remarked that the argument employed in the proof of Theorem 19.1 (as well as the Kummer sequence argument just mentioned) have been well-known for some time (see, e.g., [Naka2]). The main reason that Theorem 19.1 was included in this paper was that the author just wanted to make explicit that a $p r o-p$ injectivity result (such as Theorem 19.1) could now be proven.

\section{APPENDIX: A Grothendieck Conjecture-Type Result for Certain Hyperbolic Surfaces}

\section{Section a0: Introduction}

In this Appendix, we show how Theorem $A$ (cf. the Introduction) can be used to prove a Grothendieck Conjecture-type result for certain types of surfaces. The surfaces considered are families of (smooth) hyperbolic curves that are parametrized by hyperbolic curves (cf. Definition a2.1). We call such surfaces hyperbolically fibred. Our notation is similar to that of the rest of the paper, except that since here we consider only profinite (i.e., not pro- $p$ ) fundamental groups, in this Appendix, we will write $\Pi_{X_{K}}$ for the profinite fundamental group of $X_{K}$ :

Notation: If $K$ is a field and $X_{K}$ is a $K$-scheme, we denote by $\Pi_{X_{K}} \stackrel{\text { def }}{=} \pi_{1}\left(X_{K}\right)$ the fundamental group of $X_{K}$ (for some choice of base-point), and by $\Gamma_{K}$ the absolute Galois group of $K$. Then we have a natural morphism $\Pi_{X_{K}} \rightarrow \Gamma_{K}$ whose kernel $\Delta_{X} \subseteq \Pi_{X_{K}}$ is the geometric fundamental group $\pi_{1}\left(X_{K} \otimes_{K} \bar{K}\right)$ (where $\bar{K}$ is an algebraic closure of $K$ ).

Our main theorem is the following:

Theorem D. Let $K$ be sub-p-adic (cf. Definition 15.4 (i)). Let $X_{K}$ and $Y_{K}$ be hyperbolically fibred surfaces over $K$. Let $\operatorname{Isom}_{K}\left(X_{K}, Y_{K}\right)$ be the set of $K$-isomorphisms (in the category of $K$-schemes) between $X_{K}$ and $Y_{K}$. Let $\operatorname{Isom}_{\Gamma_{K}}\left(\Pi_{X_{K}}, \Pi_{Y_{K}}\right)$ be the set of continuous group isomorphisms $\Pi_{X_{K}} \rightarrow \Pi_{Y_{K}}$ over $\Gamma_{K}$, considered up to composition with an inner automorphism arising from $\Delta_{Y}$. Then the natural map

$$
\operatorname{Isom}_{K}\left(X_{K}, Y_{K}\right) \rightarrow \operatorname{Isom}_{\Gamma_{K}}\left(\Pi_{X_{K}}, \Pi_{Y_{K}}\right)
$$


is bijective.

This Theorem is given as Theorem a2.4 in the text.

We remark that:

(1) Theorem $D$ above is (modulo a certain technical result - Lemma a1.1) essentially derived from Theorem $A$. Note that one needs the full power of the "Hom version" of the Grothendieck Conjecture for hyperbolic curves (i.e., Theorem $A$ ) in order to prove an "Isom-type result" for surfaces (i.e., Theorem $D$ above). That is to say, the "Isom version" of Theorem $A$ of the present paper - i.e., Theorem $A$ of [Mzk2] - is not sufficient to prove Theorem $D$ above. Moreover, even with the "Hom version" of the Grothendieck Conjecture for hyperbolic curves (i.e., Theorem $A$ ), at the present time, I am unable to prove a "Hom version" of Theorem $D$.

(2) One might ask whether Theorem $D$ can be extended to hyperbolically fibered varieties (i.e., varieties obtained as successive fibrations of hyperbolic curves) of higher dimension. The problem is that just as we needed (see Remark (1)) the "Hom version" in dimension one to prove an "Isom-type result" in dimension two, we would need a "Hom-type result" in dimension two - which is currently not available - in order to prove an "Isom-type result" in, say, dimension three. Thus, at the present time, we are unable to advance beyond dimension two.

(3) Since Theorem $A$ is valid in the pro- $p$ case as well, one might ask why one cannot prove a pro- $p$ version of Theorem $D$ above. The problem is that the process of passing to the maximal pro- $p$ quotient is not well-behaved with respect to fibrations. That is to say, if $X_{K} \rightarrow X_{K}^{\prime}$ is a family of hyperbolic curves parametrized by a hyperbolic curve (as in Definition a2.1), then the maximal pro- $p$ quotient of the fundamental group of the geometric generic fiber of $X_{K} \rightarrow X_{K}^{\prime}$ does not map injectively (in general) to the maximal pro- $p$ quotient of $\Delta_{X}$. Thus, any attempt to prove a pro- $p$ version of Theorem $D$ by means of the techniques employed here would result in a rather unnatural theorem.

(4) Since Theorem $A$ admits various truncated versions (cf. the Introduction) as well, one might ask why one cannot prove a truncated version of Theorem $D$. The problem here is the same as the problem that arises when one tries to prove a pro- $p$ result: i.e., truncating is not well-behaved with respect to fibrations.

(5) To a slight extent, the content of Theorem $D$ above overlaps with recent results of H. Nakamura and N. Takao (see Theorem $A$ and Corollary $B$ of $[\mathrm{NT}])$. 


\section{Section a1: A Key Lemma}

In this Section, we prove a simple technical lemma which will be the key technical ingredient that allows us to extend Theorem $A$ to the case of surfaces. Let $K$ be an algebraically closed field of characteristic zero. Let $X$ and $Y$ be hyperbolic curves over $K$ (cf. Section 0 for a definition of this term).

Lemma a1.1. Let $\phi: X \rightarrow Y$ be a finite $K$-morphism. Let $\psi: \Pi_{X_{K}} \rightarrow \Pi_{Y_{K}}$ be the induced morphism on fundamental groups. Suppose that $\operatorname{Ker}(\psi)$ is topologically finitely generated. Then $\phi$ is étale.

Proof. By replacing $Y$ by the finite étale covering of $Y$ corresponding to $\operatorname{Im}(\psi) \subseteq \Pi_{Y_{K}}$, we may assume that $\psi$ is surjective. Under this assumption, $\phi$ is étale if and only if $\phi$ is an isomorphism. Thus, it suffices to assume that:

(1) $\operatorname{Ker}(\phi)$ is topologically finitely generated; and

(2) $\phi$ is not an isomorphism (hence has degree $>1$ )

and derive a contradiction.

Let $Y^{\prime} \rightarrow Y$ be a finite étale covering $Y$, where $Y^{\prime}$ is connected. Let $X^{\prime} \stackrel{\text { def }}{=} X \times_{Y} Y^{\prime}$. Then $X^{\prime} \rightarrow X$ is finite étale, and it follows from the assumption that $\psi$ is surjective that $X^{\prime}$ is connected. Since $Y$ is hyperbolic, it follows that for a suitable choice of $Y^{\prime} \rightarrow Y, Y^{\prime}$ will have genus $\geq 2$. Thus, by replacing our original $X \rightarrow Y$ by $X^{\prime} \rightarrow Y^{\prime}$, we may assume that $X^{\prime}$ and $Y^{\prime}$ have genus $\geq 2$.

Since $X \rightarrow Y$ is assumed to have degree $>1$, it follows that $g_{X}$ (the genus of $X$ ) is strictly greater than $g_{Y}$ (the genus of $Y$ ). Let $Y^{\prime} \rightarrow Y$ be a finite étale covering of degree $d$ such that $Y^{\prime}$ is connected, and $Y^{\prime} \rightarrow Y$ extends to an étale covering over some compactification of $Y$. Then the Riemann-Hurwitz formula implies that, as $Y^{\prime} \rightarrow Y$ varies, $g_{Y^{\prime}}$ (which we think of as a function of $d$ ) is equal to $d\left(g_{Y}-1\right)+1$. On the other hand, if $X^{\prime} \stackrel{\text { def }}{=} X \times_{Y} Y^{\prime}$, then (again by the Riemann-Hurwitz formula) $g_{X^{\prime}}=d\left(g_{X}-1\right)+1$. Thus, it follows that as $d \rightarrow \infty$, the difference $g_{X^{\prime}}-g_{Y^{\prime}} \rightarrow \infty$.

Now let $H_{X^{\prime}}$ denote the first homology group of the compactification of $X^{\prime}$ with coefficients in $\mathbf{Z}_{p}$ (for some prime number $p$ which will be fixed throughout the discussion).

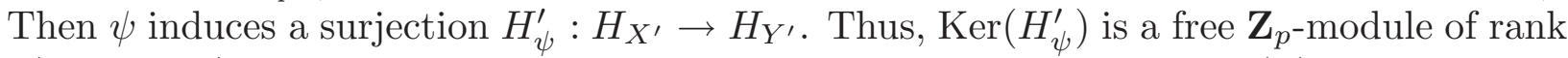
$2\left(g_{X^{\prime}}-g_{Y^{\prime}}\right)$. On the other hand, any topological generators of $\operatorname{Ker}(\psi)$ clearly define a set of $\mathbf{Z}_{p^{\prime}}$-generators of $\operatorname{Ker}\left(H_{\psi}^{\prime}\right)$. But this implies that $\operatorname{rank}_{\mathbf{Z}_{p}}\left(\operatorname{Ker}\left(H_{\psi}^{\prime}\right)\right)$ is bounded, independently of $Y^{\prime} \rightarrow Y$, which contradicts the fact that $\operatorname{rank}_{\mathbf{z}_{p}}\left(\operatorname{Ker}\left(H_{\psi}^{\prime}\right)\right)=2\left(g_{X^{\prime}}-\right.$ $\left.g_{Y^{\prime}}\right) \rightarrow \infty$ as $d \rightarrow \infty$. This contradiction completes the proof. 


\section{Section a2: The Main Theorem}

Let $K$ be a field of characteristic zero. Let $X_{K}$ be a surface over $K$, by which we mean that $X_{K}$ is a smooth (geometrically connected) variety over $K$ of dimension two.

Definition a2.1. We shall say that $X_{K}$ is a hyperbolically fibred surface if the following condition holds: There exists a hyperbolic curve $X_{K}^{\prime}$ over $K$, together with a smooth, proper, connected morphism $\bar{X}_{K} \rightarrow X_{K}^{\prime}$ of relative dimension one such that $X_{K}$ embeds as an open subvariety of $\bar{X}_{K}$ satisfying the following conditions: (i) $\bar{X}_{K}-X_{K}$ is a divisor in $\bar{X}_{K}$ which is étale over $X_{K}^{\prime}$; (ii) the geometric fibers of $X_{K} \subseteq \bar{X}_{K} \rightarrow X_{K}^{\prime}$ are hyperbolic curves.

If $X_{K}$ is a hyperbolically fibred surface, then we shall refer to $X_{K} \rightarrow X_{K}^{\prime}$ (as above) as a parametrizing morphism for $X_{K}$.

Note that given a hyperbolically fibered surface $X_{K}$, in general, there can exist more than one parametrizing morphism for $X_{K}$. If $X_{K} \rightarrow X_{K}^{\prime}$ is a parametrizing morphism for $X_{K}$, and $F_{\Omega} \subseteq X_{K}$ is a fiber of this morphism (over some point of $X_{K}(\Omega)$ valued in an algebraically closed field $\Omega$ ), then we have an exact sequence of fundamental groups:

$$
1 \rightarrow \Pi_{F_{\Omega}} \rightarrow \Pi_{X_{K}} \rightarrow \Pi_{X_{K}^{\prime}} \rightarrow 1
$$

Note that since $F_{\Omega}$ and $X_{K}^{\prime}$ are hyperbolic curves, it follows that $\Pi_{F_{\Omega}}$ and $\Pi_{X_{K}^{\prime}}$ (hence also $\left.\Pi_{X_{K}}\right)$ are topologically finitely generated.

Now let $K$ be sub-p-adic (cf. Definition 15.4 (i)) (for some prime number $p$ ), and let $X_{K}$ and $Y_{K}$ be hyperbolically fibred surfaces over $K$. Let

$$
\phi: \Pi_{X_{K}} \rightarrow \Pi_{Y_{K}}
$$

be a continuous group isomorphism over $\Gamma_{K}$. We would like to show that $\phi$ arises geometrically.

Let $\zeta_{Y}: Y_{K} \rightarrow Y_{K}^{\prime}$ be a parametrizing morphism for $Y_{K}$. Then by composing $\phi$ with $\pi_{1}\left(\zeta_{Y}\right)$, we obtain a continuous surjection $\Pi_{X_{K}} \rightarrow \Pi_{Y_{K}^{\prime}}$. By Theorem $A$, it follows that this surjection arises geometrically, from some morphism $X_{K} \rightarrow Y_{K}^{\prime}$. Thus, we may regard $X_{K}$ and $Y_{K}$ as objects over $Y_{K}^{\prime}$. Let $Z_{K}$ be the normalization of $Y_{K}^{\prime}$ in $X_{K}$. Thus, the morphism $X_{K} \rightarrow Y_{K}^{\prime}$ factors through $\delta: Z_{K} \rightarrow Y_{K}^{\prime}$. Observe that $Z_{K}$ is a smooth, geometrically connected (since $X_{K}$ is geometrically connected over $K$ ) curve over $K$, and that $Z_{K} \rightarrow Y_{K}^{\prime}$ is finite. (In particular, since $Y_{K}^{\prime}$ is hyperbolic, it follows that $Z_{K}$ is also hyperbolic.) Moreover, it follows from the definition of $Z_{K}$ that the morphism $\Pi_{X_{K}} \rightarrow$ $\Pi_{Z_{K}}$ induced by $X_{K} \rightarrow Z_{K}$ is surjective. This implies that $\operatorname{Ker}(\pi(\delta))$ is a quotient of $\operatorname{Ker}\left(\Pi_{X_{K}} \rightarrow \Pi_{Y_{K}^{\prime}}\right) \cong \operatorname{Ker}\left(\Pi_{Y_{K}} \rightarrow \Pi_{Y_{K}^{\prime}}\right)$, which (by the definition of a hyperbolically fibred 
surface) is topologically finitely generated. Thus, it follows that $\operatorname{Ker}(\pi(\delta))$ is topologically finitely generated. But then Lemma a1.1 implies that $Z_{K} \rightarrow Y_{K}^{\prime}$ is étale. On the other hand, since $\Pi_{X_{K}} \rightarrow \Pi_{Y_{K}^{\prime}}$ is surjective, we thus see that $Z_{K} \rightarrow Y_{K}^{\prime}$ must be an isomorphism. In particular, we thus conclude that the generic fiber of $X_{K} \rightarrow Y_{K}^{\prime}$ is smooth, geometrically connected, and of dimension one.

In fact, the argument of the preceding paragraph can be applied more generally to coverings of $X_{K}$, as follows: Let $\widetilde{Y}_{K}^{\prime} \rightarrow Y_{K}^{\prime}$ be a finite étale covering such that $\widetilde{Y}_{K}^{\prime}$ is geometrically connected over $K$. Let $\widetilde{Y}_{K} \rightarrow Y_{K} \times_{Y_{K}^{\prime}} \widetilde{Y}_{K}^{\prime}$ be a finite étale covering such that $\widetilde{Y}_{K} \rightarrow \widetilde{Y}_{K}^{\prime}$ is geometrically connected. Let $\widetilde{X}_{K} \rightarrow X_{K}$ be the covering corresponding (via $\phi)$ to $\tilde{Y}_{K} \rightarrow Y_{K}$. Thus, $\tilde{X}_{K} \rightarrow X_{K}$ factors through $\tilde{X}_{K} \rightarrow X_{K} \times_{Y_{K}^{\prime}} \tilde{Y}_{K}^{\prime}$. Then I claim that $\widetilde{X}_{K} \rightarrow \widetilde{Y}_{K}^{\prime}$ is geometrically connected. Indeed, this follows by the same argument as that employed in the preceding paragraph: Namely, we simply observe that $\Pi_{\widetilde{X}_{K}} \rightarrow \Pi_{\widetilde{Y}_{K}^{\prime}}$ is isomorphic to $\Pi_{\widetilde{Y}_{K}} \rightarrow \Pi_{\widetilde{Y}_{K}^{\prime}}$, which is surjective with topologically finitely generated kernel. This proves the claim. Let $L$ be the function field of $Y_{K}^{\prime}$; let $\bar{L}$ be its algebraic closure. Note that the natural map $\operatorname{Spec}(\bar{L}) \rightarrow Y_{K}^{\prime}$ factors through $\tilde{Y}_{K}^{\prime}$. Moreover, the above claim implies that if we base-change $\widetilde{X}_{K} \rightarrow \widetilde{Y}_{K}^{\prime}$ via $\operatorname{Spec}(\bar{L}) \rightarrow \widetilde{Y}_{K}^{\prime}$, the resulting $\widetilde{X}_{K} \times_{\widetilde{Y}_{K}^{\prime}} \operatorname{Spec}(\bar{L})$ is connected.

Now let us reinterpret the conclusions of the preceding paragraph in terms of fundamental groups. Let $\eta_{Y^{\prime}}$ be the generic point of $Y_{K}^{\prime}$. Let $Y_{\eta_{Y^{\prime}}} \stackrel{\text { def }}{=} Y_{K} \times_{Y_{K}^{\prime}} \eta_{Y^{\prime}} ; X_{\eta_{Y^{\prime}}} \stackrel{\text { def }}{=}$ $X_{K} \times_{Y_{K}^{\prime}} \eta_{Y^{\prime}}$. Then what we did in the preceding paragraph implies precisely that the morphism

$$
\operatorname{Ker}\left(\pi_{1}\left(X_{\eta_{Y^{\prime}}}\right) \rightarrow \pi_{1}\left(\eta_{Y^{\prime}}\right)\right) \rightarrow \operatorname{Ker}\left(\pi_{1}\left(Y_{\eta_{Y^{\prime}}}\right) \rightarrow \pi_{1}\left(\eta_{Y^{\prime}}\right)\right)=\operatorname{Ker}\left(\Pi_{Y_{K}} \rightarrow \Pi_{Y_{K}^{\prime}}\right)
$$

(induced by $\phi$ ) is surjective. Thus, we see that by composing the natural surjection of $\pi_{1}\left(X_{\eta_{Y^{\prime}}}\right)$ onto $\Pi_{X_{K}}$ with $\phi$, we obtain a continuous surjective group homomorphism

$$
\phi_{\eta_{Y^{\prime}}}: \Pi_{X_{\eta_{Y^{\prime}}}} \rightarrow \Pi_{Y_{\eta_{Y^{\prime}}}}
$$

over $\Gamma_{\eta_{Y^{\prime}}}$ (where we regard $X_{\eta_{Y^{\prime}}}$ and $Y_{\eta_{Y^{\prime}}}$ as curves over $\eta_{Y^{\prime}}$ ). Now we would like to apply Theorem $A$ again. This Theorem tells us that $\phi_{\eta_{Y^{\prime}}}$ arises geometrically from some $\eta_{Y^{\prime}-\text { morphism }} X_{\eta_{Y^{\prime}}} \rightarrow Y_{\eta_{Y^{\prime}}}$.

Remark. Note that in the argument of the preceding three paragraphs, it was absolutely essential to invoke Lemma a1.1 before we could apply Theorem $A$. In fact, if the variety "on the left" (i.e., in this case, $X_{\eta_{Y^{\prime}}}$ ) is not geometrically connected over the base field (i.e., in this case, $\bar{L}$ ), then it is not difficult to see that Theorem $A$ does not hold. Indeed, to construct such a counterexample, suppose that $Y_{K}$ is proper, and let $H_{K} \subseteq Y_{K}$ be a 
hyperplane section (with respect to some projective embedding of $Y_{K}$ ) such that $H_{K}$ is smooth and geometrically connected over $K$, and $\pi_{1}\left(H_{K}\right) \rightarrow \pi_{1}\left(Y_{K}\right)$ is surjective. (Such an $H_{K}$ exists by the Lefshetz hyperplane theorem.) Write $\eta_{H}$ for the generic point of $H_{K}$, and take for our " $X_{\eta_{Y^{\prime}}}$ " any hyperbolic curve over $\eta_{H}$. Then note that (if $H_{K}$ is sufficiently generic so that every fiber of $H_{K} \rightarrow Y_{K}^{\prime}$ contains at least one point at which $H_{K} \rightarrow Y_{K}^{\prime}$ is étale, then) the induced morphism $\pi_{1}\left(\eta_{H}\right) \rightarrow \pi_{1}\left(Y_{\eta_{Y^{\prime}}}\right)$ is also surjective, so we get a surjective morphism $\pi_{1}\left(X_{\eta_{Y^{\prime}}}\right) \rightarrow \pi_{1}\left(Y_{\eta_{Y^{\prime}}}\right)$ (by composing the (necessarily surjective) structure morphism $\pi_{1}\left(X_{\eta_{Y^{\prime}}}\right) \rightarrow \pi_{1}\left(\eta_{H}\right)$ with $\left.\pi_{1}\left(\eta_{H}\right) \rightarrow \pi_{1}\left(Y_{\eta_{Y^{\prime}}}\right)\right)$. But this surjective morphism does not arise from a dominant morphism $X_{\eta_{Y^{\prime}}} \rightarrow Y_{\eta_{Y^{\prime}}}$.

This completes the portion of the proof which is an application of (the nontrivial, surjectivity part of) Theorem $A$. The remainder of the proof will consists of using elementary algebraic geometry to show that the morphism $X_{\eta_{Y^{\prime}}} \rightarrow Y_{\eta_{Y^{\prime}}}$ constructed above extends to a morphism $X_{K} \rightarrow Y_{K}$.

Lemma a2.2. The morphism $X_{\eta_{Y^{\prime}}} \rightarrow Y_{\eta_{Y^{\prime}}}$ above extends to a morphism $X_{K} \rightarrow Y_{K}$.

Proof. First, observe that there exists a finite étale covering $V_{K} \rightarrow Y_{K}$ with the following properties:

(1) $V_{K}$ is a hyperbolically fibered surface that admits a parametrizing morphism $V_{K} \rightarrow V_{K}^{\prime}$ that fits into a commutative diagram:

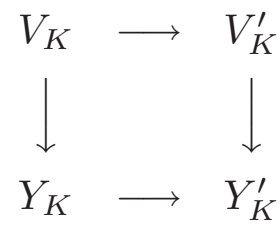

(2) The fibers of $V_{K} \rightarrow V_{K}^{\prime}$ have genus $\geq 2$.

Let $U_{K} \rightarrow X_{K}$ be the finite étale covering that corresponds (via $\phi$ ) to $V_{K} \rightarrow Y_{K}$. Let $\bar{V}_{K} \rightarrow V_{K}^{\prime}$ be the family of proper hyperbolic curves that compactifies $V_{K} \rightarrow V_{K}^{\prime}$ (as in Definition a2.1). Write $\eta_{V^{\prime}}$ for the generic point of $V_{K}^{\prime}$. Note that there exists a natural map $U_{K} \rightarrow V_{K}^{\prime}$ covering $X_{K} \rightarrow Y_{K}^{\prime}$, and that both $U_{K}$ and $V_{K}$ are geometrically connected over $\eta_{V^{\prime}}$ (cf. the argument used above to show that $\tilde{X}_{K} \rightarrow \tilde{Y}_{K}^{\prime}$ is geometrically connected). Thus, we may form (geometrically connected) $\eta_{V^{\prime}}$-curves $U_{\eta_{V^{\prime}}}, V_{\eta_{V^{\prime}}}$. Moreover, by the definition of $U_{K}$, we have a commutative diagram

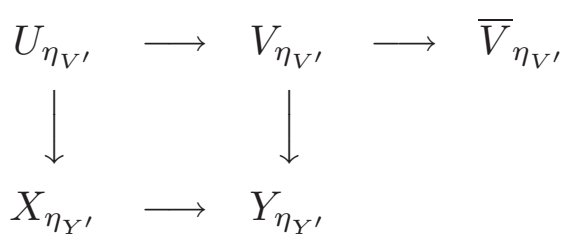


Thus, in particular, we obtain a morphism $U_{\eta_{V^{\prime}}} \rightarrow \bar{V}_{\eta_{V^{\prime}}}$ (over $\eta_{V^{\prime}}$ ). I claim that this morphism extends to a morphism $U_{K} \rightarrow \bar{V}_{K}$. Indeed, by the elementary theory of surfaces - "elimination of indeterminacy" (see, e.g., Theorem 5.5 of Chapter V of [Harts]) - it follows that there exists some $\widetilde{U}_{K} \rightarrow U_{K}$ (obtained by successively blowing up smooth points) such that the birational transformation from $U_{K}$ to $\bar{V}_{K}$ defined by $U_{\eta_{V^{\prime}}} \rightarrow \bar{V}_{\eta_{V^{\prime}}}$ extends to a morphism $\widetilde{U}_{K} \rightarrow \bar{V}_{K}$. On the other hand, any exceptional curve in $\widetilde{U}_{K}$ necessarily maps into a fiber of $\bar{V}_{K} \rightarrow V_{K}^{\prime}$ (this follows since $U_{K} \rightarrow V_{K}^{\prime}$ is already a morphism). Thus, any exceptional $\mathbf{P}^{1}$ in $\widetilde{U}_{K}$ maps quasi-finitely into a fiber of $\bar{V}_{K} \rightarrow V_{K}^{\prime}$ (which will always be a smooth, proper, hyperbolic curve) - which is clearly absurd. This contradiction completes the proof of the claim.

Thus, we have a morphism $U_{K} \rightarrow \bar{V}_{K}$. Let $\bar{Y}_{K} \rightarrow Y_{K}^{\prime}$ compactify $Y_{K} \rightarrow Y_{K}^{\prime}$ (as in Definition a2.1). Then clearly $V_{K} \rightarrow Y_{K}$ extends to a finite (in general, ramified) morphism $\bar{V}_{K} \rightarrow \bar{Y}_{K}$. Thus, to summarize, we have a rational map from $X_{K}$ to $\bar{Y}_{K}$ which is covered by a morphism $U_{K} \rightarrow \bar{V}_{K}$, where $U_{K}$ (respectively, $\bar{V}_{K}$ ) is finite over $X_{K}$ (respectively, $\left.\bar{Y}_{K}\right)$. By elementary algebraic geometry, this implies that we get a morphism $X_{K} \rightarrow \bar{Y}_{K}$ (covered by $U_{K} \rightarrow \bar{V}_{K}$ ).

To complete the proof of the Lemma, we must verify that this morphism $X_{K} \rightarrow \bar{Y}_{K}$ factors through $Y_{K}$. To do this, we simply choose $V_{K} \rightarrow Y_{K}$ in the above argument such that $V_{K}$ is ramified, with very large (say, compared to the degree of $X_{K} \rightarrow \bar{Y}_{K}$ ) ramification index, over all of the divisor $\bar{Y}_{K}-Y_{K}$. (Note that such coverings exist, by the exact sequence of fundamental groups following Definition a2.1, plus the well-known fact from topology that the fundamental group of a compact surface with finitely many punctures has coverings with arbitrarily large ramification index at those punctures.) Then since $X_{K} \rightarrow \bar{Y}_{K}$ is covered by $U_{K} \rightarrow \bar{V}_{K}$, and $U_{K} \rightarrow X_{K}$ is finite étale, we see that we obtain a contradiction, unless $X_{K} \rightarrow \bar{Y}_{K}$ maps into $Y_{K}$. Thus, we conclude that we have a morphism $X_{K} \rightarrow Y_{K}$, as desired.

Let us summarize what we have done so far. We started with a continuous group isomorphism

$$
\phi: \Pi_{X_{K}} \rightarrow \Pi_{Y_{K}}
$$

over $\Gamma_{K}$. From $\phi$, we constructed a $K$-morphism $X_{K} \rightarrow Y_{K}$, which we denote by $\alpha$. Since $\phi$ is invertible, we thus see that we have also constructed a $K$-morphism $\beta: Y_{K} \rightarrow X_{K}$ from $\phi^{-1}$. Since everything we have been doing is functorial, it follows that $\pi_{1}(\beta \circ \alpha)$ is the identity on $\Pi_{X_{K}}$ (up to composition with an inner automorphism arising from an element of $\Delta_{X}$ ). Thus, by Lemma a2.3 below, we conclude that $\beta \circ \alpha$ is the identity on $X_{K}$. Similarly, $\alpha \circ \beta$ is the identity on $Y_{K}$.

Lemma a2.3. Let $\gamma: X_{K} \rightarrow X_{K}$ be a K-morphism such that $\pi_{1}(\gamma)$ is the identity on $\Pi_{X_{K}}$ (up to composition with an inner automorphism arising from an element of $\Delta_{X}$ ). Then $\gamma$ is the identity. 
Proof. First observe that $\pi_{1}(\gamma)$ is trivially compatible (up to composition with a geometric inner automorphism) with the map induced by $\pi_{1}$ 's by $X_{K} \rightarrow X_{K}^{\prime}$. Thus, (the injectivity part of) Theorem $A$ tells us that $\gamma$ is compatible with $X_{K} \rightarrow X_{K}^{\prime}$. Let $\eta_{X^{\prime}}$ be the generic point of $X_{K}^{\prime}$. Then $\gamma$ defines a morphism $\gamma_{\eta_{X^{\prime}}}: X_{\eta_{X^{\prime}}} \rightarrow X_{\eta_{X^{\prime}}}$ which induces the identity (up to composition with a geometric inner automorphism) on $\pi_{1}$ 's. Applying (the injectivity part of) Theorem $A$ again then tells us that $\gamma_{\eta_{X^{\prime}}}$ is the identity. Thus, it follows that $\gamma$ is the identity, as desired.

Thus, $\alpha$ and $\beta$ are isomorphisms. Moreover, it follows from the construction of $\alpha$ that $\pi_{1}(\alpha)$ coincides with $\phi$ (up to composition with an inner automorphism arising from an element of $\Delta_{Y}$ ), and it follows from Lemma a2.3 that $\alpha$ is the unique such $K$-isomorphism $X_{K} \cong Y_{K}$. Thus, we see that we see that we have proven the following result:

Theorem a2.4. Let $K$ be sub-p-adic (cf. Definition 15.4 (i)). Let $X_{K}$ and $Y_{K}$ be hyperbolically fibred surfaces over $K$. Let $\operatorname{Isom}_{K}\left(X_{K}, Y_{K}\right)$ be the set of $K$-isomorphisms (in the category of $K$-schemes) between $X_{K}$ and $Y_{K}$. Let $\operatorname{Isom}_{\Gamma_{K}}\left(\Pi_{X_{K}}, \Pi_{Y_{K}}\right.$ ) be the set of continuous group isomorphisms $\Pi_{X_{K}} \rightarrow \Pi_{Y_{K}}$ over $\Gamma_{K}$, considered up to composition with an inner automorphism arising from $\Delta_{Y}$. Then the natural map

$$
\operatorname{Isom}_{K}\left(X_{K}, Y_{K}\right) \rightarrow \operatorname{Isom}_{\Gamma_{K}}\left(\Pi_{X_{K}}, \Pi_{Y_{K}}\right)
$$

is bijective.

\section{Bibliography}

[AV] D. Mumford, Abelian Varieties, Oxford Univ. Press (1974).

[BK] S. Bloch and K. Kato, L-Functions and Tamagawa Numbers in The Grothendieck Festschrift, Volume I, Birkhäuser (1990), pp. 333-400.

[Del] P. Deligne, Le Groupe Fondamental de la Droite Projective Moins Trois Points in Galois Groups over Q ed. by Y. Ihara, K. Ribet, J.-P. Serre, Springer Verlag (1989), pp. 79-297.

[DM] P. Deligne and D. Mumford, The Irreducibility of the Moduli Space of Curves of Given Genus, IHES Publ. Math. 36 (1969), pp. 75-109.

[Falt1] G. Faltings, p-adic Hodge Theory, Journal of the Amer. Math. Soc. 1, No. 1 (1988), pp. 255-299.

[Falt2] G. Faltings, Crystalline Cohomology and p-adic Galois Representations, Proceedings of the First JAMI Conference, Johns-Hopkins University Press (1990), pp. 25-79. 
[Falt3] G. Faltings, Endlichkeitssätze für Abelschen Varietäten über Zahlkörpern, Inv. Math. 73 (1983), pp. 349-366.

[FC] G. Faltings and C.-L. Chai, Degenerations of Abelian Varieties, Springer Verlag (1990).

[Fo] J. M. Fontaine, Formes differentielles et modules de Tate des variétés abéliennes sur les corps locaux, Inv. Math. 65 (1982), pp. 379-409.

[Groth] A. Grothendieck, letter to G. Faltings (June 1983) in Lochak, L. Schneps, Geometric Galois Actions; 1. Around Grothendieck's Esquisse d'un Programme, London Math. Soc. Lect. Note Ser. 242, Cambridge Univ. Press (1997).

[Harts] R. Hartshorne, Algebraic Geometry, Graduate Texts in Mathematics 52, Springer Verlag (1977).

[Hiro] H. Hironaka, Resolution of Singularities of an Algebraic Variety over a field of Characteristic Zero, Ann. Math. 79 (1964), pp. 109-203; 205-326.

[Hyodo] O. Hyodo, On variation of Hodge-Tate structures, Math. Ann. 284 (1989), pp. 7-22.

[IN] Y. Ihara and H. Nakamura, Some illustrative examples for anabelian geometry in high dimensions in Lochak, L. Schneps, Geometric Galois Actions; 1. Around Grothendieck's Esquisse d'un Programme, London Math. Soc. Lect. Note Ser. 242, Cambridge Univ. Press (1997), pp. 127-138.

[Jou] J.-P. Jouanolou, Théorèmes de Bertini et Applications, Progress in Mathematics 42, Birkhäuser (1983).

[Kato] K. Kato, Logarithmic Structures of Fontaine-Illusie, Proceedings of the First JAMI Conference, Johns-Hopkins University Press (1990), pp. 191-224.

[Knud] F. F. Knudsen, The Projectivity of the Moduli Space of Stable Curves, II, Math. Scand. 52 (1983), pp. 161-199.

[Kobl] N. Koblitz, p-adic Numbers, p-adic Analysis, and Zeta Functions, Graduate Texts in Mathematics 58, Springer Verlag (1977).

[Lang] S. Lang, Abelian Varieties, Springer Verlag (1983).

[Mats] H. Matsumura, Commutative Algebra (Second Edition), The Benjamin/Cummings Publishing Company (1980).

[MB] L. Moret-Bailly, Exposé II in Séminaire sur les Pinceaux Arithmétiques: La Conjecture de Mordell, edited by L. Szpiro, Astérisque 127 (1985).

[Mzk1] S. Mochizuki, The Profinite Grothendieck Conjecture for Closed Hyperbolic Curves over Number Fields, J. Math. Sci., Univ. Tokyo 3 (1996), pp. 571-627.

[Mzk2] S. Mochizuki, The Local Pro-p Grothendieck Conjecture for Hyperbolic Curves, RIMS, Kyoto University, Preprint 1045 (1995).

[Mzk3] S. Mochizuki, The Geometry of the Compactification of the Hurwitz Scheme, Publ. of RIMS 31, No. 3 (1995), pp. 355-441. 
[Naka1] H. Nakamura, Galois Rigidity of the Étale Fundamental Groups of Punctured Projective Line, J. reine angew. Math. 411 (1990), pp. 205-216.

[Naka2] H. Nakamura, Galois Rigidity of Algebraic Mappings into some Hyperbolic Varieties, Intern. J. Math. 4 (1993), pp. 421-438.

[Naka3] H. Nakamura, On the Exterior Galois Representations Associated with Open Elliptic Curves, J. Math. Sci., Univ. Tokyo 2 (1995), pp. 197-231.

[NT] H. Nakamura and N. Takao, Galois Rigidity of pro-l Pure Braid Groups of Algebraic Curves, Trans. Amer. Math. Soc. 350 (1998), pp. 1079-1102.

[NTs] H. Nakamura and H. Tsunogai, Some finiteness theorems on Galois centralizers in pro-l mapping class groups, J. reine angew. Math. 441 (1993), pp. 115-144.

[Pop1] F. Pop, Grothendieck's Conjecture of Birational Anabelian Geometry, Ann. of Math. 138 (1994), pp. 145-182.

[Pop2] F. Pop, Grothendieck's Conjecture of Birational Anabelian Geometry II, Preprint Series Arithmetik II, No. 16, Heidelberg (1995).

[Pop3] F. Pop, oral communication to the author (July-August 1996).

[Ser1] J.-P. Serre, Groupes Algébriques et Corps de Classes, Hermann, Paris (1959).

[Ser2] J.-P. Serre, Local Fields, Graduate Texts in Mathematics 67, Springer Verlag (1979).

[SGA2] A. Grothendieck et al., Cohomologie Locale des Faisceaux Cohérents et Théorèmes de Lefshetz Locaux et Globaux, North-Holland, Amsterdam (1968).

[SGA4] A. Grothendieck et al., Théorie des Topos et Cohomologie Étale, Lecture Notes in Mathematics 264, 270, 305, Springer Verlag (1972-3).

[Sha] S. S. Shatz, Profinite Groups, Arithmetic, and Geometry, Annals of Math. Studies 67, Princeton University Press (1972).

[Shiho] A. Shiho, manuscript in preparation.

[Tama] A. Tamagawa, The Grothendieck Conjecture for Affine Curves, Compositio Math. 109, No. 2 (1997), pp. 135-194.

[Tate] J. Tate, p-Divisible Groups, Proceedings of a Conference on Local Fields, Driebergen, Springer Verlag (1967), pp. 158-183. 